University of Warwick institutional repository: http://go.warwick.ac.uk/wrap

A Thesis Submitted for the Degree of PhD at the University of Warwick

http://go.warwick.ac.uk/wrap/36682

This thesis is made available online and is protected by original copyright.

Please scroll down to view the document itself.

Please refer to the repository record for this item for information to help you to cite it. Our policy information is available from the repository home page. 
On the Theory of Fitting Clesses of Tinite Soluble Groups

F.P. Lockett

Thesis submitted for the Degree of Doctor of Philosophy at the University of Warvick 


\section{BEST COPY}

AVAILABLE

Poor text in the original thesis. 
Ve continue the study of Fitting clases begun by Fischer ir 1966 and carried on by (notably) Gaschütz end Hartloy. Disappointingly the theory has, as yet, failed to display the richness of its predecessor, the theory of Formations. Here ve present oun contributions, embedded in a survey of the procress so far made in this tantalizing part of finite soluble group theory.

Chapter 0 indicates the group thooretic notation we use, while Chapter 1 contains the basic results and terminology of Fitting class theory. Proddly speaking this theory comprises a study of the classes themrelves, a study of the embedding of the $\mathcal{F}$-subgroups (subgroups which belong to $\mathcal{F}$ ) of an erbitrary group $G$ and a study of the relation between $\mathcal{F}$ and the $\mathcal{F}$ subgroups of $G$. As in Formation theory ve focus attention on canonical sets of $\mathcal{F}$-suberoups, namely the $\mathcal{F}$-injectors, the Fischer $\mathcal{F}$-subgroups and the maximil $\mathcal{F}$-suberoups containing the radical.

Chapter 2 begins with analyses of several examples of Fitting classes, establishing the coincidence of these three sets of 7 subgroups (in all groups) in many cases, a. property not enjoyed by all Fitting classes. Here too we examine some known 'new classes from old' procedures, and introduce a new one $\mathcal{F}^{\pi}$ (defined for any $\mathcal{F}$ and set of primos $\pi$ ), showing how this concept may be used to characterize the injectors for the product $\mathcal{F}_{1} \mathcal{F}_{2}$ of two Fitting classes. The chapter ends with some remarks on the thorny problem of generating Fitting ciasses from given groups and we present an imitation of work of Dark, the only person to achieve progress in this direction. Finally we show how one of 
the classes so constructed settles a question posed by Gasichntr.

Chapter 3 develops the theory of pronomel. subrroups, besed on.key thcorems of Iann-Alperin and Fjscher, whoring in particular that a pernutable product of pronormal subrrouns is akain pronormal. This approach yields a more compact version of work on subcroups of a group which are p-nomally embedded for all primes $p$ (we use the term strongly pronomel), published by Chambers.' The injectors for a Fischer class (in particular a subgroup closed Fitting class) have this property.

Chapter 4 attacks the problem of determining the injectors for the class $\mathrm{f}^{\pi}$, and shows, in the light of chapter 3 , that the nature.l guess (a product of an $\mathcal{F}$-injector and a Hall $\pi^{\prime}$ subgroup) holds good when, for instance, $\mathcal{F}$ is a Fischer class. However, modification of the example of Dark denies that this is in general the case. So arises the concept of permutability of a Fitting class and, after giving a nev proof of a reloted lomma of Fischer, we establish conditions on a Fitting class equivalent to its being permutable, involving system normalizers.

Chapter 5 takes a preliminary look at the analogue of Cline's partial ordering of strong containment («) for Fitting classes, and we show that a Fitting class maximal in this sense and having strongly pronomal injectors in all groups, is necessarily a normal Fjtting class. In our final section we examine the radical of a direct power of a group $G$ rnd show that, for a normal class, the radical is never the corresponding direct pover of the radical of $G$ (unless of course $G$ lies in the class). This investigation puts the set of normal Fitting c.lasses in a new sotting, and we demonstrate that to each Fitting class $f$ there corresponds a well defined class $\mathcal{F}^{*}$ with properties close to those of $f$. 
I wish to thank my supervisor, Dr. 2.0. Hewkes of the Unjversity of Warwicl, for the inspiration, advice and encouragement which he has given me during my three vears of study at larwick. Further encouregement came from Professor J.C. Beid] oman of the University of Kentucky during the year he spont visiting varwick, to whom my thanks are also due. I am grateful to Professor R.H. Carter and Dr. B. Hartley for helpful conversations, and to my colleagues C.J. Fraddon and D.I. NacIean for their stimulating company.

I am indebted to the Science Research Council for a three year studentship, and to the Warwick University supervision system for the supplementary financial support it has provided.

To the best of my knowledge results presented here, and not attributed to others or described as well-known, are oxigina?. 
Chapter () Notiation and assumed results

Chopter 1 Introduction and preliminary rosults

1.1 Fitting clesses and $\mathcal{F}$-injectors 4

1.2 Fjscher classes and the sets $\operatorname{II}_{\mathcal{F}}(G) \quad 10$ and $\operatorname{III}_{\boldsymbol{f}}(G)$

Chapter 2 Examples of Fitting clesses

2.1 The nilpotent injectors 18

2.2 A class due to Gaschütz 21

2.3 Groups with centrel socle 25

2.4 loormal Fitting classes 31

2.5 Fitting formations 32

2.6 Ihe classes $\mathcal{F}^{\pi}, \mathcal{F}_{1} \mathcal{F}_{2}$ and $\mathcal{F}_{1} \cap \mathcal{F}_{2}$

2.7 Generating Pitting classes 45

$2.8 \wedge$ problem of Geschitz 51

Chapter 3 Pronormal and strongly pronormal subgroups

3.1 Fronormal subgroups 55

3.2 Strongly pronormal subgroups 60

3.3 Fitting classes with strongly pronormal 63 injectors

Chapter 4 Fermutability

4.1 Permutable Fitting classes 70

4.2 Fischer's lemma and conditions equivalent 76 to permutability

Chapter 5 Strong containment, normal Fitting classes and $\mathfrak{f}^{*}$

5.1 Strong containment 82

5.2 Normal Fitting clesses and $\mathcal{F}^{*} \quad 84$ 
- $\mathrm{I} \leqslant \mathrm{G}, \mathrm{H}<\mathrm{G}, \mathrm{H} \triangleleft \mathrm{G}$ menn that $\mathrm{H}$ is $\Rightarrow$ subsroup, propex subroup, nornel subgroup of G respectively; while $\mathrm{X} \subseteq \mathrm{Y}$, $X \subset Y$ indicate that $X$ is a subsot, propor subset of $Y$ rospectivcly. Apart fron this, our notation and terminology is that of Gorenstein's book, supnlemented $2 y$ the following, we hope well accopted, notation.

$\mathcal{X}$ is called a class of groups if $\mathcal{X}$ contains eveh Eroup of order 1 , and any exoup isomorphic to one of its members. An $\mathcal{X}$-group is then a member of $\mathcal{X}$. Unless otherwise stated, cach group considered in this dissortation will be finite and belong to $S$, the class of soluble croups. $O$ denotes the class of Abclian croups and $\mathcal{X}$ the class of nilpotent groups. $S_{\pi}$ is the class of soluble $\pi$-groups (where $\pi$ is a set of primes), and for any class $\mathfrak{X}, \mathfrak{X}_{\pi}$ is $\mathcal{X} \cap \mathcal{S}_{\pi}$. Frequent use will be made of the now familiar closure operation notation of Hall. A closure operation is an expanding, idempotent and monotonic function which raps classes of groups to classes of groups. For example s $\mathcal{X}$ is the class of all subrroups of $\mathcal{X}$-croups, and $Q \mathcal{X}$ the class of all quotients of $\mathcal{X}$-groups.

Ve assume knowledge of Hell's fundanerial theorems on the existence and conjugacy of Hall subgroups in finite soluble groups, and also his work on Sylow systems and system normalizers. $G_{p}$ will alvays denote a member of $\operatorname{SyI}_{p}(G)$, the set of Sylow p-subgroups of $G$, and $G_{\pi}$ a member of $\mathrm{HaII}_{\pi}(G)$, the set of Hall $\pi$-subgroups of $G$. We say $G$ reduces into the subgroup $H$ of $G$, written $G_{\pi} \searrow H$, if $G_{\pi} \cap H \in H_{2} I_{\pi}(H)$. $\Lambda$ sylow system $\Sigma$ of $G$ reduces into $H$, written $\Sigma>H$, if $G_{\pi}>H$ for all $G_{\pi} \in \Sigma$. $|G|_{\pi}$ denotes the greatest $\pi$-divisor of $|G|$, so $|G|_{\pi}=\left|G_{\pi}\right|$. 
$O_{\pi}(G)$ is the unirue lamest nom $\pi$-subiroun of $G$ and $G / 0^{\pi}(a)$ its unique lereost $\pi$-quotient.

To also aswne the reader is frmilion with the bosic projertios of Corter subroups and the elcnentis of Irormation theory. For those and twe other rosults ve need, Hupport's book is the nature r roferonce.

$G$ (soluble) is called primitive iff $G$ has a unicue mininal normal subrroup which is complemented iff $\mathrm{G}$ has a selfcentralizing miniral normal subgroup.

If $A, B \leqslant G ; A \perp B$ vill meen that $A$ and $B$ permute, that is $A B=B A$ or equivalently that $A B$ is a suberoup of $G$. If $A \triangleleft B \leqslant G$ and $I \leqslant G$, we soy II covers $\Lambda / B$ if $B=A(H \cap B)$ and avoids $A / 3$ if $I \cap B \leqslant A$. $\prod_{i \in I} G_{i}$ vill denote the direct product of the groups $\left\{\hat{v}_{i}\right\}_{i \in I} \cdot c_{t}$ is our notation for the cyclic group of order $t, \Sigma_{n}$ the symmetric group of degree $n, A_{n}$ its alternating subgroup, $D_{2 n}$ the dihedrsi group of order $2 n$ and $Q_{8}$ the quaternion Eroup. A $2 B$, the wreath product of $A$ by $B$, will be constructed from the regular permutation representation of $B$ unless another representation is specified. A conjugacy class $\mathrm{H}^{\mathrm{G}}$ of subgroups of $\mathrm{G}$ will be called a charecteristic conjugacy cless if each automorphism of $G$ permutes the members of $H^{G}$. $H$ is a subnormel subgroup of $G$, written $H$ sn $G$, if there exists a series $\mathrm{H} \triangleleft \mathrm{H}_{1} \triangleleft \mathrm{II}_{2} \triangleleft \ldots . \triangleleft \mathrm{H}_{n-1} \triangleleft \mathrm{G}$. H is a pronormal subcroup of $G$, vritton $H$ pro $G$, if $H$ and $H^{g}$ are conjugate in their join $\left\langle\mathrm{H}, \mathrm{H}^{\mathrm{E}}\right\rangle$ for all $\xi \in \mathrm{G}$. Soc G denotes the socle of $G$, the product of all the minimal normal subgroups of $G$; and $\pi-\operatorname{soc} G$, the $\pi$-socle of $G$, the product of all the minimal noimal $\pi$-suberoups of $G$. 


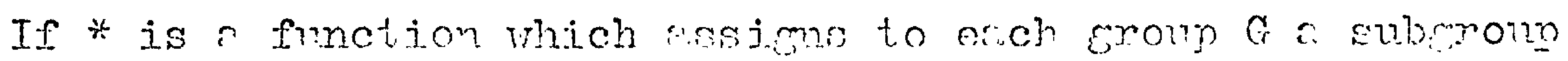
(respectitroly set of subsronss) *(C), then if $\mathrm{J} r G$,

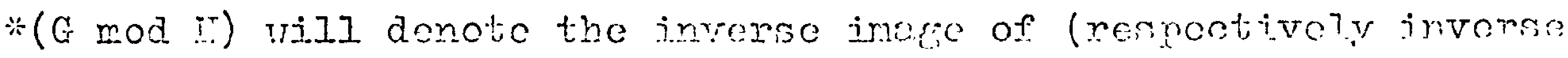
inciges of the mombers of $*(G / T)$ in $G$.

Groups and sets of operetors (including motrices) will cIroys act on the right.

We rrite $G=[H]$ thenever in comploments the norma] wuberoup $H$ of $G$, thet is, $G$ is a split extension of $I$ by $F$. The following key corollary of the Schur-zissenheus thesrem vill be rell used.

If $G=[H] T$ where $|H|$ and $|H|$ ere coprine then

a) $\bar{H}=[\mathrm{H}, \mathrm{I}] \mathrm{C}_{\mathrm{H}}(\mathrm{K})$ and the product is direct when $\mathrm{H} \in O$,

b) $[H, I]=[H, K, Y]$, so $[E, K, Y, \ldots, K]=1$

eventually (that is rith sufficient terms $I_{i}$ ) $\Rightarrow[H, K]=1$.

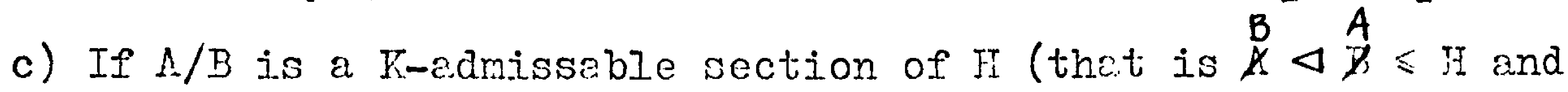
$K$ nomrizes $A$ and $B)$ then $C_{\Lambda / B}(K)=C_{A}(K) B / B$.

The proof requires the solubility of either $H$ or $K$, which holds in this dissertetion by the assumed solubility of each $G$, but is true in any case by the theorem of fieit and Thompson.

Frequent appeals will be made to the fact thet the Fitting: subrroup: $F(G)$ of a finite soluble group contcins its centralizer in $G$; and also to the vell-knom theorems of Iischlre and Clifford. 


\subsection{Fitting Classes and $\mathcal{F}$-injectors}

The concept of a Fitting class was introduced by Fischer in [1] (the main results of which appeer in [2]), to dualize Gaschütz's elegant theory of Formations. The name is a memorial to Hans Fitting, whose celebrated theorem [3] shows that the class of nilpotent groups is a Fitting class. A Fitting class $\mathcal{F}$ is a clasis of groups closcd under the operations of taking nomal subgroups and formins normol products, that is

$$
\begin{array}{ll}
N \triangleleft G \in \mathcal{F} \Rightarrow N \in \mathcal{F} & \left(\mathcal{F}=s_{n} \mathcal{F}\right) \\
\mathcal{F} \ni N_{1}, N_{2} \triangleleft G=N_{1} N_{2} \Rightarrow G \in \mathcal{F} & \left(\mathcal{F}=N_{0} \mathcal{F}\right)
\end{array}
$$

Broadly speaking, the theory of Fitting classes comprises a study of the classes themselves, a study of the embedding of those subgroups of an arbitrary group $g$ which lie in a particular Fitting class $\mathcal{F}$ (the $\mathcal{F}$-subgroups of $G$ ), and finally a study of the relation between $\mathcal{F}$ and the $\mathcal{F}$-subgroups of G. As in Formation theory we focus our attention on canonical sets of $\mathcal{F}$-subgroups of $G$.

If $\mathcal{X}$ is any $\mathrm{N}_{0}$-closed class, then in each. group $\mathrm{G}$ the set of normal $\mathfrak{X}$-subgroups has a unique maximal momber called the $\mathcal{X}$-radical of $G$, clearly characteristic in $G$ and denoted by $G_{\mathfrak{X}}$. For example $G_{\Re}$ is the Fitting subgroup of $G$. Suppose $N$ sn $G$, specifically $\mathbb{N}=N_{0} \triangleleft N_{1} \triangleleft N_{2} \triangleleft \ldots \triangleleft \mathbb{N}_{r}=G$. Then for $i=1, \ldots, r \quad\left(N_{i-1}\right)_{X}$ char $N_{i-1} \triangleleft N_{i}$, and so $N_{X}=\left(N_{0}\right)_{X} \triangleleft\left(N_{1}\right)_{X} \triangleleft\left(N_{2}\right)_{X} \triangleleft \ldots \triangleleft\left(N_{r}\right)_{X}=G_{X}$. Letting $\mathbb{N} \in \mathcal{X}$ we see that $G_{\mathfrak{X}}$ contains every subnormal $\mathfrak{X}$-subgroup of G. If now $\mathcal{F}$ is a Fitting class and $N$ sn $G$ then $N_{\mathcal{F}}=N \cap G_{\mathcal{F}}$. For, we have just seen that $N_{y} \leqslant G_{y}$, but certainly $N \cap G_{y} \triangleleft N$ and $N \cap G_{\mathcal{F}}$ sn $G_{\mathcal{F}}$, so $N \cap G_{\mathcal{F}} \in S_{n} \mathcal{F}=\mathcal{F}$. 
The characteristic of a Iitting class $\mathcal{F}$ (denotod by char $\mathcal{F}$ ), is defined to be the set $\pi$ of those primes $p$ which divide the order of some 7 -eroup. So of course $\mathcal{F} \leq \mathcal{S}_{\pi}$.

\subsubsection{Proposition (Hartley [4])}

If $\mathcal{F}$ is a Fitting class and char $\mathcal{F}=\pi$ then $\eta_{\pi} \subseteq \mathcal{F} \subseteq \mathcal{S}_{\pi}$. Proof

We need only prove $\eta_{\pi} \subseteq \mathcal{F}$. To do this it suffices to show that each cyclic $p$-group lies in $\mathcal{F}$ for all $p \in \pi$, since a nilpotent $\pi$-group is generated by such subgroups and they are of course subnormal. So let $p \in \pi$, then by derinition there exists a group $G \in \mathcal{F}$ such that $p|| G \mid$. Thercfore there exists a normal subgroup $H$ of $G$ such that $H$ has a normal subgroup $\mathrm{K}$ of index $\mathrm{p}$ in $\mathrm{H}$. Then $\mathrm{H} \times \mathrm{H} / \mathrm{K}$ is the product of normal subgroups $\{(h, 1): h \in H\}$ and $\{(h, K h): h \in H\}$, eacil isomorphic to $I$. Now $H \triangleleft G \in \mathcal{F}$, and so since $\mathcal{F}$ is a Fitting class, we have $H \times H / K \in \mathcal{F}$. It follows that $\mathcal{F}$ contains $\mathrm{H} / \mathrm{K} \cong \mathrm{C}_{\mathrm{p}}$. An easy induction argupment shows that $\mathrm{C}_{\mathrm{p}} \mathrm{n}$ is a subgroup of the repeated wreath product $\left(\ldots\left(C_{p} 2 C_{p}\right) 2 C_{p} \ldots\right) / C_{p}$ with $\mathrm{n}$ terms, which is clearly generated by its (necessarily subnormal) subgroups of order $p$. Again by subnormality we deduce $c_{p^{n}} \in \mathcal{F}$ and the proof is complete.

We now define the central object of our study. Let $\mathbb{X}$ be an arbitrary class of groups. A subgroup $V$ of $G$ is called an -injector of $\mathrm{G}$ if $\mathrm{V} \cap \mathrm{N}$ is a maximal $\mathfrak{X}$-subgroup of $\mathrm{N}$ for each subnormal subgroup $\mathbb{N}$ of $G$. We say $V \cap \mathbb{N}$ is $\underset{X \text {-maximal }}{\text { in }}$

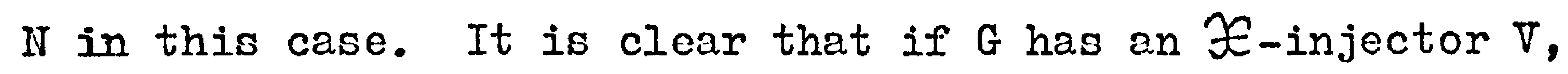
then the image $V^{\theta}$ of $V$ under an automorphism $\theta$ of $G$ is an $\mathfrak{X}$-injector of $\mathrm{G}$ also, and $\mathrm{V} \cap \mathbb{N}$ is an $X$-injector of $\mathrm{N}$ for each subnormal subgroup $N$ of $G$. 
The following theorem sets the theory of Pitting classcs in motion and was proved in 1967. For comploteness end futuro reforence we reproduce its elegant froof here.

\subsubsection{THEOREM (Fischer, Gascnütz and Hartley [5] Satz 1)} If $\mathcal{F}$ is a Fitting class, then each group $G$ has precisely one conjugacy class of $\mathcal{F}$-injectors.

PROOF

The argupment depends on the following key lemma.

\subsubsection{Lemma (Hortley [5] Lemna)}

Let $\mathcal{F}$ be a Fitting class and $G$ a group with $N \triangleleft G$ and $G / N \in \Re$. If $V_{1}$ and $V_{2}$ are $\mathcal{F}$-maximal suborolips of $G$ and $V=V_{1} \cap N=$ $V_{2} \cap N$ is $\mathcal{F}$-maximal in $N$, then $V_{1}$ and $V_{2}$ are conjugate in $G$. Proof

Clearly $V_{1}, V_{2} \leqslant N_{G}(W)=G^{*}$ say, and $G^{*} / N \cap G^{*} \in \mathcal{M}$, hence we may assume $W \triangleleft G$. For $i=1,2$ let $T_{i}=\mathbb{N}_{G}\left(V_{i}\right)$, then $\left[V_{i}, \mathbb{T}_{i}, \underline{T}_{i}, \ldots, \mathbb{T}_{i}\right] \leqslant \mathbb{N}$ eventually since $G / \mathbb{N} \in \mathcal{M}$. Of course $\left[v_{i}, T_{i}, T_{i}, \ldots, T_{i}\right] \leqslant v_{i}$, so $\left[V_{i}, T_{i}, T_{i}, \ldots, T_{i}\right] \leqslant N \cap v_{i}=W$ eventuaily, which means $V_{i} / W$ is a hypercentral normal subgroup of $T_{i} / H$. So $V_{i} / W$ lies in a system rormalizer of $T_{i} / W$, which in turn lies in a Carter subgroup of $\mathrm{T}_{i} / \mathrm{W}$, which we may write as $V C_{i} / W$ :for some Carter subgroup $C_{i}$ of $T_{i}$. Now $W C_{i} / W \in \Omega$, so $V_{i}$ is an $\mathcal{f}$-maximal subıormal subgroup of $W_{i}$ and therefore $V_{i}=\left(W C_{i}\right)_{\hat{j}}$. Suppose $x \in G$ normalizes $C_{i}$, then $x$ normalizes $w_{i}$ and hence a.zso its $\mathcal{F}$-radical $v_{i}$. Therefore $x \in T_{i}$, but $C_{i}$ is self-normalizine in $T_{i}$, so in fact $x \in C_{i}$. This shows $C_{i}$ is a Carter subgroup of $G$ and so $C_{1}$ and $C_{2}$ are conjugate in $G, C_{1}=C_{2}{ }^{E}$ say, so finally $V_{1}=\left(W C_{1}\right)_{r}=\left(W C_{2} g\right)_{r g}=\left(W C_{2}\right)_{F}^{g}$ $=\mathrm{V}_{2} \mathrm{~g}$. 
The theorem clearly holrs whon $|G|=1$, so ve use induction, supposing the result trine for all groups of order loss then $|a|$.

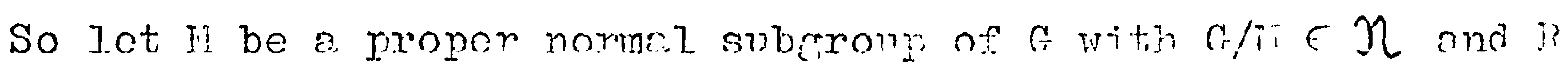
an $\mathcal{F}$-injector of $M$. We show that if $V$ is $\mathcal{F}$-maxima in $G$ vith $R \leqslant V$, then $V$ is an $\mathcal{F}$-injector of $G$. It will clecrly suffice to show that $V \cap G^{*}$ is an $\mathcal{F}$-injector of $G^{*}$ whonevor $G^{*}$ is a maximel normel. subgroup of $G$. Let $V^{*}$ be an $\mathcal{F}$-jnjector of $G^{*}$, this exists by induction. Let $N=G^{*} \cap M$, then $G / M \in \eta$, and furthormore $V \cap N$ and $V^{*} \cap N$ are $\mathcal{F}$-injectors of $N$. $B y$ induction the are conjugate, so (replacirg $\mathrm{V}^{*}$ by a conjugate if necessary) we assume $V \cap N=V^{*} \cap N=U$ say. Iow if $\bar{V}$ is an 7 -maximal subgroup of $G$ conteining $V^{*}$, then Iemma 1.1 .3 . implies that $\bar{V}$ and $V$ are conjugate in $G$. Ilence $\bar{V} \cap G^{*}=V^{*}$ and $V \cap G^{*}$ are too, which implies $V \cap G^{*}$ is an $\mathcal{F}$-injector of $\mathrm{G}^{*}$ as required.

Now suppose $V_{1}$ and $V_{2}$ are $\mathcal{F}$-injectors of $G$, and again that $G^{*}$ is a maximal normal subgroup of $G$. As injectors of: $G^{*}$, $V_{1} \cap G^{*}$ and $V_{2} \cap G^{*}$ are conjugate in $G^{*}$ by induction, and so as before we may actur.IJy assume they are equal. It is tinen an immediate consequence of Iemma 1.1.3. that $V_{1}$ and $V_{2}$ are conjugate in $G$, and the proof is complete.

Fundarnental use will be made of the following corollaries of 1.1 .2 and its proof.

1.1.4 THEORFI (Fischer, Gaschütz and Hartley [5] Korollax and Satz 2)

Let $\mathcal{F}$ be a Fitting class and $G$ a group. Then

a) If $N \triangleleft G$ with $G / N \in \eta$, and $V$ is an $\mathcal{F}$-maximal subgroup of $G$ sucin that $V \cap N$ is an $F$-injector of $N$, then $V$ is an F-injector of $G$. 
b) If $1==G_{0} \triangleleft G_{1} \triangleleft G_{2} \neg \ldots \triangleleft G_{r}=G$ and $G_{i} / G_{i-1} \in \eta$ for $i=1, \ldots, x$ then $V$ is an $\mathcal{F}$-injector of $G$ is and only if $V \cap G_{i}$ is $\mathcal{F}$-maximal in $a_{i}$ for $i=1, \ldots, r$.

c) If $V$ is an $F$-injector of $G$ and $V \leqslant H \leqslant G$ then $V$ is an $\mathcal{F}$-injector of $\mathrm{H}$.

\section{IROOF}

a) follows from the proof of 1.1 .2 and $b$ ) by induction. To prove c) suppose $1=G_{0} \triangleleft G_{1} \triangleleft \ldots \triangleleft G_{r}=G$ is a series with nilpotent factors. Then so also is $1=\mathrm{H}_{\mathrm{O}} \triangleleft \mathrm{H}_{1} \triangleleft \ldots$ $<\mathrm{H}_{\mathbf{r}}=\mathrm{H}$, where $\mathrm{H}_{i}=\mathrm{H} \cap \mathrm{G}_{i}$ for $i=1, \ldots, \mathrm{r}$. Now $\mathrm{H}_{i} \cap \mathrm{V}=$ $G_{i} \cap H \cap V=G_{i} \cap V$ is $F$-maximal in $G_{i} \cap H=H_{i}$, so by b) $V$ is an $\mathcal{F}$-injector of $\mathrm{H}$.

We shall denote the set of $\mathcal{F}$-injectors of a group $G$ by $I_{\mathfrak{F}}(G)$.

The proof of the following converse to 1.1 .2 is clear.

\subsubsection{Proposition}

If $X$ ic a class of groups such that each group $G$ has an $\mathcal{X}$-injector, then $\mathcal{X}$ is a Fitting class.

Considered together, $1,1.2$ and 1.1 .5 show that Fitting classes are precisely the classes for which injectors exist in all groups. This demonstrates that, despite what the. closure operations suggest, a Fitting class is best regarảed as the dual of a Schunck class [6]. Such classes are precisely those for which projectors exist in all group:. This point of view is strongly endorsed by Gaschuttz in [7] and [8].

The fact that $\mathcal{H}$-projectors (where $\mathcal{H}=Q \mathcal{H}$ ) are conjugate whenever they exist [9], prompted the offene Frage 1 of [5]: Are the $\mathfrak{X}$-injectors of a group $G$ (where $\mathfrak{X}=S_{n} \mathfrak{X}$ ) necessarily conjugate when they exist? 
The question ras closed in the negative by Havkes, and wo sketch his exmple here. It is worth noting that in [10] Chambers shows the answer is yes if $a$ satisfies the strong condition of having $p-$ length 1 for 2.1 primes $p$.

\subsubsection{Example}

There exists a group $G$ with the unique chief serios indicated.

Let $H$ be the non-Abelian group of order 21

and $\mathcal{X}$ the $S_{n}$-closed class it generates, so $X=\left\{\mathrm{H}, \mathrm{C}_{7}, 1\right\}$.

$N$ has complements $\mathrm{H}_{1}$ and $\mathrm{H}_{2}$ not conjugate

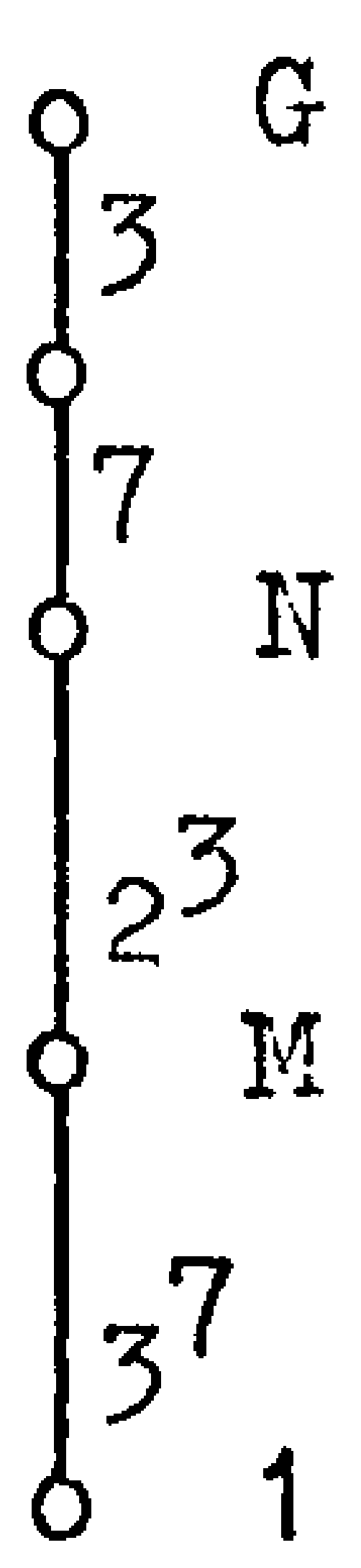
in $G$, both isomorphic to $H$, each of which is an $\mathcal{X}$-injector of $G$.

1.1.2 and 1.1 .4 have the following easy but important consequences.

\subsubsection{Proposition}

Let $\mathcal{F}$ be a Fitting class, $V$ an $\mathcal{F}$-injector of $G$ and $N \triangleleft G$.

a) $V \cap N$ is pronormal in $G$ and in particular $V$ pro $G$.

b) $G=N_{G}(V \cap N)$.

c) $V$ covers or avoids each chief factor of $G$.

d) If $\mathcal{F}$ has characteristic $\pi$, then an $\mathcal{F}$-injector $v_{0}$ of a Hall $\pi$-subgroup $G_{\pi}$ of $G$ is an $\mathcal{F}$-injector of $G$. Proof

a) Suppose $x \in G$, then $V \cap N$ and $(V \cap N)^{x}$ are $\mathcal{F}$-injectors of $N$. By 1.1 .4 c) they are $\mathcal{F}$-injectors of their join $J \leqslant N$, and therefore conjugate in $J$ by 1.1.2. This establishes $\mathrm{V} \cap \mathrm{N}$ pro $\mathrm{G}$.

b) This follows from the Frattini argupment (which may be 
applicd whonevor wo have $\mathrm{H}$ pro $\mathrm{G}$ and $\mathrm{I} \leqslant \mathrm{N} \rightarrow \mathrm{G}$, to rield $G=$ $\left.{ }^{I N T}{ }_{G}(\mathrm{H}).\right)$, and a).

c) If $\pi / T$ is a chies factor of $G$, then $b$ ) implies that $G$ normelizes $K(V \cap N)$, so $I(V \cap N)=N$ or $V \cap N \leqslant K$.

d) By the definjtion of characteristic, $V$ is a $\pi$-group and so $V^{X} \leqslant G_{\pi}$ for some $x \in G$. Then $V^{X}$ is an $\mathcal{F}$-injector of $G_{\pi}$ by 1.1.4. c), therefore is conjugate to $V_{0}$ by 1.1.2. Thus $V_{0}$ is an $\mathcal{F}$-injector of $G$ as required.

\subsection{Fischer classes and the sets IIff(G) and $\operatorname{IIT} f(G)$.}

If $\mathcal{F}$ is a Fitting class, an $\mathcal{F}$-subgroup 1 of $G$ is called a Fischer $\mathcal{F}$-subgroup of $G$ if $T$ cortains cach $\mathcal{F}$-subgroup of $G$ which it normalizes. This is cleerly equivalent to the condition that I' contains each normel $\mathcal{F}$-subgroup of any $\mathrm{H} \leqslant \mathrm{G}$ with $\mathrm{T} \leqslant \mathrm{H}$. Thus the Fischer $\mathcal{F}$-subgroups are dual to the $\not{X}$-covering subgroups of Gaschütz. ( $T$ is an $\mathscr{X}$-covering subgroup of $G$ if $T \in X$ and covers each $X$-quotient of any $H \leqslant G$ with $T \leqslant H_{.}$)

It is an immediate consequence of $1.1 .4 \mathrm{c}$ ) that an $\mathcal{F}$-injector of a group $G$ is also a Fischer 7 -subgroup of $G$. Thus denoting the set of Fischer $\mathcal{F}$-subgroups of $G$ by $I I_{\mathcal{F}}(G)$, we have $I_{\mathcal{F}}(G) \subseteq I I_{f}(G)$. It is one of the major disappointments of the theory that there exist $\mathcal{F}$ and $G$ where this inclusion is proper, that is the Fischer $\mathcal{F}$-subgroups of $G$ do not form a single conjugacy class, a disparity with the dual theory. (It is of course clear that in general $\operatorname{II}_{\mathcal{F}}(G)$ is a union of conjugacy classes of G.) The only known example of this is due to Dark [11], and we shall exhibit some Corollaries of this complicated work later. (See 2.7 and $4.1 .3 \mathrm{c}$ ).) 
However, if $\mathcal{F}=\$ \mathcal{F}$ then $I_{\mathcal{F}}(G)=I I_{\mathcal{F}}(G)$ for all $G$. In fact we have the following, userully stronger result, due to Fischer, a prooi of which apnears in [4]. A Fitting class. 7 is celled a Fischer class if

If $\triangleleft G \in \mathcal{F}, \mathbb{N} \leqslant \mathrm{H} \leqslant \mathrm{G}$ and $\mathrm{H} / \mathrm{H}$ has prime power order imply $\mathrm{H} \in \mathcal{F}$. So certainly an s-closed Fitting class is a Fischer class.

\subsubsection{THEOREM (Fischer)}

$\mathcal{F}$ is a Fischer class implies $I_{\mathcal{F}}(G)=\operatorname{II}_{\mathcal{F}}(G)$ for all $G$.

A further canonical set of $\mathcal{F}$-subgroups of a group $G$ are the $\mathcal{F}$-maximal subgroups of $G$ which contain the $\mathcal{F}$-radical of $G$. We denote this set by $\operatorname{III}_{\mathcal{F}}(G)$, and observe that each Fischer $\mathcal{F}$-suboroup of $G$ belongs to $\operatorname{III}_{\mathcal{F}}(G)$. Thus for arbitrary $\mathcal{F}$ and G we have

$$
I_{\mathcal{F}}(G) \subseteq \operatorname{III}_{\mathcal{F}}(G) \subseteq \operatorname{III}_{\mathcal{F}}(G)
$$

It is surprisingly frequently true of a Fitting class 7 that these three sets coincide for each $G$, that is, F-injectors are characterised as those $\mathcal{F}$-maximal subgroups containing the $\mathcal{F}$-radical. For example when $\mathcal{F}=\mathcal{X}$ (see 2.1.2), and of course when $\mathcal{F}=S_{\pi}$, for any set of primes $\pi$. (The Hall $\pi$-subgroups are the $\zeta_{\pi}$-injectors of $G$ ) However, interesting examples of this phenomena only occur when $\mathcal{F}$ has ' full characteristic ', as we prove in the next result.

1.2.2 Proposition

$I_{\mathcal{F}}(G)=\operatorname{III}(G)$ for all G implies $\eta \subseteq \mathcal{F}$ or $\mathcal{F}=S_{\pi}$ for some $\pi$. Proof

Suppose $\eta \nsubseteq \mathcal{F}$, that is there exists a prime $p \notin \pi=\operatorname{char} \mathcal{F}$. Let $G$ be an arbitrary $\pi$-group. Each normal subgroup of $B=$ $C_{p}{ }^{2 G}$ has order divisible by $p$, and so $H_{y}=1$. Now if $1 \neq G_{q}$ is a sylow $q$-subgroup of $G$, then $G_{q} \in \mathcal{F}$ since $q \in \pi=\operatorname{char} \mathcal{F}$. 
Therefore $G$ lies in some $\mathcal{F}$-maximal subcroup $H$ of $V$ which, by the triviality of $v_{f}$ and the hypothesis of the proposition, is an $\mathcal{F}$-injector of 1. This holds for all $q|| G \mid$ and therefore $|H| \geqslant|G|$. But of course $p h|H|$ since $p \Varangle \pi$ so $|I|=|G|$ and $G \in \mathcal{F}$. We deduce $S_{\pi} \subseteq \mathcal{F}$, and equality follows since $\pi=\operatorname{char} \mathcal{F}$.

This shows that if $\mathcal{F}$ is a Fitting class of characteristic $\pi$ and $\mathcal{F} \neq S_{\pi}$, then the question of the validity of $I_{\mathcal{F}}(G)=$ $\operatorname{III}_{\mathcal{F}}(G)$ is best asked for $G \in S_{\pi}$, the class of $\pi$-Grouxs. of course, for such $\mathcal{F}$, the $\mathcal{F}$-subgroups of an arbitrary group $G$ are just the $\mathcal{F}$-subgroups of the Hall $\pi$-subgroups of $G$. Thus the problem of the embedding of the $\mathcal{F}$-subgroups of $G$ divides into two, namely the embedding of the $\mathcal{F}$-subgroups of a IIaII $\pi$-subgroup $G_{\pi}$ in $G_{\pi}$ and the embedding of $G_{\pi}$ in $G_{\text {. Only the }}$ first problom is dependent on the Fitting class $\mathcal{F}$, so when $\mathcal{F}$ has characteristic $\pi$ it is natura. to work within the caterory of $\pi$-groups.

In order to produce an example which shows that the converse of 1.2.2 is false, we mention a well-known and useful technique for constructing groups with a unique chief series.

\section{2 .3 Lemma}

Let $\mathrm{H}$ be a group with a unique minimal normal subgroup wi where $M$ is a $q$-group. If $p$ is a prime distinct from $q$ then $H$ has a faithful irreducible representation over GF(p).

\section{Proof}

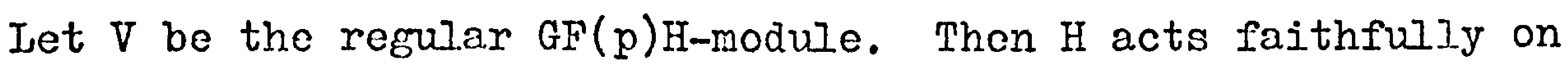
some composition factor of $V$. For, if not, $M$ (a.s the unique minimal normal subgroup of $\mathrm{H}$ ) lies in the kernel of the representation of $H$ on each composition factor of $V$ and 
therefore, by a well-lnown result, must act trivially on $V$ since $a \neq p$. This contradicts the fact that $V$ is the resular $G$ GP $(p)$ H-module, wo our assertion holds and ve heve a feithful irreducible Ga(p) H-module.

\section{2 .4 Corollary}

Let $p_{n}, \ldots, p_{2}, p_{1}$ be a sequence of primes with no two consecutive terms equal. Then there exists' a group $G$ with a unique chier sories $1=G_{n} \triangleleft G_{n-1} \triangleleft \ldots \triangleleft G_{2} \triangleleft G_{1} \triangleleft G_{0}=G$, and elementary Abelian subgroups $E_{n}, \ldots, E_{2}, E_{1}$ such that $\mathbb{F}_{i}$ is a $p_{i}$-group which covers the chief factor $G_{i-1} / G_{i}$ and avoids the rest, and $E_{i}$ normalizes $E_{j}$ for all $1 \leqslant i \leqslant j \leqslant n$, so $G=E_{n} E_{n-1} \cdots E_{2} E_{1}$.

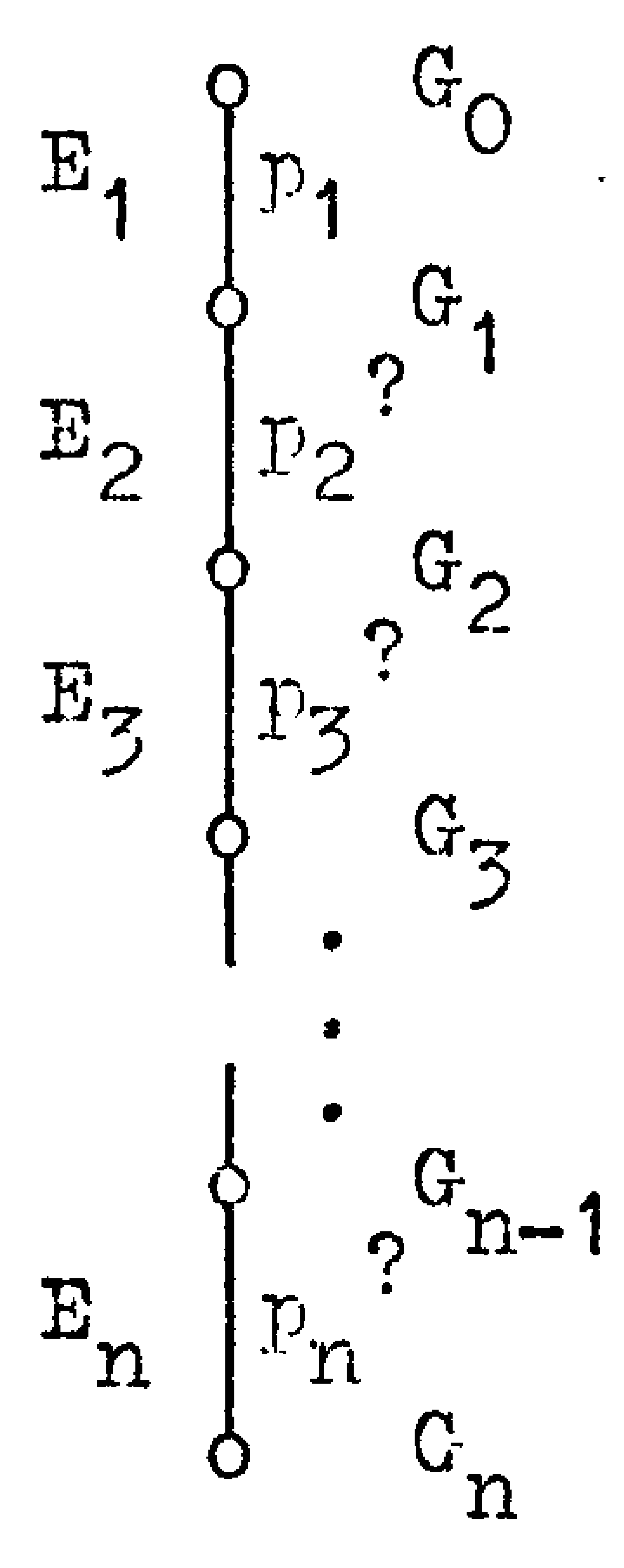

Proof

We use induction on $n$. If $n=1$ put $G=E_{1} \cong C_{p_{1}}$. By induction suppose $H=E_{n-1} \ldots E_{2} E_{1}$ has the recuired properties. Then $\mathrm{H}$ has a unique minimal normal subgroup $\mathrm{E}_{\mathrm{n}-1}$ which is $\mathrm{a}$ $p_{n-1}$-group. By Jemma 1.2 .3 there exists a faithful irreducible $G F\left(p_{n}\right) H$-module and we let $E_{n}$ be its additive group. Clearly the split extension $G=E_{n} H$ satisfies the statement of the Corollary, and for each $i=1, \ldots, n, G / G_{i}$ is a primitive soluble group with unique minimal nomal subgroup $G_{i-1} / G_{i}$ which is self-centralizing in $G / G_{i}$. Inowledge of familiar small groups and their representations usually indicates the orders of the first few terms in the series $E_{1}, E_{2}, \ldots$

To specify the Fitting class in our example, we need the concept of the product of two Fitting classes, introduced by Gaschütz in [7]. 
If $\mathcal{F}_{1}$ and $\mathcal{F}_{2}$ are Fitting clesses, coschüto definos $\mathcal{F}_{1} \mathcal{F}_{2}=\left\{\mathrm{G}: G / G_{y_{1}} \in \mathcal{F}_{2}\right\}$. This is a subcjess of

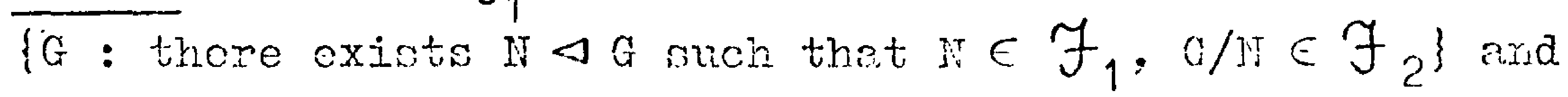
the inclusion can be strict, but it is easily seen that the classes coincide when $\mathcal{F}_{2}=0 \mathcal{F}_{2}$. Certainly $\mathcal{F}_{1} \subseteq \mathcal{F}_{1} \mathcal{F}_{2}$, but $\mathcal{F}_{2} \Phi_{1} \mathcal{F}_{1} \mathcal{F}_{2}$ can occur. Vie now porform the routine verification of some elementary facts concorning $\mathcal{F}_{1} \mathcal{F}_{2}$, returning to a fuller discussion in 2.6 .

\subsubsection{Proposition}

If $\mathcal{F}_{1}, \mathcal{F}_{2}, \mathcal{F}_{3}$ are Fitting classes then

a) $\mathcal{F}_{1} \mathcal{F}_{2}$ is a Fitting class of characteristic char $\mathcal{F}_{1} \cup \operatorname{char} \mathcal{F}_{2}$

b) $G_{F_{1} F_{2}} / G_{\mathfrak{F}_{1}}=\left(G / G_{\mathcal{F}_{1}}\right)_{\mathcal{F}_{2}}$ for all $G$

c) $\left(\mathcal{F}_{1} \mathcal{F}_{2}\right) \mathcal{F}_{3}=\mathcal{F}_{1}\left(\mathcal{F}_{2} \mathcal{F}_{3}\right)$.

\section{Proof}

a) If $\mathbb{N} \triangleleft G \in \mathcal{F}_{1} \mathcal{F}_{2}$, then $\mathbb{N} / \mathbb{N}_{\mathcal{F}_{1}}=\mathbb{N} / \mathbb{N} \cap G_{\mathcal{F}_{1}} \cong \mathbb{N T G} G_{\mathcal{F}_{1}} / G_{\mathcal{F}_{1}}$ and $\mathbb{N G}_{\mathfrak{F}_{1}} / G_{\mathcal{F}_{1}} \triangleleft G / G_{\mathcal{F}_{1}} \in \mathcal{F}_{2}$, hence $\mathbb{N} \in \mathcal{F}_{1} \mathcal{F}_{2}$. If $\mathcal{F}_{1} \mathcal{F}_{2} \ni \mathrm{N}_{1}, \mathrm{~N}_{2} \triangleleft \mathrm{N}_{1} \mathrm{~N}_{2}=G$ then $G / G_{r_{1}}=\mathrm{N}_{1} \mathrm{~N}_{2} / G_{F_{1}}=$ $\mathrm{N}_{1} G_{F_{1}} / G_{\mathcal{F}_{1}} \cdot \mathbb{N}_{2} G_{F_{1}} / G_{F_{1}}$ and $N_{i} G_{F_{1}} / G_{F_{1}} \cong N_{i} / N_{i} \cap G_{\mathcal{F}_{1}}=N_{i} /\left(N_{i}\right)_{\mathcal{F}_{1}} \in \mathcal{F}_{2}$, so $G \in \mathcal{F}_{1} \mathcal{F}_{2} \cdot c_{p} \in \mathcal{F}_{1} \mathcal{F}_{2}$ if and only if $c_{p} \in \mathcal{F}_{1} \cup \mathcal{F}_{2}$, gives the rest of the statement.

b) As we have remarked above, $\mathcal{F}_{1} \subseteq \mathcal{F}_{1} \mathcal{F}_{2}$, so $G_{F_{1}} \leqslant G_{\mathcal{F}_{1}} \mathcal{F}_{2}$. But certainly $\left(G_{\mathfrak{F}_{1}} \mathcal{F}_{2}\right) \mathcal{F}_{1} \unlhd G$, therefore we must have $G_{F_{1}}=\left(G_{F_{1} F_{2}}\right)_{F_{1}}$. So $G_{F_{1} F_{2}} / G_{F_{1}}$ is a normal $\mathcal{F}_{2}$-subgroup of $G / G_{\mathcal{F}_{1}}$, that is $G_{\mathcal{F}_{1} \mathcal{F}_{2}} / G_{\mathcal{F}_{1}} \leqslant\left(G / G_{\mathcal{F}_{1}}\right)_{\mathcal{F}_{2}}=\mathbb{N} / G_{F_{1}}$ say. Again $\mathbb{N}_{\mathcal{F}_{1}} 4 G$ so $\mathbb{N}_{F_{1}}=G_{F_{1}}$ and $N \in F_{1} \mathcal{F}_{2}$, which yields the result. c) $G \in\left(\mathcal{F}_{1} \mathcal{F}_{2}\right) \mathcal{F}_{3} \Leftrightarrow G / G_{F_{1} \mathcal{F}_{2}} \in \mathcal{F}_{3} \Leftrightarrow\left(G / G_{\mathcal{F}_{1}}\right) /\left(G / G_{F_{1}}\right)_{\mathcal{F}_{2}} \in \mathcal{F}_{3}$ $($ by $b)) \Leftrightarrow a / G_{F_{1}} \in \mathcal{F}_{2} \mathcal{F}_{3} \Leftrightarrow G \in \mathcal{F}_{1}\left(\mathcal{F}_{2} \mathcal{F}_{3}\right)$. 
Iet $\mathcal{F}=\varkappa \zeta_{3}$ and $\mathrm{c}$ a Emoup constructed as

in 1.2.1 from the sequence $2,5,3,2$.

Then $E_{4}=G \mu S_{3}$ and using 1.1 .4 b) we see

that $\mathrm{E}_{A_{i}} \in \mathrm{I}_{\boldsymbol{F}}(\mathrm{C})$.

However $E_{4} E_{1} \in \operatorname{III}(G)-\operatorname{IIF}_{\mathcal{F}}(G)$.

Thus $\chi \subseteq \mathcal{F}$, but $\operatorname{IF}_{\mathcal{F}}(G) \neq \operatorname{IIIF}_{\mathcal{F}}(G)$.

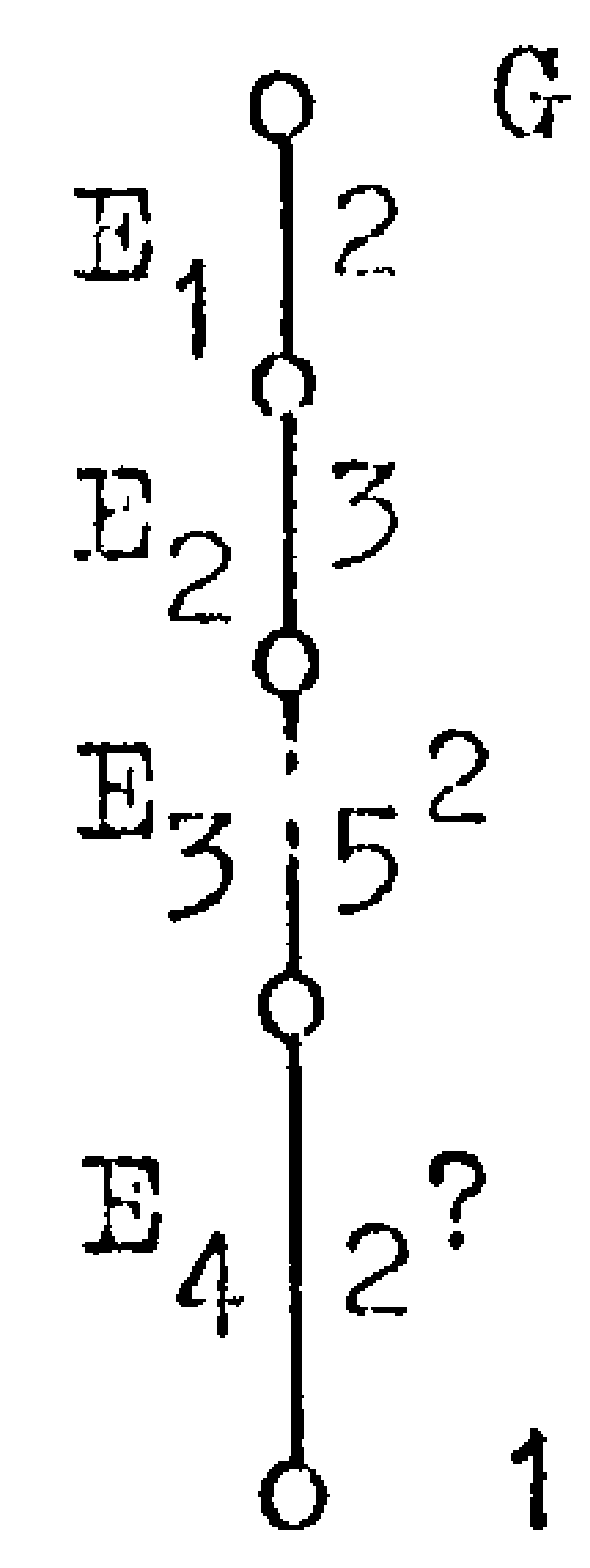

Using similar techniques it is easy to construct a group

G for which $\operatorname{III}_{f}(G)$ comprises arbitrarily many conjugracy classes.

We eird this introductory chapter with an elementary lemma and some cautionary observations.

\section{2 .7 Lemma}

Suppose $F, K \leqslant G, H \perp K$ and that the Hall $\pi$-subgroup $G_{\pi}$ of $G$ reduces into $H$ and $K$. Then $G_{\pi} \searrow H K, H \cap K$ and

$\left(H \cap G_{\pi}\right)\left(R \cap G_{\pi}\right)=H K \cap G_{\pi}$.

\section{Proof}

Clearly $\left(H \cap G_{\pi}\right)\left(K \cap G_{\pi}\right) \subseteq H K \cap G_{\pi}$

and $\left|\left(H \cap G_{\pi}\right)\left(K \cap G_{\pi}\right)\right|=\left|H \cap G_{\pi}\right|\left|K \cap G_{\pi}\right| /\left|H \cap K \cap G_{\pi}\right|$

Now $|H K|=|H||K| /|I \cap \cap K|$ and $G$ reduces into $H$ and $K$,

so taking $\pi$-divisors we have

$|H K|_{\pi}=\left|H \cap G_{\pi}\right|\left|K \cap G_{\pi}\right| /|H \cap K|_{\pi}$

By (1) $\left|\left(H \cap G_{\pi}\right)\left(K \cap G_{\pi}\right)\right| \leqslant\left|H K \cap \cap G_{\pi}\right| \leqslant|H K|_{\pi}$

and of course $\left|\mathrm{H} \cap \mathrm{K} \cap \mathrm{G}_{\pi}\right| \leqslant|\mathrm{H} \cap \mathrm{K}|_{\pi}$

Then (2) and (3) show that we must have equality in (4) and (5) which implies the statement of the lemma.

\subsubsection{Proposition}

Suppose $\mathcal{F}$ is a Tittine class for which $N_{1}, N_{2} \triangleleft G=N_{1} N_{2}$ and $V=\mathcal{F}$-injector of $G$ imply $V=\left(N_{1} \cap V\right)\left(N_{2} \cap V\right)$. Then $F=S_{\pi}$ for some set of primes $\pi$. 
Let $\pi=$ char $F$ and supnose $F+S_{\pi}$. Thet $I$ be a group of minimal order in $\mathcal{S}_{\pi}-\mathcal{F}$. So viy $=F$ say, is the unjuge maximal nomel suberoup of $N$, of index $p$ say. By assumption $\mathrm{N} \notin \mathcal{F}$, so $F$ is $\mathcal{F}$-maximal in $N$ and therefore its unique $\mathcal{F}$-injector. $N$ is a $\pi$-oroup, so $p \in$ char $\mathcal{F}$ and therefore $N / F \cong C_{p} \in \mathcal{F}$. Now $G=I N \times N / F$ is the product of normal suteroups $\{(n, 1): n \in \mathbb{N}\}$ and $\{(n, F n): n \in N\}, N_{1}$ and $N_{2}$ say, each isomorphic to $\mathbb{N}$ and each having $\{(f, 1): f \in F\} \cong F$ as its unique $F$-injector. Thus if $V$ is an $F$-injector of $G$, by hypothesis $V=\left(\mathrm{H}_{1} \cap \mathrm{V}\right)\left(\mathrm{N}_{2} \cap \mathrm{V}\right)$, so $\mathrm{V}=\{(f, 1): \pm \in F\}$. However $F \times C_{p} \in \mathcal{F}$, so $V$ is not $\mathcal{F}$-maximal in $G$, a contradiction giving $\mathcal{F}=\mathcal{S}_{\pi}$.

This proposition shows why $\left\{G: \mathcal{F}_{1}\right.$-injector of $\left.G \in \mathcal{F}_{2}\right\}$ (where $\mathcal{F}_{1}$ and $\mathcal{F}_{2}$ are Fitting classes), can fail to be a Fitting class when $\mathcal{F}_{1} \neq \mathcal{S}_{\pi}$ for some $\pi$. For example $G=\Sigma_{3} \times C_{2}$ is the product of two normal subgroups isomorphic

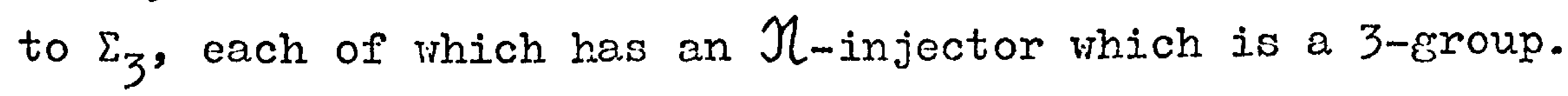
However $G$ itself does not enjoy this property.

\subsubsection{Proposition}

suppose $\mathcal{F}$ is a non-trivial Fitting class for which $\mathrm{N}_{1}, \mathrm{~N}_{2} \triangleleft \mathrm{G}=\mathrm{N}_{1} \mathrm{~N}_{2}$ implies $\mathrm{G}_{\mathcal{F}}=\left(\mathrm{N}_{1}\right)_{\mathcal{y}}\left(\mathrm{N}_{2}\right)_{\mathcal{F}}$. Then $\mathcal{F}=S$. Proof

We may repeat the proof of the previous proposition to show that $\mathcal{F}$ necessarily has the form $\mathcal{S}_{\pi}$, for some set of primes $\pi$. Now suppose $p$ and $q$ are primes such that $p \in \pi$ but $q \notin \pi$. Let $N$ be a group with unique chief series constructed in the manner of 1.2 .4 from the sequence $p, q, p$. Then $N_{f}=M$ say, the unique minimal normal subgroup of $N . G=N \times C_{p}$ is the product of two normal copies of $N$ (in the usual way), each 
with $f$-radicul $\mathrm{I} \times 1$. Wowover it is cipperont thet $\mathrm{C}_{\mathrm{f}}=\mathrm{J} \times \mathrm{C}_{\mathrm{p}}$, which contridicts our hypothosis. Thus our supposed choice of prines is not possible and ve deduce that $f$ is trivial or cqual to $\mathcal{S}$.

We shal]. even see in Chapter 5 that there exist Fitting classes $\mathcal{F}$ for which $(A \times B)_{\mathcal{F}}=A_{\mathcal{F}} \times B_{\mathcal{F}}$ can be false.

These observations are in sharp contrast to the situation when $\mathcal{F}$ is a saturated formation. For it is easily scen that if $G$ is a subdirect product of croups $\Lambda$ and $B$, then an $\mathcal{F}$-projector (respectively $\mathcal{F}$-normejizer) of $G$ is a subdirect product of the $\mathcal{F}$-projectors (rospectively $\mathcal{F}$-normalizers) of $A$ and $B$. 
In this chaptor vo shell try to indicate noat of the known Fitting classes, a promise whjch in itself reveals that our supply of examples is very limited. Contributing to this sparseness, many of the techniques for deriving new formations and Schunck classes from old ones fajl to dualize. In particular no satisfactory 'Iocal definition' for a Fitting class has yet emerged. Here we examine some established 'new from old' procedures, including the product $\mathcal{F}_{1} \mathcal{F}_{2}$, and introduce a new one, paying special attention to discovering the properties which the new class inherits from the old. However we enphasise that fundamental examples of Fitting classes, the building blocks for these procedures, are for from plentiful.

\subsection{The nilpotent injectors}

We begin with a mild ceneralization of a construction, due we undcrstand to Dade but also published by Nann, which

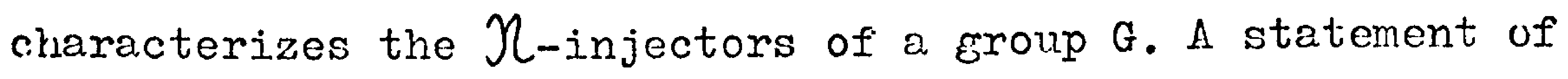
the characterization also appears on page 705 of Huppert's book.

Let $\left\{\pi_{i}\right\}_{i} \in I$ be a family of pairvise disjoint sets of primes (so certainly $I$ is countable and possibly finite), and put $\pi=\bigcup_{i \in I} \pi_{i}$ and $\pi_{i}^{\prime}=\pi-\pi_{i}$ for each $i \in I$. Let $\mathcal{X}$ be the class of those $\pi$-groups which have a normal Hall $\pi_{i}$-subgroup for each $i \in I$, so every $X$-group is a direct product of $\pi_{i}$-groups for various $i$. $X$ is clearly a Fitting class, in fact $\tilde{X}=\bigcap_{i \in I} \rho_{\pi_{i}} S_{\pi_{i}^{\prime}}$

2.1 .1 THEORENI

If $G \in \mathcal{S}_{\pi}$ then $I_{\mathfrak{X}}(G)=\operatorname{III} I_{X}(G)=\left\{\prod_{i \in I} V_{\pi_{i}}: V_{\pi_{i}} \in \operatorname{HaII}_{\pi_{i}}\left(C_{G}\left(G \eta_{\pi_{j}^{\prime}}\right)\right)\right\}$ 


\section{$\underline{P R O O T}$}

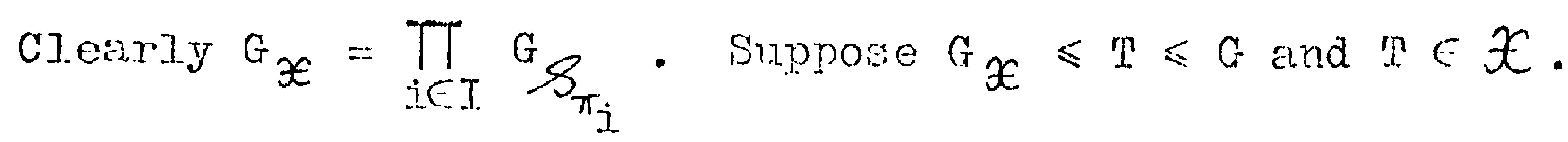
So $T_{\pi_{i}}$, the unique Hall $\pi_{i}$-subcroup of $\mathrm{T}$, centralizos ${ }^{1 j} \pi_{i}^{\prime}$ and $T_{\pi_{i}^{\prime}} \geq \prod_{j=i} G^{G} S_{\pi_{j}}$. Now $\prod_{j \neq j} G_{\pi_{j}} \geq G_{\eta_{\pi_{i}^{\prime}}}$, and so ${ }^{T} \pi_{i}$ centralizes $G_{\pi_{\pi_{j}^{\prime}}}$. Let $V_{\pi_{i}}$ be a Hall $\pi_{i}$-subcroup of $\mathrm{C}_{\mathrm{G}}\left(\mathrm{G}_{\boldsymbol{N}_{\pi_{i}}}\right)$ containing $\mathrm{T}_{\pi_{i}}$. Iotice $\mathrm{V}_{\pi_{i}} \geq \mathrm{G}_{n_{\pi_{i}}}$.

Siuppose this selection has been made for each $i \in I$. If $i, j \in I$ vith $i \neq j$, then $\left[V_{\pi_{i}}, V_{\pi_{j}}\right] \leqslant C_{G}\left(G_{n_{\pi_{i}}}\right) \cap c_{G}\left(G_{n_{\pi !}}\right)$ which lies in $C_{G}\left(G_{n}\right)$ since $\pi_{i}^{\prime} \cup \pi_{j}^{\prime}=\pi$. As is well-linow $C_{G}\left(G_{n}\right) \leqslant G_{n}$, so $\left[v_{\pi_{i}}, V_{\pi_{j}}\right] \leqslant G_{n}$. In particular $V_{\pi_{i}}$ normalizos $G_{n} V_{\pi_{j}}$. Now by a previous remark and the construction of $V_{\pi_{j}}$ it follows that $G_{n} V_{\pi_{j}}=$ $G_{n_{\pi_{j}}} \nabla_{\pi_{j}}$, of which $V_{\pi_{j}}$ is the $S_{\pi_{j}}$-radical, a characteristic subgroup. Thus $V_{\pi_{i}}$ actually normalizes $V_{\pi_{j}}$, so by symmetry $\left[V_{\pi_{i}}, V_{\pi_{j}}\right] \leqslant V_{\pi_{i}} \cap V_{\pi_{j}}=1$. We have shom $T \leqslant \prod_{i \in I} v_{\pi_{i}}=V$ say. Clearly $\mathrm{V} \in \mathcal{X}$, so it remains to prove that all subgroups constructed in the manner of $V$ are conjugate in $G$.

$G$ is a finite group, so there are a finite number $n$ say, of non-trivial subgroups in the set $\left\{G_{\pi_{i}}: i \in I\right\}$. We may suppose the labelling makes $G_{\delta_{\pi_{1}}}, \ldots, G_{\delta_{\pi_{n}}} \neq 1$. Obviously $G_{\pi_{i}}=1$ iff $G_{n_{\pi_{i}}}=1$ and furthermore $V_{\pi_{i}}=1$ iff $G_{\pi_{i}}=1$. For if $G_{\eta_{\pi_{i}}}=1$ then $G_{\eta_{\pi_{i}^{\prime}}}=G_{\eta}$ which contains its centralizer in $G$, so $V_{\pi_{i}}=1$. The converse is clear from $V_{\pi_{i}} \geq G_{n_{i}}$. 
Let $\mathrm{V}=\mathrm{V}_{\pi_{1}} \times \mathrm{V}_{\pi_{2}} \times \ldots \times \mathrm{V}_{\pi_{n}}$ and $\mathrm{V}^{*}=\mathrm{V}_{\pi_{1}}^{*} \times \mathrm{V}_{\pi_{2}}^{*} \times \ldots \times \mathrm{V}_{\pi_{n}}^{*}$ be subrroups of G rith $V_{\pi_{i}}, V_{\pi_{i}}^{*} \in$ IIelI $_{\pi_{i}}\left(C_{G}\left(G n_{\pi_{i}}\right)\right)$ for $i=1, \ldots n$. Clearly there exists $x_{1} \in G$ such that $v_{\pi_{1}} x_{1}=V_{\pi_{1}}^{*}$. is an induction hypothesis, suppose there exists $x_{r} \in G$ such that $v_{\pi_{i}}{ }^{x_{r}}=v_{\pi_{i}}^{*}$ for $i=1, \ldots, x<n$. Now $v_{\pi_{r+1}}$ and $v_{\pi_{r+1}^{*}}^{*}$ are Hall $\pi_{r+1}$-suberoups of $\mathrm{C}_{G}\left(G_{n_{\pi^{\prime}+1}^{\prime}}\right) \cap \mathrm{C}_{G}\left(V_{\pi_{1}}^{*} \times \ldots \times V_{\pi_{r}}^{*}\right)$, so there exists $y \in C_{G}\left(V_{\pi_{1}}^{*} x \cdots V_{\pi_{r}}^{*}\right)$ such that $V_{\pi_{r+1}} x_{r} y=v_{\pi_{r+1}}^{*}$. Then $v_{\pi_{i}} x_{r} y=\left(v_{\pi_{i}}^{*}\right)^{y}=v_{\pi_{i}}^{*}$ for $i=1, \ldots, r$, and putting $x_{r} y=x_{r+1}$, we have $v_{\pi_{i}}^{x_{r+1}}={v_{\pi_{i}}^{*}}_{\text {for } i=1, \ldots, r+1}$. The induction step is complete and we deduce $\mathrm{V}^{\mathrm{x}} \mathrm{n}=\mathrm{V}^{*}$.

Whon $\pi_{i}=\left\{p_{i}\right\}$ the set consisting of the $i$ th prime for each positive integer $i, X=\Re$. So we have the immediate special case. :

\subsubsection{THEORTI (Fischer-Dade)}

$$
I_{n}(G)=\operatorname{III}_{n}(G)=\left\{\prod_{p|G|} \nabla_{p}: V_{p} \in \operatorname{SyI}_{p}\left(C_{G}\left(G_{n_{p}}\right)\right)\right\} \text { for all G. }
$$

Fischer, in [1], was the first to prove (though by a far more complicated arguфment) that all the $N_{\text {-maximal subgroups }}$ of a group $G$ which contain the Fitting subgroup are conjugate, and Gaschütz has dosignated thesc duals of the Carter suberoups the 'Fischer subgroups' of $G$. To avoid confusion with the previously defined Fischer $\mathcal{F}$-subgroups, we shall adhere to the term $K_{\text {-injector. }}$

Now suppose that, for each $i \in I, \mathcal{F}_{i}$ is a Fitting class of characteristic $\pi_{i}$, where as before $\left\{\pi_{i}\right\}_{i \in I}$ is a family of pairrise disjoint sets of primes. Again let $\pi_{i}=\bigcup_{i \in I} \pi_{i}-\pi_{i}$. clearly $\mathcal{F}=\bigcap_{i \in I} \mathcal{F}_{i} S_{\pi !}$ is a Fitting class comprising those 
$\pi$-croups which have a nomen wath $\pi_{j}$-subroup lyine in $\mathcal{F}_{j}$, for als. $i \in I$. fs a second corollary of 2.1 .1 we have :

$2.1 .3 \quad$ IITPONTI

If $G \in S_{\pi}$ then $I_{f}(G)=\left\{\prod_{i \in I} H_{\pi_{i}}: I_{\pi_{i}}\right.$ is an $\mathcal{F}_{i}$-injector of $v_{\pi_{j}}$ where $v_{\pi_{i}}$ is a IIaIl $\pi_{i}$-subgroup of $\left.c_{G}\left(c_{n_{\pi_{i}}}\right)\right\}$.

PROOP

Let $I I=\prod_{i \in I} H_{\pi_{i}} \leqslant \prod_{i \in I} V_{\pi_{i}}=V$ say, be subcroups of $G$ constructed as in the statement. Ici $T$ be an $\mathcal{F}$-injector of $G, T=\prod_{i \in I} T T_{i}$ say. Certainly $\eta_{\pi} \subseteq \mathcal{F}$, so $G_{n} \leqslant T$ and thercfore $T_{\pi_{j}}$ centralizes ${ }^{G_{\pi_{i}}}$. Let ${ }_{\pi_{i}}$ be a Hall $\pi_{i}$-subgroup of $\mathrm{C}_{G}\left(c_{n_{\pi_{i}}}\right)$ containing $\mathrm{T}_{\pi_{i}}$. Then $T \leqslant \prod_{i \in I} W_{i}=\forall$ say. By $\left.1.1 .4 \mathrm{c}\right) \mathrm{T}$ is an $\mathcal{F}$-injector of $\mathrm{V}$ and by 2.1.1 V $=w^{x}$ for some $x \in G$. It follows that $T^{x}$ is an $\mathcal{F}$-injector of $v$. Now $v_{\pi_{i}} \triangleleft v$ and the $\mathcal{F}$-subgroups of $v_{\pi_{i}}$ are preciscly its $\mathcal{F}_{i}$-subgroups, so $v_{\pi_{i}} \cap T^{X}=T_{\pi_{i}} x$ is actualiy an $\mathcal{F}_{j}$-injector of $v_{\pi_{i}}$. Thus for each $i \in I$ there exists $\mathrm{y}_{i} \in \mathrm{v}_{\pi_{i}}$ such that $\mathrm{H}_{\pi_{i}} \mathrm{y}_{i}=\mathrm{T}_{\pi_{i}} \mathrm{x}$. Notice that $\mathrm{y}_{j}$ centralizes $v_{\pi_{i}}$ whenever $i \neq j$, so putting $y=\prod_{i \in I} y_{i}$ we have $H_{\pi_{i}} y=T_{\pi_{i}}{ }^{x}$, and hence $H^{\mathrm{Y}}=\mathrm{T}^{\mathrm{X}}$. We have shown that a fixed injector is conjugate to any subgroup consiructed in the manner of $\mathrm{HI}$ which completes the proof.

\subsection{A class due to Gaschütz.}

For the duration of this section let $\mathcal{H}$ be a fixed but arbitrarily chosen Fitting class of characteristic $\pi$ say. If $p$ is a prime, Gaschutz defincs $e_{p}(\mathcal{H})=\{G$ : a]I p-chief factors of $\mathrm{G}$ between 1 and $\mathrm{G}_{\mathcal{H}}$ are central $\}$. Given two 


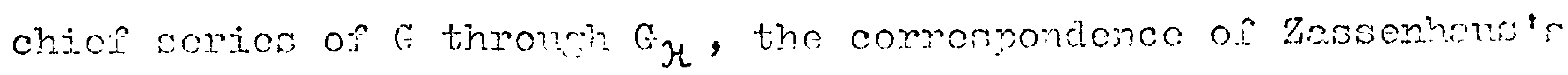
Jerme mkes factors between 1 and $G_{H}$ correngond, so $G \in e_{p}(\mathcal{H})$ if all the p-chicf factors betreen 1 and $c_{H}$ in a porticulor chief series of $G$ are centrel. Ti $p \uparrow \pi$ then the centratity condition is vacuous and $e_{p}(\mathcal{H})=\mathcal{S}$, so we shall almoy assume that $p \in \pi$.

\subsubsection{Proposition}

$e_{p}(\mathcal{H})$ is a Fittine class and actually a Fischer class. Proof

For simplicity mite $\mathcal{F}=e_{p}(\mathcal{H})$.

Suppose $N \triangleleft G \in \mathcal{F}$. Consider a chief series of $G$ through $\mathbb{N}_{\mathcal{H}}=\mathbb{N} \cap G_{\mathcal{H}}$ and $G_{\mathcal{H}}$. Then by hypothesis all p-chief factors of $G$ below $I_{\mathcal{H}}$ are central and it follows immediately that the p-chief factors of $N$ below $N_{\mathcal{H}}$ in a refinement of this series are central in $\mathbb{N}$, so $N \in \mathcal{F}$.

Now suppose $\mathcal{F} \ni \mathrm{N}_{1}, \mathrm{~N}_{2} \triangleleft \mathrm{G}=\mathrm{N}_{1} \mathrm{H}_{2}$. An easy induction argupment shows that, to prove $G \in \mathcal{F}$, we may assume that $\mathbb{N}_{1}$ and $\mathrm{N}_{2}$ are maximal nomel subgroujs of $\mathrm{G}$. Let $\mathrm{N}=\mathrm{N}_{1} \cap \mathrm{N}_{2}$ so $G / N$ is Abeliar. Consider a chief sories of $G$ through $N_{\mathcal{H}}$ and $G_{\mathcal{H}}$. The chief factors between $\mathbb{I}_{\mathcal{H}}$ and $G_{\mathcal{H}}$ are $G$-isomorphic to factors between $N$ and $G$, so are central. $\Lambda$-chief factor cf $G$ below $\mathrm{N}_{\mathcal{H}}$ is complctely reducible as $\mathrm{N}_{i}$-module by Clifford's theorem, and hence by the assumption $\mathbb{N}_{i} \in \mathcal{F}$ is central in $N_{i}$. It is therefore central in $G$ and we have $G \in \mathcal{F}$ as required.

To prove $\mathcal{F}$ is a Fischer class ve suppose $i \bar{G} \in \mathcal{F}$ and $N \leqslant H \leqslant G$ with $H / N$ a $q$-rroup for some prime $q$ (possibly $p=q$ ) and we must show $H \in \mathcal{F}$. Whatever $q$ is, the chief factors of $\mathrm{H}$ between $\mathrm{H}_{\mathcal{H}}=\mathrm{N} \cap \mathrm{H}_{\mathcal{H}}$ and $\mathrm{H}_{\mathcal{H}}$ are $\mathrm{H}$-isomornhic to factors between $N$ and $H$, so are central as $H / N \in \mathcal{S}_{q}$. Since $G \in \mathcal{F}$, the p-chief factors in a chief sories of $G$ beloi $N_{\mathcal{H}}$ are central, so the 
p-chief roctors of if in a refinemont of this sorics belor rix are centret. in $\mathrm{II}$.

Iin sumpose $\sigma$ is o subset of $\pi$ and dotine o $\sigma(\mathcal{H})=\bigcap_{p \in \sigma} e_{p}(\mathcal{H})$. Agrain set $\mathcal{F}=e_{\sigma}(\mathcal{H})$ for simplicity. It follows innizdiately from the definition that tho intersoction of a colnection of Fischer closses is elso a Fischer elass, so by Rischer's theorem 1.2.1, the previous proposition yjelds $I_{\mathcal{F}}(G)=I_{\mathcal{F}}(G)$ for all G. However still more is tme as we prove in the $\therefore$ ollowing result.

2.2 .2 THEOPTIST

$I_{\mathfrak{f}}(G)=\operatorname{III}_{\mathfrak{f}}(G)$ for a... $G$.

PROOF

As in 2.1.1 we identify the mombers of $\operatorname{III}_{\mathcal{F}}(G)$ and exhjbit their conjugacy, so we must first look for $G_{f}$.

(1) $\left(G_{\mathcal{H}}\right)_{\mathcal{F}}=G_{\mathcal{H}} \cap G_{\mathcal{F}}=\left(G_{\mathcal{H}}\right)_{S_{\pi-\sigma}} \varkappa_{\sigma}=\mathrm{K}$ say.

Obviously $\left(G_{\mathcal{F}}\right)_{\mathcal{H}}=\left(G_{\mathcal{H}}\right)_{\mathcal{F}}=G_{\mathcal{H}} \cap G_{\mathcal{F}}$ holds for arbitrary $\mathcal{F}$ and $\mathcal{H}$.

In our case a normal $\mathcal{F}$-subgroup of $G_{\mathcal{H}}$ has all its $\sigma$-chieI factors central, so is $p$-nilpotent for all $p \in \sigma$, and hence lies in $K$. On the other hand $K \in{ }^{\prime} f$ is clear.

Put $I=\left(G_{\mathcal{H}}\right)_{S_{\pi-\sigma}}$ and $C=C_{G}(K / I) \triangleleft G$

(2) $K=G_{\mathcal{H}} \cap K C$ and $G \triangleright K C \in \mathcal{F}$.

For, $K / I=\left(G_{H} / I\right)_{x}$ by $(1)$ and $\left.1.2 .5 \mathrm{~b}\right)$,

wo $\mathrm{K} / \mathrm{I}$ contains its centralizer in

$G_{\mu} / I$. Therefore $G_{\mu} \cap C \leqslant K$ and so

$K=K\left(G_{H} \cap C\right)=G_{H} \cap K C$ by the

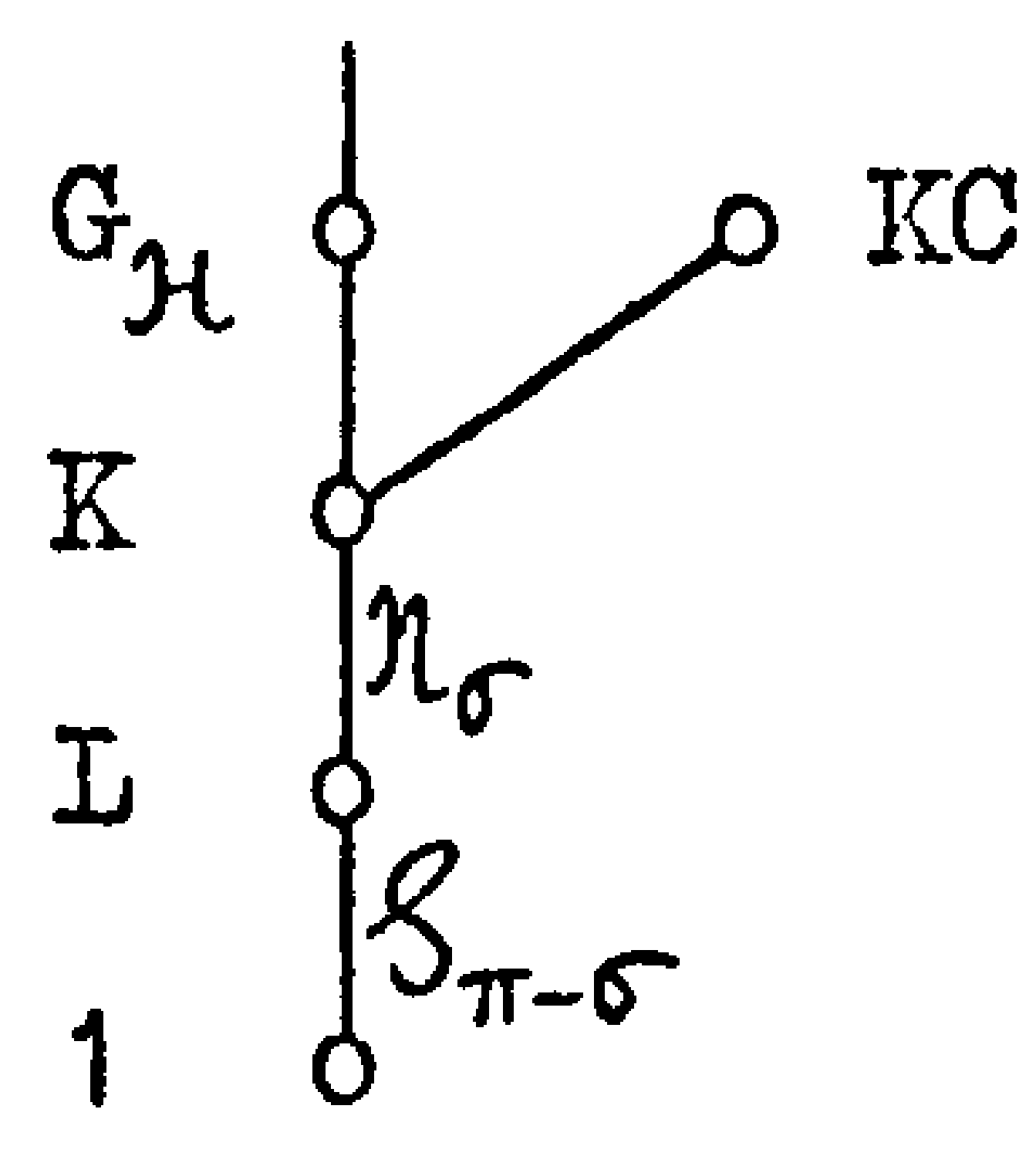

Dedekind Iav. Thus $(\mathrm{KC})_{\varkappa}=\mathrm{K}$ and $\mathrm{KC} \in \mathcal{F}$

follows immediately from the definition of $\mathcal{F}$.

(3) If $K C \leqslant \mathrm{~T} \in \mathcal{F}$ then $T_{p}$ centralizes $O_{p^{\prime}}(K / I)$ and $T / K C$ is a $\sigma$-group. 
Here $p$ is any prime end, as elweys, ${ }^{p} p$ denotes a sylow p-subsroup of $T$. TH certeinly contains $K$, so all chier factors of $\mathrm{T}$ betroen $I$ and $\mathrm{K}$ are contrel. In wrticuler $\mathrm{T}$ centralizes all the chief fectors of $T$ betwcen $I$ and $O_{p},\left(K \bmod I_{1}\right)$ and we deduce as usual thet $T_{p}$ centralizes $\mathrm{O}_{p^{\prime}}(\mathrm{K} / \mathrm{I})$. So if $\mathrm{p} \notin \sigma$, then $\mathrm{T}_{\mathrm{p}}$ centralizes $\mathrm{K} / \mathrm{I}$ and therefore rjes in $C$. Hence $\mathrm{p} / \mathrm{KC}$ is a $\sigma$-group.

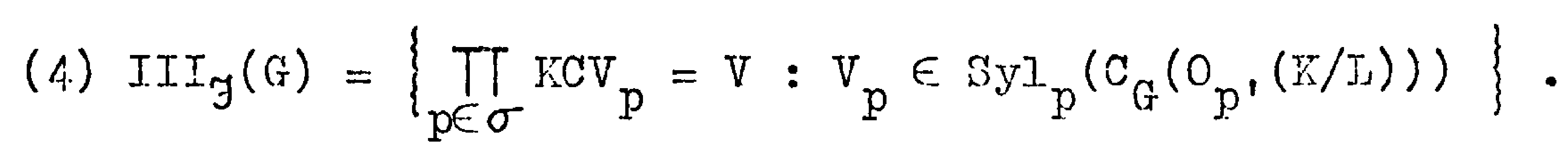
Suppose $K C \leq T \in \mathcal{F}$. By (3), T/KC is a $\sigma$-group and for each $p \in \sigma \quad s_{p} \leqslant V_{p}$ for some $V_{p} \in S y I_{p}\left(C_{G}\left(O_{p}(K / I)\right)\right)$. If $p, q \in \sigma$ and $p \neq q$ then $\left[V_{p}, V_{q}\right] \leqslant C_{G}\left(O_{p},(K / I)\right) \cap C_{G}\left(O_{q},(K / I)\right)=C$. Letting $\bar{G}=G / K C$, this means $\bar{V}_{p}$ and $\bar{V}_{q}$ centralize each other

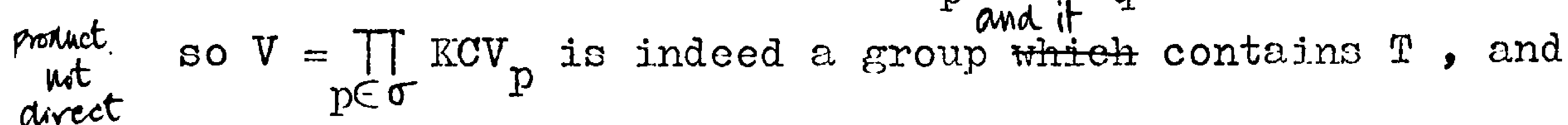
furthermore $V / K C \in M$. Since $K C \leqslant G_{\mathcal{F}}$, to prove (4) it will suffice to show that $V \in \mathcal{F}$.

$V_{\mathcal{H}} \cap K C=(K C)_{\mathcal{H}}=K$ by (2). So chief factors of $\mathrm{V}$ between $\mathrm{K}$ and $\mathrm{V}_{\mathcal{H}}$ are $\mathrm{V}$-isomorphic to factors between $\mathrm{KC}$ and $\mathrm{V}$, which are central since $V / K C \in \eta$. If $p \in \sigma, o_{p}(K / L)$ is centralized by $C$ and by each $V_{q}$ with $q \neq p$, so $V$ induces a p-group of automorphisms on each p-chief factor of $\mathrm{V}$ between $\mathrm{I}$ and $\mathrm{K}$,

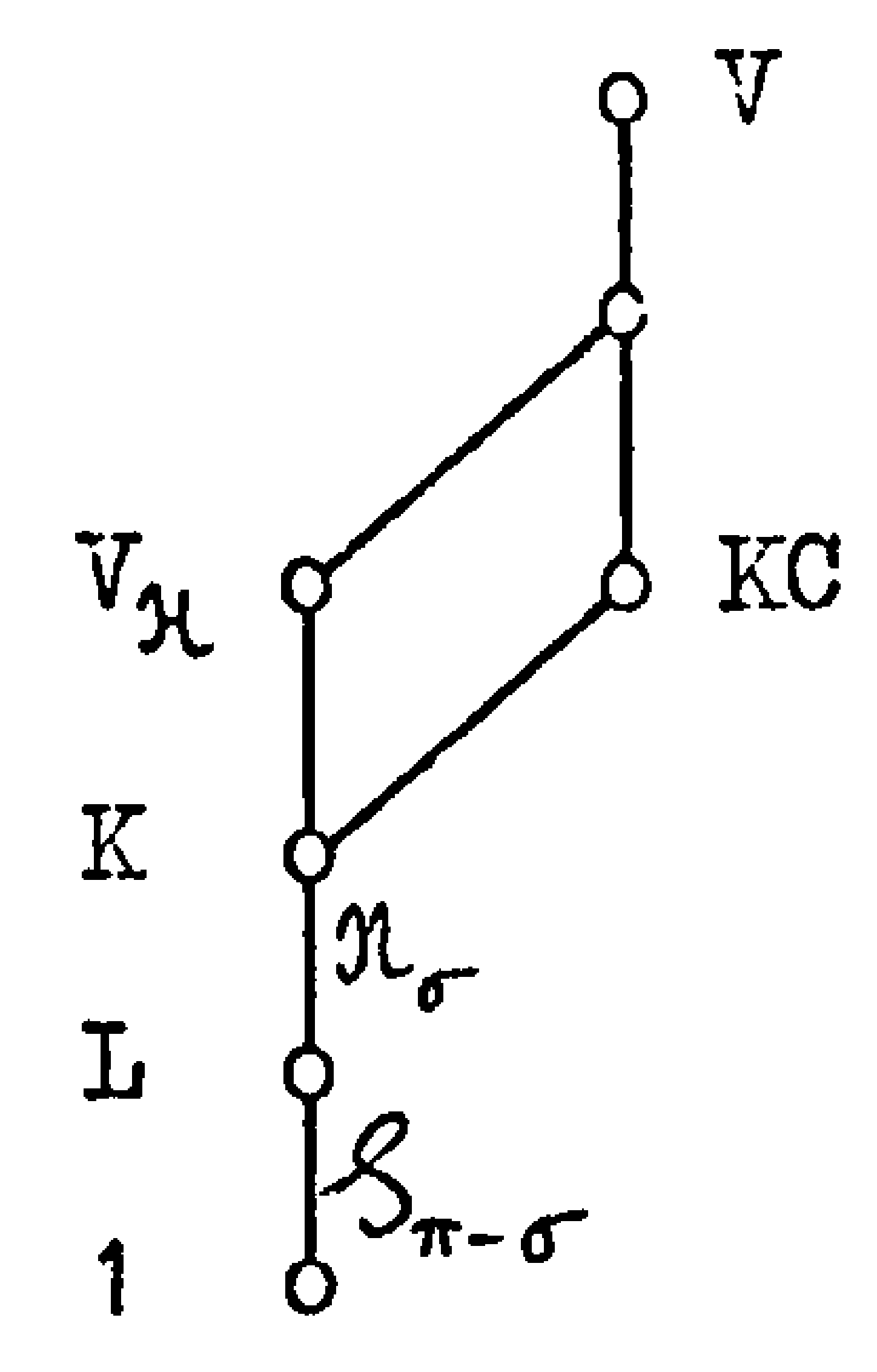
hence such a chief factor must actually be central in $V$. $I$ is a $\sigma^{\prime}$-group so we have $v \in \mathcal{F}$. (5) $I_{\mathcal{F}}(G)=\operatorname{III}_{\mathcal{F}}(G)$

In view of (4) it is sufficient to show that all the subgroups $\mathrm{V}$ are conjugate. Now $\bar{V}_{p}$ is a sylow $p$-subgroup of the normal subgroup $\bar{C}_{G}\left(O_{p^{\prime}}(K / I)\right)$ of $G$ and we have seen $\left[\bar{V}_{p}, \vec{V}_{q}\right]=1$. 
It folpows as in the proos of ?.1.1 thet e.7. the subrromg are conjurato in $\bar{G}$ and tbororore sinco $r C \leqslant V$, aly tho $V$ are conjugte in G. (iye shell see in chanter 3 thet conjugecy in such situations follows from a more coneren result.)

Prom the proof opent it is appent :

(6) $G_{y}=\prod_{n \in \sigma} O_{p}\left(Y C_{C}\left(O_{p},(T / T)\right) \bmod \mathrm{KC}_{\mathrm{i}}\right)$

\subsubsection{Pyariple}

As in example of a class of this type, consider $e_{p}\left(S_{p}\right)$. It is not difficult to show that this class is precisely $\left\{G: O_{p}(G)\right.$ is centrolized by all the $p^{\prime}$-elements of $\left.G\right\}$. Furthermore $e_{3}\left(S_{3}\right)$ contains $\Sigma_{4}$ but not its subgroup $\Sigma_{3}$, so $e_{3}\left(S_{3}\right)$ is a Pischer class (by 2.2.1) which is not S-closed.

Notice that for the classes in both 2.1 .1 and 2.2 .2 , it happens that an injector of an arbitrary group $G$ hes sylow subgroups which are also sylow subgrorps of certain nomal subgroups of G. This proporty is in fact a consequence of the classes being Fischer clesses, a result due to Fischer himself, and we give a proof in Chapter 3. However, example 1.2.6 shows that $I_{\mathcal{F}}(G)=\operatorname{III}_{\mathcal{F}}(G)$ does not follow when $\mathcal{F}$ is a Fischer class or even an S-closed class.

\subsection{Groups vith central socle}

We now turn our attention to a non-Fischer class. If $p$ is a prime, let $\mathcal{F}_{\mathrm{p}}$ denote $\{\mathrm{G}$ : each minimal normal p-subgroup

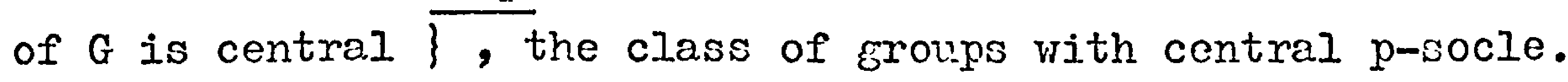
clearly $\Re_{\subseteq} \mathcal{F}_{p}$. We suspect that this class has its origins in Kicl too.

2.3 .1 Proposition

$\mathcal{F}_{\mathrm{p}}$ is a Fitting class. 
Let $I$, be a minime I nomel p-sucroup of $i t$, where $f i d G \in F_{n}$.

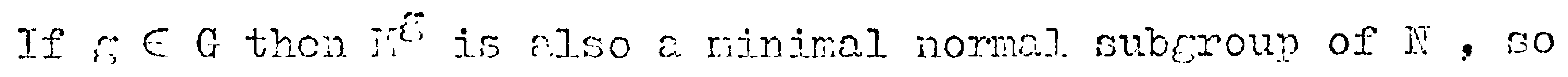

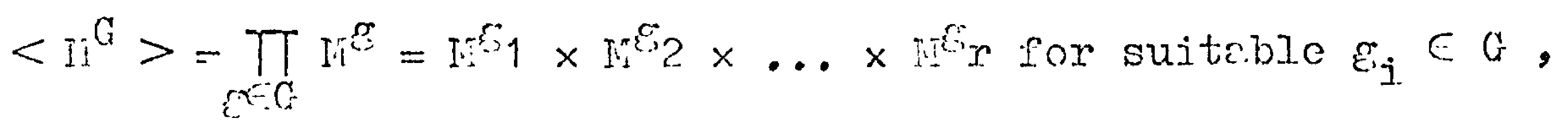
and is a p-roun. By hypothosis, a minimal normel subroup of $G$ contained in $<{ }^{G} T^{G}>$ is central in $G$, so there exists $x=m_{1} m_{2} \ldots m_{r} \in Z(G)$ such thet $m_{i} \in I r_{i}^{E_{i}}$ and $m_{j} \neq 1$ for some $j$. low if $n \in I$ then $x^{n}=m_{1}{ }^{n} m_{2}{ }^{n} \ldots m_{r}^{n}$ and $x=x^{n}$ since $x \in z_{1}(G)$, so $m_{i}{ }^{n}=m_{i}$ for all $n \in N$ and for all $i$ because the product of the If is direct. Thercfore $1 \neq m_{j} \in \mathbb{I}^{6} j \cap Z(I I)$, but $Z(\mathrm{IN}) \triangleleft G$, so $\mathrm{H} \cap Z(\mathrm{JJ}) \neq 1$ and by minimality $\mathrm{H} \leqslant Z(\mathrm{~N})$. So we have $N \in \mathcal{F}_{p}$.

Now let $\mathrm{I}$ be a minimal. normel subgroup of $G$, where $G=N_{1} N_{2} \triangleright N_{1}, N_{2} \in \mathcal{F}_{p}$. If $N \cap N_{1} \neq 1$ then $N \cap N_{1}$ contains a minimal normal suberoup of $\mathrm{H}_{1}$ which by hypothesis is central in $\mathrm{N}_{1}$. Thus $\mathrm{H} \cap \mathrm{Z}\left(\mathrm{N}_{1}\right) \neq 1$, but certeinly $\mathrm{Z}\left(\mathrm{N}_{1}\right) \triangleleft \mathrm{G}$, so by minimality $\mathbb{M} \leqslant z\left(\mathbb{N}_{1}\right)$. If $M \cap N_{1}=1$ then $\left[M, N_{1}\right]=1$. In either case $\mathrm{H}_{1}$ (and similarly $\mathbb{N}_{2}$ ) centralizes $\mathbb{N}$, so $\mathbb{H} \leqslant(G)$. Therefore $G \in \mathcal{F}_{p}$, completing the arguфment.

\subsubsection{Example}

$\mathcal{F}_{3}$ is not a Fischer class. For let $\mathrm{II} \cong \mathrm{C}_{3} \times \mathrm{C}_{3}$

be the natural module (under right action) for the subgroup $A=<\left[\begin{array}{l}11 \\ 01\end{array}\right],\left[\begin{array}{l}20 \\ 01\end{array}\right]>\left(\cong \Sigma_{3}\right)$ of $G_{1}(2,3)$, and put $G=M A$.

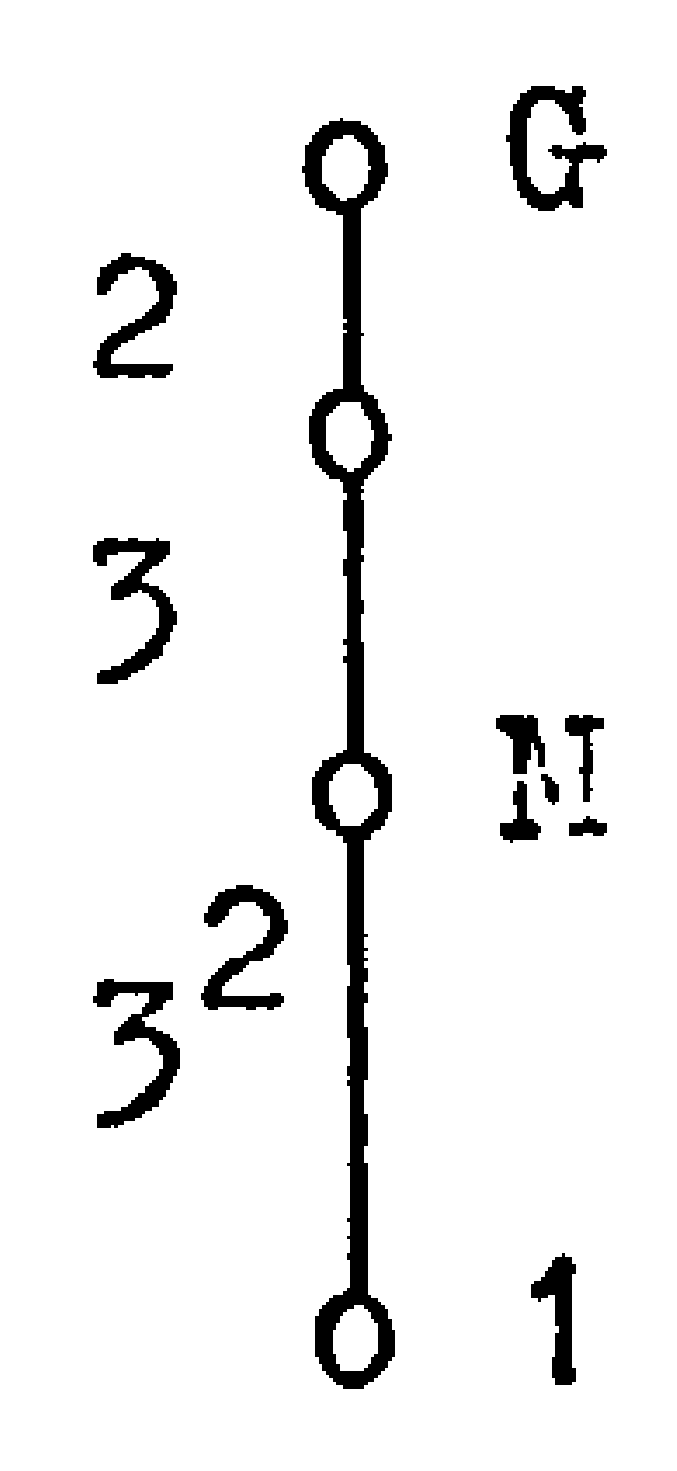

I has a unjque minimal A-admissable subgroup, which is central, so $G \in \mathcal{F}_{3}$. However, $\mathrm{MA}_{2}$, an extension of $M$ by a group of order 2, clearly does not lie in $\mathcal{F}_{3}$.

\subsubsection{Proposition}

For each $G, G_{F_{p}}=C_{G}(p-\operatorname{soc} G)$. 
Iroof

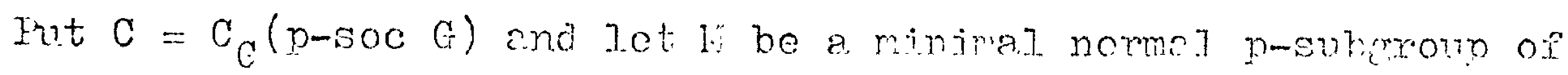

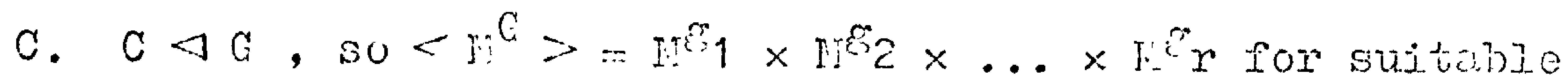
$\tilde{\sigma}_{i} \in G$, end $\mathbb{N} \xi_{i}$ irs rinimal. normal in $C$. Nor a minimal normal subgroup of $G$ contained in $<{ }^{G}>$ is contral in $C$, and it follows as in the proof of ?.3.1 thet $\mathrm{l}$ is contral in $C$. Thus $c \in \mathcal{F}_{p}$ and so $c \leqslant G_{\mathcal{F}_{p}}$.

On the other hand, each mimimal. nomel p-subrroup $n$ of G lies in $G_{F_{p}}$, and therefore a minimal normal subgroup of $G_{F_{p}}$ sontained in $N$ lies in $Z\left(G_{\mathcal{F}_{p}}\right)$. Thus $N \cap Z\left(G_{\mathcal{F}_{p}}\right)+1$ and by minimality it follows that $G_{F_{p}}$ centralizes $\mathbb{N}$. Hence $G_{F_{p}} \leqslant C . \square$

If $\pi$ is a set of primes let $\mathcal{F}_{\pi}$ denote $\bigcap_{p \in \pi} \mathcal{F}_{p}$, the class of groups with central $\pi$-socle. (We hope that the context will prevent confusion arising from our basic convention that $\mathcal{F}_{\pi}=\mathcal{F} \xi_{\pi}$ ) clearly: $G_{\mathcal{F}_{\pi}}=\bigcap_{p \in \pi} G_{\mathcal{F}_{p}}=\bigcap_{p \in \pi} C_{G}(p-\operatorname{soc} G)($ by 2.3 .3$)=C_{G}(\pi-\operatorname{soc} G)$. In particular if $\Omega$ denotes the set of a.lI primes, $G_{\mathcal{F}_{\Omega}}=\mathrm{C}_{\mathrm{G}}$ (soc $G$ )

We gather from Professor Gaschütz that the folloring result has also been obtained by one of his students.

\section{3 .4 THEOREM} For each $G I_{F_{p}}(G)=\operatorname{III}_{F_{p}}(G)=\left\{C_{G}\left(C_{P}(G)\right): P=p-\operatorname{soc} G_{F_{p}}\right.$ and
$\left.G_{p} \in \operatorname{SyI}_{p}(G)\right\}$.

PROOF

As in 2.3 .3 let $C=C_{G}(p-$ soc $G)=G_{F_{p}}$. so $P=p-$ soc $C$. (1) $C=C_{G}(F)$

$c \in \mathcal{F}_{p}$, so certainly $c$ centrajizes $P$, that is $c \leqslant C_{G}(P)$. Each minival normel p-subgroup of $G$ is central in $C$, so 


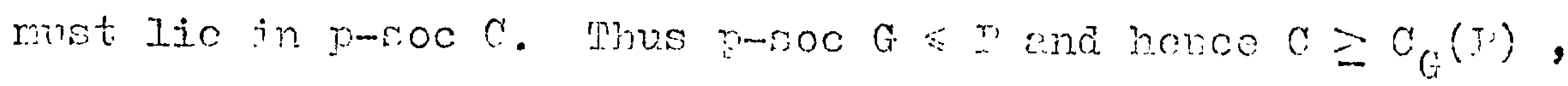
giving equality

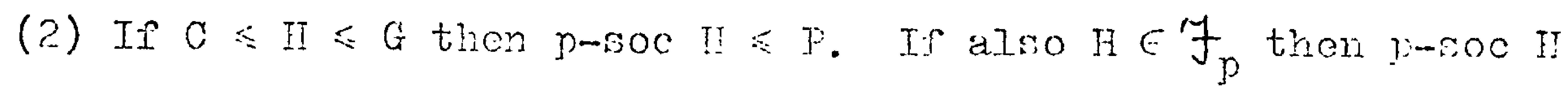
$=C_{P}(I)$.

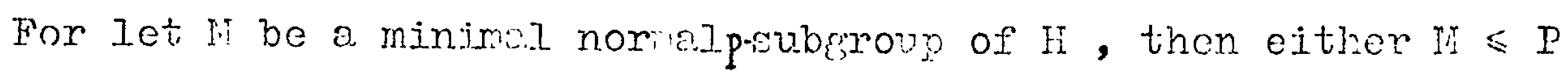
or $I: \cap P=1$. In the sccond cose $l$ contralizos ' $P$ and theresore lies in 6 by (1). Mhen. Clifford's theosem tells us that Ii is completely reducible under the cotion of $C$, so $I \leqslant P$ arter all. If also $H \in \mathcal{F}_{p}$, then $p-s 0 c H \leqslant C_{p}(I I)$ follows from what we have just proved. The reverse inclusion holds because $P$ is elemontary Abelian.

(3) If $C \leqslant H \in \mathcal{F}_{p}$ then $p-$ soc $H=C_{p}(H)=C_{P}\left(H_{p}\right)$ where $I_{p} \in$ $\operatorname{Syl}_{p}(\mathrm{Fi})$

In view of (2) it suficices to prove $C_{p}(H)=C_{p}\left(H_{p}\right)$, which $\mathrm{VC}$ do by induction on $|H / C|$, the statement being okvious when $H=C$. Tet $N$ be a marimal normal subgroup of It containing $C$. Jet $N_{p}$ $=H_{p} \cap \mathrm{H}$. Then using our induction hypothesis we have :

$$
C_{P}(N)=C_{P}\left(N_{p}\right) \geq C_{P}\left(H_{p}\right) \geq C_{P}(H) \text {. }
$$

a) If $|\mathrm{H}: \mathrm{N}| \neq \dot{\mathrm{p}}$, then $\mathrm{C}_{\mathrm{P}}$ (IN) is normalized byr $\mathrm{II}$, and by Maschke's theorem is completely reducible under its action. So since $H \in \mathcal{F}_{p}$ we have $C_{P}(H) \leqslant C_{p}(H)$, and hence equality holds throughout * .

b) If $|H: N|=p$, then (from *) $C_{P}\left(H_{p}\right)$ is centralized by both $\mathrm{N}$ and $\mathrm{H}_{\mathrm{p}}$, and therefore also by their product $\mathrm{H}$. So again $\mathrm{C}_{\mathrm{p}}\left(\mathrm{H}_{\mathrm{p}}\right) \leqslant \mathrm{C}_{\mathrm{P}}(\mathrm{H})$ and equality follors immediately.

(4) If $c \leqslant H \in \mathcal{F}_{p}$ then $c_{G}(p-\operatorname{soc} H) \in \mathcal{F}_{p}$.

Certainly $\mathrm{H} \leqslant \mathrm{C}_{\mathrm{G}}(\mathrm{p}-\mathrm{soc} \mathrm{H})=\underset{\text { the argument }}{\mathrm{T}}$ say. Now let $\mathrm{II}$ be a minimal normal. p-subgroup of $T$. Then $M \leqslant P$ by $_{\Lambda}(2)$, so $I$ contains a minimal normal subgroup of $\mathrm{H}$ which, by definition, is contral in $T$. 


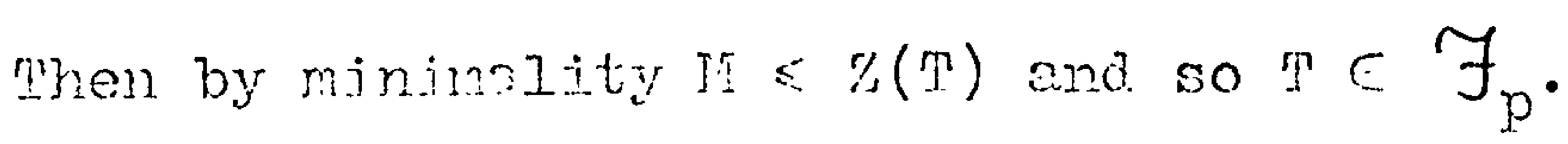

(5) $\operatorname{III}_{\mathcal{F}_{p}}(a)=\left\{C_{G}\left(C_{p}\left(G_{p}\right)\right): C_{p} \in S_{Y I}(G)\right\}$

Suppose $G_{\mathcal{F}_{p}}=C \leqslant I \in \mathcal{F}_{p}$ and $\delta y I_{p}(H) \Rightarrow I_{p} \leqslant G_{p} \in S y I_{p}(G)$.

Thon using (3) p-soc $H=C_{p}\left(I I_{p}\right) \geqslant C_{P}\left(G_{p}\right)$

Iow by (1) $\mathrm{CG}_{p}$ induces a p-group of outomorphisms of $\mathrm{P}$ so irom

(2) it is clear that $C G_{p} \in \mathcal{F}_{p}$, and so $p-\operatorname{soc}\left(C C_{p}\right)=C_{p}\left(G_{p}\right)$.

Then by $(4)$ we heve $C_{G}\left(C_{F}\left(G_{p}\right)\right) \in \mathcal{F}_{p}$. Thus from ** we acduce $I \leqslant C_{G}(n-s \circ C H) \leqslant C_{G}\left(C_{P}\left(G_{p}\right)\right) \in \mathcal{F}_{p}$, which establishes (5).

Conjugacy of the iylow subgroups of $G$ now implies $\operatorname{ITI}_{F_{p}}(G)$ is a conjugecy class, giving the statement of the theorem. $\square$

We have been unable to prove an analogous result for the class $\mathcal{F}_{\pi}$, but we suspect that $I \mathcal{F}_{\pi}(G)=\operatorname{III} \mathcal{F}_{\pi}(G)$ for alI $G$ can be established using similer techniques. In particular $(1)-(4)$ of the proof of 2.3 .4 hold with $p$ replaced by $\pi$ and $P$ by $S=\pi-\operatorname{soc~} \mathrm{G}_{\mathcal{F}_{\pi}}$, so the problem is to determine a suitable replacomont for $G_{p}$ in $(5)$. At least we have :

\section{3 .5 THEORTII}

For each G $\operatorname{IF}_{\pi}(G)=\operatorname{II}_{\mathcal{F}_{\pi}}(G)$.

PROOF

Example 2.3 .2 shows $\mathcal{F}_{\pi}$ need not be a Fischer class, so we are denied an immediate proof by Fischer's theorem 1.2.1. Hovever, we can argue from the structure of a group $G$ of minimal order which contains a non-injective Fischer $\mathcal{F}_{\pi}$-subirroup $\mathrm{E}$, a stmucture which Fischer uses in the proof of his theorem. Proofs of the details we require $((1)-(3)$ below) appear in section 2.3 of $[4]$.

Consider such a group $G$ and subgroup $E$. Let $F=G_{F}$, then $\mathrm{F}=\mathrm{C}_{\mathrm{G}}(\mathrm{R})$ where $\mathrm{R}=\pi$-soc $\mathrm{G}$, by the remark following 2.5 .3 . 
$F$ is not an $\mathcal{F}_{\pi}$-injector of $G$, for othorriso F rould bo

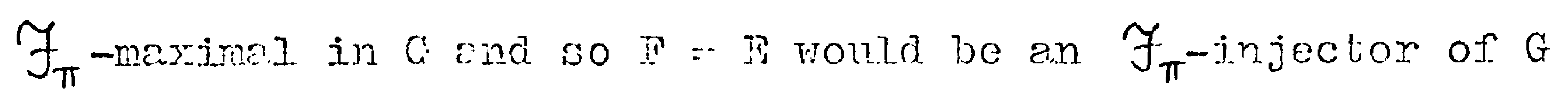

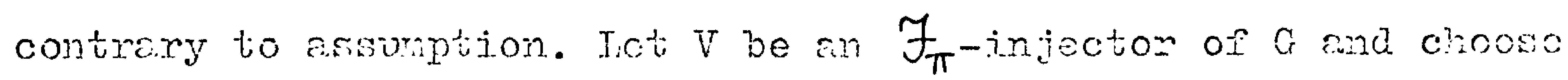
a nomal subgroup $H$ of $G$ containing $F$ and minimal subject to $\mathrm{V} \cap \mathrm{H}>\mathrm{T}$. Let $\mathrm{K}$ be maximel amons the nomel subcromps of $\mathrm{G}$ lying between $F$ and $H$. Then by mininality of $I I, F i 心$ $\mathcal{F}_{\pi}$-maxinal in $K$, so $V \cap K=E$. BJ 1.1 .7 2) $V$ covors or arojes the chief factors of $G$, so it covers $I / Y$ since $V \cap I I>F$. Furthermore :

(1) With $\mathrm{H}$ chosen, $\mathrm{K}$ is unique.

(2) $\mathrm{HE}=\mathrm{G}$ and $\mathrm{H} \cap \mathrm{E}=\mathrm{F}$.

(3) $\mathrm{H} / \mathrm{K}$ is the unique minimal. normal subgroup of $G / K$.

Suppose $H / K$ is a p-chief factor of $G$. Let $J / H$ be a chief factor of G. From (2) and (3) it follows

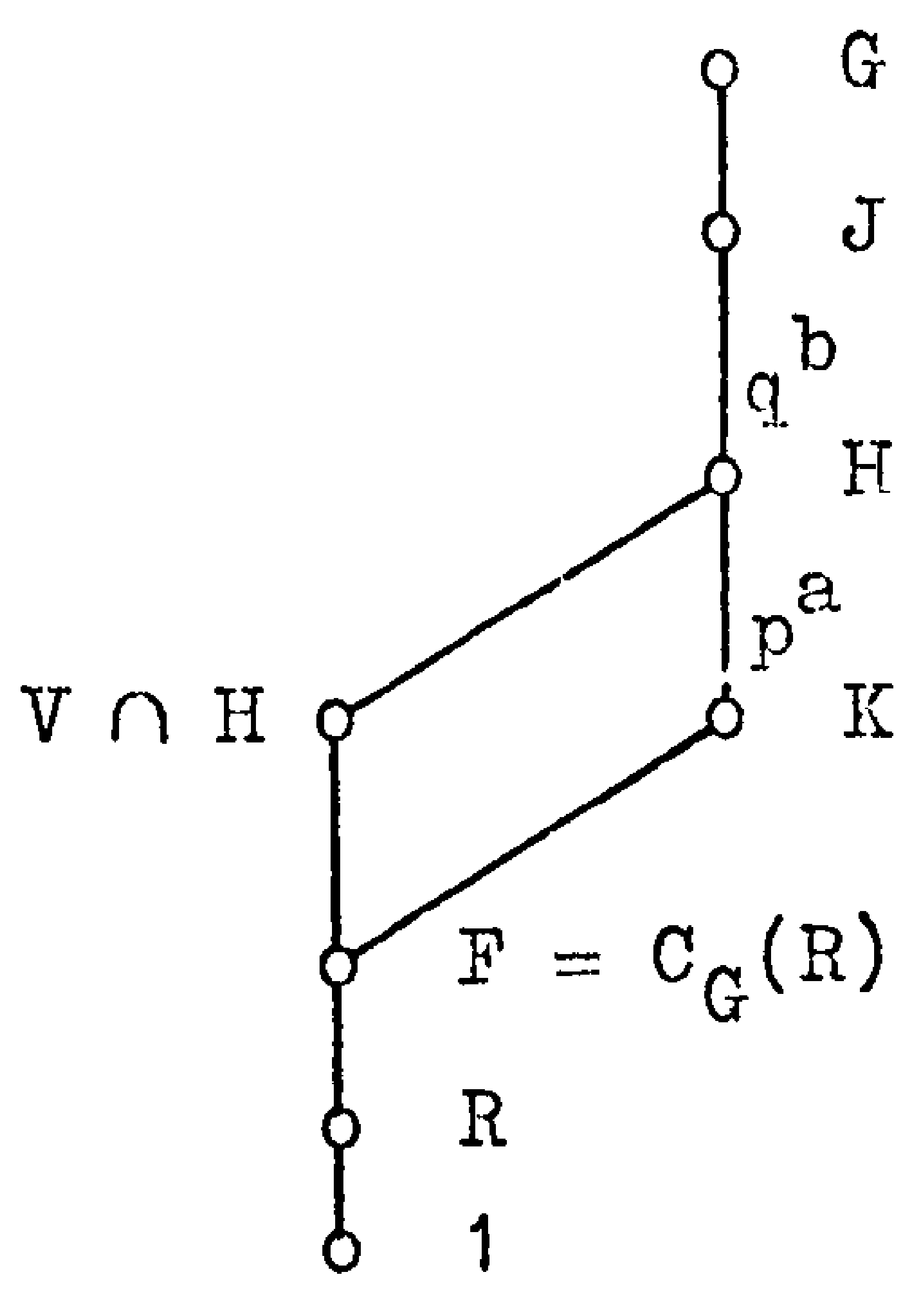
that $G / K$ is a primitive group, and so $\mathrm{J} / \mathrm{H}$ is a $\mathrm{q}$-group for some prime $\mathrm{q} \neq \mathrm{p}$. Let $V_{O}=V \cap H$ and $E_{O}=E \cap J$, so $E_{0}$ covers $J / H$ by (2). $F$ centralizes $R$, so by Naschke's theorem $O_{p^{\prime}}(R)$ is completely reducible under the action of $v_{0}$. Since $v_{0} \in \mathcal{F}_{\pi}$ it follows that $V_{0}$ centralizes $O_{p}(R)$.

Similarly $E_{O}$ centralizes $O_{q^{\prime}}(R)$. Therefore $N=C_{G}\left(O_{q^{\prime}}(R)\right)$ i.s a normal subgroup of $G$ containing $F$, which covers $\mathrm{J} / \mathrm{H}$. By (3) it follows that $H \leqslant I M$, and the Dedekind law gives $\mathrm{H}=\mathrm{K}(\mathrm{I} \cap \mathrm{H})$.

If $\mathrm{N} \cap \mathrm{H} \neq \mathrm{H}$, then $\mathrm{N} \cap \mathrm{H} \leqslant \mathrm{K}$ by (1), which contradicts (5). Thus $H \leqslant N$ which implies that $V_{0}$ centralizes $O_{q},(R)$. Then (4) and the fact that $p \neq q$ show that $V_{0}$ centralizes $R$, which is a final contradiction, showing that no such $G$ exists and the theorem follows. 


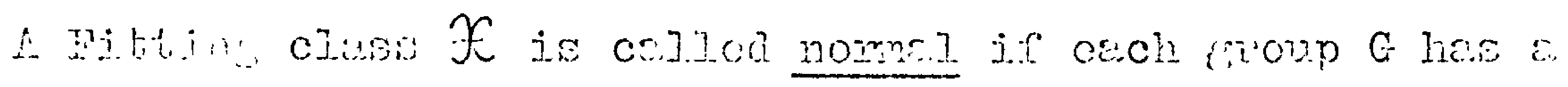
nome. (ans therefore by conjug unirue) $\mathcal{X}$-injector, conivalently, if $C_{x}$ is $\mathcal{X}$-moxims in $G$ for als. G. Such clisses cro studice by Blossonond and Goschutz in [8] , where some exmmles cre prosented. Lis the anthoms renerk, the birarre notwe of therse examples soms to indiccte that an easy clessificetion of normel fittine clesses is inprobable.

However, we maj add to our arsenal :

2.1.1 THEORtII (Blessenohl. and Gaschütz [8] Satz 3.2)

$\{G$ : each clement of $G$ induces (by conjucation) an even permutation of set of elements of $\left.\mathrm{O}_{2},(\mathrm{C})\right\}$ is a normal fitting closs.

\subsubsection{Tronm (Blessenohl and Gaschütz [8] from Satz 3.3)} Tet $\mathrm{p}$ be a prime and $\mathrm{G}$ a groun. Let $\mathrm{N}_{1}, \ldots, \mathrm{M}_{\mathrm{r}}$ be the $\mathrm{p}$-chief facturs in a chief scries of $G$, and $D_{i}: G \rightarrow G L\left(\mathbb{M}_{i}\right)$ the "cpresentation afforded by $\mathrm{H}_{i}$. By the Jordan- Holder theorem the $\operatorname{riap}_{\mathrm{G}}: \varepsilon \rightarrow \prod_{i=1}^{r} \operatorname{det}\left(D_{i}(\tilde{E})\right) \in G r(p)$, is well-defined. Then $\left\{G: d_{G}(g)=1\right.$ for all $\left.g \in G\right\}$ is a normal Fitting class. We shall require the following main result of [8] in Chapter 5.

\subsubsection{TESORTi (Blessenohl and Gaschitz [8] Satz 5.3)} $\mathcal{X}$ is a non-trivial normal Fitting class iff $G=G_{X}$ for all $G$. 2.4.4 Remerlss

a) the substance of 2.4 .3 is the implication $\Rightarrow$, the converse being clear.

b) An easy argucment of Cossey shows that cach non-trivial nomal Fituing class corstains $\mathcal{l}$, reinforcing the indication 


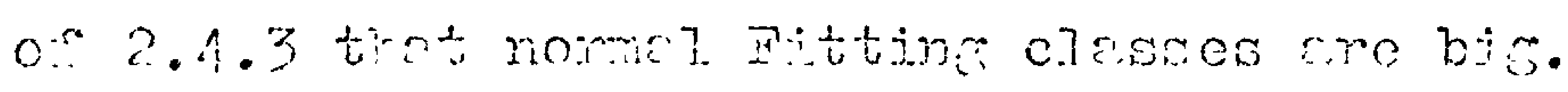

c) 2.4.3 shows that the intonoction of an arbitiong collcction

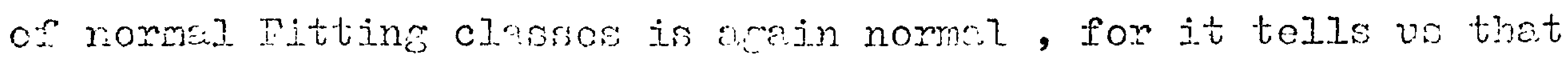
tre intericction contains $\{G: G \in S\}=\prod$ sar. In psiviculor there is a unique smilest nomel Fittins class. Jobody has as jet solved the tantalizing problem of identifying this cless, though it is not difficult to verify that it is the iritting class generoted by $\mathcal{D}$. Harres has pointed out that it dises not actually coincide with $\mathcal{D}$, since $\mathcal{D}$ fails to contain $D_{8}$. For if $D_{8} \cong G$ for some $G$, then. the fact that Aut $\left(D_{8}\right)$ is a 2-rroup inglies that we mey assume $G$ is a 2-group. But this contradicts an old result of Burnside rhich asserts that a non-1 belian Eroup of order $p^{3}$ is not the derived group of a p-iroup.

d) 1.2 .5 b) and 2.4 .3 show immediately that, for any Fitting cless $\mathcal{F}$ and normal Fitting class $\mathcal{X}$, both $\mathcal{F} \mathcal{X}$ and $\mathcal{X} \mathcal{F}$ arc nommal. Arother consequence worth remembering is that if the Fitting olass $\mathcal{F}$ contains a normal Fitting class $\mathcal{X}$, then $\mathcal{F}$ too is normal.

\subsection{Fitting formations}

Nany of the easy examples of Fitting classes are formations too. Naturally such classes are called Fitting formations. $n, S_{\pi^{\prime}}, S_{p^{\prime}} S_{p} S_{p^{\prime}}=\{$ groups of $p$-lengtin 1$\}$ are examples. Notable among those formations which are not Fitting classes are the class $O$ and the class of supersoiuble groups. This is exhibited by the groups $Q_{8}$ and a primitive split extension of $\mathrm{C}_{5} \times \mathrm{C}_{5}$ by $\mathrm{Q}_{8}$, respectively. It is well-knom (proved for example in [2]) that 3 saturated formation locally defined by Fitting formations, is again a Fitting formation. 
Thet Fittine formetions reed not be seturatod wo deronotrated

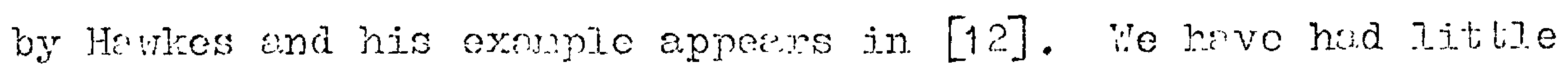
success in annlyaing, in the manner of previous results, tho behaviour of the class he defines, thongh ve focl a succossel. invertigetion would be illuminatins.

This is a convenicnt place to set out a vell-known result which we shall need later.

\subsubsection{Proposition}

If $X$ is a Fitting formation and $\mathbb{N}_{1}, \mathbb{N}_{2} \triangleleft G=\mathbb{N}_{1} N_{2}$ then $\mathrm{G}^{\mathfrak{X}}=\mathrm{N}_{1}^{\mathfrak{X}} \mathrm{IV}_{2} \mathfrak{X}$. (Here $\mathrm{G}^{\mathfrak{X}}$ denotes the $\mathfrak{X}$-residual of $G$, that j.s the unique minimal member of the set of $N \triangleleft G$ with $G / N \in X$ )

\section{Proof}

$N_{1} /\left(N_{1} \cap G^{x}\right) \cong N_{1} G^{x} / G^{x} \in S_{n}\left\{G / G^{x}\right\}$ so $N_{1}^{x} \leqslant G^{x} \cap N_{1}$ since $X=s_{n} X$. $\mathrm{G} / \mathrm{N}_{1}^{\mathfrak{X}} \mathrm{NN}_{2}^{\mathfrak{X}}=\mathrm{N}_{1} \mathrm{~N}_{2} / \mathrm{N}_{1}^{\mathfrak{X}} \mathrm{N}_{2}^{\mathfrak{X}}=\mathrm{N}_{1} \mathrm{~N}_{2}^{\mathfrak{X}} / \mathrm{N}_{1}^{\mathfrak{X}} \mathrm{NN}_{2}^{\mathfrak{X}} \cdot \mathrm{N}_{1}^{\mathfrak{X}} \mathrm{N}_{2} / \mathrm{N}_{1}^{\mathfrak{X}} \mathrm{N}_{2}^{\mathfrak{X}}$ and

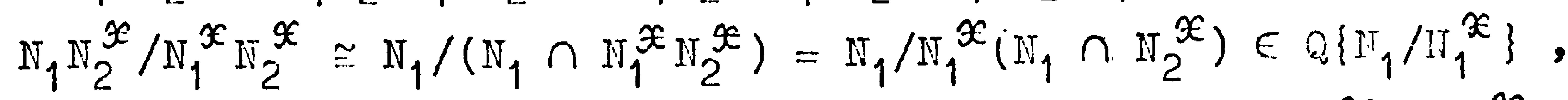
hence $\mathrm{G} / \mathrm{N}_{1}^{\mathscr{X}} \mathbb{N}_{2}^{\Re}$ is a nolmal product of $X$-groups sinco $X=\mathrm{Q} X$. Therefore $G^{\mathscr{X}} \leqslant \mathbb{N}_{1} \mathrm{~N}_{2} \mathscr{X}$ since $\mathcal{X}=\mathbb{N}_{0} \mathcal{X}$. The tro inequalities together yield the rosult.

\subsection{The classes $\mathcal{F}^{\pi}, \mathcal{F}_{1} \mathcal{F}_{2}$ and $\mathcal{F}_{1} \cap \mathcal{F}_{2}$}

If $\mathcal{F}$ :is a Fitting class and $\pi$ is a set of primes we define $\mathcal{F}^{\pi}$ to be the class of groups $G$ iri which an $\mathcal{F}$-injector of $G$ contains a Hall $\pi$-subgroup of $G$. This is clearly equivalent to the requirement that an $\mathcal{F}$-injector of $G$ covers all the $\pi$-chief factors of $G$. We maks the obvious convention that $\mathcal{F}^{\phi}=\mathcal{S}$ for all $\mathcal{F}$. Our chosen notation is perhaps poor, but has the useful advantage of brevity.

\subsubsection{Proposition}

For each $\mathcal{F}$ and $\pi$ : a) $\mathcal{F}^{\pi}$ is a Fitting class 
в) $\mathcal{F}, S_{\pi^{\prime}} \leq \mathcal{F} S_{\pi^{\prime}} \leq \mathcal{F}^{7}=\mathcal{F}^{\pi} S_{\pi^{\prime}}=\left(\mathcal{F}^{\pi}\right)^{\pi}$

c) the following are emivinent :

i.) $\mathcal{F}=\mathcal{F} S_{\pi^{\prime}}$

ii) For each $G$, en $\mathcal{F}$-injector or $G$ hess $\pi$-indez in $G$.

iii) $\mathcal{F}^{\pi^{\prime}}=S$

iv) $\mathcal{F}=\mathcal{F}^{\pi}$

a) If $\mathcal{F}$ is a fischex class, so alno is $\mathcal{F}^{\pi}$.

Proof

a) Suppose $\mathbb{V} \triangleleft G \in \mathcal{F}^{\pi}$. so let $V$ be an $\mathcal{F}$-injector or $G$ conta.ining the Hall $\pi$-subgroup $G \pi$ of $G$. Then $V \cap \mathbb{N}$ i.s an $\mathcal{F}$-injector of is containing the $H$.]. $\pi$-subgroup $G_{\pi} \cap$ N of $N$. Therefore $\mathbb{N} \in \mathcal{F}^{\pi}$.

Now suppose $\mathcal{F}^{\pi} \ni \mathrm{H}_{1}, \mathrm{~N}_{2} \triangleleft G=\mathrm{N}_{1} \mathrm{~N}_{2}$. Let $\mathrm{V}$ be an $\mathcal{F}$-injector of $G$ with a Fill $\pi$-subgroup $V_{\pi}$. Whon for $i=1,2, V_{\pi} \cap\left(V \cap N_{i}\right)$ $=V_{\pi} \cap \mathbb{N}_{i}$ is a Hall $\pi$-subgroup of the $\mathcal{F}$-injector $V \cap N_{j}$ of $\mathrm{N}_{i}$. Hov $T \in \mathcal{F}^{\pi}$ clearly imp].jes that esch Hall $\pi$-subgroup of any $\mathcal{F}$-injector of T. is a Hall $\pi$-subgroun of T. Thus $T_{\pi}$ contains Hall $\pi$-subgroups of $\mathbb{N}_{1}$ and $\mathrm{H}_{2}$ so it follows from 1.2.7 thet $V_{\pi}$ is a Hall $\pi$-suberoup of $G$ and therciore $G \in \mathcal{F}^{\pi}$.

b) The inclusions $\mathcal{F}, \rho_{\pi^{\prime}} \subseteq \mathcal{F} \rho_{\pi^{\prime}} \subseteq \mathcal{F}^{\pi} \subseteq \mathcal{F}^{\pi} \rho_{\pi^{\prime}}$ are clear. If $G \in \mathcal{F}^{\pi} S_{\pi^{\prime}}$ then an $\mathcal{F}$-injector of $G_{\mathcal{F} \pi}$ contains a Hall. $\pi$-subgroup of $G_{\text {F }}$ which is of course a Hall $\pi$-subgroup of $G$, and it fojlows that $G \in \mathcal{F}^{\pi}$.

$\mathcal{F}^{\pi} \subseteq\left(\mathcal{F}^{\pi}\right)^{\pi}$ is clear, so it remains to prove the reverse inclusion Suppose $G \in\left(\mathcal{F}^{\pi}\right)^{\pi}$ and inductively that $\left(\mathcal{F}^{\pi}\right)^{\pi}$-groups of order less than $|G|$ lie in $\mathcal{F}^{\pi}$. So a maximal nomal subgroup $\mathbb{N}$ of $G$ lies in $\mathcal{F}^{\pi}$. If $|G: N|=p \notin \pi$ then $G \in \mathcal{F}^{\pi} S_{\pi^{\prime}}=\mathcal{F}^{\pi}$ as above and we are done. If $p \in \pi$ then since $G \in\left(\mathcal{F}^{\pi}\right)^{\pi}, G / \mathbb{N}$ is covercd by an $\mathcal{F}^{\pi}$-injector of $G$, which already contains $\mathrm{N}$ so $G \in \mathcal{F}^{\pi}$ in this case too. Certainly $1 \in \mathcal{F}^{\pi}$ and the induction i.s complete so we have $\left(\mathcal{F}^{\pi}\right)^{\pi} \subseteq \mathcal{F}^{\pi}$ as required. 
c) i) $\Rightarrow$ i.i) sunnowe $\mathcal{F}-\mathcal{F} \mathcal{S}_{\pi^{\prime}}$, gis in irbitrory moun , and inductively that on F-injection of a group a of ordor loss then

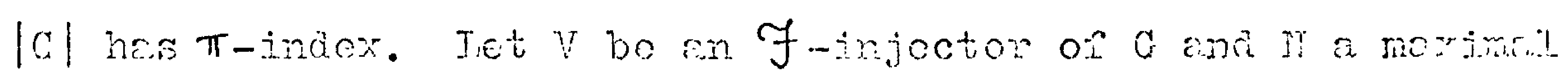
nomal subgrovp of $a$ of index $p$ say. Ey the induction bypotiosiss $V \cap \mathbb{T}$ has $\pi$-index in $T$. Thus if $p \subset \pi$ j.t follows thet $V$ has $\pi$-index in $G$ as required. Sssume $p \in \pi^{\prime}$ then by 1.1 .7 b) (a Frattini arepment) it follows that ( $T \cap M)$ is nomolizod by a HalI $\pi^{\prime}$-suberoup $G_{\pi^{\prime}}$, say, of $G$, and $V \cap I<(V \cap I) G_{\pi^{\prime}}=I$ say. By supposition $\mathcal{F}=\mathcal{F} \mathcal{S}_{\pi^{\prime}}$ so $N \in \mathcal{F}$. Wor H must be $\mathcal{F}$-mozinal in $G$, for otherwise $V \cap \mathbb{N}<H \cap \mathbb{N} \in \mathcal{F}$, contrary to the fect that $V \cap \mathbb{N}$ is an $\mathcal{F}$-injector of $N$. So by 1.1 .3 a), $H$ is an F-injector of G. H certainly hes $\pi$-index in $G$ and so the induction stie holds. The statenent is trivially true when $\mathrm{G} \cong 1$ so the proof is complete.

i.i) $\Leftrightarrow i i i)$ is cloar

ii) $\Rightarrow i v)$. Cortainly $\mathcal{F} \subseteq \mathcal{F}^{\pi}$. If $G \in \mathcal{F}^{\pi}$ then an $\mathcal{F}_{\text {-injector }}$ of $G$ has $\pi^{\prime}$-index, which with $\left.i i\right)$ implies $G \in \mathcal{F}$. iv) $\Rightarrow$ i) follows from b) immediatcly.

d) Suppose $\mathbb{N} \varangle G \in \mathcal{F}^{\pi}, \mathbb{N} \leqslant H \leqslant G$ where $H / \mathbb{N}$ is a p-iroup. We must show $H \in \mathcal{F}^{\pi}$. First assume $p \Varangle \pi$ and let $T$ be an 7 -injector of $\mathrm{H}$. $N \in \mathcal{F}^{\pi}$, so $\mathrm{T} \cap \mathbb{N}$ contains a Hall $\pi$-subgroup of $\mathbb{N}$, which by our assumption is a Hell $\pi$-subgroup of $H$. Thereforc $H \in \mathcal{F}^{\pi}$. Now suppose $p \in \pi$, and let $\mathrm{H}_{\pi}$ be a Hall $\pi$-subgroup of $\mathrm{H}$. Since $G \in \mathcal{F}^{\pi}$ we may choose an $\mathcal{F}$-injector $V$ of $G$ which contains $H$. Then $V \cap N \triangleleft V \in \mathcal{F}, V \cap N \leqslant V \cap H$ and $V \cap H / V \cap N$ is a p-Eroup so $\mathrm{V} \cap \mathrm{H} \in \mathcal{F}$ since $\mathcal{F}$ is a Fischer class. Now $\mathrm{V} \cap \mathrm{H}$ contains $H_{\pi}$ which covers $H / N \in S_{p}$, and $V \cap N$ is an $\mathcal{F}$-injector of $N$ so it follows that $\mathrm{V} \cap \mathrm{H}$ is an $\mathcal{F}$-maximal subgroup of $\mathrm{H}$. Then by 1.1 .4 a) $V \cap H$ is an $\mathcal{F}$-injector of $H$, and hence $H \in \mathcal{F}^{\pi}$. 
a) The inclusion $78_{\pi^{\prime}} \leq \mathcal{F}^{\pi}$ can be stujet, for cxemple $\Sigma_{4} \in n^{\{2\}}-\eta S_{21}$

b) whe opjorition $7 \rightarrow \mathcal{F}^{\pi}$ is no respocter of closure operations. For instence $\Sigma_{3}$ is both s quotient and $a$ suberoup of $\left.\Sigma_{4} \in \chi^{\{}\right\}$, but $\Sigma_{3} \notin \eta^{\{2\}}$ so this class does not inhorit either the a- or the s-closed property of $\eta$.

c) The equivalence of $i$ ) and $i i$ ) in $2.6 .1 \mathrm{c}$ ) (first pointed out by Gaschütz), together wit: 2.6 .1 b), shows that an $\mathcal{F}^{\pi}$-irjector $H$ of an arbitrary croup $G$ hes $\pi$-index in $G$, that is $H$ contains a Hall $\pi^{\prime}$-subgroup $G_{\pi^{\prime}}$, say, of $G$. By definition of $\mathcal{F}^{\pi}$, an F-injector $T$ of $\mathrm{H}$ contiains a Fall $\pi$-subgroup of $\mathrm{H}$, so it follows by order that $H=T F_{\pi^{\prime}}$. Whus the problem of detierminins: the $\mathcal{F}^{\pi}$-irjectors of $G$ reduces to the identification of $\mathrm{I}$. (Clearly all such subgroups T are conjugate.) A natural guess is that T. is actually an $\mathcal{F}$-injector of $G$ and this is indeed the case when $\mathcal{F}$ is a Fischer class, as we shall see in 4.1 . Unfortunately this is not alvays true and in the same section we shall present a group $G$ with an $\mathcal{F}$-injector which permutes with no Hall $\pi^{\prime}$-subgroup of $G$. In preparation we conclude this part of our discussion of $\mathcal{F}^{\pi}$ with :

\subsubsection{Proposition}

If $\mathcal{F}$ is a Fitting class and $\pi$ a set of primes, and an F-injector $V$ of $G$ permutes with a Hall $\pi^{\prime}$-subgroup $G_{\pi^{\prime}}$ of $G$, then $\mathrm{H}=\mathrm{VG}_{\pi^{\prime}}$ is an $\mathcal{7}^{\pi}$-injector of $\mathrm{G}$.

Proof

The proposition is trivially true if $|G|=1$, so we use induction, supposing the result true for groups of order less than $|G|$. Iet $N$ be a maximal normal subgroup of $G$. By order, a Hall $\pi$-subgroup $V_{\pi}$ of $V$ is a Hall $\pi$-subgroup of $H$, so $H=$ $V_{\pi} G_{\pi}$. It follows that $\left(V_{\pi} \cap N\right)$ and $\left(G_{\pi}, \cap N\right)$ are Hall subgroups 


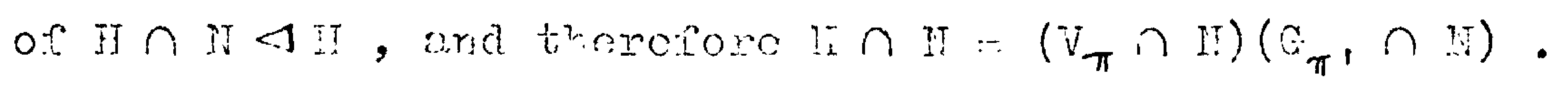

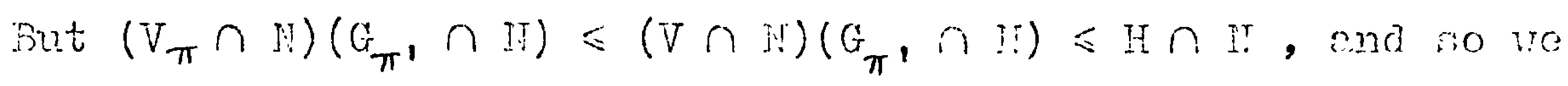
hovo equality throvenout. $V \cap \|$ and $C_{\pi}, \cap \mathrm{N}$ s.re en $\mathcal{F}$-injector and a. Hall $\pi^{\prime}$-subrroup of II resnectively, so hy induction $H \cap \mathbb{N}$ is an $\mathcal{F}^{\pi}$-injector of $\mathrm{H}$. We now shov If is $\mathcal{F}^{\pi}$-maximal in $G$, then by 1.1 .4 a) i.t follows thet $H$ is en $\mathcal{F}^{\pi}$-injector of $G$ completing the induction argupment. So sup rose if $\leqslant K$ where $K$ is $\mathcal{F}^{\pi}$-maximal in $G$. $K$ contains $V$ and $G_{\pi^{\prime}}$ and by $\left.1.1 .4 \mathrm{c}\right) \mathrm{V}$ is an $\mathcal{F}$-injector of $K$. Therefore $V$ contains a Hell $\pi$-suberoup of $\mathrm{K}$ and hence $\mathrm{K}=\mathrm{VG}$, by order, yhich yields $\mathrm{K}=\mathrm{H}$.

The following cheracterizetion of the injectors for the Fitting class $\mathcal{F}_{1} \mathcal{F}_{2}$ is an application of our $\mathcal{F}^{\pi}$ concept.

\section{$\frac{2.6 .4 \quad \text { THOREM }}{4-7}$}

Let $\mathcal{F}_{1}$ and $\mathcal{F}_{2}$ be Fitting clesses and $G$ a group. Put $\mathcal{F}=\mathcal{F}_{1} \mathcal{F}_{2}$ and $\pi_{2}=\operatorname{char} \mathcal{F}_{2}$. Let $T$ be an $\mathcal{F}_{1}$-injector of $G_{\mathcal{F}_{1}} \pi_{2}$. By definition of $\mathcal{F}_{1} \pi_{2}$ and the Frattini argudment, $T$ is normalized by some Hall $\pi_{2}$-subsroup $G_{\pi_{2}}$ of $G$. Let $V / T$ bc an $\mathcal{F}_{2}$-injector of $\mathrm{TG}_{\pi_{2}} / \mathrm{T}$, then $\mathrm{V}$ is an $\mathcal{F}$-injecior of $G$. PROOF

We first:establish the result in two special cases.

(1) If $G \in \mathcal{F}_{1} \pi_{2}$, then an $\mathcal{F}_{1}$-injector of $G$ is an $\mathcal{F}_{\text {-injector }}$ of $G$.

This is trivial if $G=1$, so we areue hy induction, supposing (1) holds for groups of order less than $|G|$. Let $\mathbb{I}$ be a maximal normal subgroup of $\mathrm{G}$ of index $\mathrm{p}$ say, and $\mathrm{H}$ an $\mathcal{F}_{1}$-injector of G. Certainly $\mathbb{N} \in \mathcal{F}_{1} \pi_{2}$. If $\mathrm{H}$ covers $\mathrm{G} / \mathbb{N}$, (as is of course the case when $\mathrm{p} \in \pi_{2}$ since $\mathrm{G} \in \mathcal{F}_{1} \pi_{2}$ by assumption) then $\mathrm{H} \in \mathcal{F}$ and $H$ properly contains $H \cap N$, which by induction is an 


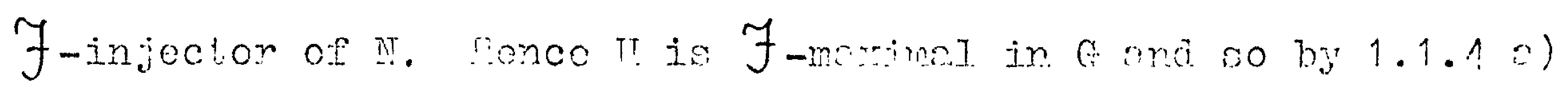
is $\sin 7-j r j o c t o n$ or $a$.

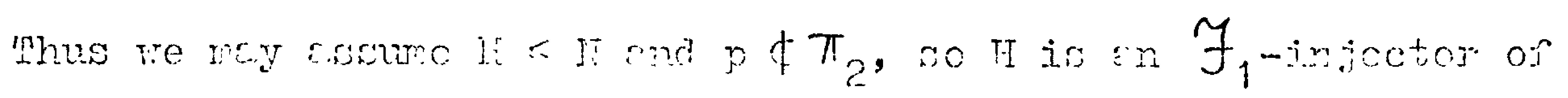
$\mathrm{N}$ and thorefore by induction iso on $\mathcal{F}$-injector of $\mathrm{N}$. But then if $I<J \in \mathcal{F}$, it followa that $H=J \cap I$ and so $I I=J_{\mathcal{F}}$ because $I$ is $\mathcal{F}_{1}-\operatorname{mrimal}$ in $G$. Whon $J / J \cap I \cong G / \mathrm{I} \cong \mathrm{C}_{\mathrm{p}}$ \& $\mathcal{F}_{2}$ which contradicts $J \subset \mathcal{F}$. ie deduce that $\mathrm{H}$ is $\mathcal{F}$-moximal in $G$ and by 1.1 .4 a) arain, $H$ is an 7 -injoctor or $G$.

(2) If $G \in \mathcal{f}_{1} \pi_{2} \eta$, thon $\mathrm{C}_{\pi_{2}}$ is an $\mathcal{F}$-injector of $G$. since $\mathcal{F}_{1} \pi_{2}=\mathcal{F}_{1} \pi_{2} \mathcal{S}_{\pi_{2}}$, by $\left.2.6 .1 \mathrm{~b}\right), G \in \mathcal{F}_{1} \pi_{2} \eta_{\text {imnlies }}$ $\mathrm{G} / \mathrm{G}_{\mathcal{F}_{1} \pi_{2}} \in \Re_{\pi_{2}}$, and $\eta_{\pi_{2}} \subseteq \mathcal{F}_{2}$ by definition of $\pi_{2}$. Therefore $T G_{\pi_{2}}$ lies in $\mathcal{F}$ and covers $G / G_{\mathcal{F}_{1}} \pi_{?}$. (2) then follows from (1) and $1.1 .4 \mathrm{~b})$

Now let $K=F\left(G \bmod G_{\mathcal{F}_{1} \pi_{2}}\right)=G_{\eta_{1}} \pi_{2} \eta^{\circ}$ Put $I_{\pi_{2}}=K \cap G_{\pi_{2}}$, a HaII $\pi_{2}$-subgroup of $K$. By (2) $\mathrm{Tr}_{\pi_{2}}$ is an $\mathcal{F}$-injector of $K$. (3) $\mathrm{T}$ is $\mathcal{F}_{1}$-maximal in $\mathrm{K}$.

For if not suppose $\mathrm{T}<\mathrm{T}^{*} \leqslant K$ with $\mathrm{T}^{*} \in \mathcal{F}_{1}$. Then by $1.1 .4 \mathrm{a}$ ) $\mathrm{T}^{*}$ is an $\mathcal{F}_{1}$-injector of $\mathrm{G}_{\mathcal{F}_{1}} \pi_{2} \mathrm{~T}^{*}=\mathrm{S}$ say. It follows that $S \in \mathcal{F}_{1}^{\pi_{2}}$, but $S$ an $K \Delta G$, so that $S \leqslant G \mathcal{F}_{1} \pi_{2}$, which implies $T^{*} \leqslant G_{\mathcal{F}_{1}} \pi_{2}$, a contradiction to the fact that $\mathrm{T}$ is an $\mathcal{F}_{1}$-injector of $G_{1} \pi_{2}$

(4) Suppose either a) $\mathrm{TK}_{\pi_{2}} \leqslant H \in \mathcal{F}$

$$
\text { or b) } M K_{\pi_{2}} \leqslant H \leqslant T G_{\pi_{2}} \text {, then } H_{\mathcal{F}_{1}}=T \text {. }
$$

We have $T=E \cap G_{\mathcal{F}_{1}} \pi_{2} \triangleleft H$ and $T K_{\pi_{2}}=H \cap K \triangleleft H$, beceuse 


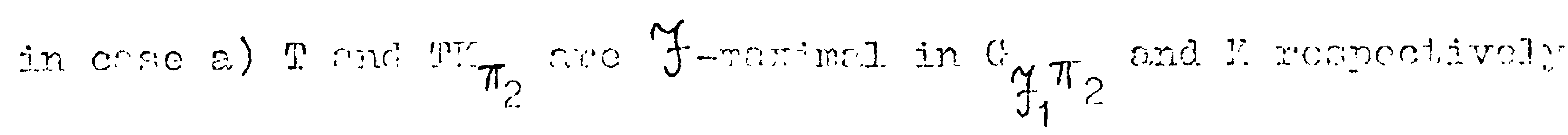

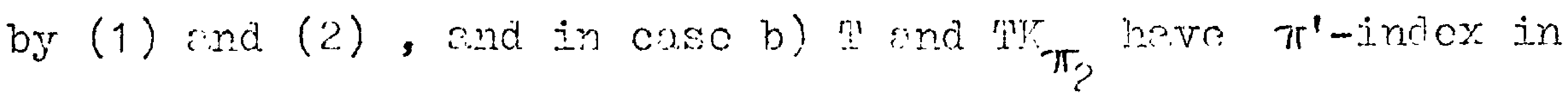

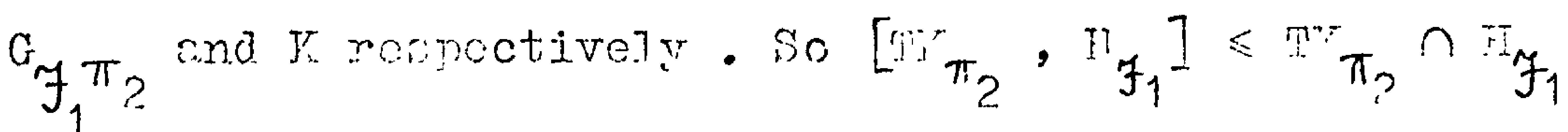
$K \cap I_{F_{1}} \in \mathcal{F}_{1}$. cortainly $T \leqslant Y \cap F_{F_{1}}$, since $T$ is s nome.

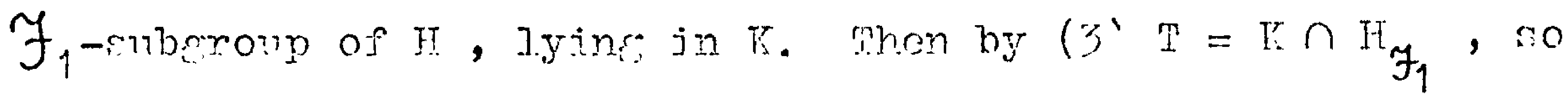
we heve $\left[I_{\pi_{2}}, I_{F_{1}}\right] \leqslant \mathrm{T}$ and thereforc $\left[G_{F_{1}} \pi_{2}, \mathrm{H}_{\mathcal{F}_{1}}\right] \leqslant \mathrm{G}_{\mathcal{F}_{1}} \pi_{2}$ which seys $\mathrm{H}_{\mathcal{F}_{1}}$ centralires $\mathrm{F}\left(\mathrm{G} / \mathrm{G} \mathrm{F}_{1} \pi_{2}\right)$. Tor a stendard result implies $\mathrm{H}_{\mathcal{F}_{1}} \leqslant \mathrm{r}$ and so $\mathrm{T}=\mathrm{H}_{\mathcal{F}_{1}}$ as required.

(5) The canonical map induces a 1-1 correspondonce between $\left\{\mathcal{F}\right.$-suberoups of $T G_{\pi_{2}}$ containing $\left.T \pi_{2}\right\}$ and $\left\{\mathcal{F}_{2}\right.$-subroups of $\mathrm{TG}_{\pi_{2}} / \mathrm{T}$ containing $\left.\mathrm{TK}_{\pi_{2}} / \mathrm{T}\right\}$. For if $\mathrm{TK}_{\pi_{2}} \leqslant \mathrm{H} \leqslant \mathrm{TG}_{\pi_{2}}$, then by (4) $\mathrm{H}_{\mathrm{F}_{1}}=\mathrm{T}$ so that $H \in \mathcal{F}$ iff $H / T \in \mathcal{F}_{2}$.

Now let $H$ be an $\mathcal{F}$-injector of $G$. Passing to a conjugate if necessary, wo mey assume that $H$ contains $\mathrm{PK} \mathrm{T}_{2}$, since by (2) $\mathrm{TK}_{\pi_{2}}$ is an 7 -injector of the normal subcroup $\mathrm{K}$ of $\mathrm{G}$. Then by (4) $\mathrm{H}_{\mathrm{F}_{1}}=\mathrm{T}$, so II/T is a $\pi_{2}$-subgroup of $I_{G}(\mathrm{~T}) / \mathrm{T}$, of which $\mathrm{IG}_{\pi_{2}} / \mathrm{T}$ is a. Inll $\pi_{2}$-suboroup. Thus we mey assume further. that $H \leqslant T G_{\pi_{2}}$.

Now let $\mathrm{T} \triangleleft \mathrm{IK}_{\pi_{2}} \triangleleft \ldots . \triangleleft \mathrm{TG}_{\pi_{2}}$ be a series with nilpotent factors. Then using $1.1 .4 \mathrm{~b}$ ) and (5) we sce that $\mathrm{H} / \mathrm{T}$ is an $\mathcal{F}_{2}$-injector of $\mathrm{TG}_{\pi_{2}} / \mathrm{T}$ and hence conjugate to the subgroup $\mathrm{V} / \mathrm{T}$ constructed in the statement of the theorem. So $V$ is an $\mathcal{F}$-injector of $G$ as asserted.

\section{6 .5 Corollary}

suppose $\mathcal{F}_{1}, \mathcal{F}_{2}, \ldots, \mathcal{F}_{n}$ are Fitting classes and put 


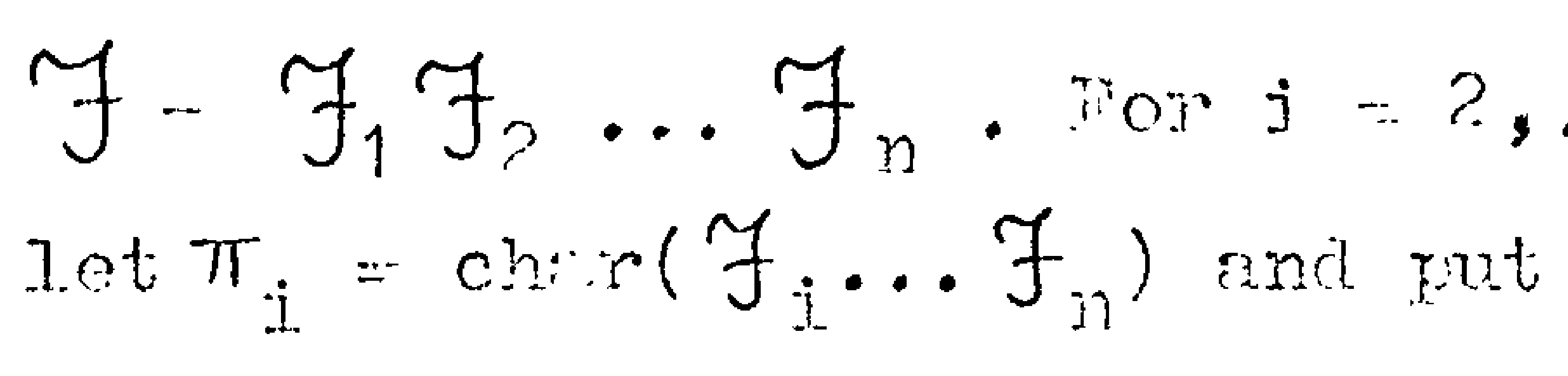

$\pi_{n+1}=\phi$. Int $c_{0}=1$ anc for

i. $\therefore 1, \ldots, n$ let $G_{i}=G_{\mathcal{F}_{1}} \pi_{2}, \mathcal{F}_{2} \pi_{3}, \ldots \mathcal{F}_{i} \pi_{i+1}$

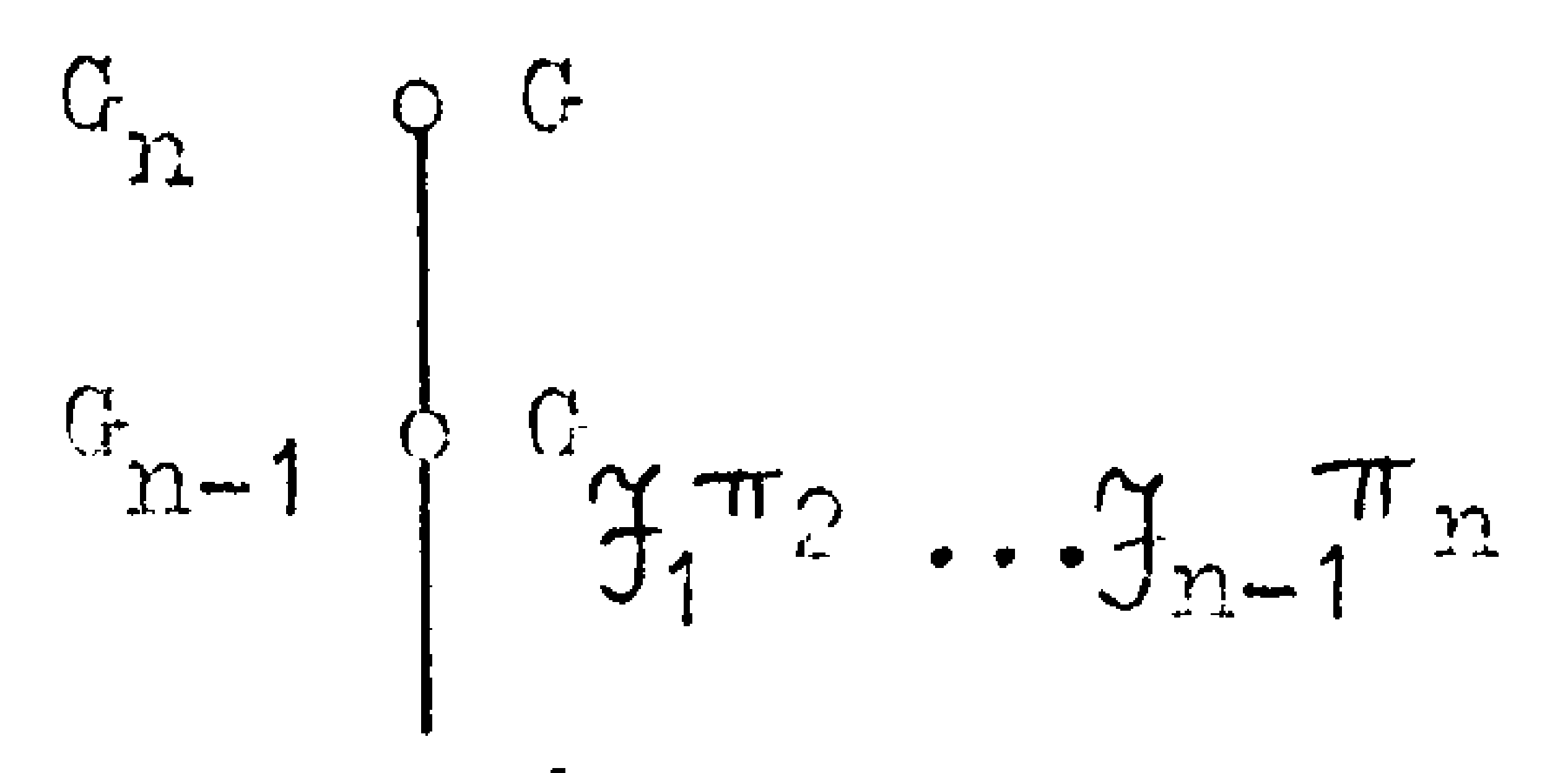

so by 1.2 .5 b) $G_{i} / G_{i-1}=\left(G / G_{i-1}\right)_{\mathcal{F}} \pi_{i+1}$

Iotice $\mathcal{F}_{n} \pi_{n+1}=S$ so $c_{n}=G$.

Suppose $V$ is an $F$-injector of $a$,

then $\left(V \cap G_{i}\right) G_{i-1} / G_{j-1}$ is an

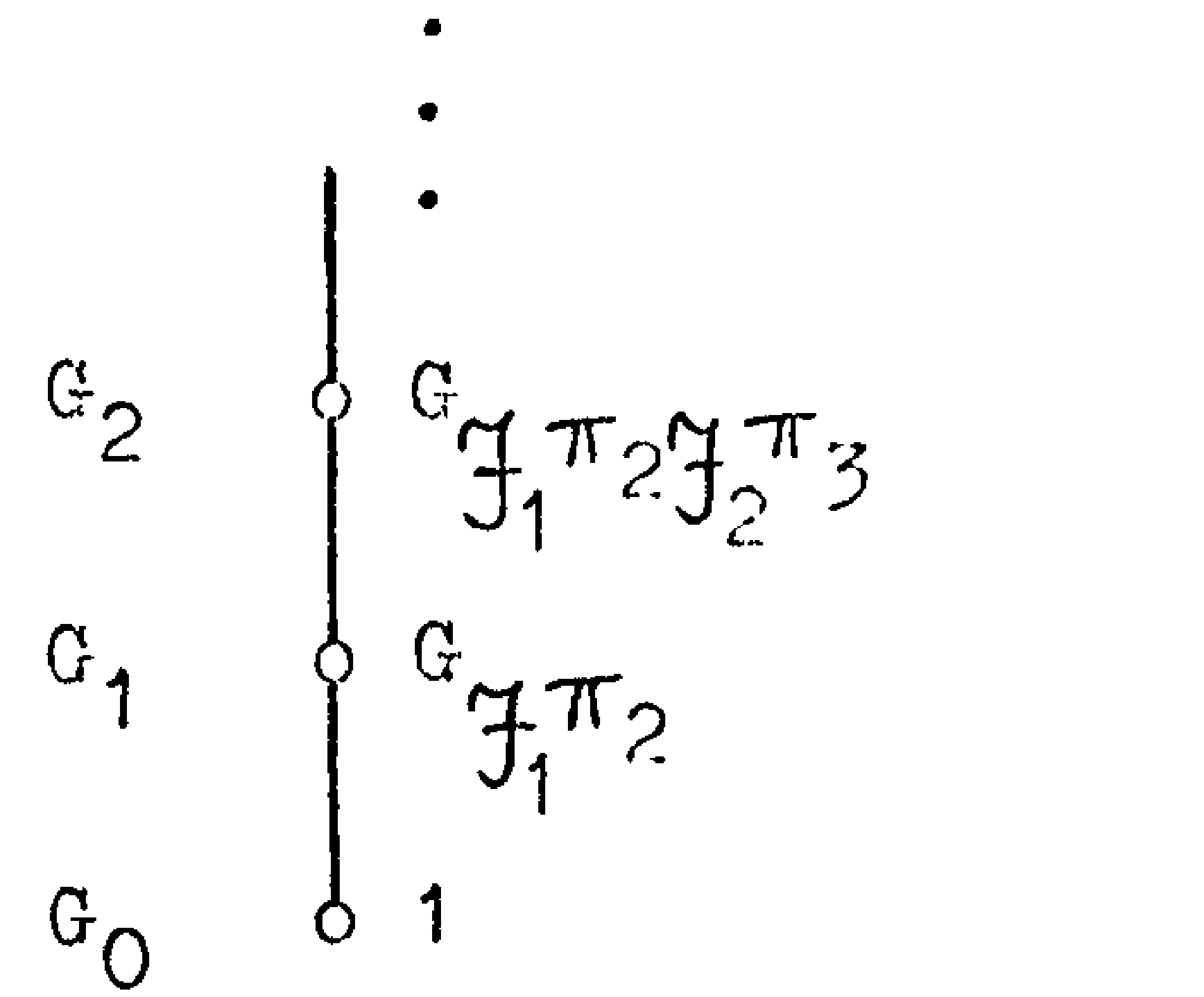

$\mathcal{F}_{j}$-injector of $G_{i} / G_{j-1}$. Tooscly

$V$ covers precisely an $\mathcal{F}_{i}$-injector of the $'$ ith $'$ layer of $G$.

\section{Proof}

The result is obvious when $n=1$.

Suppose $n=2$. By 2.6.4 and the conjugacy of injectors, $\mathrm{V} / \mathrm{T}$ is an $\mathcal{F}_{2}$-injector of $\mathrm{TG}_{\pi_{2}} / \mathrm{T}$ for some $\mathcal{F}_{1}$-injector $\mathrm{T}$ of $\mathrm{G}_{1}$ and $\mathrm{Iall} \pi_{2}$-subcroup $G_{\pi_{2}}$ of $G$. Now for $x \in \pi_{\pi_{2}}$, the map $T x \rightarrow G_{1} x$ is an isomorphism $T G_{\pi_{2}} / I \cong G_{1} G_{\pi_{2}} / G_{1}$ since $T=T G_{\pi_{2}} \cap G_{1}$. Thus $\mathrm{VG}_{1} / \mathrm{G}_{1}$, the image $\mathrm{cf} \mathrm{V} / \mathrm{T}$ under this map, is an $\mathcal{F}_{2}$-injector of $G_{1} G_{\pi_{2}} / G_{1}$ which in tirn is a Hall $\pi_{2}$-subgroup of $G / G_{1}$. Since char $\mathcal{F}_{2}=\pi_{2}$, . it follows that $V G_{1} / G_{1}$ is an $\mathcal{F}_{2}$-injeotor of $G / G_{1}$ and we already know $V \cap G_{1}=T$ is an $\mathcal{F}_{1}$-injector of $G_{1}$, so tire result holds in this case.

Now suppose $n>2$ and inductively that the result holds for the product of at most $n-1$ classes. $\operatorname{set} i n g \mathcal{H}=\mathcal{F}_{2} \mathcal{F}_{3} \ldots \mathcal{F}_{n}$, the induction hypothesis implies that $V G_{1} / G_{1}$ is an $\mathcal{H}$-injector of $G / G_{1}$ and $V \cap G_{1}$ is an $F_{1}$-injector of $G_{1}$. since $G_{i} / G_{1}=$ $\left(G / G_{1}\right)_{F_{2}} \pi_{3} \ldots \mathcal{F}_{i} \pi_{i+1}$ for $i=2, \ldots, n$, a second epplication of induction (vith a suitable isomorphism theorem) gives the statement for $n$ classes and the proof is complete. 
$2.6 .5^{1}$ Corotisny

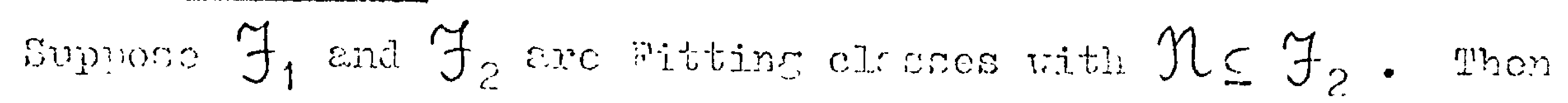
for arch $G$ a) $I_{\mathcal{F}_{1} \mathcal{F}_{2}}(G)=I_{\mathcal{F}_{2}}\left(G \bmod G_{\mathcal{F}_{1}}\right)$

arda b) $\operatorname{III}_{\mathcal{F}_{1} \mathcal{F}_{2}}(G)=\operatorname{III}_{\mathcal{F}_{2}}\left(G \bmod G_{\mathcal{F}_{1}}\right)$.

In pexticular, if also $I_{F_{2}}(G)={ }_{1} I_{F_{2}}(G)$ for all $G$ then $I_{\mathcal{F}_{1} \mathcal{F}_{2}}(G)=\operatorname{III}_{\mathcal{F}_{1} \mathcal{F}_{2}}(G)$ for $2.1 I G$.

Iroof

a) If $\chi_{\subseteq} \mathcal{F}_{2}$ then char $\mathcal{F}_{2}$ is thoset of all. prines. Cleerly $\dddot{Y}_{1}\{a I l$ priries $\}=\mathcal{F}_{1}$ so in this case 2.6 .4 states that the inverse image of an $\mathcal{F}_{2}$-injector of $G / G_{\mathcal{F}_{1}}$ in $G$, is an $\mathcal{F}_{1} \mathcal{F}_{2}$-jnjector of $G$ and by their conjugacy all $\mathcal{F}_{1} \mathcal{F}_{2}$-injectors of $G$ have this form. This is statement a)

b) Suppose $G_{\mathfrak{F}_{1} \mathfrak{F}_{2}} \leqslant H \leqslant G$. Since $G_{\mathfrak{F}_{1} \mathcal{F}_{2}} \triangleleft$ II we have $G_{\mathfrak{F}_{1} \mathfrak{F}_{2}} \cap \mathrm{H}_{\mathfrak{F}_{1}}=\left(G_{\mathfrak{F}_{1} \mathfrak{F}_{2}}\right)_{\mathfrak{F}_{1}}=G_{\mathfrak{F}_{1}}$ as usual, so $\left[G_{\mathcal{F}_{1} \mathfrak{F}_{2}}, \mathrm{H}_{\mathfrak{F}_{1}}\right] \leqslant G_{\mathcal{F}_{1}}$. We deduce that $\mathrm{H}_{\mathcal{F}_{1}}$ centralizes $G_{\mathcal{F}_{1} \mathcal{F}_{2}} / G_{\mathcal{F}_{1}}$, which by the supposition $\eta \subseteq \mathcal{F}_{2}$ contains the Fitting subgroup of $G / G_{F_{1}}$. So by the often quoted result, $\mathrm{H}_{\mathcal{F}_{1}} \leqslant \mathrm{G}_{\mathcal{F}_{1} \mathcal{F}_{2}}$ and hence $\mathrm{H}_{\mathcal{F}_{1}}=\mathrm{G}_{F_{1}}$. (The crux of the proof of 2.6.4 is a disguised form of this argulment) We have $H \in \mathcal{F}_{1} \mathcal{F}_{2}$ iff $I / G_{F_{1}} \in \mathcal{F}_{2}$ and $b$ ) is now clear.

Despite $I_{S_{\pi}}(G)=\operatorname{III} g_{\pi}(G)$ for all $G$, example 1.2 .6 shows that the final sentence of the statcment of 2.6 .5 does not follow withovt the assumption $\eta \subseteq \mathcal{F}_{2}$. (Recall that by 1.2 .2 , $\eta \nsubseteq \mathcal{F}_{2}$ and $I_{\mathcal{F}_{2}}(G)=\operatorname{III}_{\mathcal{F}_{2}}(G)$ for all $G$ implies $\mathcal{F}_{2}=S_{\pi}$ for some $\left.\pi_{0}\right)$ However I $S_{\pi^{\prime}} \beta_{\pi}(G)=\operatorname{III} S_{\pi^{\prime}} S_{\pi}(G)=\operatorname{HaII}\left(G \bmod 0_{\pi^{\prime}}(G)\right)$ holds for each $G$ by a similar proof since $0_{\pi^{\prime} \pi}(G) / 0_{\pi^{\prime}}(G)$ contains its centralizer in $G / 0_{\pi^{\prime}}(G)$ for all $G$, as is well known.

\section{$\underline{2.6 .6 \text { THEOREM }}$}

If $\mathcal{F}_{1}$ and $\mathcal{F}_{2}$ are Fischer classes then so also is $\mathcal{F}_{1} \mathcal{F}_{2}$. 


\section{PrOCOH}

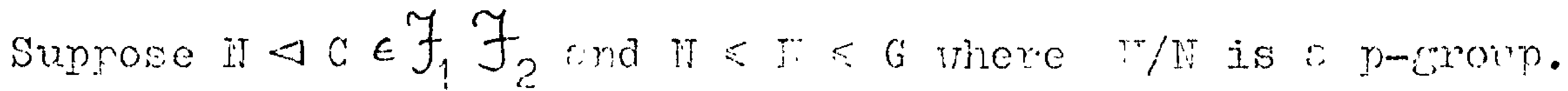
ic recuire $H \in 7_{1}, 7_{2}$.

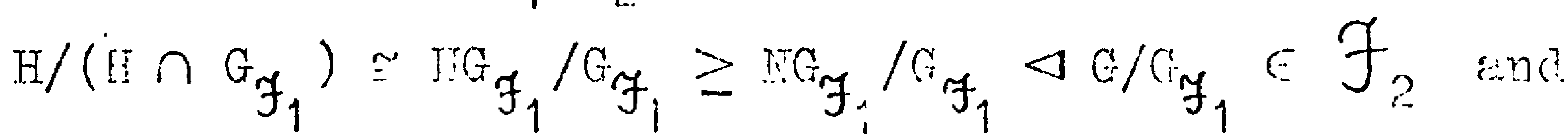

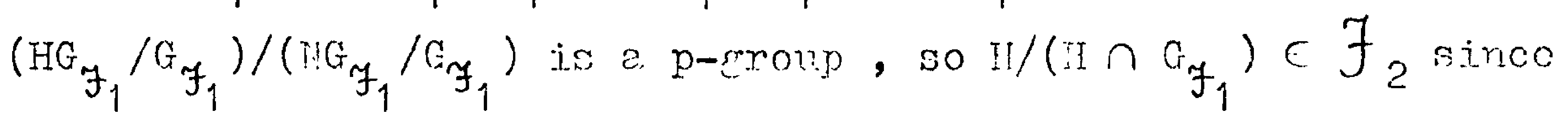
$7_{2}$ is a Fischer cless by essumption.

$H \triangleright \mathrm{H} \cap G_{\mathcal{F}_{1}} \geq I \cap G_{\mathcal{F}_{1}} \neg G_{\mathcal{F}_{1}} \in \mathcal{F}_{1}$ and $\mathrm{I} \cap G_{\mathcal{F}_{1}} / I \cap G_{\mathcal{F}_{1}}$ i.s a p-group, so II $\cap G_{f_{1}} \in \mathcal{F}_{1}$ since $\mathcal{F}_{1}$ is a Eischer class by assumption. Therefore II $\cap \mathrm{G}_{\mathcal{F}_{1}} \leqslant \mathrm{H}_{\mathcal{F}_{1}}$, and so

$H_{\mathcal{F}_{1}} \cap\left(I \cap G_{\mathcal{F}_{1}}\right) H=\left(I \cap G_{\mathcal{F}_{1}}\right)\left(H_{\mathcal{F}_{1}} \cap I T\right)=\left(I I \cap G_{\mathcal{F}_{1}}\right) H_{\mathcal{F}_{1}}=I \cap G_{\mathcal{F}_{1}}$ with help from the nodular lav. Thus ie rave:

$\left(\mathrm{H} \cap \mathrm{G}_{\mathcal{F}_{1}}\right) \mathrm{N}$

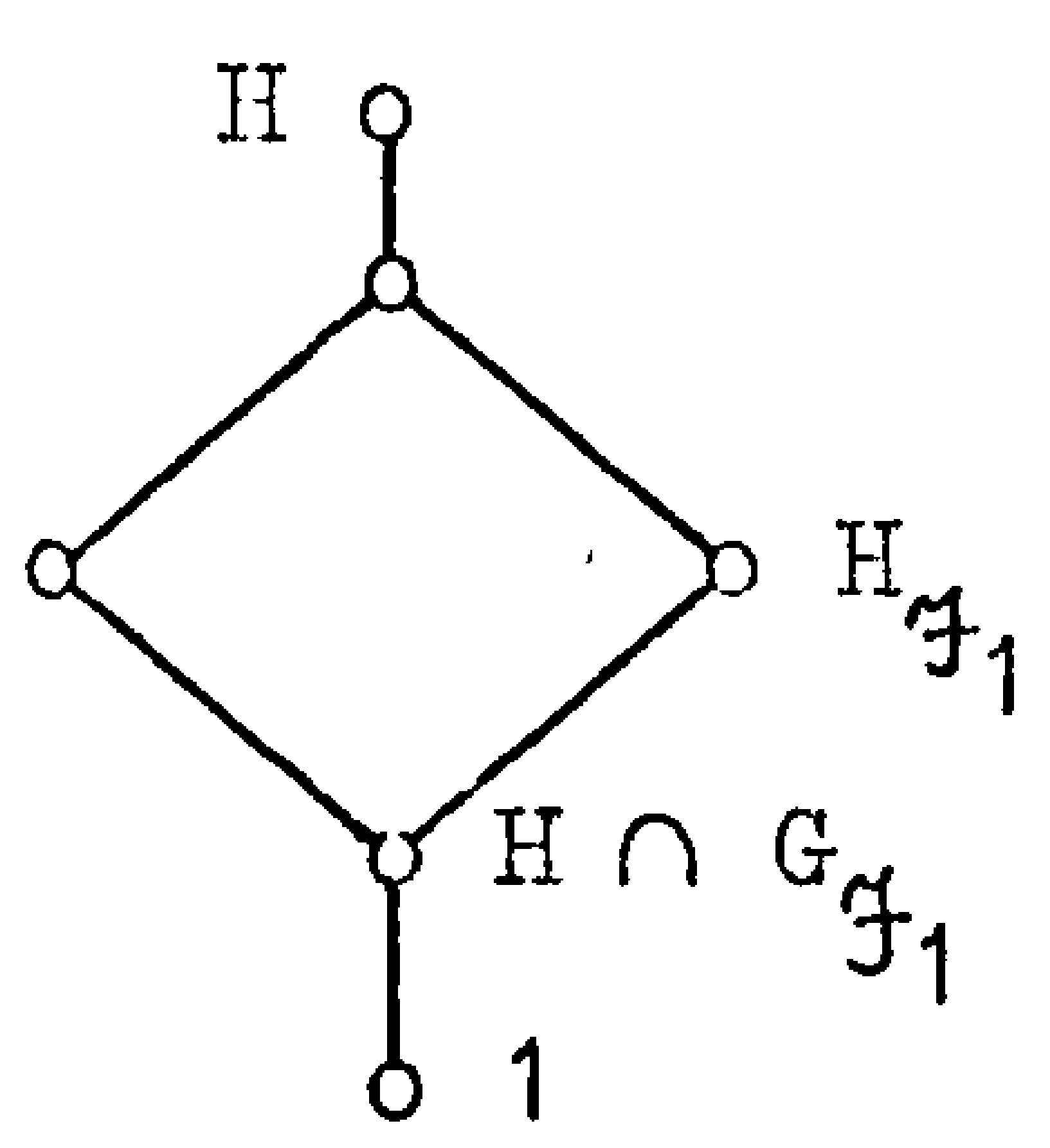
$H /\left(I \cap G_{q_{1}}\right) \in \mathcal{F}_{2}$ and $H /\left(H \cap G_{f_{1}}\right) N$ is a $p$-eroup so it too ljes in $\mathcal{F}_{2}$.

Applying part b) of the following lemma, we deducc $\mathrm{H}_{1} \mathrm{H}_{F_{1}} \in \mathcal{F}_{2}$.

\section{6 .7 Lemma}

Let $\mathcal{F}$ be a Pitting class and suppose $A, B \triangleleft G$ with $A \cap B=1$ and $G / A B \in \Re$. Then a) $G / A, G / B \in \mathcal{F}$ implies $G \in \mathcal{F}$, and b) $G / A, G \in \mathcal{F}$ impries $G / B \in \mathcal{F}$.

\section{Proof}

We need only show that $G$ is isomorphic to a subnormal subgroup $G_{O}$ of $X=G / A \times G / B$ and furthermore that $X$ is generated by $G_{O}$ and $G / \Lambda \times 1$. The conclusions then follor immediately.

Let $N=A B$ and put $G_{O}=\{(A g, B g): E \in G\}$, then $G \cong G_{0}$ since $A \cap B=1$. Also $G_{O} \geq N / A \times N / B$ and so $G_{0}$ sn $X$ because $G / N \in \Re \cdot(A, B g)=(A g, B g)(A g, B)^{-1}$ so $X=G_{0}(G / A \times 1)$, and the proof is complete. 
a) In scction 3.? of [1] Inrtiey cives the whort armefnut which wows thet for an erbitmery witine clese $F$ and set of primes $\pi, 78_{\pi} S_{\pi^{\prime}}$ j.s actuelly a wischer cless , so a nonFischer class cunnot be crpressed in the form $\bigcap_{p} f(n) S_{p} S_{p}$ for sore choice of Fitting classes $\{\mathcal{F}(p)\}$. Ilotice that $\mathcal{F} \eta=\bigcap_{\mathrm{p}} \mathcal{F} S_{\mathrm{p}} S_{\mathrm{p}}$, so this too is a Fisciser class for eny $\mathcal{F}$. T.t is not difficult to show that the rischer class $e_{q}\left(\beta_{q}\right)$, mentioned in 2.2 .3 cannot be put in this form either, so there is no hope that this direct analogue of locel defirition for saturated formations, yields even all the Fischer classes. We vorida point oul that the cless $\mathcal{F}=\bigcap_{\text {all }} F(p) \cdot S_{p}, S_{p}$, considered by Wartley in section 3.3 of his paper, actualiy coincides with $\overline{\mathcal{F}} れ$ where $\overline{\mathcal{F}}=\bigcap_{\text {all }} \mathcal{F}(p) S_{p}$, , so by 2.6 .5 $I_{\mathcal{F}}(G)=\operatorname{III}_{\mathcal{F}}(G)=I_{\mathfrak{X}}\left(G \bmod G_{\mathcal{F}}\right)$ in this case.

b) We have been unable to settle the problem :

* $\quad s \mathcal{F}_{i}=\mathcal{F}_{i}$ for $i=1,2$ implies $s \mathcal{F}_{1} \mathcal{F}_{2}=\mathcal{F}_{1} \mathcal{F}_{2}$ :

It can easily be seen that an example which exhibits the falsehood of * vould necessarily heve $\mathcal{F}_{2} \neq Q \mathcal{F}_{2}$, and conversely. it is very likely that such a class $\mathcal{F}_{2}$ could be "incorporated into an example to deny *. Nobody seems to know a Fitting class $\mathcal{F}$ with $s \mathcal{F}=\mathcal{F} \neq Q \mathcal{F}$, though we expect one exists. Since $\left\{\mathrm{S}, \mathrm{N}_{0}\right\}$-closed implies $\left\{\mathrm{N}, \mathrm{D}_{0}\right\}$-closed implies $\mathrm{n}_{0}$-closed, this means that all known S-closed Fitting ciasses are actually S-closed Fitting formations. Hawkes has conjectured that these latter classes are the so-called primitive saturated formations,

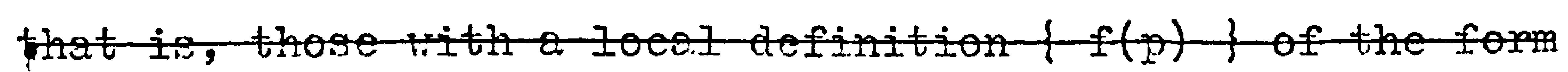
$f(p)=\frac{f_{\pi(p)}}{\text {. }}$.

c) As in 2.3 let $\mathcal{F}_{p}$ be the class of groups vith central p-socle. 
The following exorpe whow thet the $\mathcal{F} \rightarrow \mathcal{F}^{\pi}$ oprestion

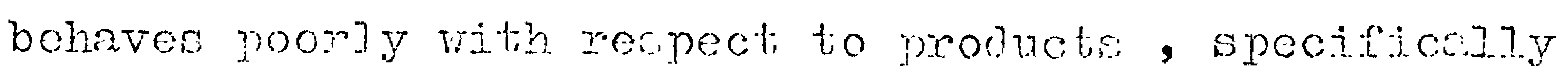

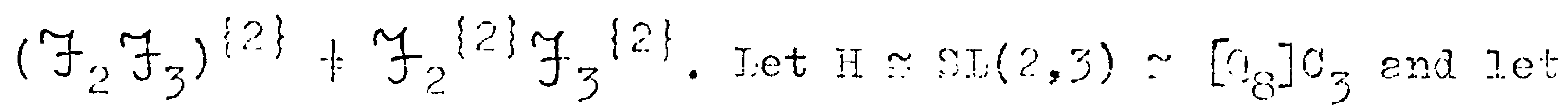
$G=H 2 \Sigma_{z}$ consinucted with tive neturi. pornutetion rerrecontation of $\Sigma_{3}$, so the base aroup $B$ of $G$ is joororphic to $\mathrm{H} \times \mathrm{H} \times \mathrm{H}$. If has a contral unique minimal normal subgroup $z$ say, of order 2 , and hence $B \in \mathcal{F}_{2} \cdot \mathrm{BC}_{3} \notin \mathcal{F}_{2}$ (where $\mathrm{C}_{3}$ is the sylow 3-subcroup of $\Sigma_{3}$ ) since $C_{3}$ acts in a non-triviel and corpletely reducible manner on $z \times z \times 2$. It follows that $13 C_{3}$ is an $\mathcal{F}_{2} \mathcal{F}_{3}$-injector cf $G$, so $G \&\left(\mathcal{F}_{2} \mathcal{F}_{3}\right)^{\{2\}}$. However $1 \mathrm{C}_{2}$ (where $c_{2}$ is a isylor 2-subgroup of $\left.\Sigma_{3}\right)$ is an $\mathcal{F}_{2}$-injector of $G$ and covers all its 2-chief factors, so $G \in \mathcal{F}_{2}\{2\}$ and honce $G \in \mathcal{F}_{2}\{2\} \mathcal{F}_{3}\{2\}$.

We end our ' new from old' section vith sone observetions on the intersection $\mathcal{F}_{1} \cap \mathcal{F}_{2}$ of two pitting classes $\mathcal{F}_{1}$ and $\mathcal{F}_{2}$. It seems rarely possible to relate the injectors of a group $G$ for the class $\mathcal{F}_{1} \cap \mathcal{F}_{2}$ to those for the classes $\mathcal{F}_{1}$ and $\mathcal{F}_{\text {? }}$. In particular $\left(\mathcal{F}_{1} \cap \mathcal{F}_{2}\right)$-injector $=\mathcal{F}_{1}$-injector $\cap \mathcal{F}_{2}$-injector in each kroup, is nearly always false and we give two simple exampzes.

\subsubsection{Examples}

a) Again let $\mathcal{F}_{p}$ be the class of groups with central p-socle. Put $\mathcal{F}=\mathcal{F}_{2} \cap \mathcal{F}_{3}$. Iet $M_{3} \cong c_{3} \times c_{3}, M_{2} \cong C_{2} \times c_{2}$ and $M=$ $\mathrm{I}_{3} \times \mathrm{M}_{2}$. Ict $\mathrm{A} \cong \Sigma_{3}$ act on $\mathrm{M}_{3}$ as in example 2.3.2 and on $\mathrm{I}_{2}$ so that $M_{2} \Lambda \cong \Sigma_{4}$. Put $G=M A$. Then $G \in \mathcal{F}_{3}$ and $M A_{2}$ is an $\mathcal{F}_{2}$-injector of $G$, but $M$ is the unjque $\mathcal{F}$-injector of $G$.

b) Now put $\mathcal{F}(p)=S_{p} \cdot S_{p} S_{p}$ the clessof Eroups of $p$-length 1 . Let $\mathcal{F}=\mathcal{F}(2) \cap \mathcal{F}(3)$ and let $G$ be a group with unique chief series constructed in the manner of 1.2 .4 from the sequence $3,2,3,2$. 


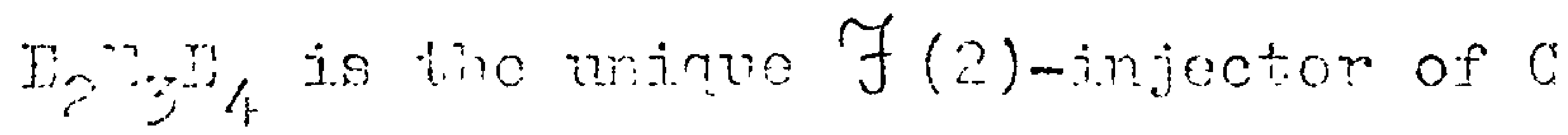

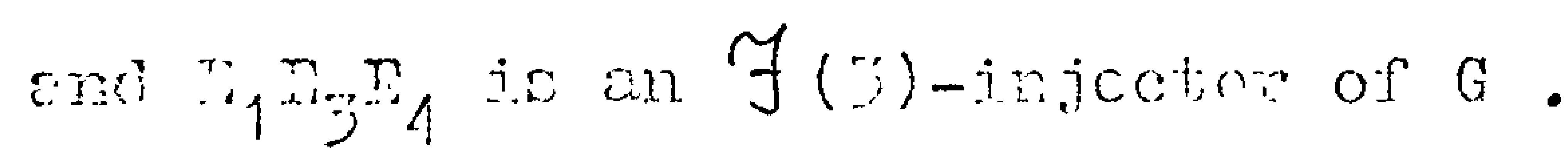

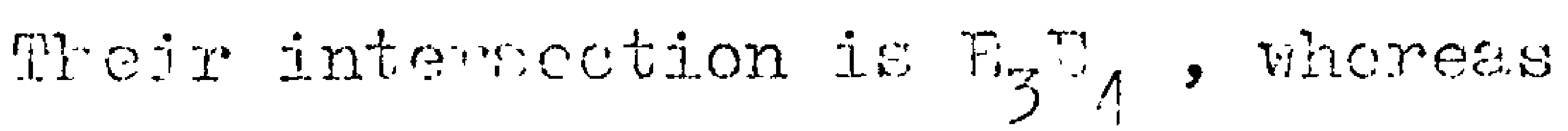

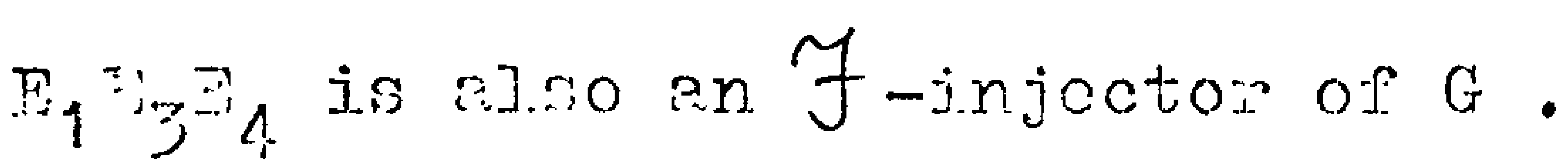

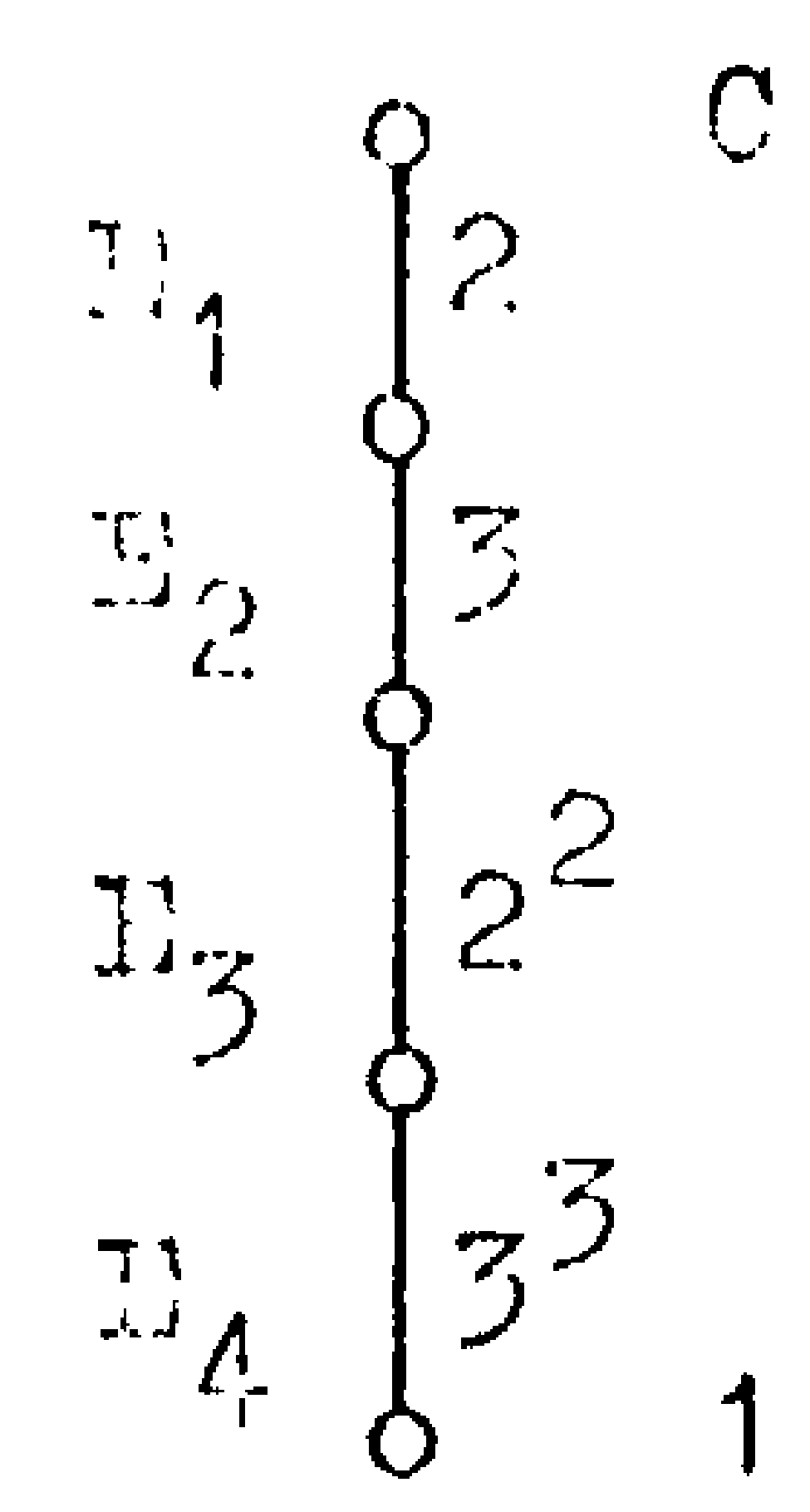

\subsection{Genoretine Pitting clessos}

No discrasion of examples of Fitting clesses could ond rithout sine remerls on this topic. The important generel problem of determining, the smallest Fitting class containing a given sot of groups seems a very thorny ore indeed reflecting an inabjlity to handle normal products. For instance, many moro able minds than our own have been applied to a determination of the pittine cleiss generated by $\Sigma_{3}$, but without succoss. The only real progress in defining a Fj.tting class by a construction process starting with a certain group, was made by Dark in [11]. As we mentioned in 1.2 , the importance of this paper is his construction of a group with a Fischer $\mathcal{F}$-subgroup which is not an 7 -injector. Leaning heavily on Dark's technigues we now construct a family of Fitting classes which, because we are no longer trying to concoct simulteneously a nastily embedded subgroup, are less complicatcd than his class.

\subsubsection{Proposition}

Let $p, q$ and $r$ be primes such that :

a) $p \mid n$ where $n=$ order of $r \bmod q$, and

b) $1<m<n$ and $m / n$ where $m=$ order of $r \bmod p$. Then :

i) The elementary Abelian group $E$ of order $r^{n}$ does not possess commuting automorphisms $\alpha$ and $\beta$ of orders $p$ and $q$ respectively. ii) Ihere exists a group $H$ with the following unique chief series. Furthermore $\mathrm{I}$ is a minimal nomal subgroup of $F$.

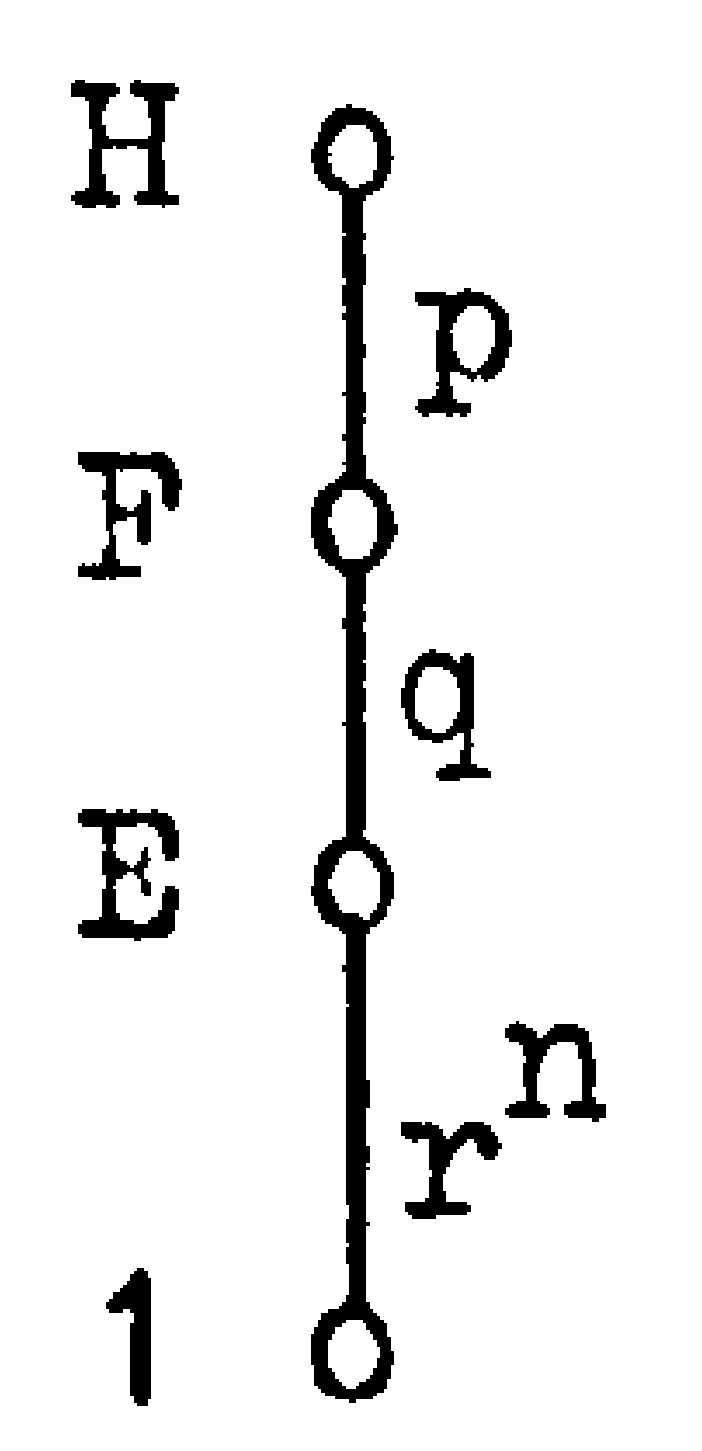


Proor

The followire two atumonts re rel 7-lnown rosults (seo for exsmple soction TI.3 of tuppert's book).

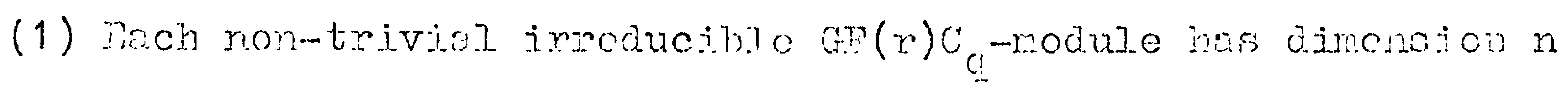

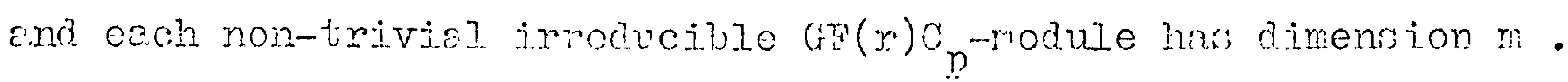

(2) The sct of ald mans from $\left(r^{n}\right)$ to itself of the form $x \rightarrow \lambda x^{r^{t}}$ where $0 \neq \lambda \in G r\left(r^{n}\right)$ and $1 \leqslant t \leqslant N$, is a Eroup of autornorphisms of $\mathrm{GP}\left(\mathrm{r}^{2}\right)^{+}(\cong \mathbb{E})$ which is a split extension of a cyclic group $B$ of order $r^{n}-1$ (the set of nultiplications $\mathrm{x} \rightarrow \lambda \mathrm{x}$ ) by a cyclic Eroup $\mathrm{A}$ of order $\mathrm{n}$ (Eencrated by the Irobcoins avtomorphism $x \rightarrow x^{r}$ ).

i) By assumption b), (1) and Iaschke's theorem, $C_{E}(\alpha)+1$. The fact that $\alpha$ and $\beta$ commute iruplies that $\beta$ normelies $C_{Z}(\alpha)$, but by (1) $\beta$ acts irreducibly on $I$, a contradiction, so such commuting autororphisms do not exist.

ii) Consider the group $A B$ of automorphims of $E$ as in (2). By definition of $n, q \mid x^{n}-1$, so the cyclic group $B$ has a unique subgroup $\langle\mathrm{b}\rangle$ say of order $q$, which by (1) acts irreducibly on $E$. Since $p \mid n$ by supposition, the group $A$ has a subgroup $<a>$ of order $\mathrm{p}$, and $\langle a>$ will normalize $\langle\mathrm{b}\rangle$. By i) this last action is not trivial, and putting $\mathrm{H}=\mathrm{E}\langle\mathrm{b}\rangle\langle\mathrm{a}\rangle$, ii) is established. (Notice that $\mathrm{n} \mid \mathrm{q}-1$ since $\mathrm{r}^{\mathrm{q}-1} \equiv 1 \bmod q$, so certainiy $\mathrm{p} \mid \mathrm{q}-1$. )

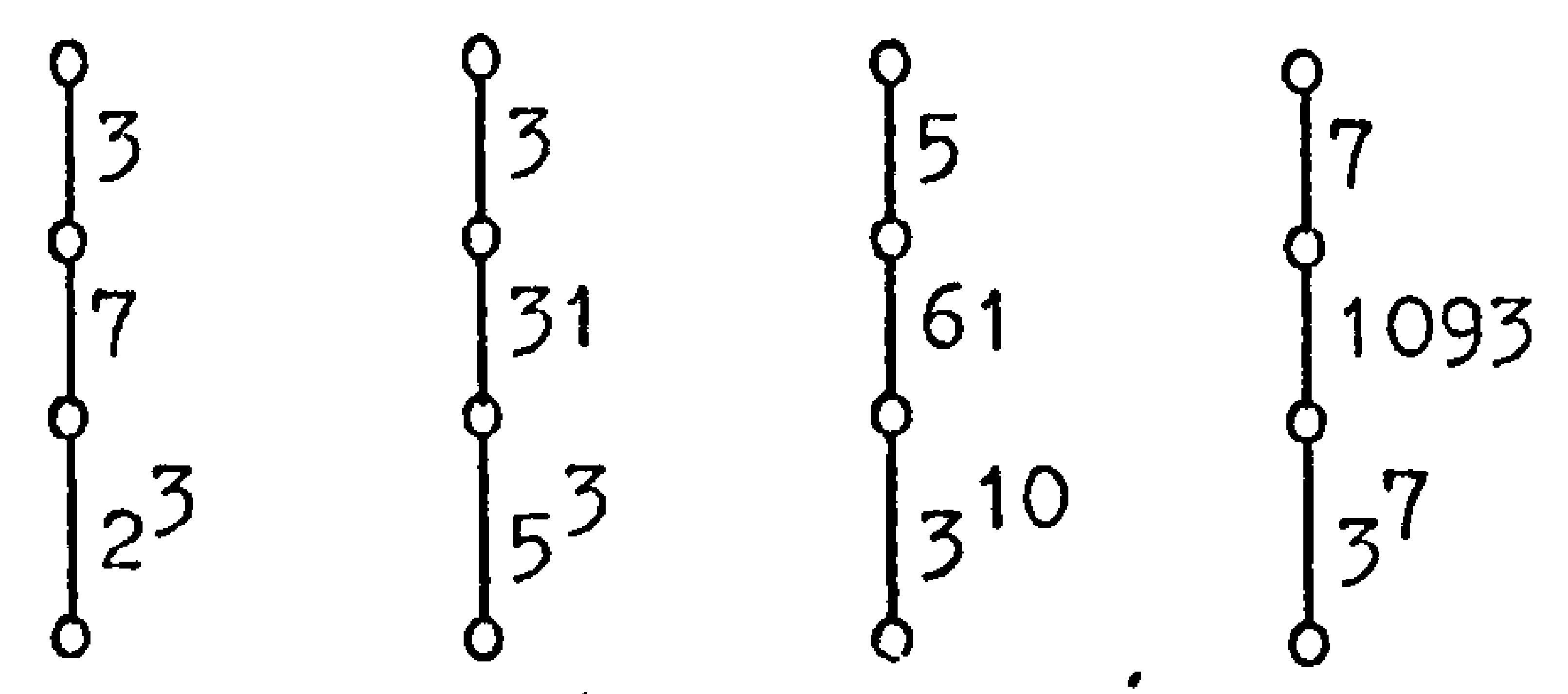

It may be verified thet these are possible forms for $\mathrm{H}$. 


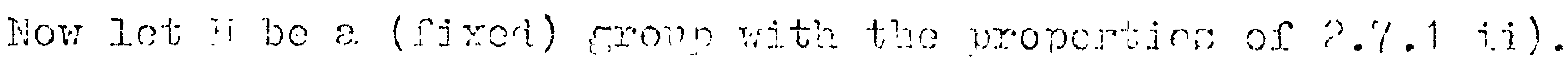

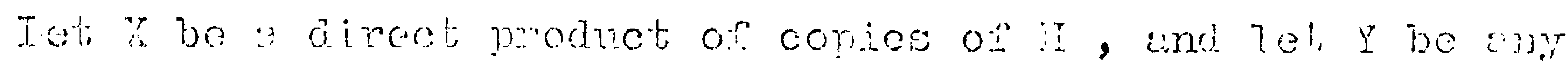
oubrowp of $x$ whose ingex jis a nowor of $p$ and sinch that a sylow p-anberoup $Y_{p}$ of $Y$ acts non-trivielly on the nomel subrioun IT of ccch direct factor It of $X$. Iquivalentily, the inese of $Y_{p}$ onder the notural projection onto sny dixect foctor of $\%$, is non-trivial. Iet $Y$ be the set of all such grouys $Y$ tocether with the identjty gromp. Our aim is to prove that the sot $\left\{G: o^{\prime}(G) / o_{p}(G) \in Y\right\}$ is a Fitting class.

\section{7 .2 Iemma}

If $Y \ni I, M \triangleleft I N=G$ then $G / O_{p}(G) \in Y$.

\section{Proof}

Certainly $1=O_{p}(Y)$ for all $Y \in Y$, so ve may suppose $I$, $I \neq 1$. Let $R$ and $U$ be the $S_{\{r, q\}^{-r a d i c a l s}}$ and $S$ and $V$ the $S_{r}$-radicals of $I$ and $M$ respectively. $R$ i.s a direct product of copies of $F$, $R=F_{1} \times F_{2} \times \ldots \times F_{t}$ say, and since $F$ has trivial centre it follows by an easy argufment that any nomal subgroup of $R$ isomorphic to $F$ must be one of the $F_{i}$. So calling these conies of $F$ occurring as direct factors of $R$ and $U$ ' normal factors : ve nave :

(1) Any automorphism of $R$ (respectively $U$ ) permutes its normal factors.

Let $\Sigma$ bc a fixed Sylow system of $G$,

$\Sigma=\left\{G_{p}, G_{q}, G_{r}, \ldots.\right\}$.

Then $\Sigma$ reduces into $\mathrm{I}, \mathrm{H}, \mathrm{R}, \mathrm{U}, \mathrm{S}, \mathrm{V}$, and

the normal factors of $R$ and $U$. By 1.2.7

$G_{p}=\left(I \cap G_{p}\right)\left(N \cap G_{p}\right)$ etc.,$G_{p}$ normalizes

$G_{q}$ and $G_{q}$ normalizes $G_{r}$. These facts will

be used vithout mention.

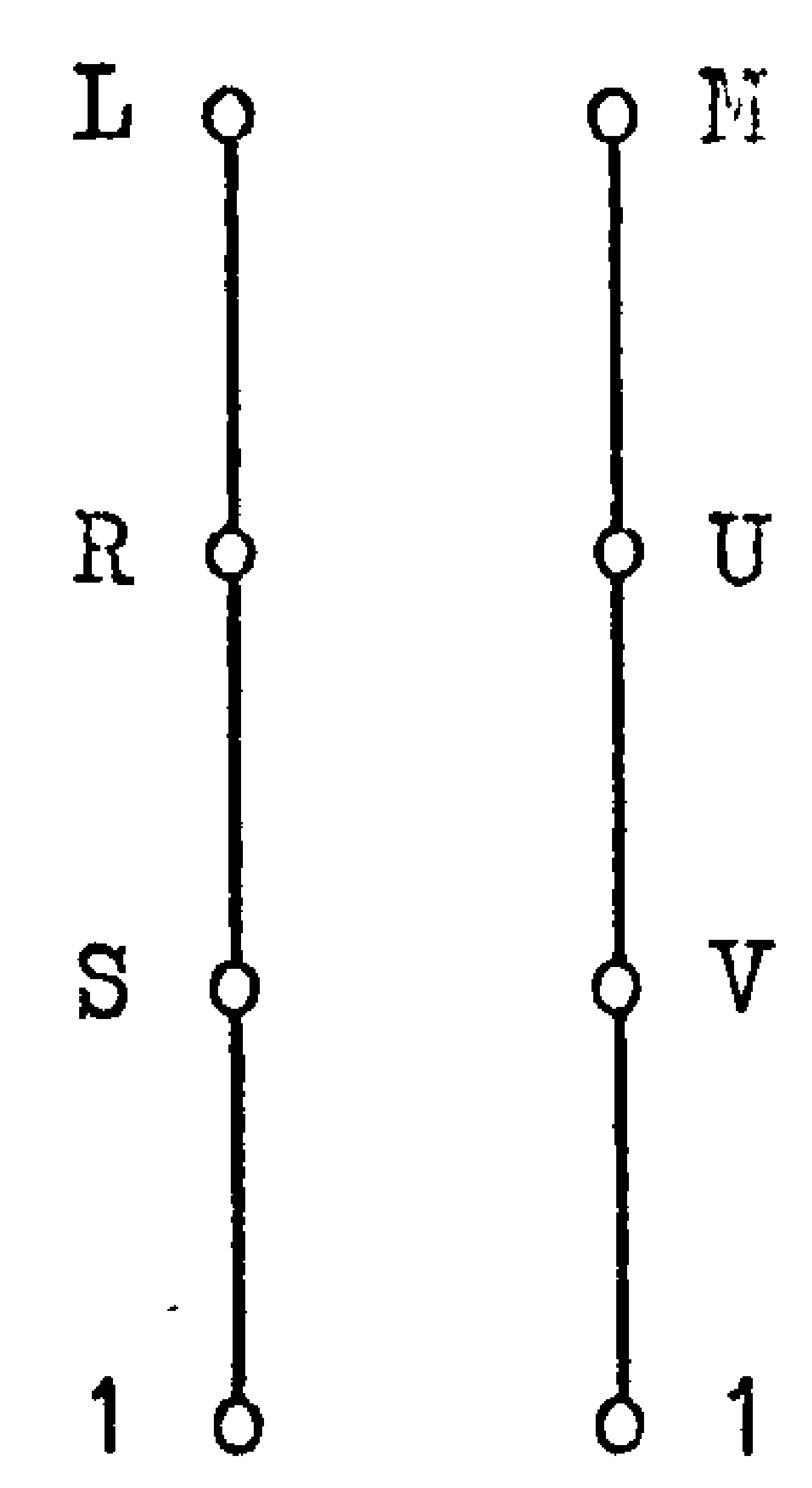

(2) The normal factors of $R$ and $U$ are normal in $R U$. 


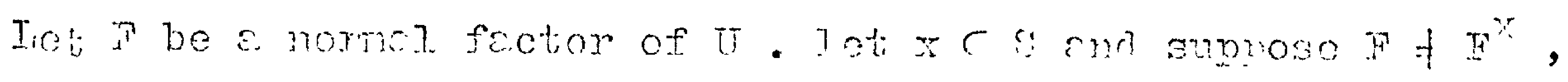

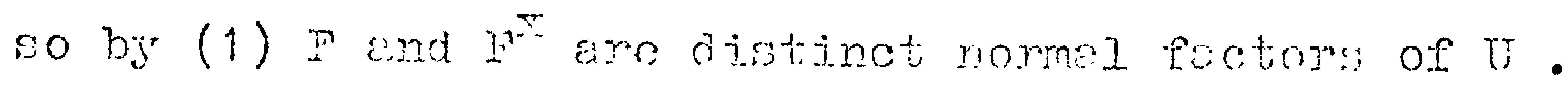

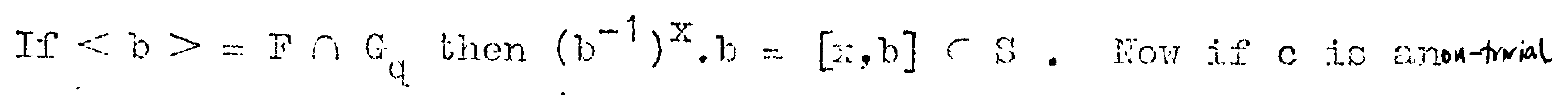
$r$-clerent of of $\mathrm{r},\left(b^{-1}\right)^{x}$ contrejires c aince it lies in $a$ different nomal factor of $U$. Thus $P \exists c^{-1} c^{b}=c^{-1} c^{\left(b^{-1}\right)^{x} \cdot b}=$ $[c,[x, b]] \in S$. But $S$ is Abolion end b centrelizes non-rial $x$-eloment of $F$, theresore $1 \neq[c, b]=[c, b]^{x} \in M \cap F^{x}=1$, a contratiction. Now let, $F_{1}$ be a nomel frctor of $R$ and put $<b_{1}>=F_{1} \cap G_{q}$. Suppose $b_{1}$ does not nomelize $F$, then $F, F b_{1}, \ldots . . . ., F^{b_{1}-1}$ are peirwise distinct normal factors of $U . F_{1} \triangleleft T<I M$, so $\left[b, b_{1}, b_{1}\right] \in U \cap F_{1}$. Since $F \triangleleft U$ this implies that $\left[b, b_{1}, b_{1}\right]$ normalizes $E$. But $\left[b, b_{1}, b_{1}\right]=b_{1}^{-1} b^{-1} b_{1} b_{b_{1}}^{-1} b^{-1} b_{1}{ }^{-1} b_{1} b_{1}$

$$
\begin{aligned}
& =\left(b^{-1}\right)^{b_{1}} b\left(b^{-1}\right)^{b_{1} b_{1}^{2}} \\
& =b \cdot\left(b^{-2}\right)^{b_{1} \cdot b}\left(b_{1}\right)^{2} \text { since } F, F_{1},
\end{aligned}
$$

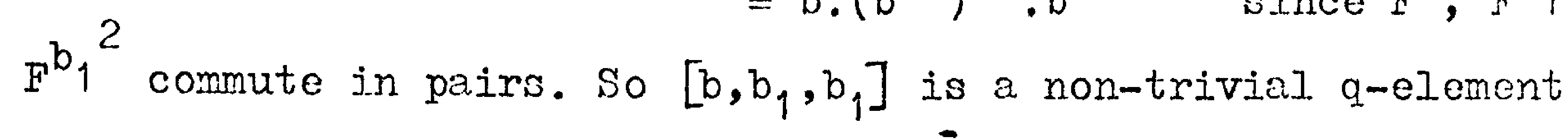
of $F_{1}$ which normalizes $F$. Having shown thot $S$ nomalizes $F$, this implies $F_{1}$ normalizes $F$. Then (2) is an immediate deduction.

(3) If $F_{1}$ and $F$ are normal factors of $R$ and $U$ respectively and $F_{1} \cap F \neq 1$, then $F_{1}=F$.

By (2) $F_{1}$ and $F$ are normai subgroups of $F_{U}$, so $F_{1} \cap F \triangleleft F, F_{1}$. Now $F$ and $F_{1}$ have unique minimal normal subgroups (of order $r^{n}$ ) so $F_{1} \cap F \neq 1$ implies $F_{1} \cap G_{r}=F \cap G_{r}=F$ say.

First suppose $F \leqslant R$, and let $R=F_{1} \times F_{2} \times \ldots \times F_{t}$ be the decomposition of $R$ as the product of its normal factors. Then $<b>=F \cap G_{q} \leqslant R \cap G_{q}=\left(F_{1} \cap G_{q}\right) \times\left(F_{2} \cap G_{q}\right) \times \ldots \times\left(F_{t} \cap G_{q}\right)$ so for $j=2, \ldots, t\left[F_{j} \cap G_{r}, b\right] \leqslant F_{j} \cap G_{r} \cap F=F_{j} \cap E=1$. Therefore $b$ is an element of $R \cap G_{q}$ which centralizes $\left(F_{2} \times \ldots \times F_{t}\right) \cap G_{r}$ which implices $b \in F_{1}$, and hence $F=F_{1}$. 


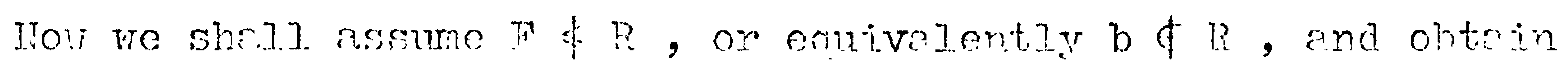
a controcidion. Bry dorintion of the cress $Y$, there existo an element $a_{1} \in T_{i} \cap G_{p}$ wizich acts non-trivituly on $F_{1}$. Then $a_{1}$ norinelizes $\mathrm{E}$, so $\mathrm{N} \leqslant \mathbb{F}^{2} 1$ which is a normal factor of $\mathrm{U}$ by (1). ITor $\mathrm{E}$ lies in a unique normal factor of $\mathrm{U}$, so $a_{1}$ normalizas $i$, whonce it nomalizes $I P \cap G_{q}=\langle b\rangle$, which implies $\left[b, a_{1}\right] \subset F \cap G_{q} \cap I$. But $F \cap G_{q} \cap I=1$ by cur essumption, and therefore $a_{1}$ and $b$ induce commuting autonorphisms of $\mathbb{B}$, acinst 2.7 .1 i), and the proof of (3) is complete.

(4) $\mathrm{RU}=$ (direct product of those nomal factors of $\mathrm{R}$ which intersect $U$ triviajly) $\times$ (direct product of those normel fectors of $U$ which interscct $R$ trivially) $\times$ (direct product of the normal factors of $R \cap U$ ).

First suppose $F_{1}$ is a normal factor of $R$ such that $F_{1} \cap F=1$ for all normal factors $F$ of $U$. Then using (2), $\left[F_{1}, F\right] \leqslant F_{1} \cap F=1$ for all normal factors $F$ of $U$, hence $\left[F_{1}, U\right]=1$ and so $F_{1} \cap U \leqslant Z(U)=1$. Thus for each norma? factor $F_{1}$ of $R$, either $F_{1} \cap U=1$ and $F_{1} U=F_{1} \times U$, or $F_{1} \cap F \neq 1$ for some nomal factor $F$ of $R$ and then $F_{1}=F$ by (3). (4) now follows.

(5) The normal factors of $R$ and $U$ are normal subgroups of IN $=G$. For let $a \in I I \cap G_{p}$, then by (1) a permutes the normal factors of $R$. If $F_{1}$ is a normal factor of $R$ with $F_{1} \neq U$, then $F_{1}^{a} \neq U^{a}=U$ also. Then $\left[F_{1}, a\right] \leqslant F_{1}{ }^{a} F_{1} \cap M=F_{1}{ }^{a} F_{1} \cap U=1$ by (4), so a centralizes $F_{1}$. If $F_{1}$ is a normal factor of $R$ lying in $U$, then it must be a normal factor of $U$ as we saw in (4) , so $F_{1}$, is normalized by a. Thus a nomalizes each normal factor of $R$, and the deduction of (5) is now clear.

Suppose $\mathrm{F}$ is a normal factor of both $\mathrm{R}$ and $\mathrm{U}$. By (5) $\mathrm{F} \triangleleft \mathrm{G}$. 


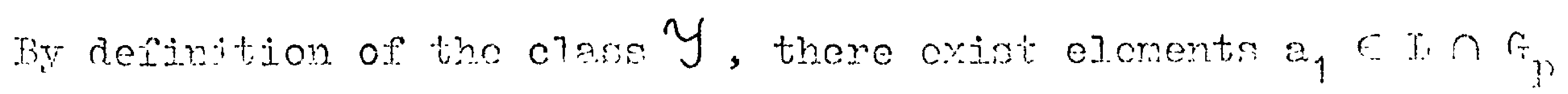

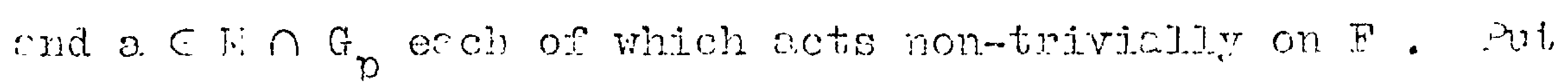
$<b>=F \cap G_{q}$ as wavel. $<a, a_{1}>=P$ say, is a p-rrour which normalizes both $<b>$ and $E-F \cap G_{r}$. If $a^{*} \in P$ centreziros $\langle b\rangle$, thon $a^{*}$ centralizes $\mathrm{Is}$ by 2.7 .1 i). So $\left.\mathrm{C}_{\mathrm{P}}(\mathrm{R})=\mathrm{C}_{\mathrm{p}}<\mathrm{b}\right\rangle$, but $\mathrm{P} / \mathrm{C}_{2}<\mathrm{b}>$ is certainly cyclic and neither a nor $a_{1}$ centralizos $<b>$, so it follovs thot :

(6) $<a$ and $<a_{1}>$ induce the seme outororphisms of $\mathrm{F}$.

Now suppose $\mathrm{F}$ is a nomal factor of $\mathrm{R}$ rith $\mathrm{F} \cap \mathrm{T}=1$. Then by (5) $\left[F, I \cap G_{p}\right] \leqslant R \cap I=I \cap U=1$, so the automorphisms of $F$ induced by $G$ are mercly those induced by $R \cap G_{p}$.

It follows from this and (6) that for eny normel factor $\mathrm{E}$ of $\mathrm{RU}$, the split extension $[F] \mathrm{G}_{\mathrm{p}} / \mathrm{C}_{\mathrm{G}_{\mathrm{p}}}(\mathrm{F})$ is isomorphic to $\mathrm{H}$. Also $\mathrm{C}_{\mathrm{G}_{\mathrm{p}}}(\mathrm{F})$ is a maximal normal subgroup of $G_{p}$ end hence $\mathrm{C}_{\mathrm{G}_{\mathrm{p}}}$ (RU) is a normal subgroup of $G_{p}$ with $G_{p} / C_{G_{p}}(R U)$ an elementary Abelian p-group. Of course $O_{p}(G)$ centralizes $R U$ and hence $O_{p}(G)=C_{G}(R U)$. We have therefore established that $G / O_{p}(G) \in \mathcal{Y}$ as required.

\section{$\underline{2.7 .3 \quad \text { Iemma }}$}

If $W$ sn $\dot{Y} \in Y$ and $W$ is generated by its $r$-elements then $W \in Y$. Proof

Let $W \triangleleft W_{1} \triangleleft W_{2} \triangleleft \ldots \triangleleft W_{n}=Y \in Y$, then by well-knorm results

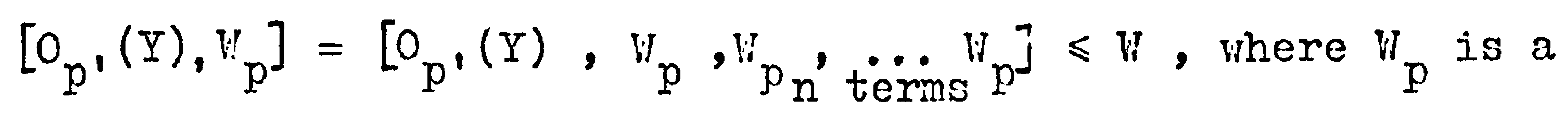
Sylow p-subgroup of $W$. From the structure of $H$ it is clear that the normal subgroup $\left[\mathrm{O}_{p^{\prime}}(\mathrm{H}), \mathrm{H}_{\mathrm{p}}\right]$ must coincide with $\mathrm{O}_{\mathrm{p}^{\prime}}(\mathrm{H})$. It follows that $\left[O_{p},(Y), \pi_{p}\right]=K$ say, is precisely the product of the normal factors of $Y$ on which $V_{p}$ acts non-trivially, in particular $K I_{p} \in Y$. We have $K N_{p} \leqslant W$, on the other hand $K \cdot I_{p} \triangleleft Y$ : 


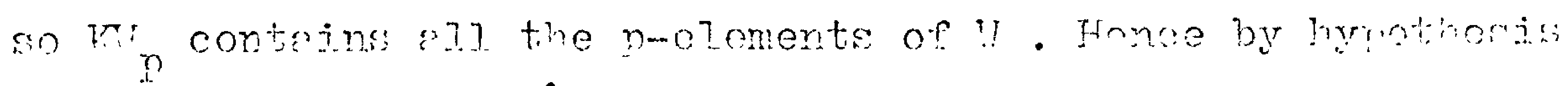

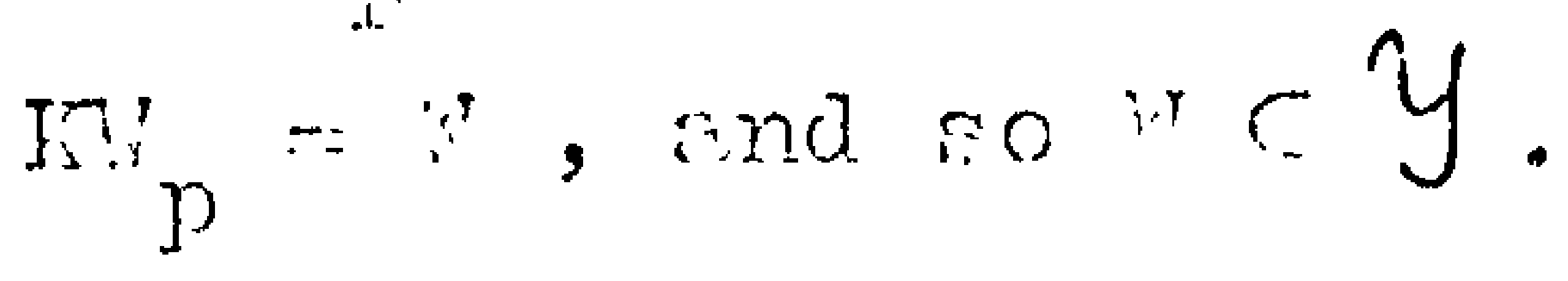

\section{$2.7 .4 \quad 5100025$}

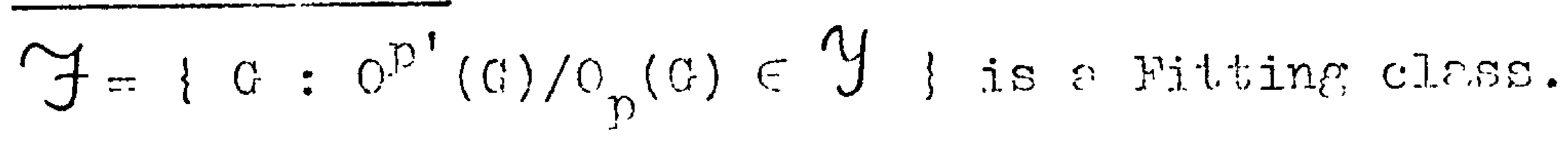

ProOF

Suppose $\mathcal{F} \exists \mathrm{N}_{1}, \mathrm{~N}_{2} \varangle \mathrm{N}_{1} \mathrm{~N}_{2}=\mathrm{G}$. Fhen by 2.5 .1

$$
\begin{aligned}
O^{p^{\prime}}(G) / O_{p}(G) & =O^{p^{\prime}}\left(N_{1}\right) O^{n^{\prime}}\left(I_{2}\right) / O_{p}(G) \\
& =O^{p^{\prime}}\left(\mathbb{N}_{1}\right) O_{p}(G) / O_{p}(G) \cdot O^{\prime \prime}\left(N_{2}\right) O_{p}(G) / O_{p}(G)
\end{aligned}
$$

and $O^{P^{\prime}}\left(I_{i}\right) O_{p}(G) / O_{p}(G) \cong O^{p^{\prime}}\left(I_{i}\right) / O^{P^{\prime}}\left(G_{i}\right) \cap O_{p}(G)$

$=0^{p^{\prime}}\left(N_{i}\right) / O_{p}\left(N_{i}\right) \in Y$, so by 2.7.2 $o^{p^{\prime}}(G) / O_{p}(G) \in Y$.

Suppose $N \triangleleft G \in \mathcal{F}$. Then $0^{n^{\prime}}(\mathbb{N}) / O_{p}(N)=O^{p^{\prime}}(\mathrm{Ii}) / O_{p}(G) \cap$ it

$\triangleleft O^{p^{\prime}}(G) \cap N / O_{p}(G) \cap N \cong O_{p}(G)\left(O^{p^{\prime}}(G) \cap N\right) / O_{p}(G) \triangleleft O^{p^{\prime}}(G) / O_{p}(G)$.

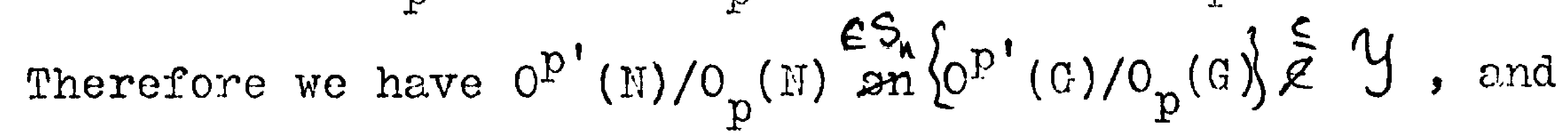
$o^{p^{\prime}}(N) / o_{p}(N)$ is clearly generated by jts p-elcments, so by 2.7 .3 it lies in $Y$.

\subsection{A problem of Gaschütr,}

As an application of 2.7.4 we now settle Test Problem 8.6 of [7] which asks : If $\mathcal{F}$ is a Fitting class, does $Z \leqslant Z(G) \cap G^{\prime}$ and $G / Z \in \mathcal{F}$ imply $G \in \mathcal{F}$. Our answer is no. Since $Z(G) \cap O H \leqslant \phi(G)$ for each $G$, the question is an attempt to discover the rôle of the Frattini subgroup in Fitting class theory.

A $p$-group $P$ is called extra-special when $P / P '$ is elementary Abelian and $\mathrm{P}^{\prime}=Z(P) \cong C_{p}$. It is vell-known (see for example page 204 of Gorenstein's book) that for arbitrary $n$ and $p \neq 2$, there exists a unique (up to isomorphism) extra-special p-croup $p$ of exponent $p$ and order $p^{2 n+1}$. 
p is conemater $7 \because$ elomenlo $\left\{x_{1}, y_{1}, x_{2}, y_{2}, \ldots, x_{n}, r_{n}\right\}$ subject to the relations:

$$
\begin{aligned}
& E^{n}=1 \text { for a.t. } \because \in \in \text {, } \\
& \left.*\left[x_{i}, x_{j}\right]=\left[x_{i}, y_{j}\right]=\left[y_{i}, y_{i j}\right]=1 \text { for } a\right] \\
& i, j \subset\{1, \ldots, n\} \text { vith } i \neq j \text {, }
\end{aligned}
$$$$
\left.1+\left[x_{i}, y_{i}\right]=z \text { sey for } a\right] j i \in\{1, \ldots, n\} \text { and } z \in Z(I) \text {. }
$$

Let - denote tho raturel cpimorphism $P \rightarrow P / \mathrm{P}$. $\overline{\mathrm{P}}$ can bo regarded as a vector space $V$ say, over $G(p)$ with basis $\left\{\bar{x}_{1}, \bar{y}_{1}, \ldots \bar{x}_{n}, \bar{y}_{n}\right\}$ and the map $f: V \times V \rightarrow$ GP $(p)$ refined by $\left[g_{1}, \varepsilon_{2}\right]=z^{f\left(\hat{\varepsilon}_{1}, \varepsilon_{2}\right)}$ is a slrew smetric bilinear form on $V$. Indeed $f$ is the form $\left[\begin{array}{ccccc}0 & 1 & 0 & 0 \\ -1 & 0 & 0 & 0 & \\ 0 & 0 & 0 & 1 & \\ 0 & 0 & -1 & 0 & \\ & & & & \ddots\end{array}\right]$ with respect to the Given basis of $V$. Automorphisms of $P$ which centralize $P^{\prime}=\langle z\rangle$ induce isonetries of $\mathrm{V}$, and therefore we hetve a homomorphisin $h: C_{A}\left(P^{\prime}\right) \rightarrow S_{p}(2 n, p)$, whore $A=A$ ut $P$. By a well-known result $C_{A}\left(P / P^{\prime}\right)$ is a $p-g^{\prime} r o u p, s o$ it will centralize $T^{\prime}$, and so $C_{A}\left(P / P^{\prime}\right)$ is the kernel of the map $h$. Conversely if

$$
\left[\begin{array}{llll}
a_{11} & a_{12} & \ldots & a_{12 n} \\
a_{21} & a_{22} & \ldots & a_{22 n} \\
\vdots & & & \\
a_{2 n 1} & \ldots \ldots & a_{2 n} n
\end{array}\right]
$$

is an element of $G I(i n, p)$ leaving the form $f$ invariant, that is a member of $\mathrm{Sp}(2 \mathrm{n}, \mathrm{p})$, then a study of the relations * reveals that

$$
\begin{gathered}
x_{1} \rightarrow x_{1}^{a_{11} y_{1} a_{12}} \ldots \ldots y_{n}^{a_{12 n}} \\
y_{1} \rightarrow x_{1}^{a_{21}} y_{1}^{a_{22}} \ldots \ldots y_{n}^{a_{2} 2 n}
\end{gathered}
$$




$$
y_{n \rightarrow x_{1}} \ln 1 \ldots \ldots y_{n} \operatorname{sen} 2 n
$$

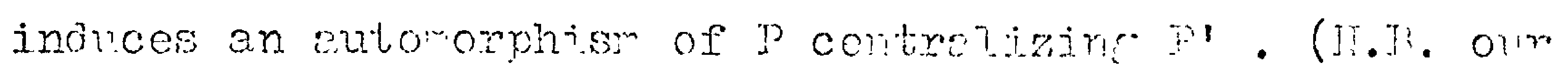
convention of riflt oporabors) menefore vo have a ma $\operatorname{Sp}(2 \mathrm{n}, n) \rightarrow \mathrm{C}_{\Lambda}\left(\mathrm{n}^{\prime}\right)$ which, thourh not necersnxily a horororphism, is cortainly a left invorse for $h$, so it follows that $\operatorname{Sp}(2 n, p) \cong C_{\Lambda}\left(P^{\prime}\right) / C_{f}\left(H^{\prime} / F^{\prime}\right)$. Rernembering that $C_{\Lambda_{L}}(\mu / p)$ is a p-group, an cosy application ci tho schur-ussonhavs theorom (an unccesserily stern measure in the case to follow) then shows thet :

* Nach $p^{\prime}$-subsroup of $3 p(2 n, p)$ is isomornhic to a suidgroup of sut $P$ centrelining $P^{\prime}$.

Ve now apped to the theory of Chevalley groups in order to prove that $\operatorname{Sp}(10,3)$ (the Chevalley group $C_{5}(3)=C$ say) has a subgroup of order 61.5 (sugrested by its order), and we thens: Professor Carter for the following argufment. We are informed that chapters $E$ and $G$ of $[13]$ contain the relevant fects.

The conjugacy classos of meximel tori in a finite Chovalley group correspond 1-1 with the conjugacy classes of elemonts in the Veyl group $W$. In our case, an anisotropic maximal torus $\mathrm{T}_{\mathrm{W}}$ of order $3^{5}+1$ corresponds to a Coxeter element $w$ of order 10 . Now more of the general theory shows that $C_{W}(w)$ is isomorphic to a subgroup of $N_{C}\left(T_{V}\right) / T_{V}$, and so in particular there exists an element of order 5 in $C$ which normalizes $T_{w}$. Now $3^{5}+1=61.4$ so $\mathrm{T}_{W}$ certainly has a characteristic subgroup of order 61 , and the existence of a subgroup of orde: 61.5 follows at once.

By ** this implies that Aut $\left(\mathrm{E}^{*}\right)$ contains a subgroup $\mathrm{J}$ say, of order 61.5 , where $\mathrm{E}^{*}$ is the extra-special group of exponent 3 and order $3^{11} \cdot \mathrm{J}$ centralizes $\mathrm{Z}\left(\mathrm{E}^{*}\right)=\mathrm{Z}$ say. 


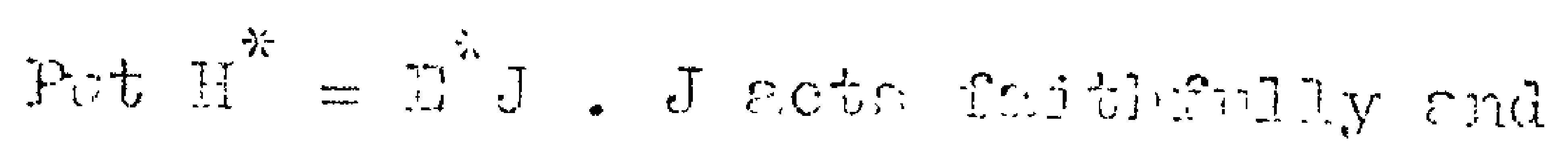

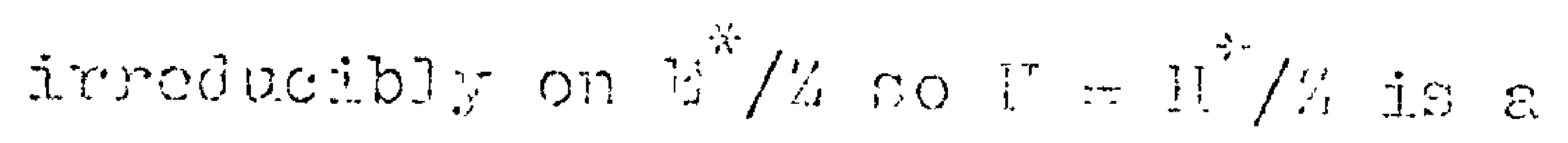

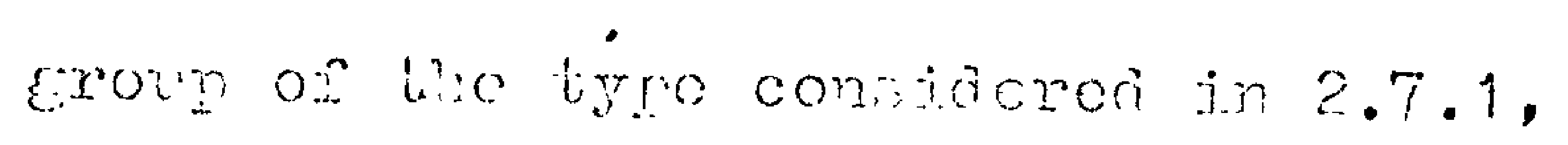
and we mey conetruct a Pittine class $\mathcal{F}$ from this grove es in 2.7. Tt is eesy to check that $\mathrm{H}^{*} \notin \mathcal{F}$, mile

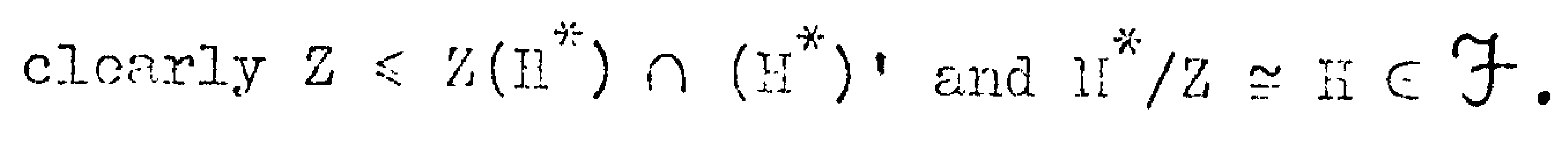
$\left\{\begin{array}{l}I^{* *} \\ 5 \\ 61 \\ \mathrm{I}^{*} \\ 3^{10} \\ z\left(\mathrm{~F}^{*}\right) \\ 3 \\ 1\end{array}\right.$

We remerk that the convcrise statement to Gaschütu's question does not hold either. For, the non-nilpoient eroup $\mathrm{G}=\left[\mathrm{o}_{8}\right] \mathrm{c}_{3}$ Iies in the Fittine class $\mathcal{F}_{2}$ of grouns vith centrat 2-socle whereas $G / Z(G) \cap G^{\prime} \cong \Lambda_{4} \notin \mathcal{F}_{2}$. 
In the first section of this chenten we develop the theorer of pronomality, basing our approach on key theorens of liannAlperin and Fischer. This jmportent topic receives scanty and ununified troatment in the literature. We thon give cur sirnier presentation of nork of Chembers [11], strongthoning his results.

A subgroup $H$ of $G$ is said to be p-normally embedided in $G$ if a sylow p-subgroup of $\mathrm{H}$ is a sylow p-subgroup o." some norme] subgroup of $G$. Ve shall call a subcroup H of G stroncly pronormal in $G$, writton $H$ stropro $G$ if it is p-normally embedded in $G$ for all primes $p$. The importance of this concept in our study of Fitting classes is the previously mentioned fact that the $\mathcal{F}$-injectors of $G$ for a Fischer class $\mathcal{F}$ are strongly pronormal in $G$.

\subsection{Pronormal submroups}

3.1.1 Proposition

a) $H$ pro $G$ and $I I \leqslant I \leqslant G \Rightarrow H$ pro $I$.

b) $\mathrm{H}$ pro $\mathrm{G}$ and $\mathrm{H} \leqslant \mathrm{K} \triangleleft \mathrm{G} \Rightarrow \mathrm{G}=\mathrm{KN} \mathrm{G}(\mathrm{H})$. (Frattini argukment)

c) $\mathrm{H}$ pro, $\mathrm{G}$ and $\mathrm{N} \triangleleft \mathrm{G} \Rightarrow \mathrm{HN}$ pro $\mathrm{G}$, HN $/ \mathrm{N}$ pro $\mathrm{G} / \mathrm{NN}$ and $\mathbb{N}_{G}(\mathrm{HN})=$ $\mathrm{N}_{\mathrm{G}}(\mathrm{HI}) \mathrm{N}$.

d) $H$ pro $G$ and $H \sin G \Leftrightarrow H \triangleleft G$.

e) $H$ pro $G \Rightarrow N_{G}($ ii) is pronormal and self-normalizing in $G$.

f) Hall subsroups and moximal subgroups aro pronormal.

We omit the elementary proofs.

The following result is very useful for verifying pronormality in actual. examples.

\subsubsection{Lemma (Gaschütz)}

Suppose $I J G$ and $H \leqslant G$ such that $H N$ pro $G$ and $H$ pro $N_{G}$ (HiN). 


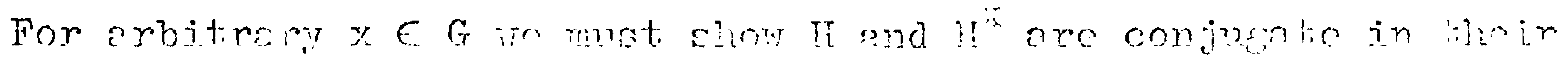

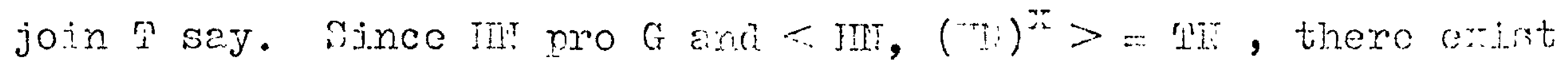

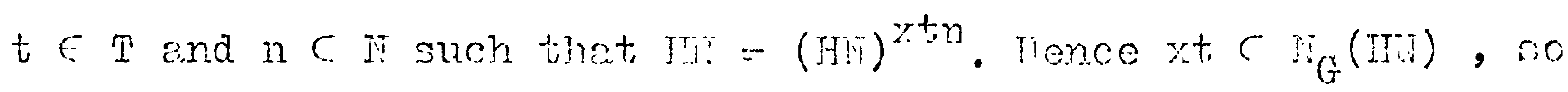
by our hypothesis there crists $\mathrm{y} \in<\mathrm{H}$, II $\mathrm{II}^{\mathrm{xt}}>$ such that $\mathrm{Fi}^{\mathrm{Y}}=$

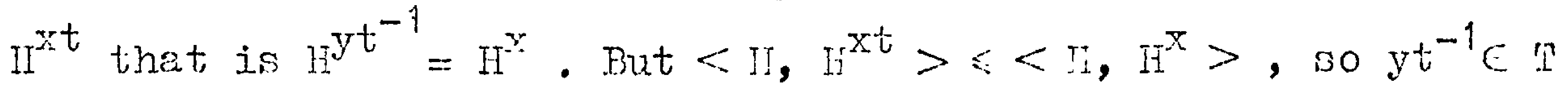
and the proof is complete.

\subsubsection{THEORIT (Niann [15], Alperin)}

$H$ pro $G \Leftrightarrow$ each sylow system $\Sigma$ of 8 reruces into a unique conjugete of $\mathrm{H}$.

PIROOF

We prove the implication $\Rightarrow$ by induction, supposing the result in this direction holds for groups of order less then $|G|$. Suppose the Syrlow systcm $\sum$ of $G$ reduces into $H$ and $\mathrm{H}^{\mathrm{X}}$. Let $M$ be a minimal normal subgroup of $G$. Iset - denote the natural epimorphism $G \rightarrow G / \mathbb{I}$. If $G_{\pi} \in \Sigma$ then by assumption $I \cap G_{\pi}$ is a Hall $\pi$-subgroup of $H$ and so ( $I \cap G_{\pi}$ ) It/ is a IIall $\pi$-subgrong of

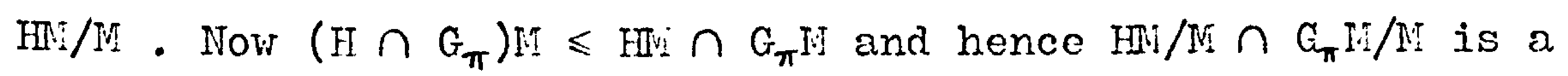
Hall $\pi$-subgroup of $\mathrm{HII} / \mathrm{M}$. Thus $\bar{\Sigma} \backslash \overline{\mathrm{H}}$ and similarly $\bar{\Sigma} \backslash \overline{\mathrm{H}^{\mathrm{X}}}$. 3.1 .1 c) implies $\overline{\mathrm{H}}, \overline{\mathrm{H}^{X}}$ pro $\bar{G}$ so by induction $\overrightarrow{\mathrm{H}}=\overline{\mathrm{H}^{\mathrm{X}}}$ and rence $H M=H^{X} M^{\prime}$. Now $H$ pro $G$ so $H^{X}=H^{m}$ for some $\mathrm{m} \in \mathrm{H}$. By 1.2.7,

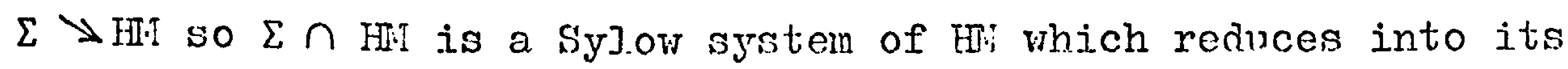
(by 3.1 .1 a)) pronormal suberoups $H$ and $H^{m}$. If In: $<G$ ve may apply our induction hypothesis to deduce $\mathrm{H}=\mathrm{H}^{\mathrm{m}}$. Thus we may assume $\mathrm{H}<\mathrm{G}$ and $\mathrm{HI}^{n}=\mathrm{G}$ for each minimal normal subgroup $\mathrm{II}$ of $\mathrm{G}$. It follows at once that $G$ is a primitive group in which $H$ complements the unique minimal nomal subrroup $M$ of $G$. If $M$ is a p-group then a minimel normel subcroup $\mathrm{N} / \mathrm{M}$ of $\mathrm{G} / \mathrm{I}$ is a $q$-kroup for some $q \neq p$, since $N$ is self-centralizing in $G$. Now if $G_{p}, \in \Sigma$ our supposition implies $G_{p^{\prime}} \leqslant H, H^{\text {In }}$ and so 


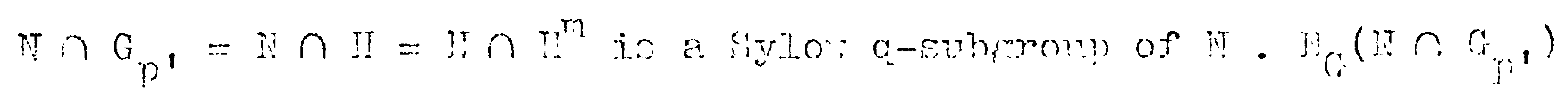

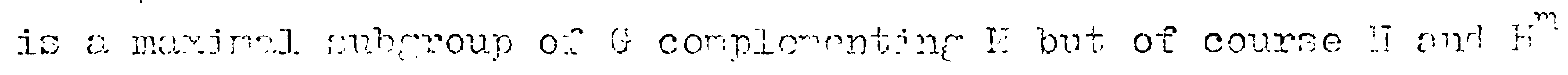

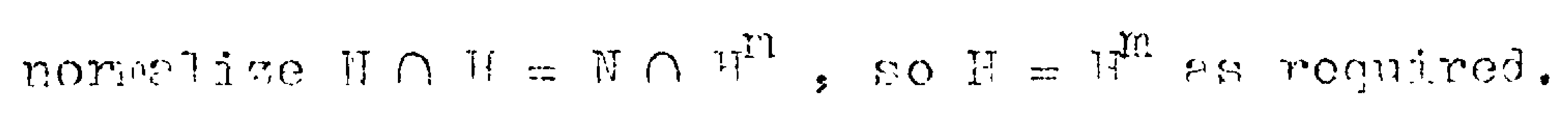

Now conversoly suppose onch sylon system 2 of a roducos into unique conjurete of $H \leqslant G$. Given $x \in G$, pjele $\Sigma$ so that $\Sigma D<H, H^{X}>$, II ( e.s. extend a system of $I$ to e systiem of $<\mathrm{II}, \mathrm{H}^{\mathrm{X}}>$ to a syotem of $\mathrm{C}$ ). Where exists $\mathrm{y} \mathrm{C}<\mathrm{H}, \mathrm{HI}^{\mathrm{X}}>\mathrm{suc} \mathrm{c}$ that $\Sigma \searrow\left(\mathrm{H}^{\mathrm{Y}}\right)^{\mathrm{V}}$, so by suprosition $\mathrm{H}=\mathrm{H}^{\mathrm{Xy}}$ and therefore $\mathrm{E}$ pro $\mathrm{G}$

3.1.4 TISORI! (Fischer, unpublished)

Suppose the Sylow system $\Sigma$ of $G$ reduces into each of tile pronorme.l subgroups $\mathrm{H}_{1}, \mathrm{H}_{2}, \ldots, \mathrm{H}_{\mathrm{n}}$ of $\mathrm{G}$. (So by $3 . \hat{1} .3$ no two of the $\mathrm{I}_{\mathrm{i}}$ are distinct and conjugate.) Put $\mathrm{H}=\left\langle\mathrm{H}_{1}, \mathrm{H}_{2}, \ldots, \mathrm{H}_{\mathrm{n}}\right\rangle$. Then $\mathrm{H}^{\mathrm{G}}$ is the set of minimal (v.r.t. inclusion) elements or $\mathcal{J}=\left\{<\mathrm{H}_{1}^{\mathrm{x}_{1}}, \mathrm{H}_{2}^{\mathrm{x}_{2}}, \ldots, \mathrm{H}_{\mathrm{n}}^{\mathrm{x}_{\mathrm{n}}}>\mathrm{x}_{\mathrm{i}} \in \mathrm{G}\right\}$ and $\Sigma \mathrm{y}_{\mathrm{H}}$ pro $\mathrm{C}$. PROOF

Let $\mathrm{T}=\left\langle\mathrm{H}_{1}{ }^{\mathrm{x}_{1}}, \mathrm{H}_{2}^{\mathrm{x}_{2}}, \ldots \mathrm{H}_{\mathrm{n}}{ }^{\mathrm{x}_{n}}>\right.$ be an arbitrary nember of $\mathrm{Y}$. Suppose $\Sigma^{g} \searrow I$. Certainly $\Sigma^{g} \searrow \mathrm{H}_{i}$ for all $i$. $\Sigma^{g} \cap \mathrm{T}$ reauces into some conjugate of $\mathrm{H}_{i}{ }^{\mathrm{E}}$ in $\mathrm{T}$, say $\mathrm{H}_{i}{ }_{i} t_{i}$ where $t_{i} \in T$. But then $\Sigma^{E} \rightarrow H_{i}{ }^{x_{i}} t_{i}$, so by $3.1 .3 \mathrm{H}_{i}{ }^{g}=H_{i}{ }^{x_{i}}{ }^{t_{i}}$. Therefore $\mathrm{H}^{g}=\left\langle\mathrm{H}_{1}{ }^{g}, \mathrm{H}_{2}^{g}, \ldots, \mathrm{H}_{\mathrm{n}}^{g}\right\rangle \leqslant \mathrm{T}$ anc we have proved the first part of the statement.

Putting $x_{1}=x_{2}=\ldots=x_{n}=1$, we have $H=T$ and so $H^{E} \leqslant T$ implies $\mathrm{H}=\mathrm{H}^{\mathrm{g}}$. Therefore $\Sigma^{\mathrm{E}} \searrow \mathrm{H}=\mathrm{H}^{\mathrm{g}}$ and hence $\Sigma \searrow \mathrm{H}$. If also $\Sigma \searrow H^{X}$, then the above argupment yields $H_{i} \leqslant H^{X}$ for all $i$ and so $H \leqslant H^{X}$. Therefore $H=H^{X}$ and by $3.1 .3 H$ pro $G$.

As an immediate corollary of 1.2 .7 we have :

3.1 .5 Proposition

If the sylow system $\Sigma$ of $G$ reduces into subgroups $A$ and $B$, and $A \perp B$, thein $\Sigma \searrow A B, A \cap B$. 


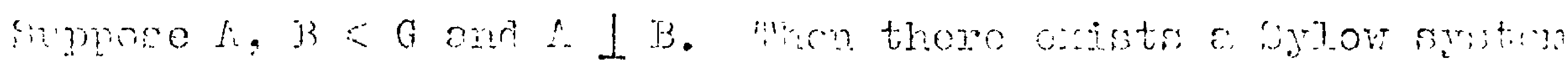

$\Gamma$ of a wion reduces inte is mo $D$ (and honce alro inton is a ra

$A \cap B$ by 3.1.j). Forcover in $A$, Bro $C$, then $A 3$ gro $G$. Proof

Let $\Sigma_{0}$ be a sylow sivitom of is which roducos into s. Then for some ab $\in A B, \Sigma_{0} a b>B$ so $\Sigma_{0}^{a}>B^{b^{-1}}=B$. Lut $\Sigma_{0}^{a} \gg A^{a}=L_{\text {, }}$ so extending $\Sigma_{0}{ }^{a}$ to a systion $s$ of $G$ re have $\Sigma>A, B$. If $A, B$ pro $G$, then by $3.1 .4, \Lambda B=\langle A, B>$ pro $G$.

3.1 .7 Remeriss

a) 3.1 .3 and 3.1 .6 show thet no two distinct conjugates of a pronormal subsroup permute.

b) Thore crists a non-solviblo group $G$ rith a pair of remutible pronormal subgroups $A$ and $B$ such that $A B$ is not pronormal in $G$. An example in a paper of Philip Hall cited by Chambers in [14] exhibits this. The simple group $I=\operatorname{PSI}(2,7)$ of order $168=$ 7.8.3 has two distinct conjugecy classes of subsroups of order 24 which are interchanged by an involutory automorphism $x$ n $I$. Let $\mathrm{H}$ and $\mathrm{H}^{\mathrm{X}}$ be representatives of these classes of subgroups of I. Put $G=\langle I, x\rangle$. H j.s a product of Sylow 2- and 3-suberroups of I and those are of course pronormal in G. However, as has bcen stated $H$ and $H^{X}$ are not conjugate in $I$, so neither are they conjugate in its subgroup $\left\langle\mathrm{H}, \mathrm{H}^{\mathrm{X}}\right\rangle$.

c) An important consequence of 3.1 .3 is that a fixed sylow system of $G$ determines a unique member of each conjugacy class of pronormal subgroups of $G$, and in many investigations it suifices to focus attention on these ropresentatives. For instance, suppose $\Lambda$ and $B$ are (distinct) pronormal subgroups of $G$ determined in this way, then if conjugates $\Lambda^{\mathrm{X}}$ and $\mathrm{B}^{\mathrm{Y}} \mathrm{say}$; 


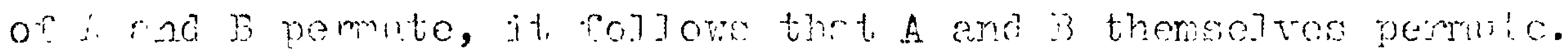

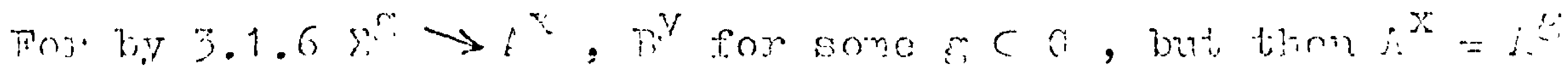

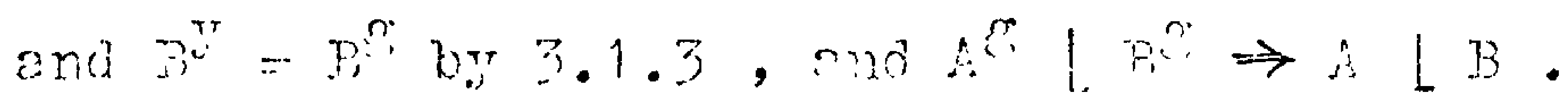

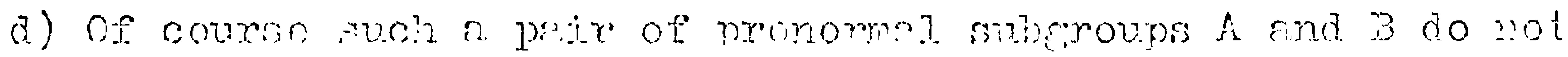
necosemily pernute. For exemple, rith tho aid of 3.1 .2 it is onity to show thet $<(1234)>$ and $<(123)>$ are pronomel sunnoura

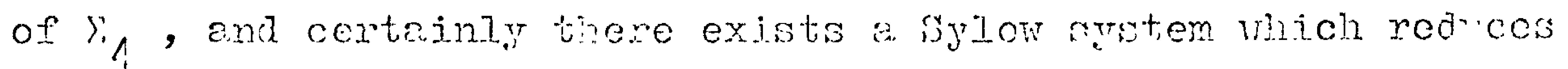
into them both but thar do not permute.

e) If $A ; B$ pro $G$ and $\Lambda \perp B$, thon it does not follon that $A \cap B$ is nronomnat in $G$. For as in $d)<(1234)>$ pro $_{4}$ and $<(12)(34),(13)(24)><\Sigma_{4}$, althom their intersection $<(13)(24)>$ is subnormal but not normal, and honce by 3.1 .1 d) cennot be pronormal.

\subsubsection{Proposition}

As in 3.1 .4 , suppose $\Sigma \longrightarrow \mathrm{H}_{1}, \mathrm{H}_{2}, \ldots, \mathrm{HI}_{\mathrm{n}}$ pro $\mathrm{G}$ and $\mathrm{H}=$ $<H_{1}, H_{2}, \ldots, H_{n}>$. Assume further that for some $x_{i} \in G$ $\mathrm{H}_{1}^{\mathrm{x}_{1}}, \mathrm{H}_{2}^{\mathrm{x}_{2}}, \ldots, \mathrm{H}_{\mathrm{n}}^{\mathrm{x}_{n}}$ permute in pairs and let $\mathrm{K}$ be their product. Then $\mathrm{K}$ and II are conjugate and in particular $\mathrm{K}$ pro $G$ by 3.1 .4 .

\section{Proof}

By remark 3.1 .7 c) $\mathrm{H}_{1}, \mathrm{H}_{2}, \ldots, \mathrm{H}_{n}$ permute in pairs, so $\mathrm{H}=$ $\mathrm{H}_{1} \mathrm{H}_{2} \ldots \mathrm{H}_{n}$. If $\mathrm{n}=2$, then by 3.1 .6 there exists $\xi \in$ G such that $\Sigma^{g}>\mathrm{H}_{1}{ }^{\mathrm{x}_{1}}, \mathrm{H}_{2}^{\mathrm{x}_{2}}$, so as before $\mathrm{H}_{1}^{\mathrm{X}_{1}}=\mathrm{H}_{1}$, and $\mathrm{H}_{2}{ }^{\mathrm{X}_{2}}-\mathrm{H}_{2} \mathrm{~g}$ by 3.1.3. Honce $\mathrm{H}^{\delta}=\mathrm{K}$. Now suppose $\mathrm{n}>2$ and procced by indiuction

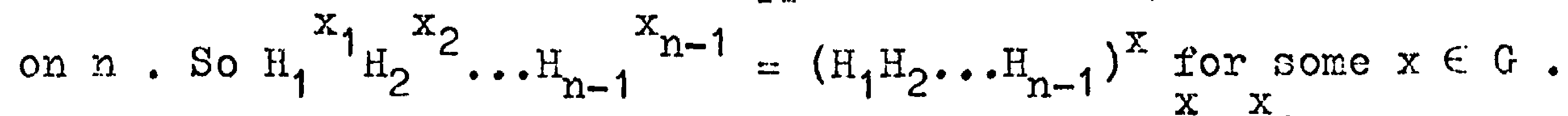

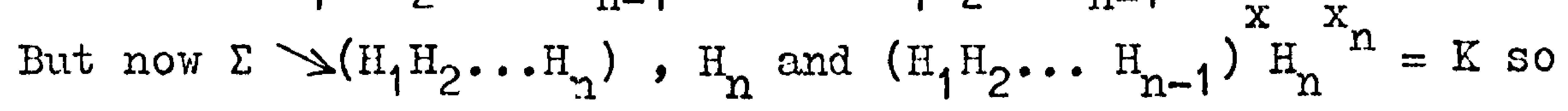
again by induction $\mathrm{K}$ is conjugate to $\mathrm{H}$ as required.

\subsubsection{Rernarks}

a) It can happen that a group $G$ has no Sylow system which reduces into every member of a given set of pairvise permutable pronormal subgroups of $G$. 


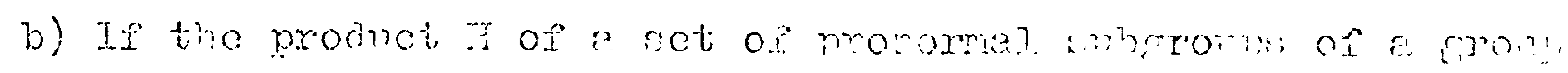

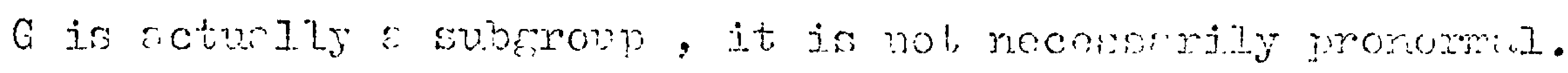

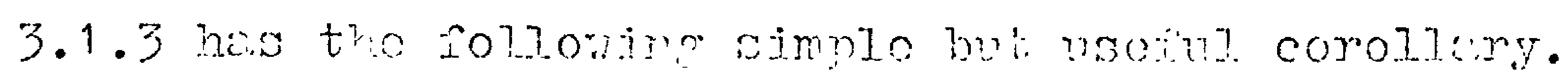

3.1 .10 Troposition

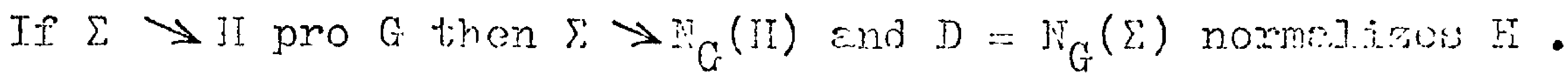
Proof

Certeinly $\Sigma \gg\left(\mathrm{HT}_{G}(\mathrm{H})\right)^{\mathbb{E}}$ for some $\beta \in G$.

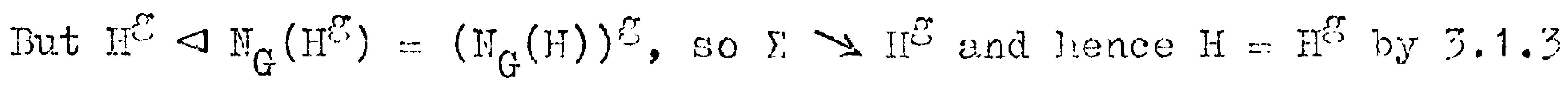
giving $\Sigma \searrow N_{G}(H)$.

If $d \in D$ then $\Sigma=\Sigma^{d} \longrightarrow \mathrm{H}^{\mathrm{d}}$, so.agein by $3.1 .3 \mathrm{H}=\mathrm{T}^{\mathrm{d}}$.

\section{2 strongly pronomal subsroups}

Suppose $H$ is a p-norme.lly embedded subgroun of $G$. So a sylow $\mathrm{p}-\mathrm{sub}$ group $\mathrm{H}_{\mathrm{p}}$ of $\mathrm{H}$ is a sylow p-subgroup of some normal subrroup IN say, of $G$ ( $N$ could clearly be telien to be $<\mathrm{H}_{\mathrm{p}}{ }^{\mathrm{G}}>$ ) and is therefore pronormal in $G$. If $X / Y$ is a $p$-chief factor of $G$ then $\mathbb{N}$ (being normol in $G$ ) either covers or avoids it. In case $N$ covers $X / Y$ then so does its Sylow $p-s u b g r o u p ~ H_{p}$, and henco $H$ covers $X / Y$. If $N$ avoids $X / Y$, so too does $\mathrm{I}_{p}$ and therefore II must also. We have shown that $H$ covers or avojds each p-chiof factor of $G$.

Now let $H$ stropro $G$, so every Sylow subgroup of II is pronormal. in $G$. Suppose further that $K \leqslant G$ such that, for each $p, a$ Sylow p-subgroup of $\mathrm{K}$ is conjugate to a sylow p-subgroup of II, in $G$. (We shall call such $K$ locally conjugate to $H$ in $G$. ) By their orders $H$ and $K$ are minimal elements of $\left\{<\mathrm{H}_{p_{1}}^{x_{1}}, \ldots H_{p_{n}}{ }^{x_{n}}>\right.$ : $\left.x_{i} \in G\right\}$ where $\left\{p_{1}, \ldots, p_{n}\right\}$ are the primes dividing $|H|$. Thus by $3.1 .4 \mathrm{H}$ and $\mathrm{K}$ are conjugate pronormal subgroups of $\mathrm{G}$. Again suppose $H$ stropro $G$ and this time that $K$ is a subgroup of $G$ which covers each chief factor of $G$ which $H$ covers and avoids 


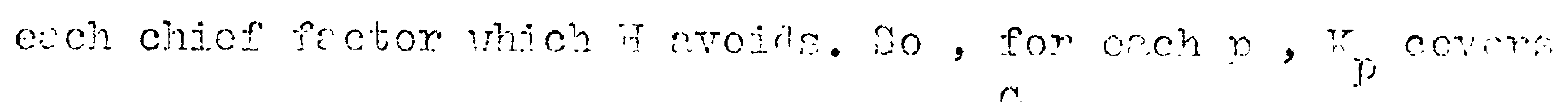

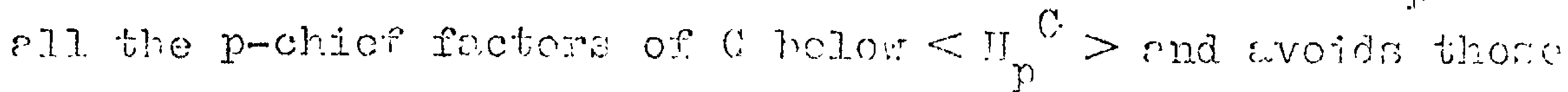

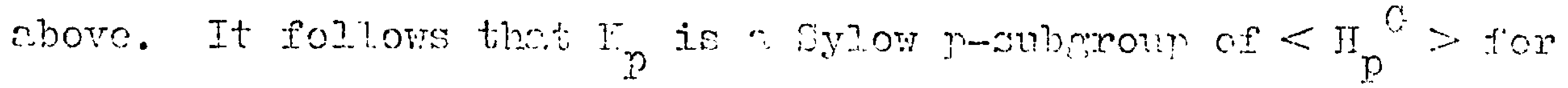

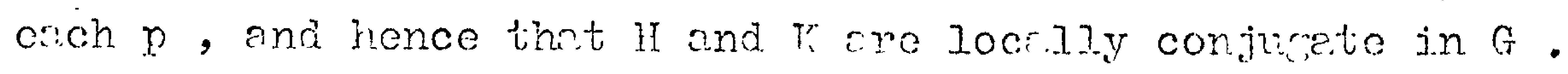
Prom what we have scon enove, II snd $x$ are actually conjugete in 6 .

collecting those rosults end edding sore easily proved focts we heve :

3.2.1 Proposition (Chambers [14])

If $H$ stropro $G$ and $K \leqslant G$ then

a) H covers or avoids each chicf factor of $G$

b) $\mathrm{H}$ pro G

c) $\mathrm{K}$ Iocally conjugate to $\mathrm{H}$ in $\mathrm{G} \Rightarrow \mathrm{K}$ conjugate to $\mathrm{H}$ in $\mathrm{G}$

d) $K$ covers or avoids chief factors in the same mannor as II $\Rightarrow \mathrm{K}$ and $\mathrm{H}$ are conjugate in $\mathrm{G}$

e) $\mathrm{H} \leqslant \mathrm{T} \leqslant \mathrm{G} \Rightarrow \mathrm{H}$ stropro $f \mathrm{~T}$

f) $N \triangleleft G \Rightarrow H N$ stropro $G$ and $H T / N$ stropro $G / N$

g) $N \triangleleft G, N \leqslant T \leqslant G$ and $T / N$ stropro $G / N \Rightarrow T$ stropro $G$.

\subsubsection{Peme.rks}

a) The example in 3.1 .7 b) shows that $\mathrm{H}$ stropro $\mathrm{G}$ does not imply $H$ pro $G$ for non-soluble groups, so our terminology is misleading outside $S$ and is best confined to this class. b) As we remarked in $3.1 . .1 \mathrm{~d}),<(1234)>$ oro $\Sigma_{4}$ but this 2-subgroup is not strongly pronormal in $\Sigma_{4}$.

c) The strongly pronomal p-subgroups of a group $G$ are exactly the Sylow p-subgroups of the normal subgroups of $G$.

\section{2 .3 Lemma}

Let $P_{1}, P_{2}$ stropro $G$ such that $P_{1}, P_{2} \leqslant G_{p}$ a Sylow $p$-subgroup of $G$. Then $P_{1} \perp P_{2}$ and $P_{1} P_{2}, P_{1} \cap P_{2}$ stropro $G$. Proof

Let $N_{i}=\left\langle P_{i}^{G}\right\rangle$ so $P_{i}=G_{p} \cap N_{i}$ for $i=1,2$. Put $N=N_{1} N_{2}$. 
Inon $P_{1} \cap P_{2}=C_{p} \cap\left(M_{1} \cap i_{2}\right)$ rio ${ }_{1} \cap P_{2}$ stromo

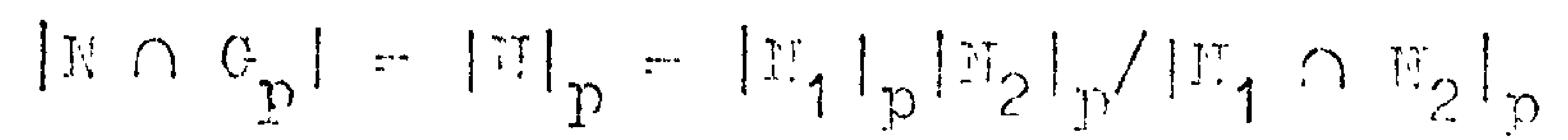

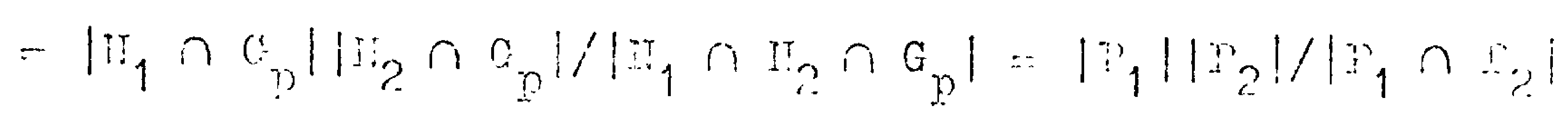

$\therefore\left|P_{1} P_{2}\right|$

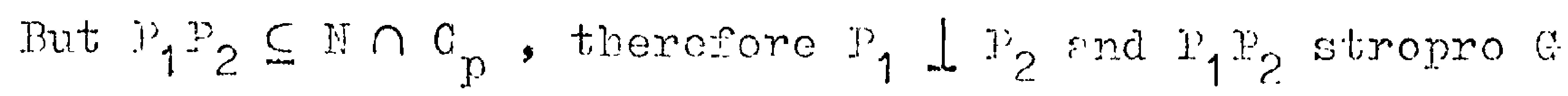

\section{2 .1 Iscinrina}

Iet $\Sigma$ be a Sylor system of $G$. Suppose P, Q stropro $G$ rith $P \leqslant G_{p} \in \Sigma$ and $Q \leqslant G_{q} \in \Sigma$, and $p \neq 0$. Then $P \perp 0$ and

PQ strorro G.

\section{Proof}

The hypotheses imply $P$ is a Syjor p-subgroup of $<P^{G}>{ }^{A} p^{\prime}$, which has $p$-powcr index in $G$, and $Q$ is a sylow $q$-subgroup of $\left\langle Q^{G}>G_{q^{\prime}}\right.$, which has $q$-porer index in $G$, where $G_{p^{\prime}}, G_{q^{\prime}} \in \Sigma$. Hence $G_{\{p, q\}} \cap<e^{G}>G_{p}, \cap<Q^{G}>G_{q^{\prime}}$ is a subgroup of $G$ of order $|P||Q|$ which contains the subset, $P Q$ of $G$, where $G\{p, q\} \in \Sigma$. Therefore $P \perp Q$ and $P Q$ stropro $G$ follows at once.

\section{2 .5 THEORETI}

Suppose the Sylow system $\Sigma$ of $G$ reduces into the strongly pronormaI subgroups $A$ and $B$ of $G$. Then $\Lambda \perp B$ and $A B, A \cap B$ stropro $G$.

\section{PROOF}

Suppose $p_{1}, p_{2}, \ldots, p_{n}$ are the primes dividing $|G|$. For $i=1, \ldots, n$ let $A_{p_{i}}=A \cap G_{p_{i}}, B_{p_{i}}=B \cap G_{p_{i}}$ where $G_{p_{i}} \in \Sigma$. By 3.2 .3 and 3.2 .4 the strongly pronormil subgroups $A_{p_{1}}, \ldots, A_{n}$, ${ }^{B} p_{1}, \ldots B_{p_{n}}$ of $G$ are pairwise permutable and (by order) ${ }^{A} p_{i}{ }^{B} p_{i}$ is a sylow $p_{i}$-subgroup of their product $A B$ and $A_{p_{i}} B_{p_{i}}$ stropro $G$. So $A B$ stropro $G$. By $3.1 .5 G_{p} \cap(A \cap B)=A_{p} \cap B_{p}$ is a sy] ow p-subgroup of $A \cap B$, and by $3.2 .3 A_{p} \cap B_{p}$ stropro G.. Thus $A \cap B$ stropro $G$. 


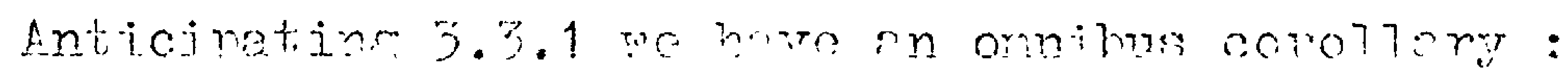

3.?.6 Coro.17exy

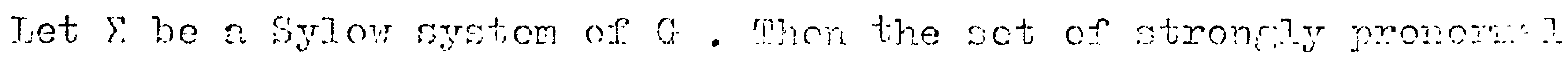
subrowns of $G$ into which $\Sigma$ reducos is a Jettice where join in product, which includes 211 normel. singrouns of G and an injoctor for onch fischer closs.

\subsection{Bittins clrosos wjth strongly nronomol injectors}

3.3 .1 TImORt: (Tischer)

If $\mathcal{F}$ is a Fischor class and $V$ is an $\mathcal{F}$-injector of $G$ then $V$ stropro $G$.

\section{PROOF}

We choose and fix a prime $p$, and show that the hypothescs imply $V$ is p-normally embedded in $G$. Tet $G$ be a group of miniral order for which this is false. Put $K=G_{7 p}$. By definition of $\mathcal{F}^{p}$, the $\mathcal{F}$-injector $V \cap K$ of $K$ contains a. Sylow p-subsoup of $K$ and hence $K<G$. Let $I=0_{p}(G \bmod K)$. Again by definition of $\mathcal{F}^{p}$, we have $o_{p^{\prime}}(G / K)=1$, so $I / K=E(G / K) \neq 1$.

First suppose $I=G$, so $G / K$ is a $p$-group and hence $K V$ sn $G$. But of course $V$ pro $G$, so KV pro $G$ by $3.1 .1 \mathrm{c})$. Then $3.1 .1 \mathrm{~d}$ ) shows $K V \triangleleft G$ and it follows that $V$ contains a, sylow p-subgroup of KV against the assumption that $G$ is a counterexample. Therefore $I \neq G$ and we let $N$ be a maximal nomal subgroup of $G$ containing $I_{1}$.

If $V_{p}$ is a sylow p-subgroup of $V$, then $V_{p} \cap N$ is a sylow p-subiroup of $V \cap N$ which is an $\mathcal{F}$-injector of $N$. By the minimality of $G, V_{p} \cap \mathbb{N}$ is a sylow p-subgroup of $\left\langle\left(V_{p} \cap N\right)^{N}\right\rangle$. Now it is clear that the Sylow p-subgroups of the $I\left\{\begin{array}{l}O_{p}(G \bmod K) \\ p \\ x \\ 1\end{array}\right\}^{G p}$ F-injectors of an arbitrary group form 


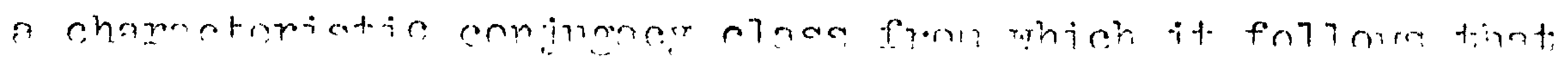

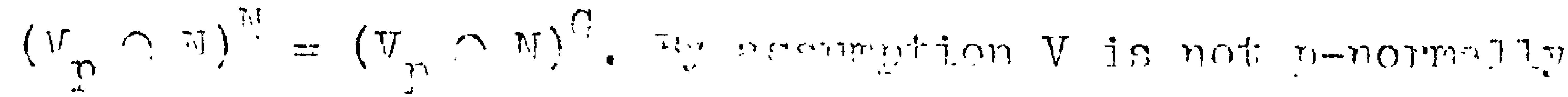

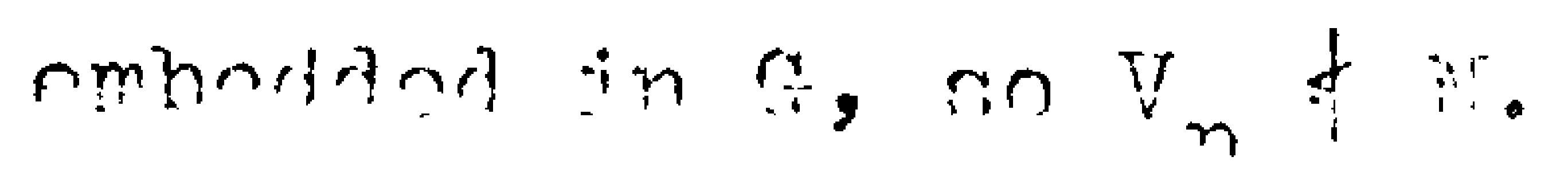

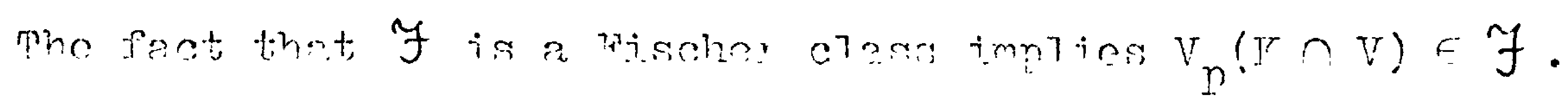

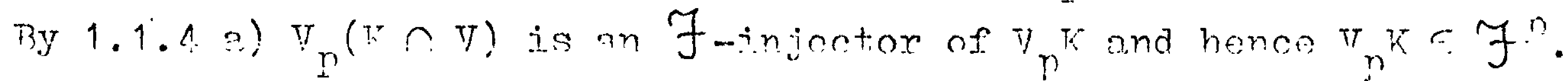

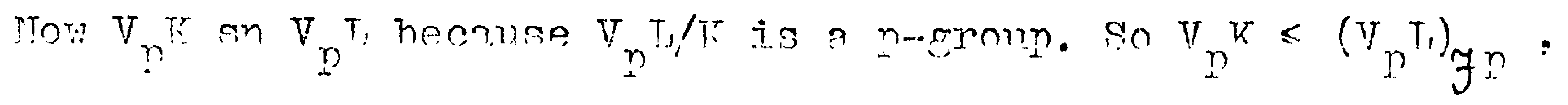

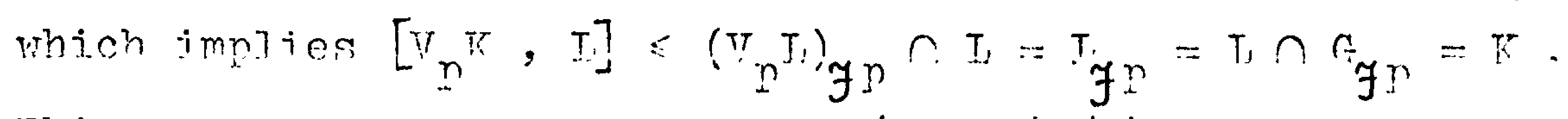

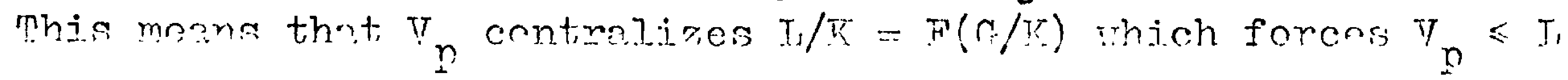

as usual. Thjs finel contimadction shore no such g orists.

so 7 -injectors are p-nomml1.y cmberred for ell primes n and

the theorem is estoblished.

As en immediste consecuence of 3.2 .1 d) re have :

\subsubsection{Corol7ary (Chamhers $[11]$ )}

The $\mathcal{F}$-injectors of a sroup $G$ for a Fischer class $\mathcal{F}$ are characterjoed by their corcring and avoidance of the chinf factors of $G$.

Unfortunately this does not hold for all. Fitting classes.

\section{3 .3 Wxample}

Let $I \cong \mathrm{C}_{3} \times \mathrm{C}_{3}$ be the natural module (under right action) for $G T(2,3) \doteq H$ say, and put $G=M H$.

$H \cong\left[0,8 \Gamma_{3}\right.$ and $G$ has the unique chief series shorm. Let $A=<\left[\begin{array}{ll}1 & 1 \\ 0 & 1\end{array}\right],\left[\begin{array}{l}20 \\ 01\end{array}\right]>$

and $B=<\left[\begin{array}{ll}1 & 1 \\ 01\end{array}\right]\left[\begin{array}{l}10 \\ 02\end{array}\right]>$, subgroups of $\mathrm{H}$.

$\Gamma_{3} \simeq \mathrm{A}, \mathrm{B}$ and both complement $\mathrm{N}$ in $\mathrm{G}$.

$C_{M}(A)=\langle(0,1)\rangle$ is the unique minimal normal

subgroun of $M A$ whereas $C_{M}(B)$ is triviel.

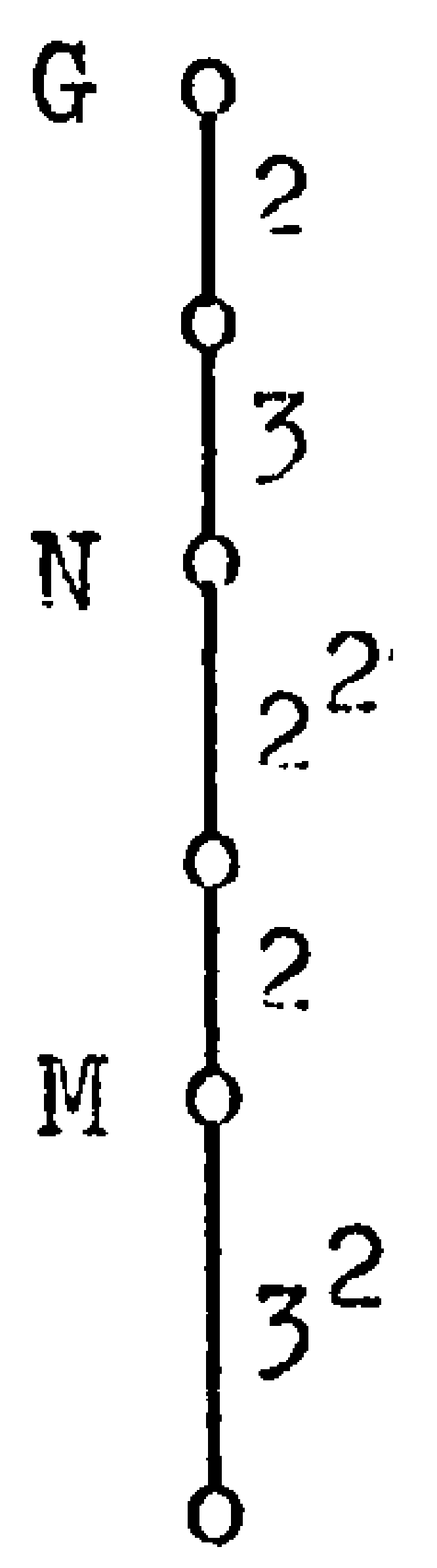

Therefore MA $\in \mathcal{F}_{3}$ but MB $\notin \mathcal{F}_{3}$ where $\mathcal{F}_{3}$ is the class of grouns with central 3-socle. (c.f. Fxample 2.3.2) 


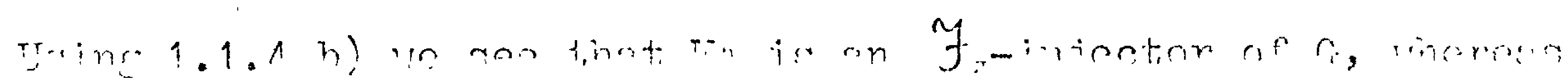

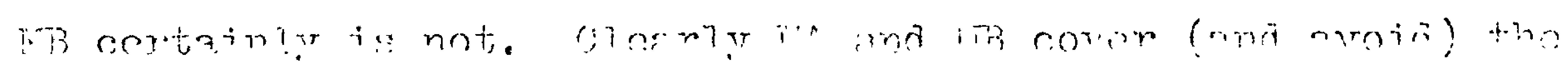

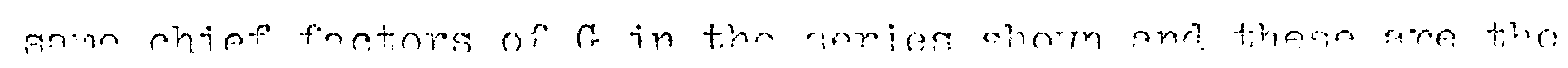

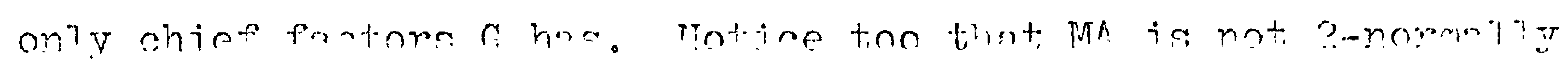
ambatractin G.

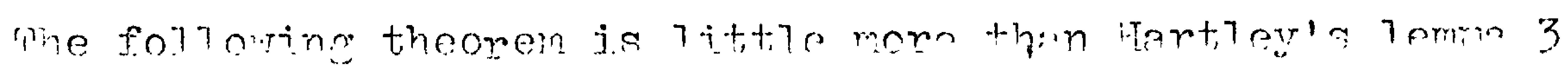

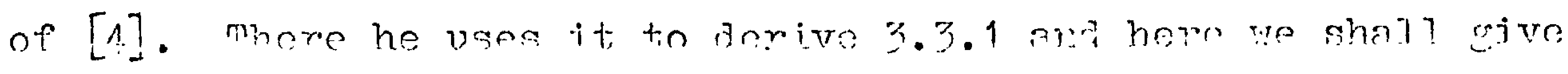
some furthar coroltarjes of this norerful result.

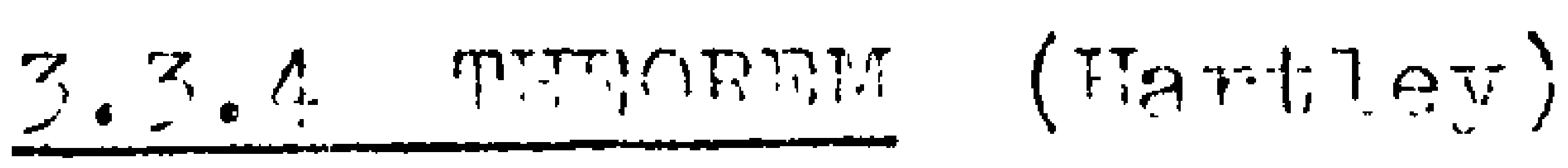

Sunnose $P$ is a p-subgroun of $r$ auch that

* $\quad \mathbb{4} \Delta \Rightarrow G=N_{G}(P \cap M) M$.

Them the folloring ame equivajent

a) P strongo G

b) $P$ nro G

c) $P \triangleleft G_{n}$ for some Srlor $n-s u b$ roun $G_{n}$ or $G$

d) $P$ centralines each p-chief frotor of $G$ which it avoids.

\section{PRONA}

phe imnlications $a ! \Rightarrow b) \Rightarrow c)$ are clear in viev of 3.1 .1 and 3.2.1 b). Sunmose c) holdas and $p<G_{p}$.

If $H / V$ is a $p$-chief factor of $G$ a.voided by $P$, then

$\left[\mathrm{P}, \mathrm{H} \cap \mathrm{G}_{\mathrm{p}}\right] \leqslant \mathrm{P} \cap \mathrm{H} \cap \mathrm{G}_{\mathrm{p}}=\mathrm{P} \cap \mathrm{H} \leqslant \mathrm{K}$. Butt $\mathrm{H}=\mathrm{K}\left(\mathrm{H} \cap \mathrm{G}_{\mathrm{p}}\right)$ a.nd so $P$ centrajizes $H / K$, therefore d) holds.

Observe that the condition * implies that $p$ covers or avoids cach n-chicf factor of $G$. For if $H / K$ is such a factor, we have $K(P \cap H) \triangleleft G$ by *, rhich yields the statement.

Now suppose d) holds. We show that, for any $X \triangleleft G$, the hynotheses * and d) carry over to $P \cap X$, and then deduce a) by induction on

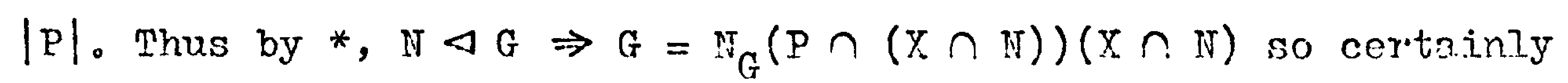
$G=N_{G}((P \cap X) \cap N) N$ and therefore $P \cap X$ satisfies condition * . 


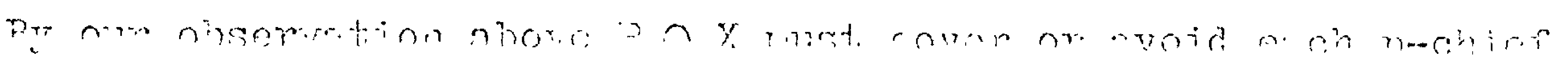

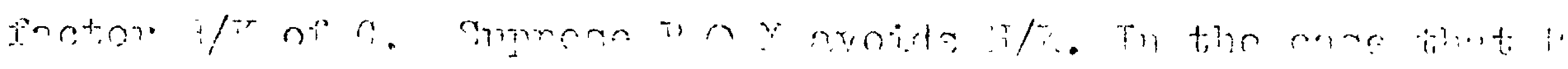

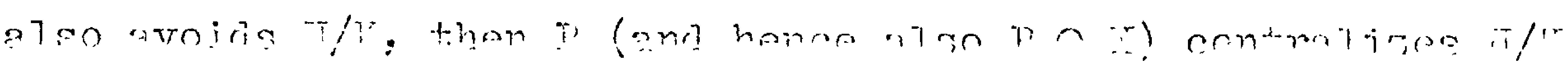

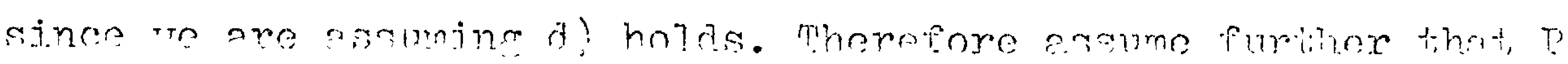

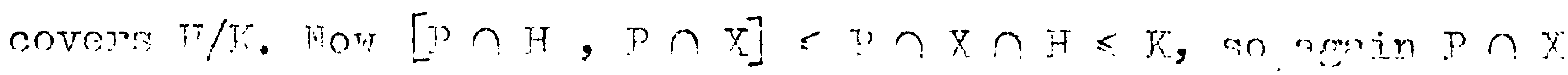

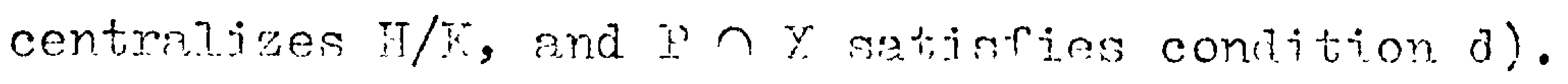

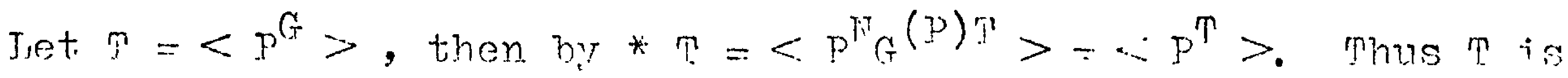

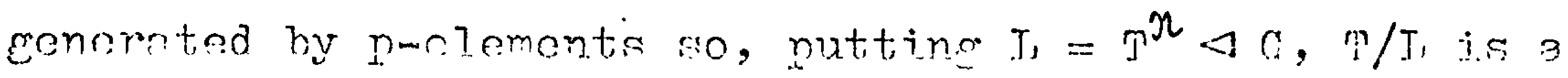
non-trivial n-cromp. Then since PT, sn $T=\left\langle p^{T}\right\rangle$, it foilotic that $\mathrm{PT}=\mathrm{T}$. So $P \cap T_{1}<\mathrm{P}$ and therefore by our induction hynothesis $P \cap I$ is a Sylow p-suhgroup of $\left\langle\left(P \cap I_{H}\right)^{G}\right\rangle=R$ sag.

$P$ avoirs all the n-chief factors of $(t$ between $R$ and $r$, so by supposition centiralizes them. Tt follows that $\mathrm{T}=\left\langle\mathrm{P}^{\mathrm{G}}\right\rangle$ centralizes them too, and hence that $I_{1} / R$ is n-nilnotent. But of course $T / R$ has no non-trivial p-quotient since $T^{\prime} / I_{1}$ is a $\mathrm{p}$-croup, so $T / R$ must be a $D^{\prime}-g r o u n$. This means $P \cap I$ j.s a Sylow $n$-suberoun of $\mathrm{T}$ and from $\mathrm{PT}=\mathrm{T}$ it follows that $\mathrm{P}$ is a sylow $\mathrm{p}$-subgroup of $\mathrm{T}$, so $P$ stropro $G$ and a) holds.

Subgroups $P$ rith property * arise very naturally in our theory. For sunpose $\mathcal{F}$ and $\mathcal{H}$ are arbitrary fittine classes and $G$ a groun. Let $P$ be $a$ sylow $p$-subgroun of an $\mathcal{H}$-injector $\forall$ of an $\mathcal{Y}$-injector $\mathrm{V}$ of G. Since Sylow subgrouns ana injecturs for a Fitting class. form characteristic conjugacy classes, it follows easily that $\mathrm{P}^{G}$ is a characteristic conjugacy class of $G$.

Eurthermore if $N \triangleleft \mathrm{F}$; then

$P \cap N$ is a sylow $p$-subgroup of an $\mathcal{H}$-injector $\forall \cap N$ of an F-injector $\mathrm{V} \cap \mathrm{N}$ of $\mathrm{N}$.

For certainly $V \cap \mathbb{N}$ is an $\mathcal{F}$-injector of $N$, but then $V \cap N \triangleleft V$ so $W \cap(V \cap N)=W \cap \mathbb{N}$ is an $\mathcal{H}$-injector of $V \cap N$. A.lso $W \cap N \triangleleft N$ so $P \cap(W \cap N)=P \cap N$ is a sylor $p$-suberoup of $\cap N$. 


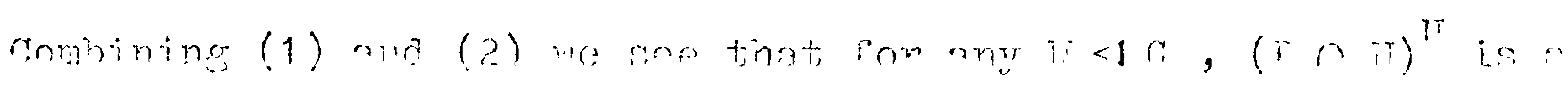

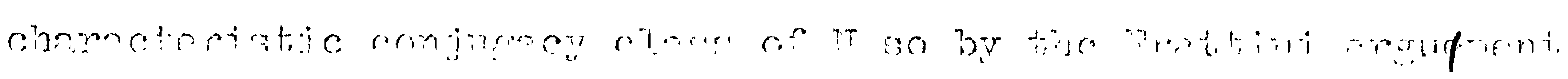

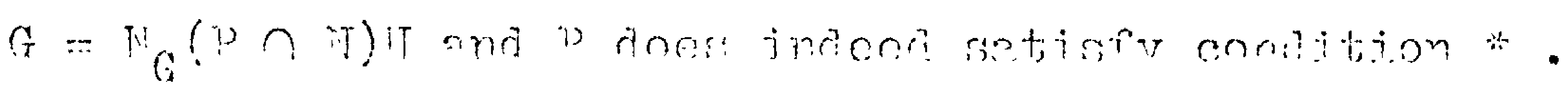

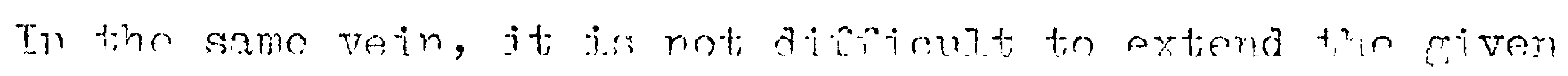

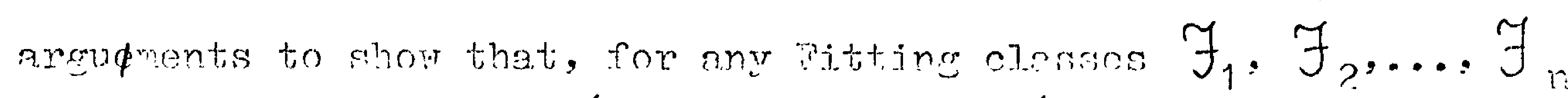
a. Sylor suberom of an $\mathcal{F}_{1}$-injector of an $\mathcal{F}_{2}$-injecion of an ... ... of an $\mathcal{F}_{n}$-iniecter of a rroun $G$ also setisties * . In frat

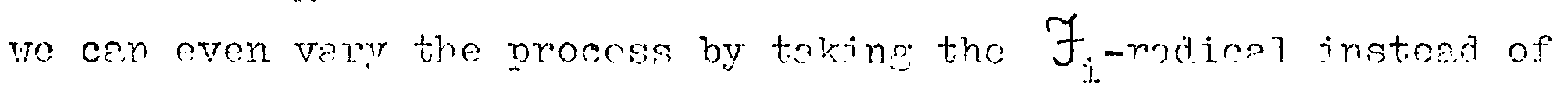
an $\mathcal{F}_{i}$-injector at the $i$ th stage, for various $i$, and still. obtain a sroun with nronenty * of courso the very case we hrove dealt with in (1) and (2) is itself an extonsion of the observation that, sylow subroup of an 7 -injector of a groun has pronerty * .

In view of this final remark, 3.3.4 immediately implies :

\subsubsection{Corollary}

If $P$ is a Sylow p-subgroin of an 7 -injector of a group $C$ and $P$ pro $G$ then $P$ stropro $G$.

\subsubsection{Corollary}

Iet $W$ be an $\mathcal{H}$-injector of an $\mathcal{F}$-injector $V$ of a group $G$ (where $\mathcal{F}$ and $\mathcal{H}$ are arbitrary pitting classes). If $V$ is $p$-normally embedded in $G$ and $W$ is $p$-normally embedded in $V$, then $V$ is p-normally embedded in $G$.

\section{Proof}

Let $W_{p} \leqslant V_{p} \leqslant G_{p}$ be Sylow subgroups of $H, V$, and $G$ respectively. By hypothesis $V_{p}$ is a Sylow p-subgroup of $\left\langle V_{p} G\right\rangle=K$ say. By our remarks above $<w_{p}^{G}>=\left\langle g_{p}^{K}>\right.$ since $W_{p}$ satisfies * in G. Also $V_{p}$ is a Sylow $p$-suberoun of the $\mathcal{H}$-injector $W \cap K$ of the $\mathcal{F}$-injector $V \cap K$ of $K$, so $\|_{p}$ satisfies * in the group $K$. BJ hypothesis $W_{p}$ stropro $V$ so in particular $W_{p}$ pro $V_{p}$ and henco 


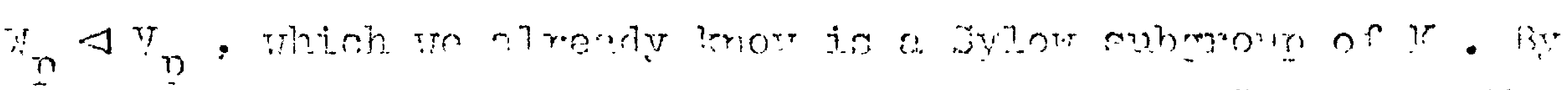

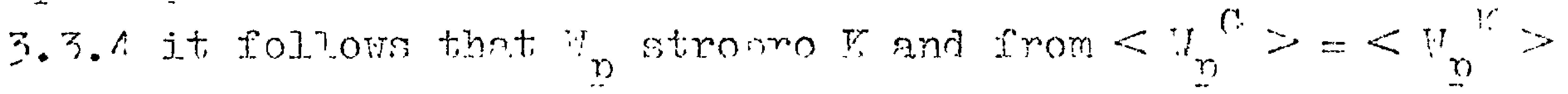
re downee trie rosizt.

\section{3 .7 cornz}

If $\mathcal{F}_{1}$ and $\mathcal{F}_{2}$ are both rittine rlasses for which the injertors of an arbjtrey grom are strongly pronomal, then the same is true ror $\mathcal{F}_{1} \mathcal{F}_{2}$

\section{Prnof}

Vie use the terminology of 2.6.4. Supnose $\mathrm{T}=\mathrm{H} \cap \mathrm{G}_{\mathrm{F}_{1}} \pi_{2}$, where is an $\mathcal{F}_{1}$-injector of $G$. By hymothesis stronro $G$, so using

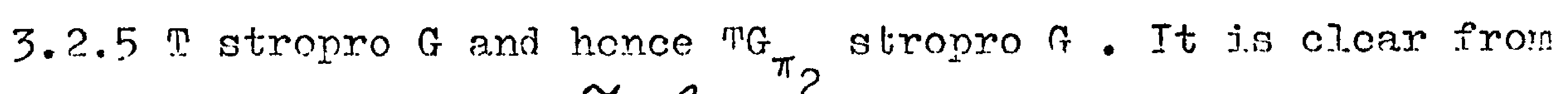
2.6 .4 that $G_{\pi_{2}}$ is an $\mathcal{F}_{1} S_{\pi_{2}}$-injector of $G$. By definition $v / \mathrm{r}$ j.s an $\mathcal{F}_{2}$-injector of $T_{\pi_{2}} / T$, so by hynothesis and $3.2 .1 \mathrm{~g}$ ) $V$ stronro $T G_{\pi_{2}}$. Certain $\mathrm{y} V$ is an $\mathcal{F}_{1} \mathcal{F}_{2}$-injector of $T G_{\pi_{2}}$. For each $p|| q \mid$, wo have established the hypotheses of 3.3 .6 , and its conclusion shors $V$ stronro $G$ as desired.

\section{3 .8 Remariks}

a) Supnose $\mathcal{F}$ is a Fitting class of characterjstic $\pi$ such trit

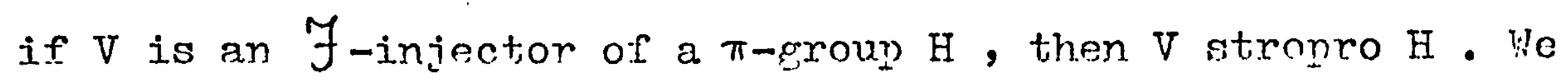
shor this actually imnlies that an $\mathcal{F}$-injector of an arbitrery groun $G$ is strongly oronormal in $G$. For as in 1.1 .6 ) an $\mathcal{F}$-injector $V$ of an $S_{\pi}$-injector $G_{\pi}$ of $G$ is an $\mathcal{F}$-injector of $G$. By supnosition $V$ stropro $G_{\pi}$ and clearly $G_{7}$ stropro $G$, therefore $V$ stropro $G$ by 3.3 .6 . For example, if $\mathcal{X}$ is a normal Fitting class and $\pi$ is a set of nrimes, an $\mathcal{X}_{\pi}$-injector of a $\pi$-groun $H$ is of course an $\mathcal{X}$-injector of $H$ and is therefore normat and hence strongly nronormal in $H$. sn it follows that an $\mathcal{X}_{\pi}$-injector of any group $G$ is strongly nronormal in $G$. 


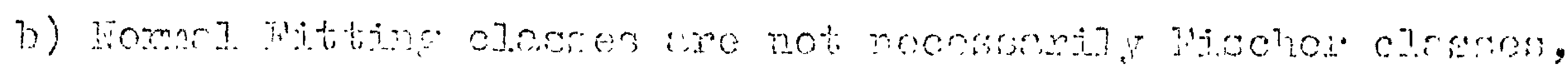

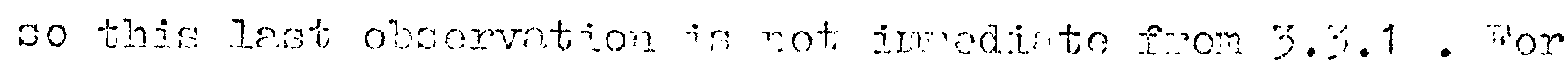

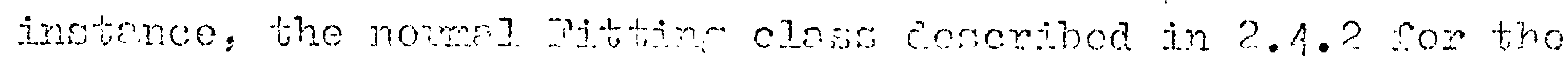
prime 3 conteins $G=\left[C_{3} \times C_{3}\right] C_{2}$ (whore onch involution inverso each 3 -elemont) but not the subroun $i_{3}$ of 4 . Thover this subgrong is an extension of a nomat subcroun of 4 by a 2-croup, and therefore 7.jes in exch Fischer clans to which e bolongs.

c) The strong pronormality of the injectors for a Tischer clese is exploited in rork of vaken [16]. There he shows that in each group $G$ an injector for a Fischer class pormutos with a prefretbini subcroup, and ho estublishes covering and avoidance properties of their preduct. 


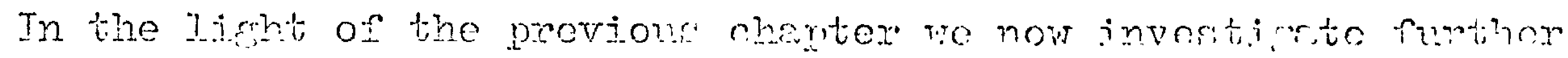
the closs $\mathcal{F}^{\pi}$ with a vier to subetentietine remerl: 2.6.2 c) . Fxtonling our terminolocy we shel]. cell a. Fittine class $\mathcal{F}$ Stroncly pronomel if the $\mathcal{F}$-jnjectors of ccch croun $G$ are stronsly proromel in 6 . Further ve say 7 is permutable if on F-jinjector $V$ of an erbitrers spoun $G$ permutes vith eech mumer of ony sylow system $\Sigma$ of $G$ which reduces into $V$. We wite $V \perp \Sigma$ in this case. (By remaris $3.1 .7 \mathrm{c}$ ) this holds if, for each $\pi, v$ pernutes with some Hell $\pi$-submroup of G.)

In the sccond section of this chepter ro exminc an interesting Ionmo of Fischer, and establish conditions equivalont to the permutability of a Fitting class, involving systen normalizors.

\subsection{Permutable Fitting classes}

\subsubsection{Proposition}

If $\mathcal{F}$ is strongly pronormal (for example a Fischer class or normal Fitting class) then $\mathcal{F}$ is permutable.

\section{Proof}

Suppose the sylow system $\Sigma$ of $G$ reduces into an $\mathcal{F}$-injector $V$ of $G$. Certainly each $G_{\pi} \in \Sigma$ is strongly pronormal in $G$ and clearly $\Sigma \searrow G_{\pi}$. So if $V$ stropro $\approx$, then by 3.2.j $V \perp G$ and the result follows.

\subsubsection{Proposition}

Suppose $\mathcal{F}$ is a permitable Fitting class and $G$ a group with Sylor system $\Sigma$. Let $V$ be the unique $\mathcal{F}$-injector of $G$ into which $\Sigma$ reduces. Then
a) $V G \pi^{\prime}$ is an $\mathcal{F}^{\pi}$-injector of $G$ and
$\mathcal{F}^{\pi}$ is permutable.
b) $V G_{\pi_{1}}, \cap V G_{\pi_{2}}{ }^{\prime}=V G\left(\pi_{1} \cap \pi_{2}\right)$, is an and $\mathcal{F}^{\pi_{1} \cup \pi_{2}}=\mathcal{F}^{\pi_{1}} \cap \mathcal{F}^{\pi_{2}}$. $\mathcal{F}^{\pi_{1} \cup \pi_{2}}$-injector of $G$ 


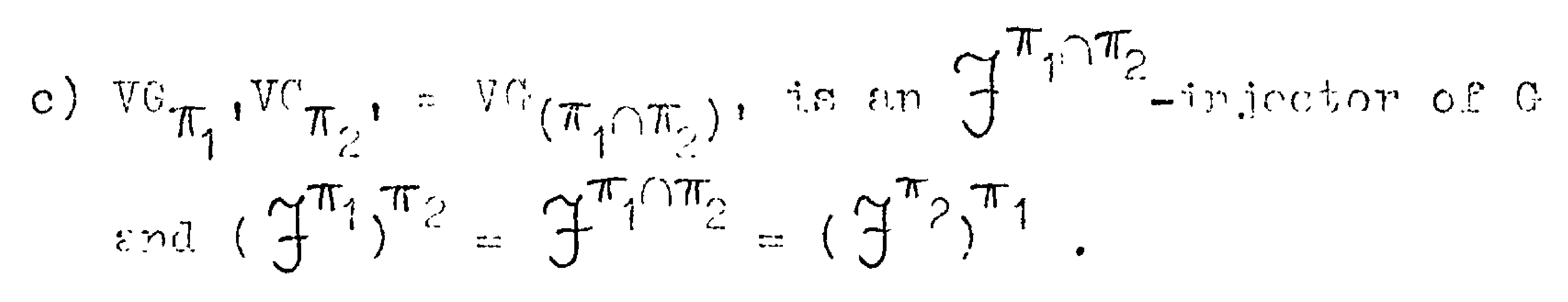

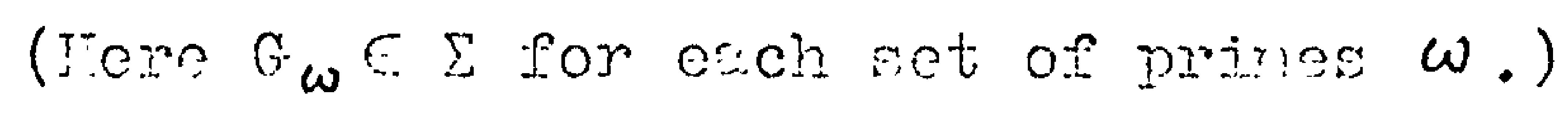

Pront

a) The first part is 2.6.3. By $3.1 .5 \mathrm{\Sigma} \supset \mathrm{VG}_{\pi^{\prime}}$, so this is tive uniaue $\mathcal{F}^{\pi}$-injector of $G$ into which $\Sigma$ reduccs. Clearly

$\left(V G_{\pi^{\prime}}\right) G_{\omega}=V G_{\pi v \omega}$, which is a subrroup of $G$ by the permutroilitiy of $\mathcal{F}$, so we heve shom $\mathcal{F}^{\pi}$ is permutabic.

b) $\left(\pi \pi_{1} \cap \pi_{2}^{\prime}\right)=\left(\pi_{1} \cup \pi_{2}\right)$ ' so certein $l y V G_{\pi_{1}}, \cap V G_{7 \pi_{2}} \geq V G_{\left(\pi_{1} \cup \pi_{2}\right)}$ '. On the other hand it is exsily seen that $\left|V G\left(\pi_{1} \cup \pi_{2}\right) \cdot\right|=$ $\operatorname{ecd}\left(\left|V G_{\pi_{1}},\right|,\left|V G_{\pi_{2}},\right|\right)$, so equality of the subgroups follows. Bearing a) in mind the rest is obvious.

c) $\left(\pi_{1} \cup \pi_{2}^{\prime}\right)=\left(\pi_{1} \cap \pi_{2}\right)$ ' so by a) $V G_{\pi_{1}}, V G_{\pi_{2}}{ }^{\prime}=V G\left(\pi_{1} \cap \pi_{2}\right)$, is an $\mathcal{F}^{\pi_{1} \cap \pi_{2}}$-injector of $G$. Also by a) $\mathcal{F}^{\pi_{1}}$ is pemutable so the unique $\left(\mathcal{f}^{\pi_{1}}\right)^{\pi_{2}}$-injector of $\mathrm{G}$ into which $\Sigma$ reduces $=$ (the unique $\mathcal{F}^{\pi_{1}}$-injector of $G$ into which $\sum$ reduces $) \sigma_{\pi_{2}}$ =

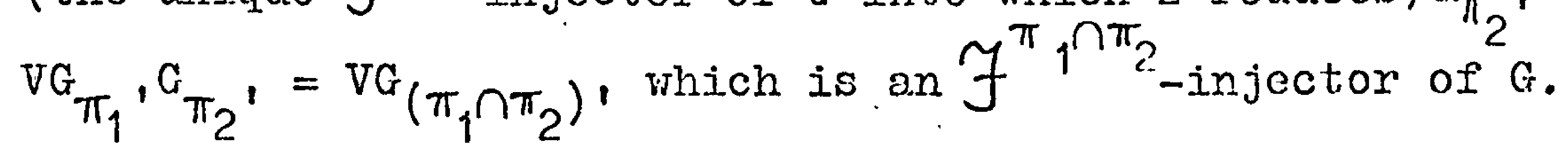
Coincidence of injectors in all groups clearly implies the coincidence of the classes, so by symmetry the whole statement follows.

\subsubsection{Proposition}

$\mathcal{F}_{1}$ and $\mathcal{F}_{2}$ are permutable Fitting classes implies $\mathcal{F}=\mathcal{F}_{1} \mathcal{F}_{2}$ is permutable.

\section{Proof}

Let $\Sigma$ be a fixed Sylow system of the group $G$. Our proof is another illustration of the principle mentioned in $3.1 .7 \mathrm{c}$ ). Let $T$ be the unique $\mathcal{F}_{1}$-injector of $I=G_{\eta_{1}} \pi_{2}$ into which $\Sigma$ reduces. By 3.1.10 and the Frattini argupment $T$ is normalized by $G_{\pi_{2}} \in \Sigma$. Wheorom 2.6.4 said that if $V / T$ is an $\mathcal{F}_{2}$-injector of $T G_{\pi_{2}} / T^{2}$, then 


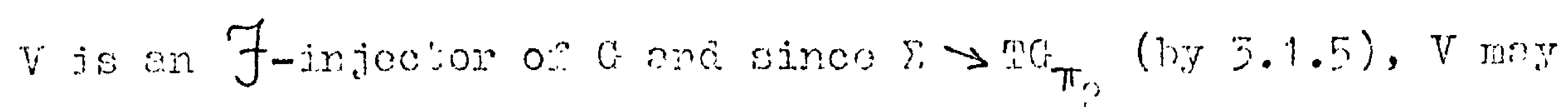
be then to bo the uniauo $\mathcal{F}$-injector of $\mathrm{G}$ intio mich $s$ redure

For any $\pi$ ve mut whow thet $V \perp c_{\pi}$ C. $\Sigma$. By $2.6 .5 \mathrm{Tr} / \mathrm{K}$ is on $\mathcal{F}_{2}$-injector of $c / K$ end tho system $\dddot{H}_{i}=\left\{\ldots, a_{\pi} K / K_{n}, \ldots\right\}$ of $\mathrm{c} / \pi$ reduces into $\mathrm{Vr} / \mathrm{K}$, so by the ascumod pormutebilitity of $\mathcal{F}_{2}$ $\mathrm{VE} / \mathrm{K} \perp \mathrm{C}_{\pi} \mathrm{K} / \mathrm{K}$, thet is $\mathrm{VYc}_{\pi}$ is a subrroup or $\mathrm{G} \cdot \mathcal{F}_{1}$ i.s a.so perruteble by hypotissis so $V \cap I=T \perp G_{\pi} \cap T=X_{\pi}$ say. How by 3.1.6 $\pi$ pro $G$ and has $\left(\pi_{2} \cup \pi\right)$ '-index in $T$, so by the Trattini

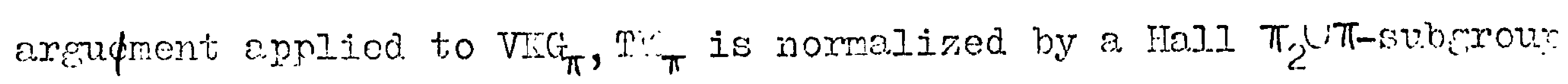
of $\mathrm{VKG}_{\pi}$, which by 3.1 .10 anain may be taken to be $\mathrm{VKG}_{\pi} \cap G_{\pi_{2}}^{G} \pi$ since $\Sigma \longrightarrow \mathrm{VKG}_{\pi}$. By the moduler leiv

$$
\begin{aligned}
& \mathrm{TI}_{\pi}\left(\mathrm{VKK}_{\pi} \cap \mathrm{G}_{\pi_{2}} \mathrm{G}_{\pi}\right)=\mathrm{VIKG}_{\pi} \cap \mathrm{TG} \mathrm{G}_{2} \mathrm{G}_{\pi} \\
& =V G_{\pi}\left(F \cap P G_{\pi_{2}} G_{\pi}\right) \\
& =V G_{\pi} T\left(K \cap G_{\pi_{2}} G_{\pi}\right) \\
& =V G_{\pi} T\left(K \cap G_{\pi_{2}}\right)\left(I \cap \cap G_{\pi}\right) \\
& =\mathrm{VG}_{\pi} \quad \text { (since } \mathrm{K} \cap \mathrm{G}_{\pi_{2}} \leqslant \mathrm{~T} \leqslant \mathrm{~V} \text { ) }
\end{aligned}
$$

\subsubsection{Remarks}

a) 2.6 .1 shows that for arbitrary $\mathcal{F}$, an $\mathcal{F}^{p}$-injector $v$ of a group. $G$ has p-pover index in $G$, so by an obvious order argufment $V \perp \sum$ if $\Sigma \backslash V$ (each product is either $V$ or $G$ ). Thus $\mathcal{F}^{p}$ is a permutable class. With this in mind we can use example 3.3 .3 to deny the converse 4.1 .1 , at the same time shoving that the injectors for a permutable Fitting class are not necesserily characterized by their covering and avoidance properties. For (in the notation of the example) $\mathrm{In}$ is an $\left(\mathcal{F}_{3}\right)^{\{2\}}$-injector of , $G$ by 1.1 .4 b) while $M B$ is not, and as we saw MA is not 2-normally embedded.

b) $\mathcal{F}^{\pi}$ is clearly the set of all those groups which are the product of an $\mathcal{F}$-injector and a Hall $\pi^{\prime}$-subgroup, and we have just seen that when $\mathcal{F}$ is strongly pronormal, an $\mathcal{F}^{\pi}$-injector of 


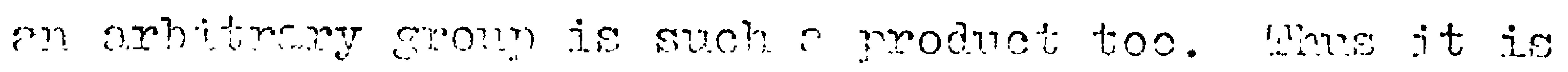

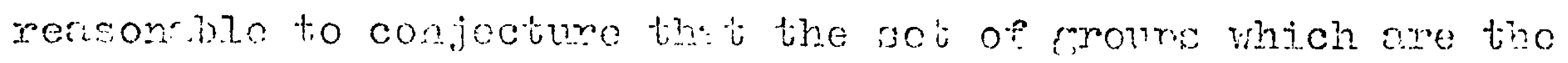

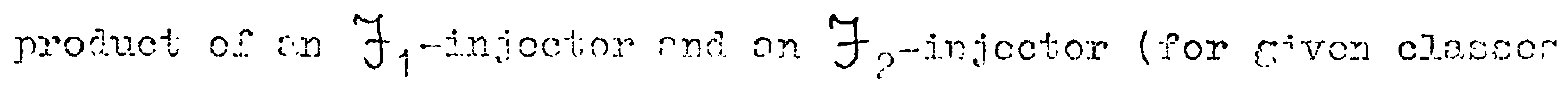
$\mathcal{F}_{1}$ and $\mathcal{F}_{2}$, rom a Fittine class $X$ sa.y, and hoperulty an X-injoction of any groun G js a.1so such s product. (thy 3.2.6 we miey ensure thet this product alvays existis by taking $f_{1}$ and $\mathcal{F}_{2}$ to be stronsily pronomel classes.) our cxpectations are strengthenod by the elegint dual result (for schunch classes) due to Bjessenohl (theorem $\overrightarrow{7} \cdot 3.8$ of [7]). However the following excmple shows that this 'join' $\mathcal{X}$ of pitting classes $\mathcal{F}_{1}$ and $\mathcal{F}_{2}$ need not be Fittins. Let $\mathcal{F}_{1}$ be the nomal class doscribed in 2.4.2 for the prime 3 and $\mathcal{F}_{2}$ that for the prine 5 . Put $G=D_{6} \times D_{10}$, then $G_{F_{1}}=C_{3} \times D_{10}$ and $G_{F_{2}}=D_{6} \times C_{5}$, so $G=G_{F_{1}} G_{F_{2}}$. But $G$ has a normal subrroup $\mathrm{I} \cong\left[C_{3} \times C_{5}\right] C_{2}$ (inere each involution inverts each $2^{\prime}$-element) and $N_{\mathcal{F}_{1}}=C_{3} \times C_{5}=N_{F_{2}}$, so $N \neq I_{F_{1}} N_{2}$.

c) It seems to be very difficult in general to decide whether a non-stroigly pronomal Fitting class is permutable or not and in particular we have been uneble to determine whether the class $\mathcal{F}_{\mathrm{p}}$ of groups with central p-socle enjoys this property, though we suspect it does not. Thus to show that there exist classes which are not permuteble we fall back on the rork of Dark, and our example is a simple ammendment of his.

Dark constructs (by methods akin to 1.2 .4 ) a group $A B C D=J$ say with a unique chief series, in which each of the subgroups $A, B, C$, and $D$ covers the chief factor shown, and A normalizes $B, C$, and $D, B$ normalizes $C$ and $D$, and $C$ normalizes $D$. He nov examine $J$ in more detail.

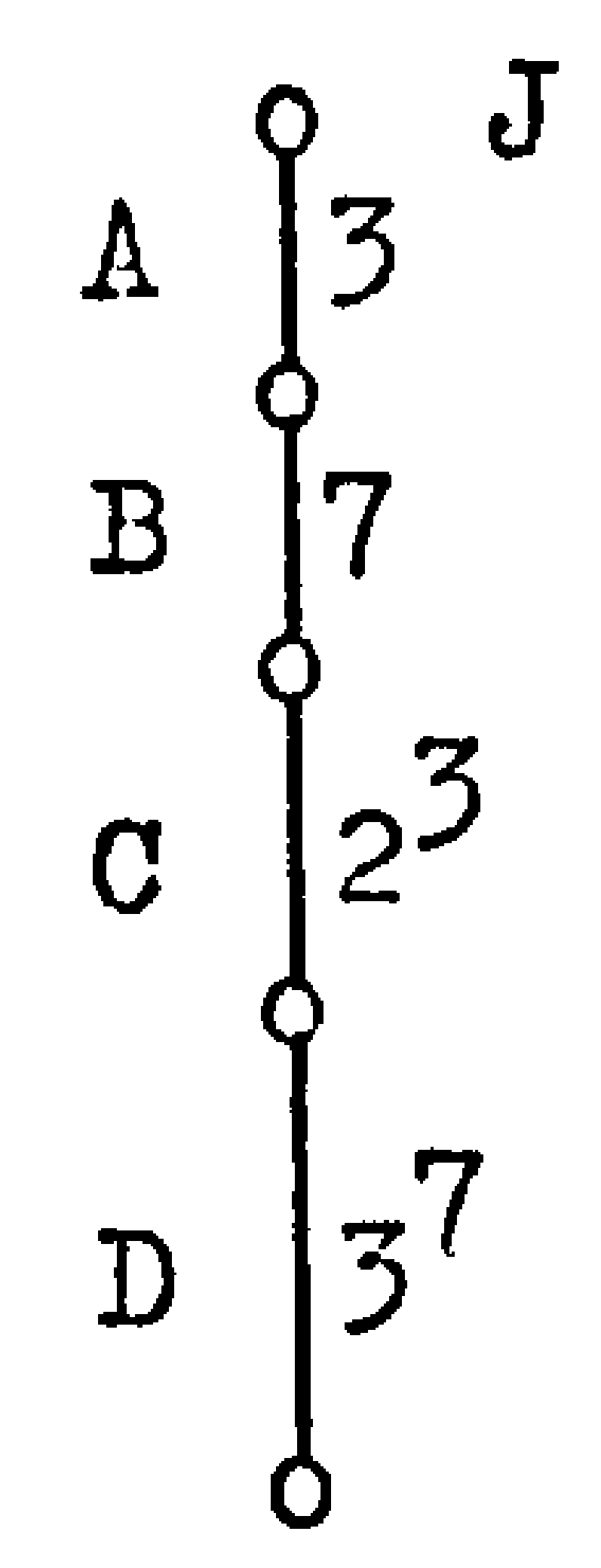
(Indeed the argupments apply to any group with the stated properties of $\mathrm{J}$. ) 
In Dortra notation, $A=\left\langle a^{\prime}>\right.$. B does not centronize $D$, 'nd since 3 hes order 6 mod 7 it follors thet is $=[D, D] \times C_{p}(s)$ is the decorposition of i) into en irreducible componont of nin $3^{6}$ and a trivial component of order 3 , under the action of $\mathrm{B}$. Let $C_{D}(B)=\langle d\rangle$. $A$ normelizes $B$ and $D$, so it nornelizes bould $[D, B]$ and $\langle a\rangle$, hence $a$ and a commute. Iset $a=a^{\prime} d$.

We show $<$ a > normalizos no Sylor 2-suberoup of $\mathrm{J}$.

Suppose it does, then wo mey assuno a nomalizes $C^{d}$ where $d_{0} \in D$. Then $a^{d_{0}^{-1}}=\left(a^{\prime} d\right)^{d_{0}^{-1}}$ normelizes $c$. But $a^{\prime}$ normalizes $c$, so

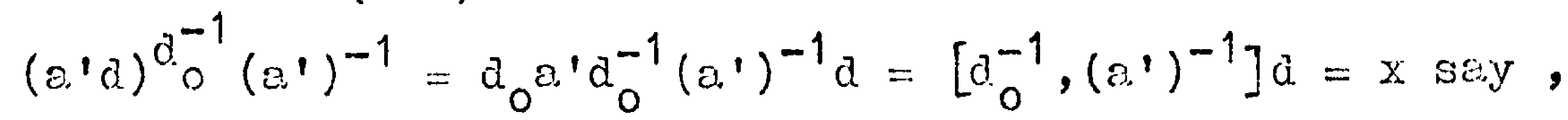
nomelizes $C$ too. Now $\Lambda$ centrajizes $D /[D, B]$, so $\left[d_{0}^{-1},\left(a^{\prime}\right)^{-1}\right] \in[D, B]$ and hence $x \neq 1$. Then $[C, x] \leqslant C \cap D$, so $x$ is a non-trivial fixed point or $C$ in $D$. How $C_{D}(C)$ is normajized by $A, B, C$ and $D$, that $j$.s by $J$, and $D$ is a minimal normal subgroup of $J$, so $C$ contralizes $D$, a contradiction. Notice that $B<a^{\prime}>$ and $B<a>$ are not conjugate in $J$. For if they are, it follows from Sylow's theorem that $\left\langle a^{\prime}>\right.$ and $\langle a\rangle$ are conjugate in $J$ and hence that $<a>$ normalizes $a$ Sylow 2-subgroup of $\mathrm{J}$, against (1) .

(Notice too that we have filled in some of the detaila of 1.1.6.)

Dark then constructs a metabelian group $E$ of order $19^{777}$ on which J acts, and writes G for the corresponding split extension. His Fitting class $\mathcal{X}$ is constructed from the group $H=$ $<a>B E$ (the construction ve imitated in chapter 2) and satisfies $S_{3} \leq \mathcal{X}, \mathrm{H} \in \mathcal{X}$, $D E, \triangle B C E \notin \mathcal{X}$. (Notice that $J=A B C D$ is a primitive group, so any complement in $G$ of 'the chicf factor $D$ ' is a conjugate of ABCE in G.)

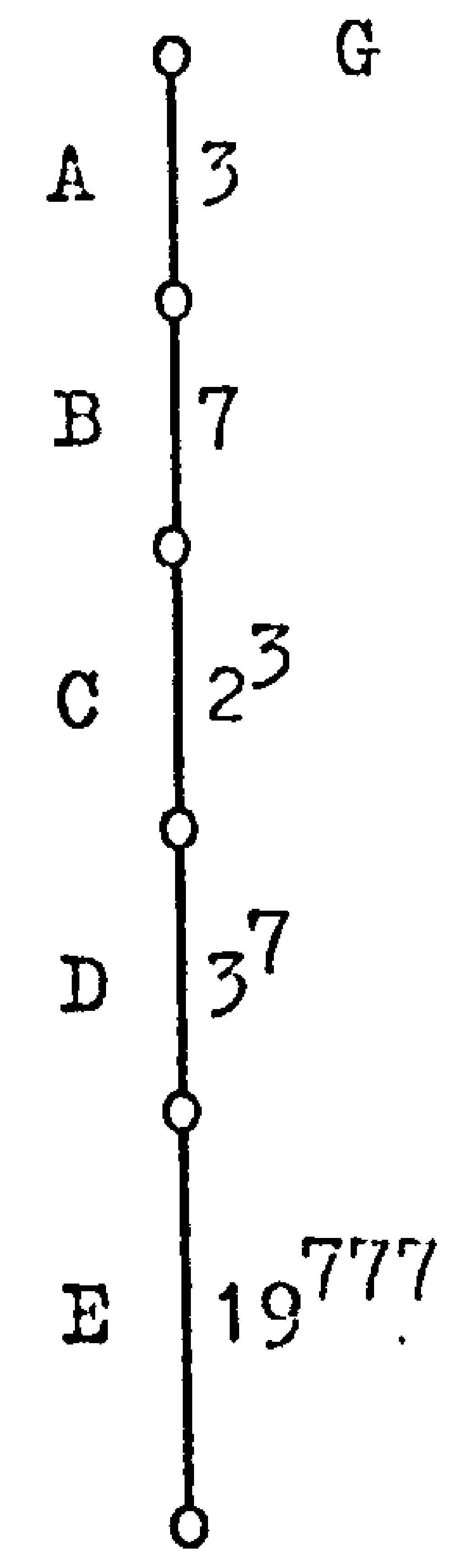




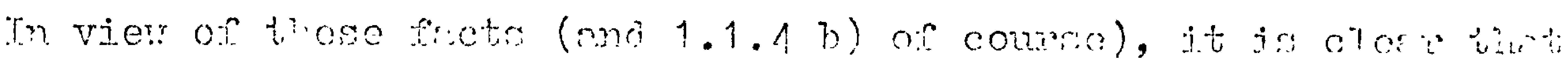

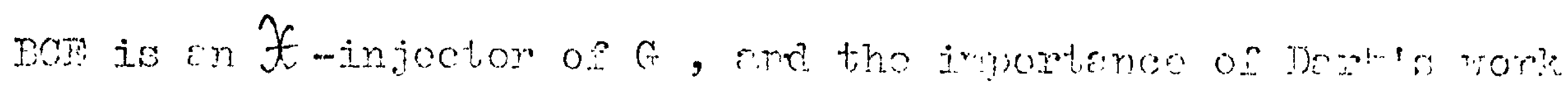

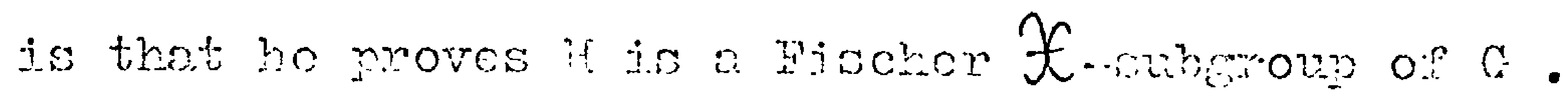

Irow let $F=f \cap S_{19} S_{7} S_{3}$. Furthor arplication of 1.1 .4 . )

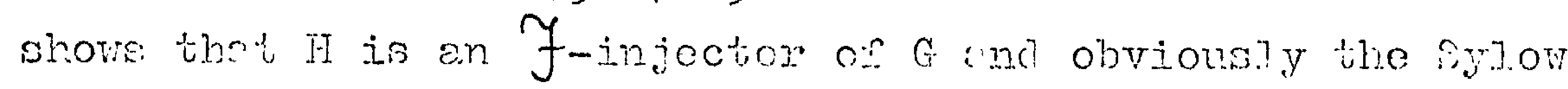

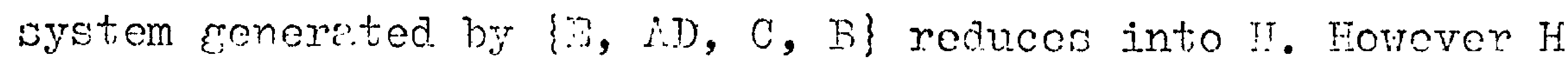
connot permute vith os since by (1) above, $<a>$ fails to nomalize $c$. Thus $\mathcal{F}$ is not a permutalo pitting class. (Observe that $A B E \Phi \mathcal{F}$, for if not $\Lambda B D$ is (by 1.1 .4 b)) an $\mathcal{F}$-injector of $G$ and therefore conjurete to $\mathrm{H}$ arainst (2). So in particner $\left.\mathrm{A}_{\mathrm{BE}}=\left\langle\mathrm{a}^{\prime}\right\rangle \mathrm{BR} \neq<\mathrm{a}\right\rangle \mathrm{BE}=\mathrm{H}$.)

d) Nore Eymnastics with Darl''s groun show that $\left(\mathcal{f}^{\pi_{1}}\right)^{\pi_{2}}=\left(\mathcal{f}^{\pi_{2}}\right)^{\pi_{1}}$ can be folso when $\mathcal{F}$ is not permutable. This time ve let $\mathcal{F}=\mathcal{K} \cap S_{19} S_{7} S_{5} S_{3}$ and consider $G 2 c_{5}=G$ * say, where $\mathcal{K}$ and $G$ are as above. Let $B^{*}=B \times B \times B \times B \times B$ etc. Since 3 has order $4 \bmod 5$, and with the aid of the lemma in [17], it follows that the factors of $G^{*}$ above $E^{*}$ as shorn, are chief factörs. Romembering that injectors cover or avoid chief factors it is not difficult to verify that $\mathrm{E}^{*} \mathrm{C}^{*} \mathrm{~B}^{*} \mathrm{C}_{5}$ is an $\mathcal{f}\{3,5\}$-injector $0: G^{*}$ and hence $G^{*} \in(\mathcal{F}\{3,5\},\{2,5\}$. Also $E^{*} D^{*} B^{*} A^{*}$ is an $\mathcal{J}^{\{2,5\}}$-injector of $G^{*}$ which fails to cover the 5-chief factor, so $G^{*} \notin(\mathcal{F}\{2,5\},\{3,5\}$. '

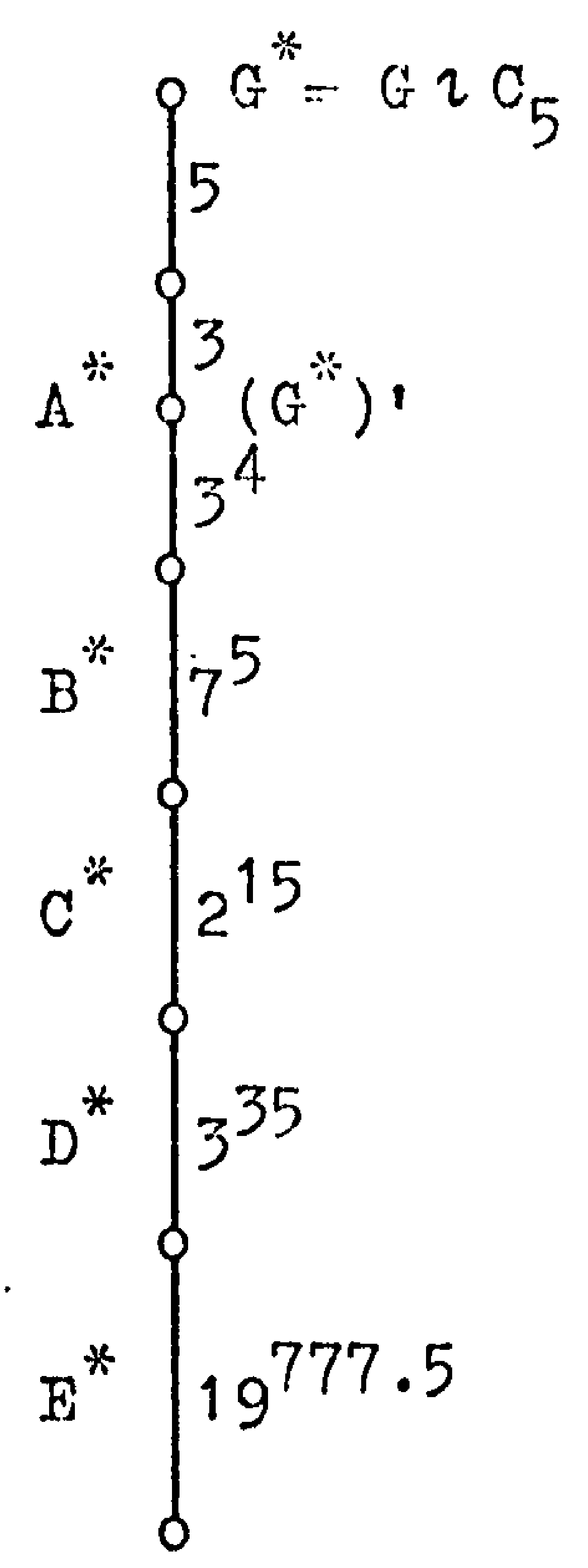

e) Remark 3.3 .8 a) said that a Fittine class of characteristic $\pi$ is strongly pronomal if it is 'strongly pronormal in $\delta_{\pi}$ '. We conjecture that similarly, such a Pitting class is permutable 
ir it is pommtoble in $S_{\pi}$. Tinforturaten the conivelences

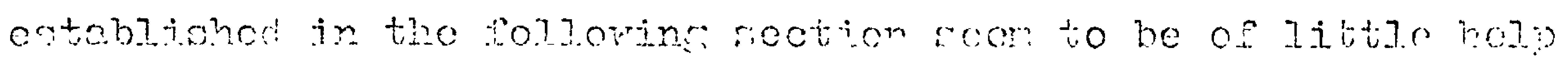
in proving thin.

\section{4.? Fischor's Temro and conditions ecuivolont to pemutehisity}

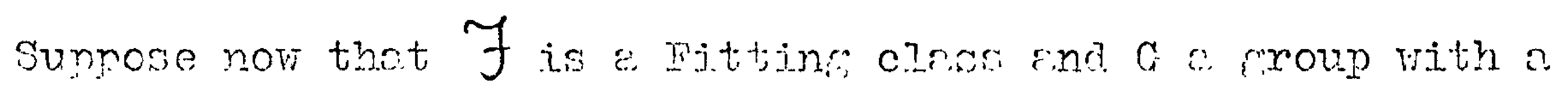
normal subrroup $N$ such that $G / N \in \mathcal{N}$, and $N y=F$ say, is

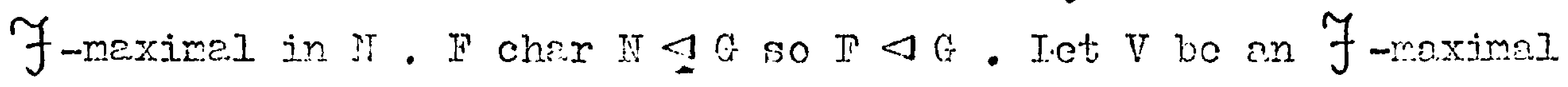
sipgroup of $G$ contajning: $F$.

Br 1.1.4 a) $V$ is an 7 -injector of $G$ and therefore $V$ pro $G$.

As a corollary of the nroof of Ilart]cy's lemma 1.1 .3 we have $V=(F C)_{y}$ for some Cartier subgroun $C$ of $G$.

our first aim is a now proof of the following sharper varsion of (2), known to Fischer, whose lons argupment appears in [2].

\subsubsection{THEORPA (Fischer) \\ $V=(F D)_{y}$ for some system normelizer $D$ of $G$. (Specifically} this holus for the normalizer of any system which reduces into V.) PROOF

We need the folloving lemma, inspired by Fischer's approach.

\subsubsection{Iemma.}

Let $\mathrm{P}$ be a pronormal p-subgroup of a group $H$ which complemonts $\mathrm{K} \triangleleft \mathrm{H}$. Then $\mathrm{P}$ normalizes each $\mathrm{p}$-complement of $\mathrm{K}$.

Proof

If $J \triangleleft H$ and $J \leqslant I$, then $P J / J$ is a pronormal (by 3.1 .1 c)) complement of $\mathrm{K} / \mathrm{J} \triangleleft \mathrm{H} / \mathrm{J}$, so the hypotheses carry over to the quotient $\mathrm{H} / \mathrm{J}$, and we argue by induction on $|\mathrm{H}|$. If $\mathrm{o}_{\mathrm{p}^{\prime}}(\mathrm{K}) \neq 1$, then by the induction hypothesis, $P \mathrm{P}_{\mathrm{p}^{\prime}}(\mathrm{K}) / \mathrm{O}_{\mathrm{p}^{\prime}}(\mathrm{K})$ normalizes cach $\mathrm{p}$-complement of $\mathrm{K} / \mathrm{O}_{\mathrm{p}^{\prime}}(K)$, which yields the desired conclusion. If $o_{p^{\prime}}(K) \neq 1$, then $1 \neq o_{\underline{p}}(K)=K_{x}$ the Fitting 


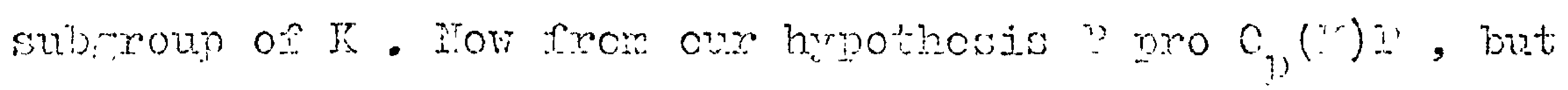

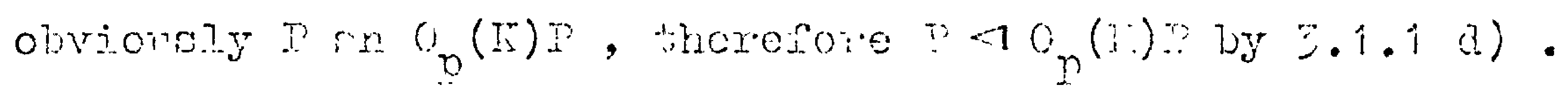

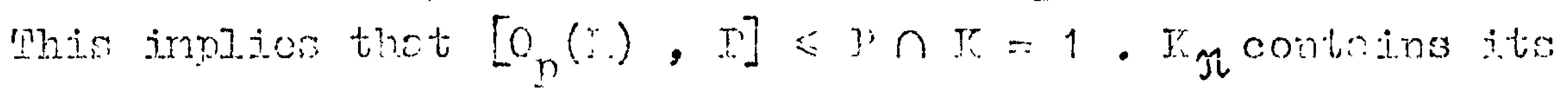
centrazizer in in so jt follone thet

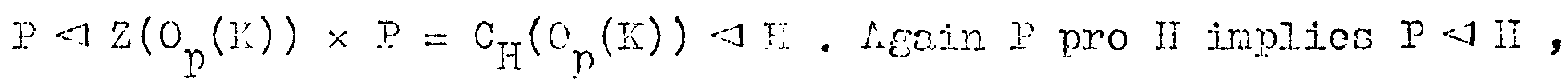
so $I I=I \times P$ ond the result obvjously holds in this case.

Suppose the Sylow sortem $\Sigma=\left\{G_{p}, \ldots, G_{\pi}, \ldots, G_{p}, \ldots\right\}$ of $G$ reduces into $V$. Whenover $\Sigma \rightarrow m \leqslant G$ we vrite $T_{\pi}=r \cap G_{\pi}$ for each $G_{\pi} \in \Sigma$. Let $\bar{G}=G / \Gamma$, then $\bar{\Sigma}$ (the images of the members of $\Sigma$ under the notural epimorphism) reduces into $\bar{V}$, and $\bar{G}_{p} \cap \bar{V}=\bar{G}_{p} \cap \bar{V}=\bar{V}_{p}$ etc. is easily shom. Nov $G / \mathbb{N} \in \mathcal{L}$, so

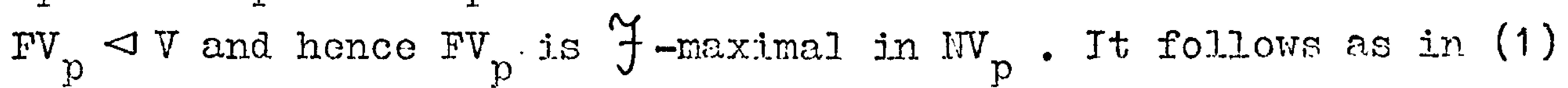
that $F V_{p}$ is an $\mathcal{F}_{\text {-injector of }} \mathrm{WV}_{\mathrm{p}}$, therefore $\mathrm{FV}_{\mathrm{p}}$ pro $\mathrm{NV}_{\mathrm{p}}$. Thus $\bar{V}_{p}$ is a pronormal. p-subsroun of $\overline{I V}_{p}$ complementinc $\bar{N}$, so by 4.2.2 $\bar{V}_{p}$ normalizes each $p$-comploment of $\bar{N}$, and in particular normalizes $\bar{F}_{p^{\prime}}$. Now $\Sigma>V$, so $\bar{V}_{p^{\prime}} \leqslant \bar{G}_{p^{\prime}}$ and hence $\bar{V}_{p^{\prime}}$ nomalizes $\bar{I} \cap \bar{G}_{p^{\prime}}=\overline{I T}_{p^{\prime}}$. We have therciore shom that $\bar{V} \leqslant H_{\bar{G}}(\bar{\Sigma} \cap \overline{\mathrm{I}})=\mathrm{E} / \mathrm{F}$ say, a relative system normalizer of $\overline{\mathrm{IT}}$ in $\bar{G}$. First. supposic that $E=G$. Clearly this holds iff $\bar{N}=I / F \in X$, so in this case $\mathrm{G} / \mathrm{F} \in \mathrm{M}^{2}$. A rosult of long standing due to Carter states that Cartor subgroups and systom normalizers are the same thing ir $\bigwedge^{2}$-Eroups, so by the homomorphism invariance of both of these classes of subgroups, the product FC coincides vith $F D$, where $D$ is any system nomalizer of $G$ Iying in $C$, and then the statcrnent of the theorom follows from (2).

Wo shall now assume that $\mathrm{E}<\mathrm{G}$, and suppose inductively that the theorem holds for groups of order less than $|G|$. certainly $V$ is an $f$-maximal subgroup of $E$ containing $F$, and $F$ is 7 -maximal and normal in $N \cap E$. Moreover If $R \Delta E$ and $\mathrm{E} / \mathrm{N} \cap \mathrm{E} \cong \mathrm{NE} / \mathrm{N} \leqslant \mathrm{G} / \mathrm{N} \in \mathcal{M}$. Thus the conditions of the theoren 


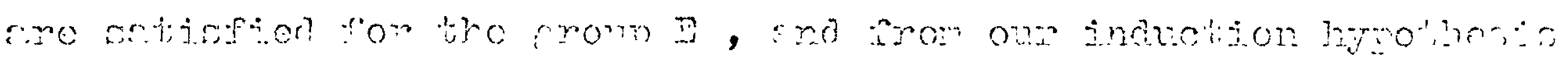

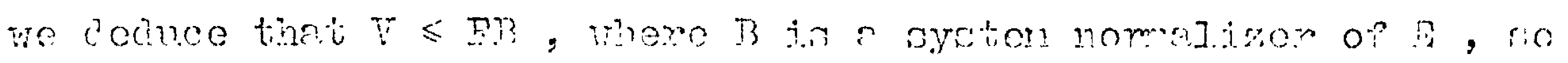

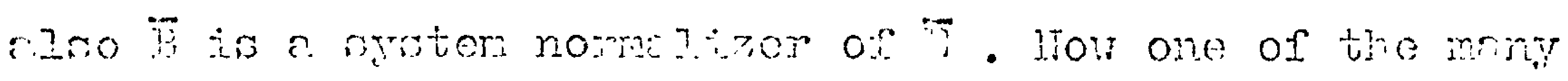

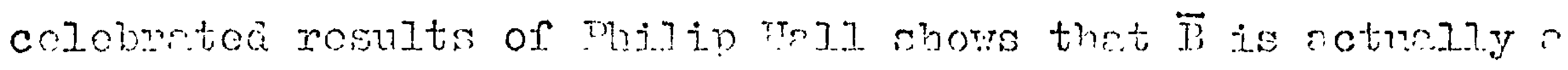

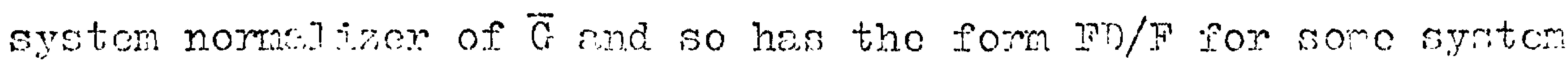

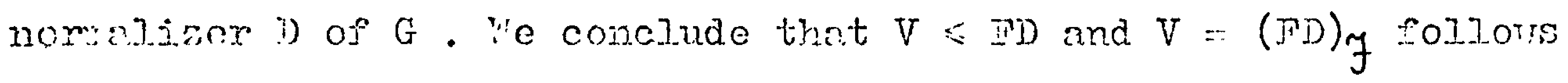
at once.

Let $D^{*}=\mathrm{N}_{G}(\Sigma)$, then it is well-1mom that $\Sigma \longrightarrow D^{*}$. Using 3.1 .5 we sec $\left.s>(E I)^{*}\right)_{\mathcal{F}}$, but of corrso $\left(R D^{*}\right)_{\mathcal{F}}$ is a conjugete of $V$ which is pronomal in $c$, so by $3.1 .3 \mathrm{~V}=\left(\mathrm{F}^{*}\right)_{\mathcal{F}}$ end the proos is complete.

Suppose nert that :

$\mathcal{F}$ is a sititing, class and $G$ a group rith o. Sylow system $z=$ $*\left\{G_{p}, \ldots, G_{\pi}, \ldots, G_{p}, \ldots\right\}$ reducing into the $\mathcal{F}$-injector $V$ of $\hat{i}$, and $D=\mathbb{N}_{G}(\Sigma)$.

If $N \triangleleft G$ with $G / N \in M$, then wo have just secn that $V \cap N \varangle G$ implics $V \leqslant(V \cap N) D$. In general we only lnov that $V \cap \mathbb{N}$ pro $G$ $(1.1 .7 \mathrm{a}))$, so certainly $D$ normalizes $V \cap N$ by 3.1 .5 and 3.1 .10 . We ask under what circumstances is the inclusion $V \leqslant(V \cap N) D$ still true, and find that this is related to the permutability of the ritting class $\mathcal{F}$.

\subsubsection{Lemma}

If $H \leqslant G, N 4 G$ and $H \perp G_{\pi}$, a Hall $\pi$-subsroup of $G$, then $\mathrm{HG}_{\pi} \cap \mathrm{N}=(\mathrm{H} \cap \mathrm{N})\left(G_{\pi} \cap N\right)$ so $(\mathrm{H} \cap \mathrm{I}) \perp\left(G_{\pi} \cap N\right)$.

\section{Proof}

Let $\mathrm{H}_{\pi^{\prime}}$ be a Hall $\pi^{\prime}$-subreroup of $\mathrm{H}$, then $\mathrm{H}_{\pi^{\prime}} G_{\pi}=H_{\pi}$ byanorderargument An element $g$ of $\mathrm{HG}_{\pi}$ is therofore uniquely expressible as $\varepsilon=\mathrm{xy}$ where $x \in \dot{H}_{\pi^{\prime}}$ and $y \in G_{\pi}$. If $x y \in \mathbb{N}$, then $x$ is a $\pi^{2}$-element of $N_{\pi}$, so $x \in N$ and hence also $y \in H$. Therefore $H G_{\pi} \cap N \leqslant$ $(H \cap H)\left(G_{\pi} \cap I\right)$. The converse is clear and the lemma proven. 
A.2.A T.

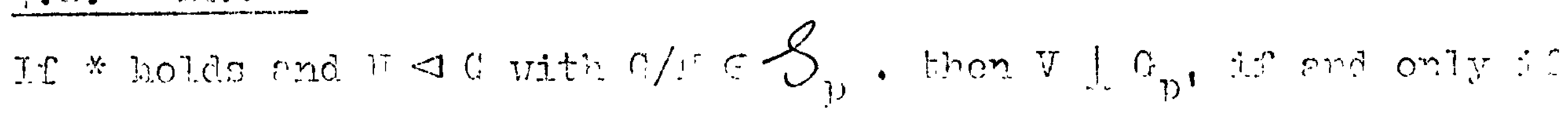

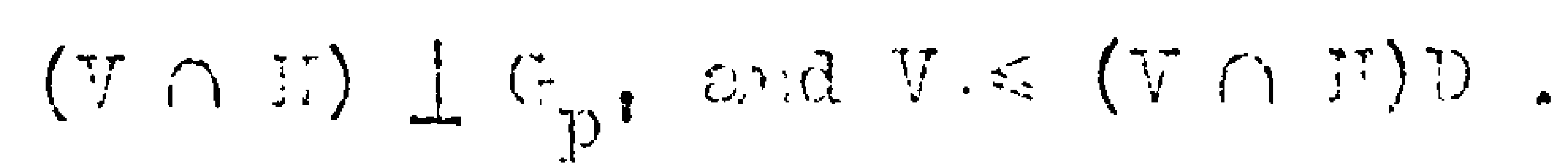

Droof

First suproso $V \perp\left(p\right.$, thon corteing $(V \cap N) \perp\left(G_{\eta}, \cap N\right)$ by 1.2 .3 and $\left(G_{p}, \cap N\right)=G_{p}$, since $G / I$ is 0 p-sroup. $V_{n}=V \cap G_{n}$

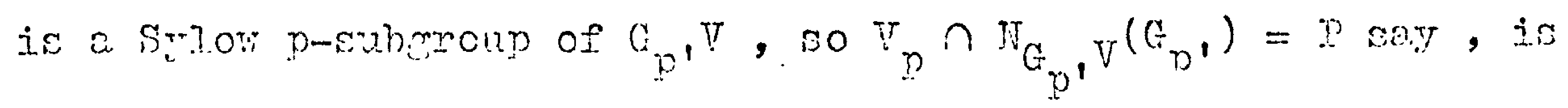
a Sylor p-Gubroup of $\mathrm{N}_{\mathrm{G}_{\mathrm{p}}, \mathrm{V}}\left(\mathrm{G}_{\mathrm{p}^{\prime}}\right)$. By the Frattini nreptent $\mathbb{G}_{G_{p}, V}\left(G_{p}\right)$ covers $G_{p}, V / G_{p}, V \cap N=G_{p}, V / G_{p},(V \cap N)($ by 4.2 .3$)$, which is a p-eroup, therefore $P$ covers this quotient too. So by the modular law

$$
\begin{aligned}
V & =V \cap(V \cap \mathbb{N}) C_{p^{\prime}}, P=(V \cap \mathbb{N})\left(V \cap G_{p^{\prime}} P\right)=(V \cap I)\left(V \cap G_{p^{\prime}}\right) P \\
& =(V \cap \mathbb{N}) P .
\end{aligned}
$$

Now $P=V_{p} \cap N_{G_{p}, V}\left(G_{p^{\prime}}\right) \leqslant G_{p} \cap N_{G}\left(G_{p^{\prime}}\right)=$ the unique Sylor p-subgroup of $D^{D}$. Thus $V \leqslant(V \cap N) D$.

Conversely if $V \leqslant(V \cap \mathbb{N}) D$ and $(V \cap N) \perp G_{p^{\prime}}$, then $V$ nomelimes $(V \cap \mathbb{N}) G_{p}$, since by 3.1.10 D normalizes both $V \cap \mathbb{N}$ and $G_{n}$. So $V(V \cap N) G_{p}$ is a subgroup of $G$, that is $V \perp G_{p}$.

\subsubsection{Iremma}

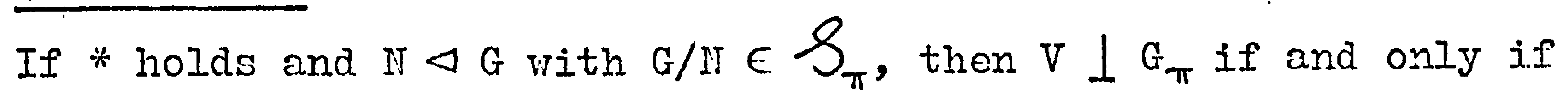
$(\mathrm{V} \cap \mathrm{NV}) \perp\left(\mathrm{G}_{\pi} \cap \mathrm{II}\right)$.

\section{Proof}

By 4.2 .3 the implication $\Rightarrow$ is clear. By 1.1 .7 a) $V \cap \mathrm{I}$ and $G_{\pi} \cap N$ are pronormal in $G$, so by $3.1 .6(V \cap N)\left(G_{\pi} \cap N\right)=T$ say, is pronormal in $G$. Therefore the Frattini arkudment implies that $\mathrm{I}_{G}(T)$ covers $G / N$, and by $3.1 .10 \Sigma \rightarrow \mathbb{N}_{G}(T)$. We deduce that $G_{\pi}$ normalizes $T$, so $(V \cap N) \perp G_{\pi}$. Now $\Sigma>V$, so $G_{\pi}$ contains a Hall $\pi$-suhcroup of $V$ and hence $V \leqslant(V \cap N) G_{\pi}$. It then follows that $V \perp G_{\pi}$. 
c) 4.2.4 ceanot be improved to the etetement :

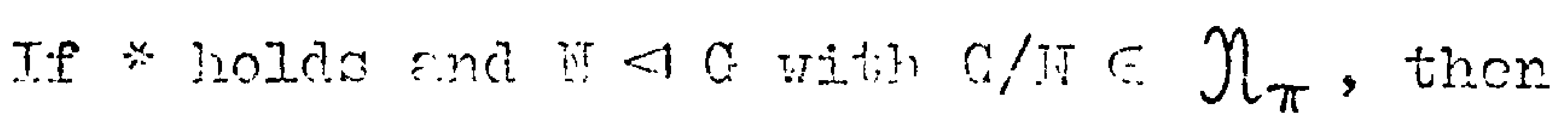

$V \perp G_{\pi^{\prime}} \Leftrightarrow(V \cap N) \perp G_{\pi^{\prime}}$ and $V \leqslant(V \cap \mathbb{N}) \mathrm{D}$.

The irplicetion $E$ certainly holds , the proof being as in the Iemro. Eovever, the exmple in $4.1 .3 \mathrm{c}$ ) shows thet $\Rightarrow$ is folsc. For let $\pi=19^{\prime}$ and let li be the nomed subcroup of $G$ of index 3 . $A$ s wo sar $\mathrm{H}=<\mathrm{a}>\mathrm{BE}$ is on $\mathcal{F}$-injector of $\mathrm{G}$ so $\mathrm{II} \cap \mathrm{IT}=\mathrm{BE}$. Obviously $H \perp G_{\pi^{\prime}}=G_{19}=E^{*}$. Wow $A=\left\langle a^{\prime}\right\rangle$ j.s clearly a system nornalizer of $\mathrm{J}$ (since $B, C$ and $D$ cover eccentric chief fectors) and thercfore $(H \cap I) D=\left\langle a^{\prime}\right\rangle B$ in this case. However $\mathrm{H} \neq<\mathrm{a}^{\prime}>\mathrm{BE}$ so $\mathrm{H} \neq(\mathrm{H} \cap \mathrm{N}) \mathrm{D}$.

b) It follows by the argupment used in the proof of $4.1 .2 \mathrm{~b}$ ) that if $T \leqslant G$ and $T \perp G_{\pi_{1}}, G_{\pi_{2}} \in \Sigma$, then $T \perp G_{\pi_{1}} \cap G_{\pi_{2}}$. Thus $T \perp G_{p}, E$ for all $p$ implios $T \perp \Sigma$.

\section{2 .7 THEOREM}

The following conditions on the Fitting class $\mathcal{F}$ are equivalent. a) $\mathcal{F}$ is permutable.

b) For any group $G$, implies $V \leqslant\left(V \cap G^{X}\right) D$.

c) For any group $G$, * implies $V \leqslant G_{\mathcal{F}} N_{G}\left(\Sigma \cap G_{F M n}\right)$. $\underline{P R O O F}$

a) $\Rightarrow$ b) : If * holds and $V \perp \Sigma$, then by $4.2 .4 V \leqslant\left(V \cap O^{p}(G)\right) D$ for all $p|| G \mid$. Therefore $D \cap V$ supplements, in $V$, each of its normal subsroups $V \cap O^{p}(G)$ for $p|| G \mid$. Now these have pairvise coprime indices in $\mathrm{V}$, so it follows that $\mathrm{D} \cap \mathrm{V}$ supplements their intersection $V \cap G^{\Re}$ and hence $V \leqslant\left(V \cap G^{\Re}\right) D$.

b) $\Rightarrow$ a) : Assuming b) holds we establish that $* \Rightarrow \perp \sum k y$ induction on $|G|$. So let $\mathbb{N}$ be a maximal normal subgroup of $G$ of index $\mathrm{p}$ say. $V \cap \mathrm{IJ}$ is the unique $\mathrm{F}$-injector of $\mathrm{N}$ into which the system $\Sigma \cap \mathbb{N}$ reduces, so by induction $V \cap \mathbb{N} \perp \Sigma \cap \mathbb{N}$. 


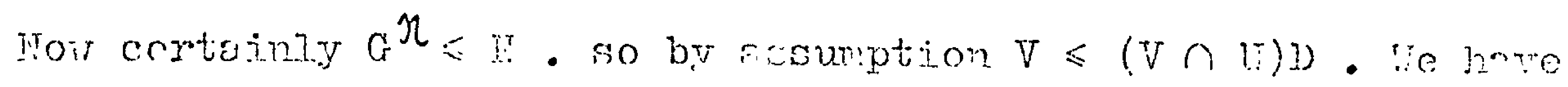

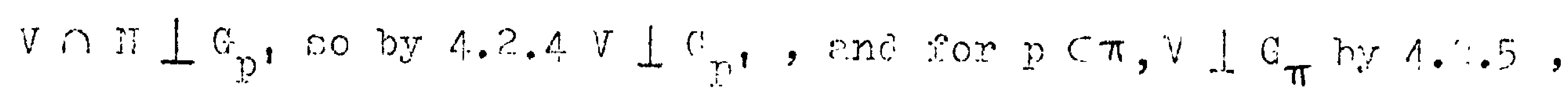
siving $\mathrm{V} \perp \Gamma$.

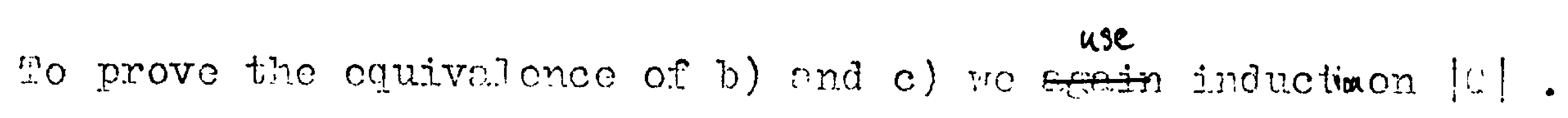

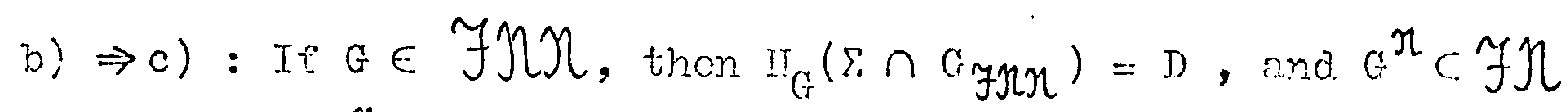
so that $V \cap G^{x} \leqslant G_{y}$. By assunption $V \leqslant\left(V \cap G^{x}\right) D$ thereforo $V \leqslant G_{y} N_{G}\left(S \cap G_{\mathcal{G}}(\Omega)\right.$, and $\left.c\right)$ holds in this cese.

We now assune $G \notin \mathcal{F} \backslash \eta$ and let $N$ be a meximal nomal subroup

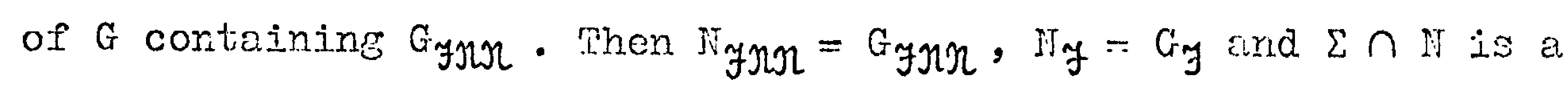
system of IT reducing into $V \cap N$ so by our induction hypothesis $V \cap N \leqslant G_{\mathcal{F}} N_{N}\left(\Sigma \cap G_{y n n}\right)$. Certainly $D \leqslant N_{G}\left(\Sigma \cap G_{F n n}\right)$ so $D$ normalizes $\mathbb{N}_{\mathrm{Ij}}\left(\Sigma \cap \mathrm{G}_{\text {gnn }}\right)$. We are assuming b) holds so $V \leqslant$ $\left(V \cap G^{n}\right) D$, therefore $V \leqslant(V \cap N) D \leqslant G_{y} J_{H}\left(\Sigma \cap G_{y n n}\right) D \leqslant$ $G_{y} I_{G}\left(\Sigma \cap G_{y n n}\right)$.

c) $\Rightarrow b):$ If $G \in \mathcal{F} \mathcal{G}$, then $G^{n} \in \mathcal{F}$ so $\left(V \cap G^{n}\right) D=G^{n} D=G$ and $b)$ holds trivially. When $G \notin \mathcal{F}, G_{\mathcal{F}} N_{G}\left(\Sigma \cap G_{\mathcal{F} n}\right) \neq c$. By Ho.II's celebrated results, $\Sigma>\mathbb{N}_{G}\left(\Sigma \cap G_{\text {yn }}\right)=\mathrm{I}$ sa.y, and furthermore $N_{T}(\Sigma \cap T)$, a system normelizer of $T$, is actually $D$. Let $D_{0}=N_{G_{F} T}\left(\Sigma \cap G_{\mathcal{F}} \mathrm{T}\right)$, then of course $D \leqslant D_{0}$. Homomorphism invorionce implies $D_{0} G_{f} / G_{f}$ is a system normalizer of $G_{F} T / G_{F} \cong$ $T / T \cap G_{F}$ of which $D\left(T \cap G_{F}\right) /\left(T \cap G_{F}\right)$ is a system normalizcr. Hence $D_{0} G_{f} / G_{f} \cong D\left(T \cap G_{f}\right) /\left(T \cap G_{f}\right) \cong D / D \cap\left(T \cap G_{f}\right)=D / D \cap G_{\mathcal{F}} \cong$ $D G_{\mathcal{F}} / G_{\mathcal{F}}$. Then $D \leqslant D_{0}$ forces $D_{0} G_{\mathcal{F}}=D G_{\mathcal{F}}$. c) holds so $V \leqslant G_{\mathcal{F}} \mathrm{i}^{\mathrm{i}}$, and wo are assumine that $G_{\mathcal{F}} \mathrm{T}<\mathrm{G}$; so the induction hypothesis gives $\dot{V} \leqslant\left(V \cap\left(G_{y} T\right)^{x}\right) D_{0}$. Wow clearly $\left(G_{y} T\right)^{x} \leqslant\left(G^{x} \cap G_{\mathcal{F}} T\right)$, and so $V \leqslant\left(V \cap G^{n} \cap G_{f^{i}}\right) D_{0}=\left(V \cap G^{n}\right) D_{0} \leqslant\left(V \cap G^{n}\right) D_{0} G_{y}=\left(V \cap G^{n}\right) D G_{\mathcal{F}}$. $G_{y} /\left(G^{n}\right)_{\mathcal{F}} \cong G_{f} G^{n / G}$, so D covers all the (central) chief factors of $G$ between $\left(G^{x}\right)_{y}$ and $G_{f}$, and therefore $G_{y} \leqslant\left(G^{x}\right)_{\mathcal{F}} D \leqslant\left(V \cap G^{x}\right) D$. With the previous inequity, this implies $V \leqslant\left(V \cap G^{x}\right) D$ and $\left.b\right)$ holds. 
5.1 Ytrons containment

In [18] cline introduces p partial orderine $\ll$ on the collection of saturated formations. If $\mathcal{E}$ and $\mathcal{F}$ are Fitting classes then analogously we say $\mathcal{E}$ is stronoly contained in 7 (rritten $\mathcal{E} \ll \mathcal{F}$ ) provided that, in each grop $G$, an $\mathcal{F}$-injector of $G$ contains some $E$-injector. In view of $1.1 .1 \mathrm{c})$ this is equivalent to the requirement that an $\xi$-jnjector or an $\mathcal{F}$-injector of a group $G$ is an $\xi$-injector of $G$. Clearly $\mathcal{F}$ implies $\mathcal{E} \subseteq \mathcal{F}$. This terminology applies nicely to the vork of previous chanters. For instance, $\mathcal{F}$ is a pormutehtee Fitting class $\Leftrightarrow \mathcal{F} \ll \mathcal{F}^{\pi}$ for all $\pi$, is obvious in the light of chapter 4 .

Cline attacks the problem of determining the saturates formations maximal. with respect to $\ll$, producing some which are (for example $\mathfrak{Y}^{2}$ ) and proving that certain others are not. Here wo take a proliminary look at the corresponding problom for Fitting classes and show that a strongly pronormal class, maximal in this sense, is necessarily normal.

\section{1 .1 Tsemma}

suppose $\mathcal{F}$ is a Fitting class such that $\mathcal{E}=0\left\{\mathrm{G} / \mathrm{G}_{\mathcal{F}}: G \in S\right\}$ $\neq \mathcal{H}$. Then $\mathcal{F}$ is normal.

\section{Proof}

Let $\mathrm{H}$ be an arbitrary group and $1 \neq K \in \mathcal{S}-\mathcal{X}$. Set $G=H \sim K$ and let $B$ be the base group of the wreath product.

Then $G_{y} \neq B$, otherwise $K$ is a quotient of $G / G_{f}$ and therefore e. member of $\mathcal{X}$, contrary to its choice. Let $G_{0}=B G_{y}, K_{0}=K \cap G_{0}$ and $r=\left|G: G_{0}\right|$. Then $G_{0}=B_{0} \cong H_{0} 2 K_{0}$ where $\mathrm{H}_{O}=\prod_{r} H$, the direct product of $r$ conies of $\mathrm{H}$, and $1 \neq \mathrm{K}_{0}$. 


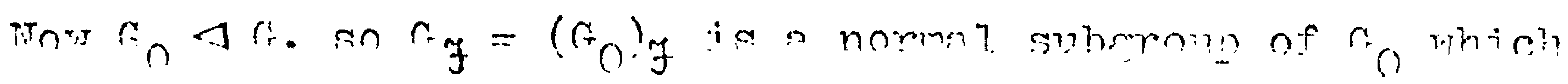

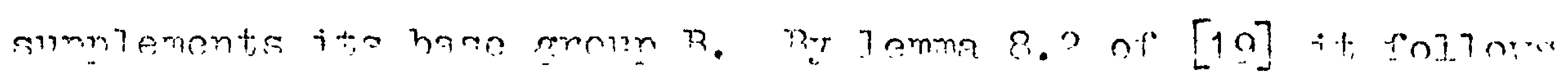

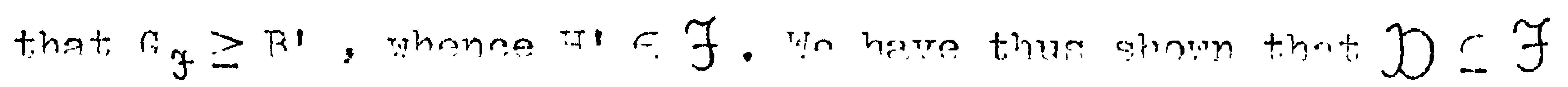
and an $\mathcal{F}$ is nomrnal.

$5.1 . ? \quad$ mFAnRFIn

Tf the rititing closs $\mathcal{F}(f \delta)$ is strongly nrounrmal and maxima?. with resnect to $\ll$, then $\mathcal{F}$ is nomml and inr each $G$, G/ty $F S_{p}$ for some fixed naine $n$.

THOnT

Pyr 4.1 .1 and our remark anove, the hynothesis imnlies $\mathcal{F} \ll \mathcal{F}$ for 971 primes a. Nor cleariy $\mathcal{Y}^{a}=8$ for a.1 a forces $\mathcal{F}=8$. so hr maximality there existir a nrime $n$ such that $\mathcal{f}=\mathcal{F}^{n}$, that is (by $2.6 .1 \mathrm{c})$ ) the $\mathcal{F}$-injectors of any $\mathrm{G}$ have n-nower index in $G$. In particular $p$ i.s unique. Ilet $V$ be an $\mathcal{F}$-injector of an arhitrery croun G. Ry hynothesis V strongo G and since $|G: V|$ is a porer of $p$, it follows that $\left\langle V_{p}{ }^{G}>\leqslant V\right.$ sn in fact, $V_{n} \leqslant r_{F}$ and therefore $V=r_{f} G_{p}$, for any $p$-comnlement $G_{p}$, of $G$ lying in $V$. Nor by $2.6 .5^{\prime}$ a) and the remark following j.t

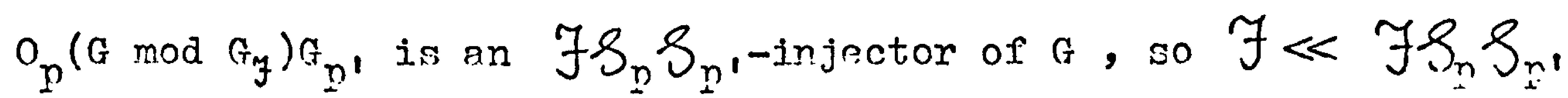
clearly $\mathcal{F}=\mathcal{F} \mathcal{S}_{\mathrm{p}} \mathcal{S}_{\mathrm{p}}$, implies $\mathcal{F}=\mathcal{S}$, so the other nossibility $F S_{n} S_{n^{\prime}}=S$, must hold. since $S_{n} S_{n^{\prime}}={ }^{\prime} S_{n} S_{n^{\prime}} \neq S$. Iemma 5.1 .1 gives us the desired conclusion.

Although re have seen that $\mathcal{F} \ll \mathcal{F}^{n}$ is not in ceneral true, we would be surprised if there were a Fitting class $\mathcal{F}$, maximal. with respect to $\ll$, which did not setisfy $\mathcal{F}=\mathcal{F}^{p}$ for some prime $\mathrm{n}$. Indeed ro suspect that the maximal classes are amons those described in the conclusion of 5.1 .2 , that is 1 normal classes of prime nower index ' Nor can we decide whether such classes are actually maximal. Clcarly $\ll$ and $\subseteq$ coincide for 


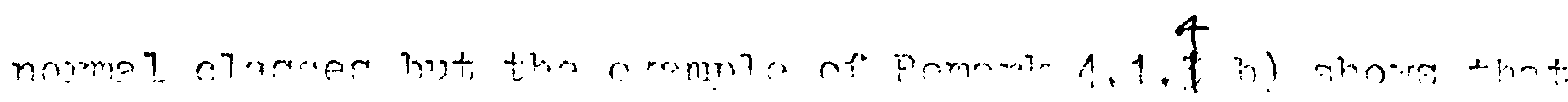

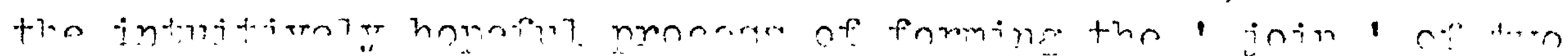

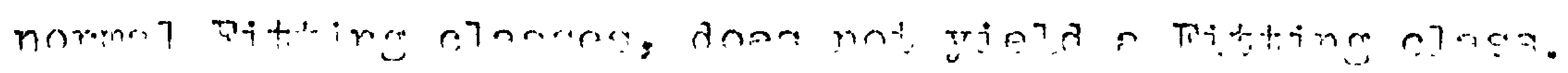

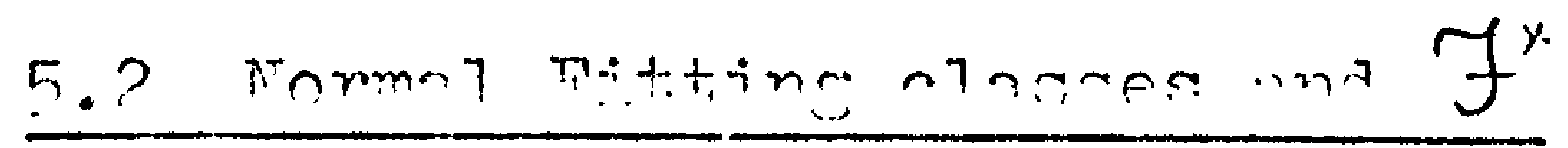

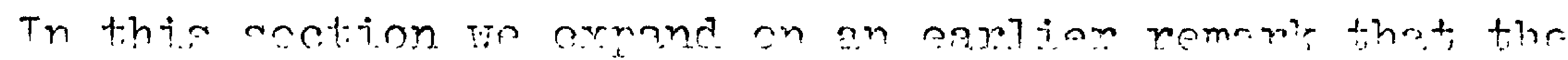

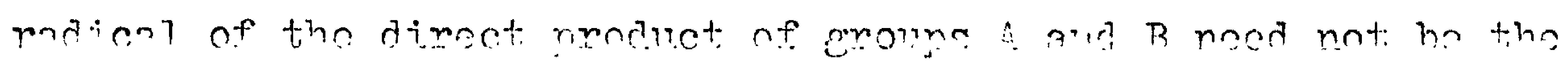

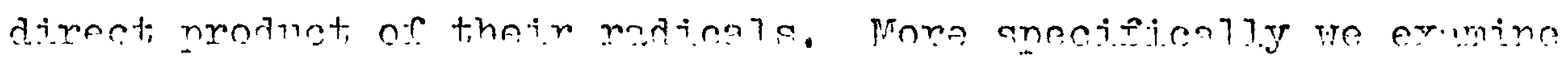

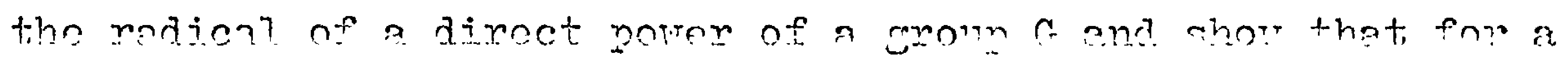

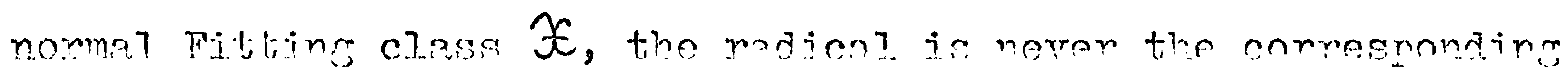

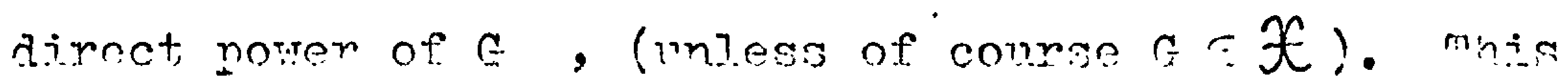
invertigation pits the samily of normel fitting clereses in a nner setting, and indeed wo shall. see that around each wittine closs $\mathcal{F}$ there is a snectrum of sitting classes (nartiy induced by the set of normal. classes) with pronerties close to thore nf $\mathcal{F}$.

Iet $\mathcal{F}$ be a Fitting class and ' $G$ a group. Iet $X=\prod_{n} G=$ $\left\{\left(\varepsilon_{1}, \varepsilon_{2}, \ldots, \varepsilon_{n}\right): \varepsilon_{i} \in q\right\}$ be the direct product of $n$ cories of $G$. Iet $G^{*}=\left\{g_{1}:\left(E_{1}, \varepsilon_{2}, \ldots, E_{n}\right) \in X_{F}\right\}$, the image of $X_{F}$ under the natural projection of $X$ onto its fjrst direct factor. Now $X_{F}$ char $X$ and, in the obvious way, $X$ admits both TAut $G$ and $\Sigma_{n}$ as sroups of automorphisms. It follows that ${ }^{n}{ }^{*}$ char $G$ and $G^{*}$ is the image of $X$ under the natural projection of $X$ onto any of its direct factors. Clearly $G_{y} \leqslant G^{*}$ and $\prod_{n} G_{f} \leqslant\left(\prod_{n} G_{\mathcal{F}} \leqslant \prod_{n} G^{*}\right.$.

\subsubsection{IJemma}

With the preceding notation

a) $G^{*} / G_{y}$ is Abelian

b) $\left(\prod_{n} G\right)_{j}=\left\{\left(\mathfrak{c}_{1}, \mathfrak{E}_{2}, \ldots, \varepsilon_{n}\right): E_{i} \in G^{*}\right.$.for all $i$ and $\left.\varepsilon_{1} \varepsilon_{2} \ldots \varepsilon_{n} \in G_{y}\right\}$ and $(G \times G)_{\mathcal{J}}=\left(G_{\mathcal{F}} \times G_{\mathfrak{F}}\right)<\left(g, g^{-1}\right): g \in G^{*}>$, so the definition of $G^{*}$ is independent of $n$. 


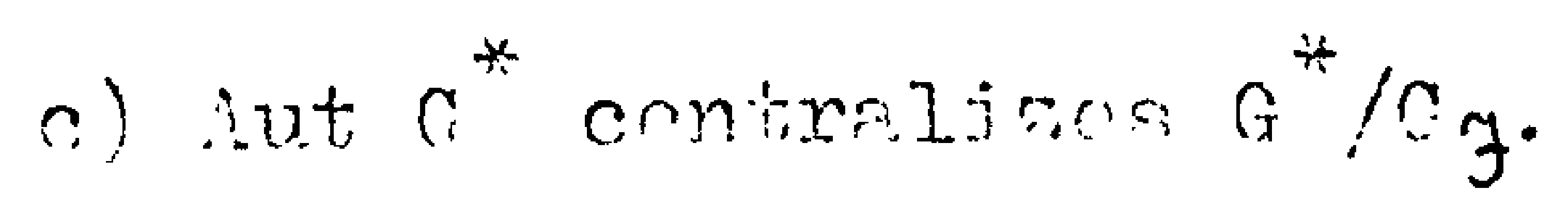

\section{Pronf}

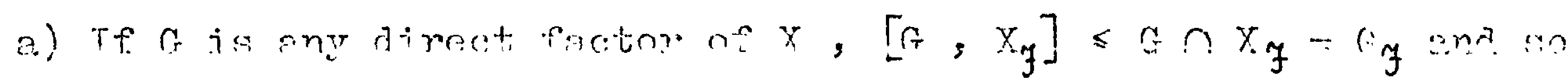

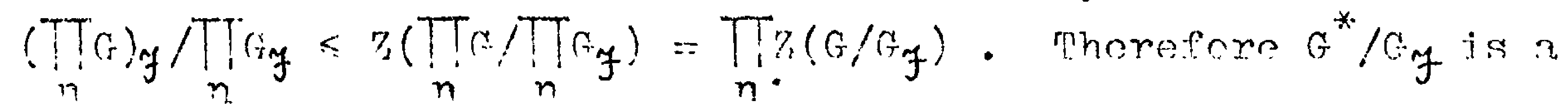
homomorphic imnge of an nbelinn erour, honce is itiself sbetion. b) Sunpose $\left(\varepsilon_{1}, s_{2}, \ldots, s_{n}\right) \in\left(\prod_{\eta} G\right)_{\mathcal{F}}$, then certain $y \varepsilon_{j} \varepsilon_{T}^{*}$ for each i hy the desinition of $G^{*}$. Aprothermore $\left(\varepsilon_{1}, \varepsilon_{2}, \ldots, \varepsilon_{n}\right) \in\left(\prod_{n} r\right)_{F}$

$$
\Rightarrow\left(s_{1}, g_{2}, \ldots, s_{n}, 1\right) \in\left(\prod_{n+1} r_{i}\right) \text { (bv conaidering the }
$$

obvinus emhedding $\prod_{n}\left(q \Delta \prod_{n}\left(r \times G=\prod_{n+1}^{n+1}(r)\right.\right.$

$$
\Rightarrow\left(1, \varepsilon_{p}, \ldots, \varepsilon_{n}, \varepsilon_{1}\right) \epsilon_{n+1}^{n+1}\left(\prod_{n} c\right)_{f} \text { (since } \underset{n+1}{\prod}()_{\mathcal{F}} \text { is }
$$

inveriant under the natimel antion of $\Gamma_{n+1}$ )

$$
\begin{aligned}
& \Rightarrow\left(\varepsilon_{1}, 1, \ldots, 1, \varepsilon_{1}^{-1}\right) \in\left(\prod_{n+1} G\right)_{7} \\
& \Rightarrow\left(\varepsilon_{1}, 1, \ldots, g_{1}^{-1}, 1\right) \in\left(\prod_{n+1}^{n+1}\right)_{F} \text { (action of } \Sigma_{n+1} \text { asosin) } \\
& \Rightarrow\left(\sigma_{1}, 1, \ldots, \sigma_{1}^{-1}\right) \subseteq\left(\prod_{n}^{n+1} G\right)_{f}\left(\prod_{n} G<\prod_{n+1} n\right. \text { agsin) }
\end{aligned}
$$

Similarly $\left(1, \ldots, g_{i}, ., g_{i}^{-1}\right) \in\left(\prod_{n} G\right)_{y}$ for $i=2, \ldots, n-1$, and so $\left(s_{1}, \varepsilon_{2}, \ldots, \varepsilon_{n-1}, s_{n-1}^{-1} \ldots \varepsilon_{2}^{-1} \varepsilon_{1}^{-1}\right) \in\left(\prod_{n} c_{f}\right)_{f}$, hence $\left.\left(1,1, \ldots,\left(g_{1} g_{2} \ldots g_{n}\right)^{-1}\right) \in i \prod_{n} G\right)_{F}$ which.imnlins $g_{1} e_{2} \ldots g_{n} \in G_{F}$.

Now if $\varepsilon_{i} \in G^{*}$, then $\left(\varepsilon_{1}, \ldots, \varepsilon_{i}, \ldots, \varepsilon_{n}\right) \in\left(\prod_{n} G_{i}\right.$ for suitable $g_{j} \in G^{*}$ $(j \neq i)$ by the definition of $G^{*}$. Then as above this implies $\left(1, \ldots, \varepsilon_{i} \ldots, g_{j}^{-1}, \in\left(\prod_{n} G_{q}\right.\right.$. So conversely if $\varepsilon_{1}, \ldots, g_{n} \in G^{*}$ and $g_{1} s_{2} \cdots g_{n} \in G_{\mathfrak{F}}$ we have

$$
\begin{aligned}
& \left(\xi_{1}, g_{2}, \ldots, g_{n-1}, g_{n-1}^{-1} \ldots . g_{2}^{-1} \varepsilon_{1}^{-1}\right) \in\left(\prod_{n} G\right)_{F} \\
& \text { and }\left(1,1, \ldots, \ldots, g_{1} \varepsilon_{2} \ldots \ldots \varepsilon_{n}\right) \in\left(\prod_{n} G\right)_{F}
\end{aligned}
$$

therefore $\left(g_{1}, \varepsilon_{2}, \ldots, \varepsilon_{n-1}, g_{n}\right) \in\left(\prod_{n} G\right)_{f}$

These manoeuvres shor $g \in G^{*} \Leftrightarrow\left(g, 1, \ldots, g^{-1}\right) \in\left(\prod G\right)_{7}$

$$
\Leftrightarrow\left(\varepsilon, g^{-1}\right) \in(G \times G)_{\mathcal{F}} \text {. }
$$

Joosely speaking $\left(\prod_{n} G\right)_{j} / \prod_{n} G_{f}$ is the subgroup of $\prod_{n} G^{*} / \prod_{n} G_{F}$ (rhich 


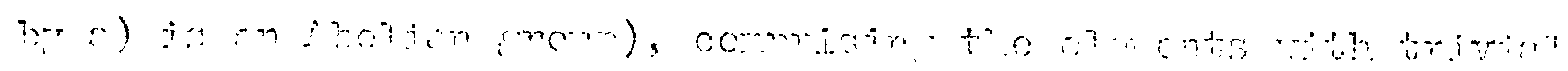

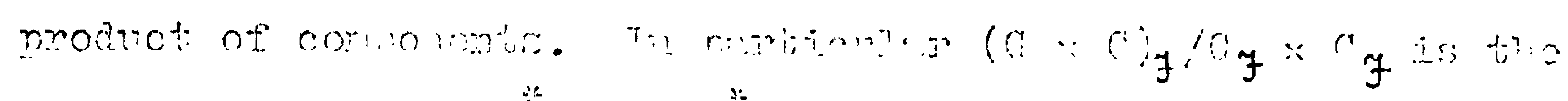

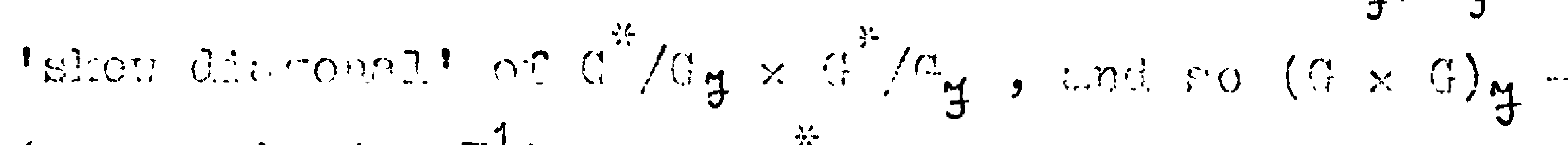

$\left(C_{y}: \sigma_{y}\right) \times\left(m, n^{-1}\right): r \in C^{*}>$.

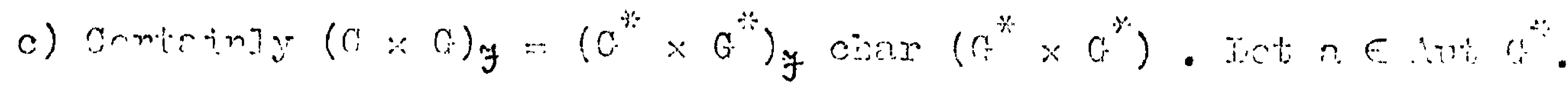

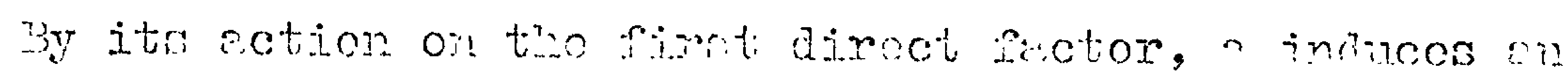

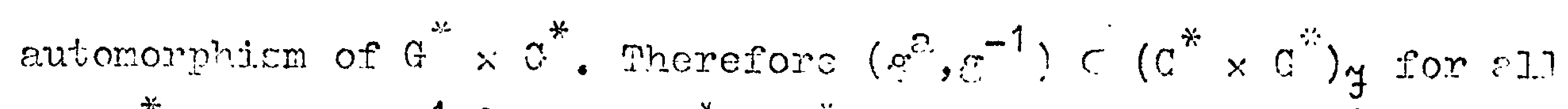

$\varepsilon \in G^{*}$. Hence $\left(\xi^{-1} \xi^{0}, 1\right) \subset\left(G^{*} \times G^{*}\right) y$, wich implies $\theta^{-1} \sigma^{2} \in C_{y}$

for :7.]. $s \in G^{*}$, thet is a cuntrelizos $G^{*} / G_{y}$.

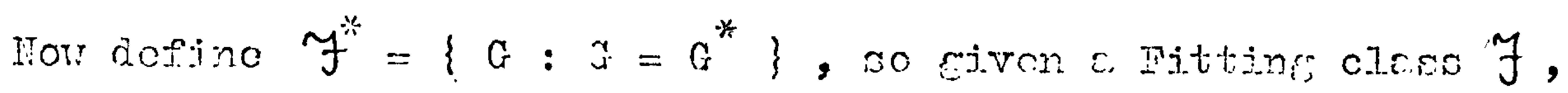
$\mathcal{F}^{*}-\left\{G:(G \times G)_{y}=(G y \times G y)<\left(c, G^{-1}\right): G \in G>\right\}$.

\section{2 .2 nITPORTIT:"}

Ii $\mathcal{F}$ is a Pitting cless then

e) $\mathcal{J}^{*}$ is a Fitting class

b). In the notation of Iemme $5.2 .1 \mathrm{G}^{*}=G_{\mathrm{F}^{*}}$

c) $\mathcal{F} \subseteq \mathcal{F}^{*}=\left(\mathcal{F}^{*}\right)^{*} \subseteq \mathcal{F} O \mathcal{Q}$ (the lest class is not recessarily Fitting of course)

a) $f$ is normal $\Leftrightarrow y^{*}=8$.

$\underline{\text { PPOOF" }}$

c) Suppose $N \triangleleft G \in \mathcal{F}^{*}$, then $\left.(G \times G)_{\mathcal{F}}=(G) \times G_{\mathcal{F}}\right)<\left(G, g^{-1}\right)$ : $\mathbb{G} \in G>$, therefore $(I \times N)_{\mathcal{F}}=(N \times N) \cap(G \times G)_{y} \geq\left(N_{F} \times N_{y}\right)<\left(n, n^{-1}\right): n \in N>$. Sc the image of $(\mathbb{N} \times \mathbb{N})_{\mathcal{F}}$ under the projection of $\mathbb{N} \times$ is onto $a$ direct factor is the whole of $N$, that is $N=N^{*}$ and $N \in \mathcal{F}^{*}$. Now suppose $\mathcal{f}^{*} \ni \mathrm{N}_{1}, \mathrm{~N}_{2} \triangleleft G=\mathrm{N}_{1} \mathrm{~N}_{2}$. For $i=1,2$, $G$ induces automorphisms of $\mathrm{N}_{i}$, so by $5.2 .1 \mathrm{c}$ ) and our supposition, $G$ centralizos $\mathrm{N}_{i} /\left(\mathrm{N}_{i}\right)_{\mathcal{F}}$, that is $\left[\mathrm{N}_{i}, G\right]_{\leqslant} \leqslant\left(\mathrm{N}_{j}\right)_{\mathcal{F}} \leqslant G_{\mathcal{j}}$. It follows that $G^{\prime}=\left[N_{1} H_{2}, G\right] \leqslant G G_{j}$. Now let $n_{1} n_{2}=g \in G$ where $n_{i} \in N_{j}$. Then $\left(\varepsilon, 8^{-1}\right)=\left(n_{1}, n_{2}, n_{2}^{-1} n_{1}^{-1}\right)=\left(n_{1}, n_{1}^{-1}\right)\left(n_{2}, n_{2}^{-1}\right)\left(1,\left[n_{2}^{-1}, n_{1}^{-1}\right]\right)$. Thus by supposition and our observation $\left(\varepsilon, E^{-1}\right) \in(G \times G) \mathcal{J}$. Projecting onto a direct factor gives $G=G^{*}$, so $G \in \mathcal{J}^{*}$. 


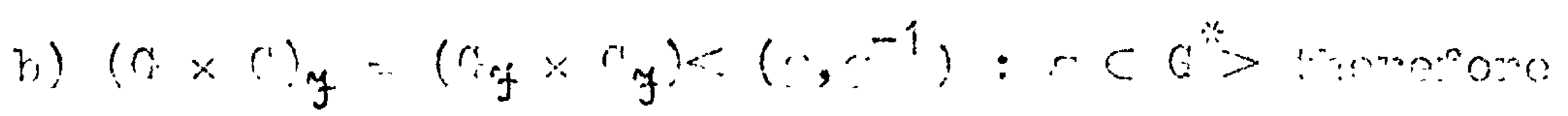

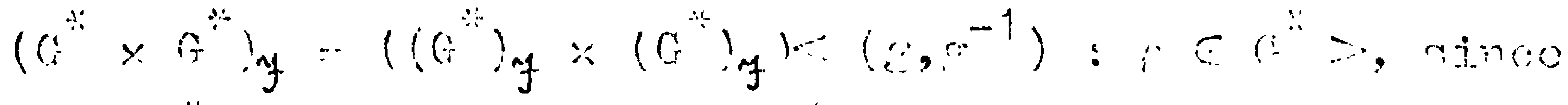

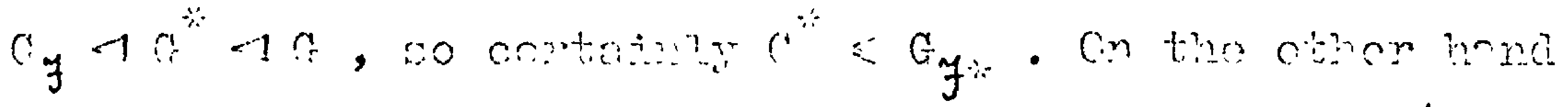

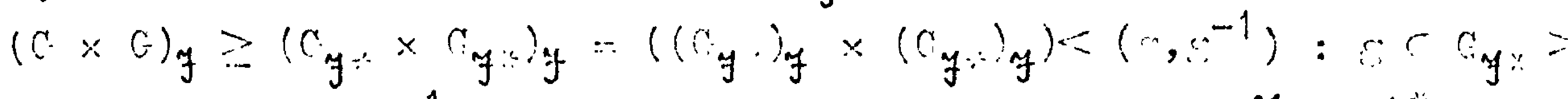

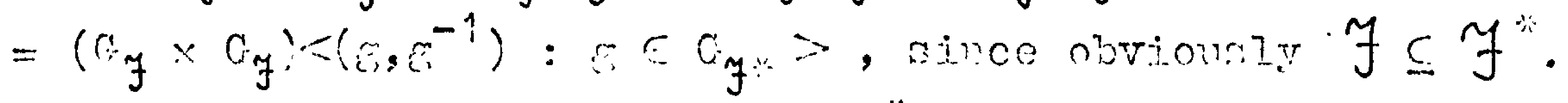

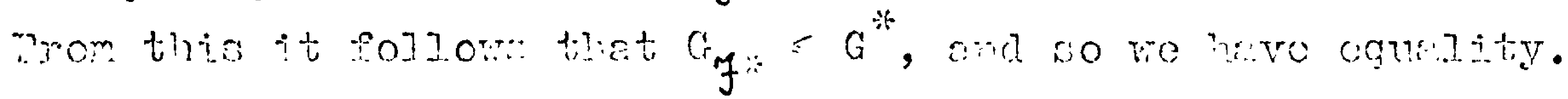

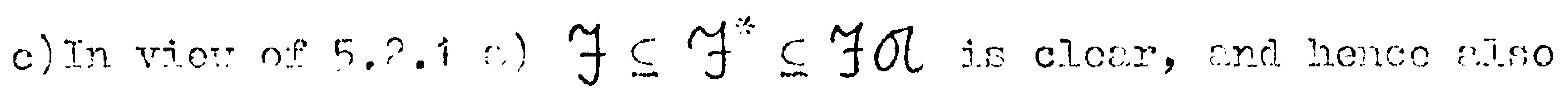
$\mathcal{F}^{*} \leq\left(\mathcal{F}^{*}\right)^{*}$, no it romeins to prove $\mathcal{F}^{*} \geq\left(\mathcal{F}^{*}\right)^{*}$. Sunnors $\Leftrightarrow \in\left(\mathcal{F}^{*}\right)^{*}$, then by refinition $(G \times C)_{\mathcal{F}^{*}}=$ $\left(G_{y *} \times G_{y *}\right)<\left(\varepsilon, e^{-1}\right): \varepsilon \in G>$, thenefore by $\left.b\right)$ $(G \times G \times G \times G)_{j}=\left((G \times G)_{\mathcal{F}} \times(G \times G)_{\mathcal{F}}\right)<\left(\varepsilon_{1}, \varepsilon_{2}, \varepsilon_{1}^{-1}, \varepsilon_{2}^{-1}\right):$

$$
\begin{aligned}
& \left(\tilde{E}_{1}, \tilde{E}_{2}\right) \subset\left(G \times G_{1}\right)_{y *}= \\
& \geq\left((G \times G)_{\mathcal{F}} \times(G \times G)_{\mathcal{F}}\right)<\left(\sigma, \sigma^{-1}, \varepsilon^{-1}, E\right): E \in G>\text {. }
\end{aligned}
$$

Thus $(c \times c \times G \times C)_{\mathcal{F}}$ projects onto ecch direct factor, sc $a \in \mathcal{F}^{*}$.

d) If $\mathcal{F}$ is a normal class then by 2.4 .3 G $\leqslant G_{y} \leqslant G$ for $0.11 \mathrm{G}$. In particular $\left(G 2 C_{2}\right)^{\prime} \leqslant\left(G 2 C_{2}\right)_{f}$. Ietting $C_{2}=\langle i>$ in the wroath product we have $[(\varepsilon, 1), i]=\left(\varepsilon^{-1}, \varepsilon\right)$, so $\left(\xi^{-1}, \varepsilon\right) \in(G \times G) \cap\left(G \sim C_{2}\right)_{y}=(G \times G)_{y}$. Therefore $G=G^{*}$, and so $\mathcal{F}^{*}=\mathcal{S}$. If $\mathcal{F}^{*}=S$, then by c) $\mathcal{F} a=\&$ so $\mathcal{F}$ is normal. $\square$

\section{2 .3 Remerks}

a) $\mathcal{F}=Q \mathcal{F} \rightarrow \mathcal{F}=\mathcal{F}^{*}$. For if $G \in \mathcal{F}^{*}$, then by decinition $G$ is a. homomorphic inase of $(G \times G)$ if , so $G \in \mathcal{F}$.

b) $\mathcal{F}=s \mathcal{F} \Rightarrow \mathcal{F}=\mathcal{F}^{*}$. For suppose $\mathcal{F} \subset \mathcal{F}^{*}$ and let $\mathrm{G}$ be of minimal order in $\mathcal{F}^{*}-\mathcal{F}$. Then $G$ has a unique moximal normal subgroup $I$ of index $\mathrm{p}$ say, and $N \in \mathcal{F}$. Furthermore by $5.2 .1 \mathrm{~b}$ ) and the definition of $\mathcal{F}^{*},\left(\prod_{p} G\right)_{\mathcal{F}}=\left\{\left(\varepsilon_{1}, \varepsilon_{2}, \ldots, \varepsilon_{p}\right): \varepsilon_{i} \in G\right.$ and $\left.\varepsilon_{1} \varepsilon_{2} \cdots \delta_{p} \in N\right\}$

which contains the diagonal $\{(\xi, \varepsilon, \ldots, \varepsilon): \xi \in G\}$, a subrroup isomorphic to $G$. So $G \in S \mathcal{F}=\mathcal{F}$ sivine a contrediction, and we acduce $\mathcal{F}=\mathcal{F}^{*}$. 


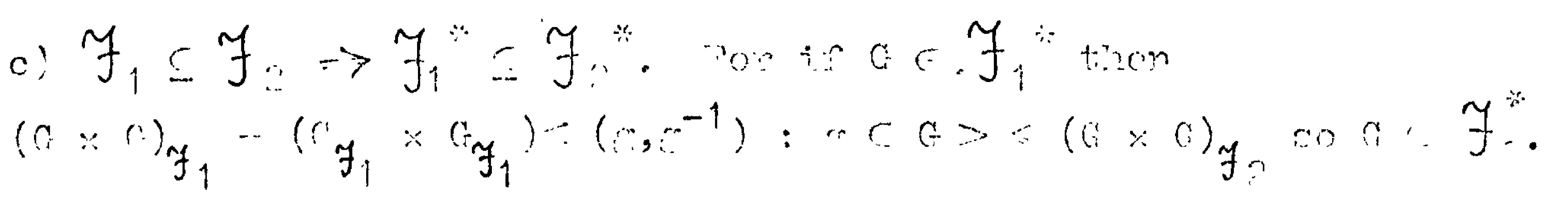

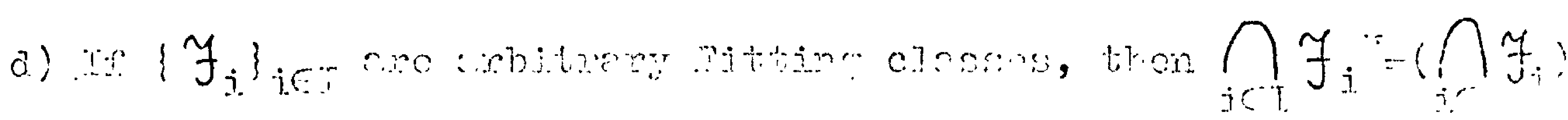

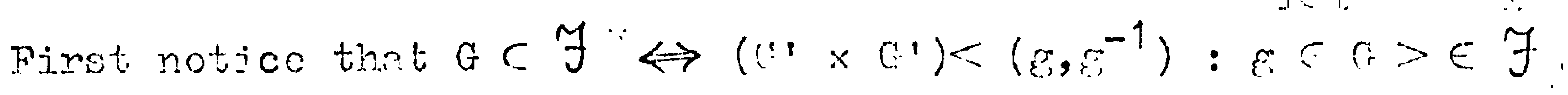

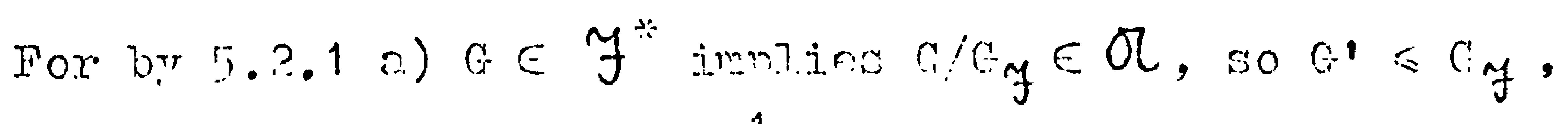
therciore $\left(a^{1} \times G^{1}\right)<\left(c, c^{-1}\right): \varepsilon \in G>\leqslant(c \times c)_{y}$ and $\left(G^{\prime} \times G^{\prime}\right)<\left(G, G^{-1}\right): \varepsilon \in G>\in \mathcal{F}$ forlons. Converscly this lest inclusion impries $G^{*}=G$ thet is $G \subset F^{*}$.

so $a, \in \bigcap_{i \in I} \mathcal{F}_{i}^{*} \Leftrightarrow\left(G^{\prime} \times C^{\prime}\right) \ll\left(s, s^{-1}\right): \varepsilon \in G>\in \bigcap_{i \in I} \mathcal{F}_{i} \Leftrightarrow G \in\left(\bigcap_{i \in I} \mathcal{F}_{i}\right)^{*}$. Given $\mathcal{F}$, derine $\mathcal{F}_{*}=\bigcap\left\{\mathcal{H}: \mathcal{H}^{*}=\mathcal{F}^{*}\right.$ \} so $\mathcal{F}_{*} \subseteq \mathcal{F} \subseteq \mathcal{F}^{*}$. e) $\mathcal{F}_{*} \subseteq \mathcal{X} \subseteq \mathcal{F}^{*}$ implics $\mathcal{K}^{*}=\mathcal{F}^{*}$ and $\mathcal{K}_{*}=\mathcal{F}_{*}$. For by d) $\left(\mathcal{F}_{* *}^{*}=\mathcal{F}^{*}\right.$, so by c) and 5.2.2 c) $\mathcal{F}^{*}=\left(\mathcal{F}_{*}^{*}\right)^{*} \subseteq \mathcal{K}^{*} \subseteq\left(\mathcal{F}^{*}\right)^{*}=\mathcal{F}^{*}$, Eivine $\mathcal{K}^{*}=\mathcal{F}^{*}$, so by definition $\mathcal{X}_{*}=\mathcal{F}_{*}$.

Hotice that by 5.2 .2 a) $\mathcal{S}_{*}=$ the unique smallest norm I rittine class.

\subsubsection{TIEORTII}

For each Fitting class $F$ and group in, $\mathcal{F} \ll \mathcal{F}^{*}$ and if $v^{*}$ is an

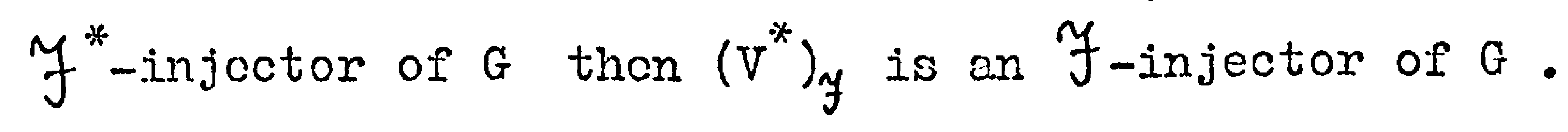

\section{PROOF :}

since $\mathcal{F} \subseteq \mathcal{F}^{*} \subseteq \mathcal{F} \Omega$, the second axsertjun follows at once from the first. Let $\mathrm{I}$ be a meximal normel suberoup of $G$ and $\Sigma$ a Sylow systen of $G$, and put $D=N_{G}(\Sigma)$. Let $V, V^{*}$ bo the unique $F_{\text {- and }}$ $\mathcal{F}^{*}$-injector rospectively of $G$ into which $\Sigma$ reduces. Then

$v=V \cap \mathbb{N}$ and $V^{*}=V^{*} \cap \mathbb{N}$ are the unique $\mathcal{F}$ - and $\mathcal{F}^{*}$-injectors of $N$ into which $\Sigma \cap \mathbb{N}$ reduces. We essume inductively thot for a group $H$ of order. Iess than $|G|$ with Sylov systen $\Omega$ :

the $\mathcal{F}$-injector of $\mathrm{H}$ into which $\Omega$ reduces $\leqslant$ the $\mathcal{F}^{*}$-injector of $H$ into which $\Omega$ reduces. 


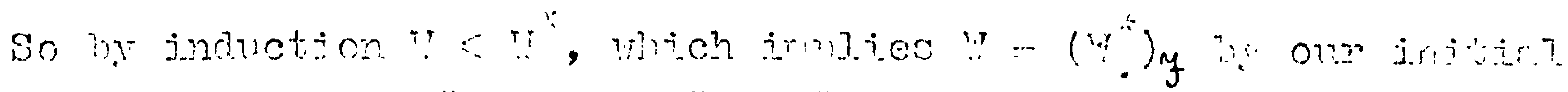

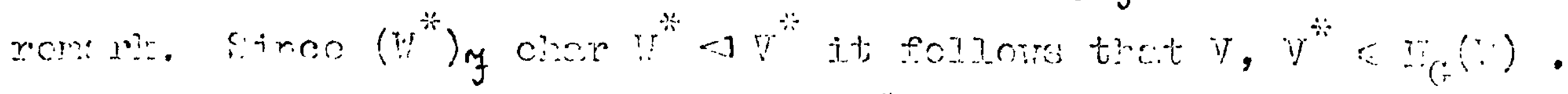

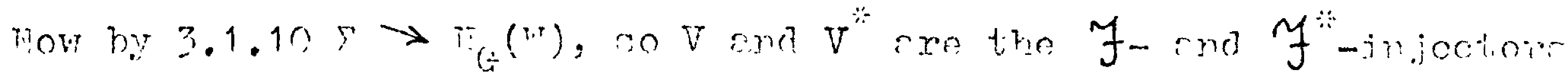

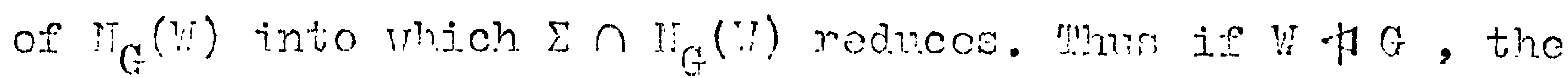
jnduction hypothosis implien $V \leqslant V^{*}$, so re moy assume $\because<{ }^{*}$.

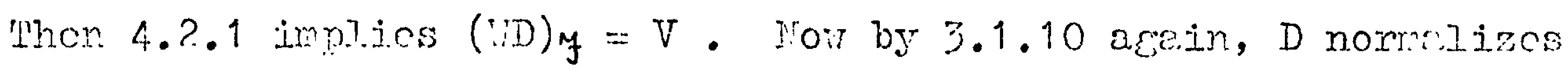
$V^{*}$, cond already $V \leqslant V^{*}$ so $V$ nommelizes $V^{*}$. By $3.1 .5 \mathrm{~g} \rightarrow V^{*}$, so ve nay assume $V V^{*}=G$. Thon $V^{*} \triangleleft G$ so $V^{*}=G_{f_{*}}$.

If $V \phi G$ then $Y=G_{y}$ since $\Gamma: V \mid=p$ in this casc. Ijy $5.2 .1 \mathrm{c}$ ) fut $G_{F_{*}}$ centralizes $G_{f_{*}} / G_{\mathcal{F}}$, so in perticular $G$ centralizes $V^{*} / i$, iut $G / V^{*}=V V^{*} / V^{*} \cong V / V^{*} \cap V$ is a quotient of $V / V \cong C_{p}$, so $\mathrm{G} / \mathrm{H}$ is 'central by cyclic', in particular, Abelian. Thereforo $V \triangleleft G$ after all, so certainly $\mathcal{F} \subseteq \mathcal{F}^{*}$ implies $V=G_{y} \leqslant G_{f^{*}}=V^{*}$.

5.2 .5 Corollary

$\mathcal{F}_{*} \subseteq \mathcal{H} \subseteq \mathcal{K} \subseteq \mathcal{Y}^{*}$ implies $\mathcal{H} \ll \mathcal{K}$, that is, for Fitting classes between $\mathcal{F}_{*}$ and $\mathcal{F}^{*}, \subseteq$ coincides with $\ll$.

proof

Put $\mathcal{F}=\mathcal{F}^{*}=\mathcal{H}^{*}=\mathcal{K}^{*}$ by $\left.5.2 .3 \mathrm{e}\right)$. Let $\mathrm{v}$ be ar $\mathcal{F}$-injector of the group G. Then 5.2.4 states that $V_{\mathcal{H}}$ and $V_{\mathcal{K}}$ a.re $\mathcal{H}-$ and $\mathcal{X}$-injectors of $\mathrm{g}$ respectively. Then $\mathcal{K} \subseteq \mathcal{K}$ implics $\nabla_{\mathcal{H}} \leqslant V_{\mathcal{X}}$, so $\mathcal{K}$ follows.

\section{2 .6 Coroljary}

If $\mathcal{F}$ is any Fitting class, $\mathcal{X}$ is a normal Fitting class and $V$ is an $\mathcal{F}$-injector of $G$ then $V_{X}$ is an $(\mathcal{F} \cap \mathcal{X})$-injector of $G$.

Proof

By 5.2 .3 a) and 5.2 .2 d) $(\mathcal{F} \cap \mathcal{X})^{*}=\mathcal{F}^{*} \cap \mathcal{X}^{*}=\mathcal{F}^{*}$.

so by 5.2 .2 e) and $5.2 .5 \mathcal{F} \cap \mathcal{X} \ll \mathcal{F} . V_{\mathfrak{X}} \triangleleft v$ therefore $v_{\mathfrak{X}} \in \mathcal{F}$ and hence is $(\mathcal{F} \cap \mathcal{X})$-maximal in $V$. It follows that $V_{\mathfrak{X}}$ is an $(\mathcal{F} \cap \mathcal{X})$-injector of $\mathrm{V}$ and we are done. 


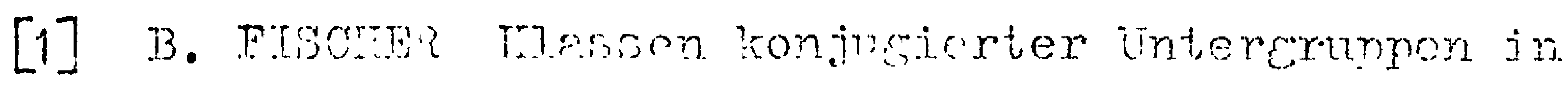
endlachen auflorbonen cruphen, Fabilitationsonrift Univ. Franlfurt (II) 1966

[2] B. FIsctor clessos of conjugate subcroups in finito solvable rouns, Yale University lecture notes 1966

[3] H. FITIUG Beitrëge zur Theorie der Gruppen endlicher Ordnung., Jahr. Deutsch. Prath. Ver. 481938

[4] B. HARTLEY On Fischer's dualization of Formation theory, P.I.M.S. 191969

[5] B. TISCHER, H. GASCHÜTZ and B. HARTLEY Injektoren endlicher auflôsbarer Gruppen, Nath. Zeit. 1021967

[6] II. SCHUNCK 5 -Untergruppen in endlichen auflösbaren Gruppen, Math. Zeit. 971967

[7] W. FASCHÜTZ Notes on soluble groups, A.N.U. Canberra Summer 1969-70

[8] D. BLESSEHOHL and H. GASCHÜTZ Úber normale schunckund Fittingklassen, Nath. Zeit. 1181970

[9] W. SUDBROCK Sylow Funktionen in endlichen Gruppen, Rend. Sem. Mat. Univ. Padova 361966

[10] G.A. CHAMBERS on the conjugacy of injectors, Proc. Am. Math. Soc. $28 \quad 7971$

[11] R. DARK A non-injective Fischer subgroup, To appear

[12] T.0. HAVKES On Fitting formations, Math. Zeit 117 1970 


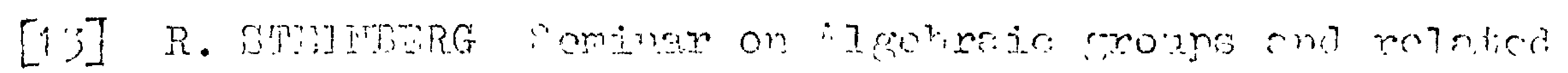

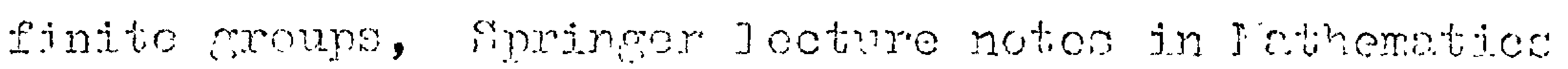
131

[14] G.A. CHLFBDRS p-nommaty embedded subgroups of finite soluble groups, Journal of MIgebra 161970

[15] $\Lambda$. MLIN A criterion for pronormality, J.J.T.S. 44 1969

[16] A. MAKAN Another characteristic conjugacy ciass of subgroups of finite soluble groups, Preprint A.H.U. Canberra, to appoar

[17] T.0. HAWKES An example in the theory of soluble groups Jroc. Camb. Phil. Soc. 67 1970

[18] E. CLTINE On an embedding property of generalized Carter subgroups, Pacific Journal of Iiaths. 291969

[19] P.H. NEUIANN on the structure of standard wreath products of groups, Math. Zeit. 841964. 